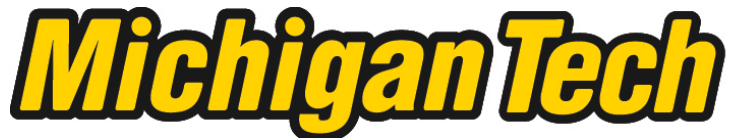 \\ Michigan Technological University Create the Future Digital Commons @ Michigan Tech
}

2010

\section{Three dimensional finite element simulation of polymer melting and flow in a single-screw extruder : optimization of screw channel geometry}

Atakan Altinkaynak

Michigan Technological University

Follow this and additional works at: https://digitalcommons.mtu.edu/etds

Part of the Mechanical Engineering Commons

Copyright 2010 Atakan Altinkaynak

Recommended Citation

Altinkaynak, Atakan, "Three dimensional finite element simulation of polymer melting and flow in a singlescrew extruder : optimization of screw channel geometry", Dissertation, Michigan Technological University, 2010.

https://doi.org/10.37099/mtu.dc.etds/346

Follow this and additional works at: https://digitalcommons.mtu.edu/etds

Part of the Mechanical Engineering Commons 
THREE DIMENSIONAL FINITE ELEMENT SIMULATION OF POLYMER MELTING AND FLOW IN A SINGLE-SCREW EXTRUDER: OPTIMIZATION OF SCREW CHANNEL GEOMETRY

By

ATAKAN ALTINKAYNAK

\author{
A DISSERTATION \\ Submitted in partial fulfillment of the requirements \\ for the degree of \\ DOCTOR OF PHILOSOPHY \\ (Mechanical Enginering-Engineering Mechanics) \\ MICHIGAN TECHNOLOGICAL UNIVERSITY \\ 2010
}

Copyright ( $)$ Atakan Altınkaynak 2010 
This dissertation, "Three Dimensional Finite Element Simulation of Polymer Melting and Flow in a Single-Screw Extruder: Optimization of Screw Channel Geometry", is hereby approved in partial fulfillment of the requirements for the degree of DOCTOR OF PHILOSOPHY in the field of Mechanical Enginering-Engineering Mechanics.

DEPARTMENT:

Mechanical Enginering-Engineering Mechanics

\section{Signatures:}

Dissertation Advisor

Dr. Mahesh Gupta

Committee

Dr. Fernando Ponta

Dr. Jeffrey Allen

Dr. Allan Struthers

Dr. Mark Spalding

Department Chair

Dr. William Predebon

Date 


\section{Contents}

List of Figures . . . . . . . . . . . . . . . . . . . . . . . . . xiii

List of Tables . . . . . . . . . . . . . . . . . . . . . . xiv

List of Algorithms . . . . . . . . . . . . . . . . . . . . . . . . $x v$

Statement of Co-Authorship . . . . . . . . . . . . . . . xvi

Acknowledgements ...................... . . xvii

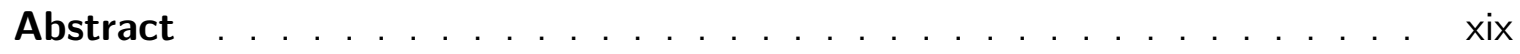

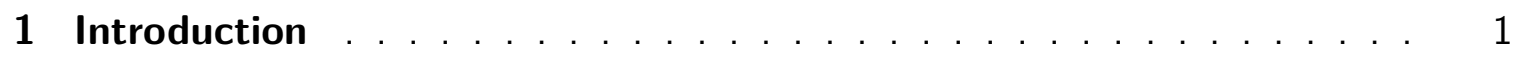

1.1 A Brief Introduction to Single-Screw Extruders . . . . . . . . . . . . 1

1.1.1 Previous Research on Polymer Melting in a Single-screw Extruder 3

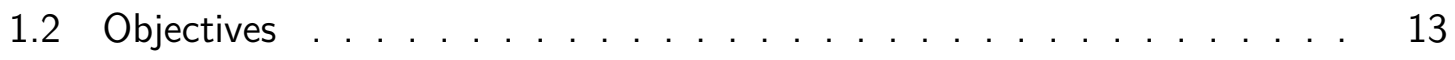

1.3 Thesis Organization . . . . . . . . . . . . . . . . . . . . . . 14

2 Experimental Methods, Results \& Discussion . . . . . . . . . . . . . . . 15

2.1 Maddock Screw-freezing Experiments . . . . . . . . . . . . . . . . . . 16

2.1.1 Experimental Results and Discussion . . . . . . . . . . . . . 18

2.2 Screw Simulator Experiments . . . . . . . . . . . . . . . . . . . . . . . 24

2.2 .1 Resins . . . . . . . . . . . . . . . . 26 
2.2.2 Calculation of Shear Stresses and Melting Fluxes . . . . . . . . . 27

2.2.3 Experimental Results and Discussion . . . . . . . . . . . . . . 29

2.3 Material Characterization Experiments . . . . . . . . . . . . . . 50

2.3.1 Experimental Results and Discussion . . . . . . . . . . . . 51

3 Numerical Methods, Results \& Discussion . . . . . . . . . . . . . . . . . 57

3.1 Governing Equations . . . . . . . . . . . . . . . . . . . . 58

3.1.1 Conservation of Mass (Continuity Equation) . . . . . . . . . . 58

3.1.2 Conservation of Momentum . . . . . . . . . . . . . . . . . . . . 59

3.1.3 Conservation of Energy . . . . . . . . . . . . . . . . . . . . 60

3.2 Finite Element Formulation . . . . . . . . . . . . . . . . . 61

3.2.1 Finite Element Formulation of Mass and Momentum Equations . 62

3.2.2 Finite Element Formulation of Energy Equation . . . . . . . . . . 64

3.3 Viscosity Model . . . . . . . . . . . . . . . . . . . 65

3.4 Numerical Simulations . . . . . . . . . . . . . . . . . . . . . . . . . 66

3.5 Use of Graphical Processing Unit for Finite Element Computations . . . . 68

3.6 Numerical Simulation Results . . . . . . . . . . . . . . . . . . . . . 72

3.6.1 ABS Resin Results: 6-8-7CR2.8 Screw . . . . . . . . . . . . . . 73

3.6.2 ABS Resin Results: Other Screws . . . . . . . . . . . . . . . . . 97

3.6.3 LDPE Resin Results . . . . . . . . . . . . . . . . . . . . . . . . 104

4 Optimization of a Screw Geometry in a Single-screw Extruder . . . . . 108

4.1 Formulation of the Optimization Problem . . . . . . . . . . . . . . . . . 111

4.2 Augmented Lagrangian Method: Extension to Inequality Constraints . . . 113

4.2.1 Extension to Inequality Constraint . . . . . . . . . . . . . . . . 114

4.3 Optimization Algorithm . . . . . . . . . . . . . . . . . . . 116

4.4 Screw Geometry Optimization . . . . . . . . . . . . . . . . . . . . 123 
5 Conclusions and Recommendations for Future Work . . . . . . . . . . . 136

5.1 Conclusions . . . . . . . . . . . . . . . . . . . . . . 136

5.2 Recommendations for Future Work . . . . . . . . . . . . . . . . . . . 137

References . . . . . . . . . . . . . . . . . . . . . . . . . . . 140

Appendix A Copyright Agreement with Wiley Periodicals, Inc. . . . . . . 152 


\section{List of Figures}

1.1 A schematic of a typical single-screw extruder. . . . . . . . . . . . 2

1.2 Melting mechanism at a cross section of an extruder channel (a) idealization, (b) photograph. . . . . . . . . . . . . . 4

2.1 Axial pressure profile of the ABS and LDPE resins with different screw configurations running at a screw speed of $60 \mathrm{rpm} \ldots \ldots \ldots 20$

2.2 Cross-sections of the solidified polymer obtained from the solidification experiments of the ABS resin using the 6-8-7CR2.0 screw. . . . . . . 21

2.3 Cross-sections of the solidified polymer obtained from the solidification experiments of the ABS resin using the 6-8-7CR2.4 screw. . . . . . . 21

2.4 Cross-sections of the solidified polymer obtained from the solidification experiments of the ABS resin using the 6-8-7CR2.8 screw. . . . . . . 22

2.5 Cross-sections of the solidified polymer obtained from the solidification experiments of the ABS resin using the 6-4-11CR2.8 screw. . . . . . 22

2.6 Cross-sections of the solidified polymer obtained from the solidification experiments of the ABS resin using the 6-8-7CR3.2 screw. . . . . . . 23

2.7 Cross-sections of the solidified polymer obtained from the solidification experiments of the LDPE resin using the 6-8-7CR2.8 screw. . . . . . 23

2.8 Experimental melting profiles of the ABS resin obtained from the Maddock screw-freezing experiments for different screw configurations. . . . . . . . . 24 
2.9 Schematic of the Screw Simulator.

2.10 Shear stress at the interface for the LDPE resin at $0.7 \mathrm{MPa}$ as a function of temperature and velocity.

2.11 Shear stress at the interface for the LLDPE resin at $0.7 \mathrm{MPa}$ as a function of temperature and velocity.

2.12 Shear stress at the interface for the ABS resin at $0.7 \mathrm{MPa}$ as a function of temperature and velocity.

2.13 Shear stress at the interface for the HIPS resin at $0.7 \mathrm{MPa}$ as a function of temperature and velocity.

2.14 Melting flux for the LDPE resin at $0.7 \mathrm{MPa}$ as a function of temperature and velocity.

2.15 Melting flux for the LLDPE resin at 0.7 MPa as a function of temperature and velocity.

2.16 Melting flux for the ABS resin at $0.7 \mathrm{MPa}$ as a function of temperature and velocity.

2.17 Melting flux for the HIPS resin at 0.7 MPa as a function of temperature

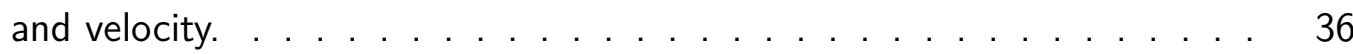

2.18 Melting flux for the four resins as a function of temperature at $61 \mathrm{~cm} / \mathrm{s} . \quad 37$

2.19 Shear stress at the interface for PC-3 resin at $0.7 \mathrm{MPa}$ as a function of velocity and temperature.

2.20 Shear stress at the interface for $\mathrm{PC}-6$ resin at $0.7 \mathrm{MPa}$ as a function of

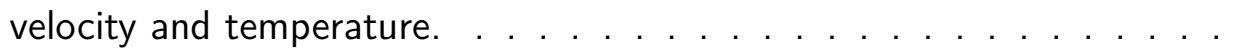

2.21 Shear stress at the interface for PC-22 resin at $0.7 \mathrm{MPa}$ as a function of velocity and temperature. . . . . . . . . . . . . . . . . . . 42

2.22 Shear stress as a function of velocity and MFR at $295{ }^{\circ} \mathrm{C}$. . . . . . . . . 
2.23 Melting flux for $\mathrm{PC}-3$ resin at $0.7 \mathrm{MPa}$ as a function of velocity and temperature.

2.24 Melting flux for PC-6 resin at $0.7 \mathrm{MPa}$ as a function of velocity and temperature.

2.25 Melting flux for $\mathrm{PC}-22$ resin at $0.7 \mathrm{MPa}$ as a function of velocity and temperature.

2.26 Melting flux as a function of velocity and MFR at $295{ }^{\circ} \mathrm{C} . \ldots . . . . . .46$

2.27 Schematic of the extruder used in the case study of PC-6 resin. . . . . . 47

2.28 Discharge pressure and motor current for an extruder running PC-6 resin with and without cooling water to the root of the screw. . . . . . . . . . 47

2.29 Discharge pressure and screw speed for an extruder running PC-6 resin with and without cooling water to the root of the screw. . . . . . . . . . 48

2.30 Shear viscosity data (symbols) and Cross-WLF model fit (curves) to the viscosity data for the $\mathrm{ABS}$ resin at 190,230 , and $270{ }^{\circ} \mathrm{C}$. . . . . . . . . 51

2.31 The change on zero-shear viscosity with respect to temperature for the ABS resin

2.32 Shear viscosity data (symbols) and Cross-WLF model fit (curves) to the viscosity data for the LDPE resin at 190,230 , and $240{ }^{\circ} \mathrm{C}$. . . . . . . .

2.33 Shear viscosity data (symbols) and Cross-WLF model fit (curves) to the viscosity data for the $\mathrm{PC}-3$ resin at $240,260,280$, and $300{ }^{\circ} \mathrm{C}$.

2.34 Shear viscosity data (symbols) and Cross-WLF model fit (curves) to the viscosity data for the PC- 6 resin at $240,260,280$, and $300{ }^{\circ} \mathrm{C}$. . . . .

2.35 Shear viscosity data (symbols) and Cross-WLF model fit (curves) to the viscosity data for the $\mathrm{PC}-22$ resin at $240,260,280$, and $300{ }^{\circ} \mathrm{C} . \ldots 54$

2.36 Shear viscosity for all $\mathrm{PC}$ resins at a temperature of $280{ }^{\circ} \mathrm{C}$. . . . . . . . 55 
3.1 (a) Unwound helical channel of a single-screw extruder with the barrel as an infinite plate moving on top (b) Finite element mesh. . . . . . . . .

3.2 Predicted temperature distribution in the melting section of the extruder for a constant screw temperature of $230{ }^{\circ} \mathrm{C}$ (left) and the corresponding experimental data from Fig. 2.4 (right). . . . . . . . . . . . . . . . . . 74

3.3 Comparison of experimental and numerical melting profile. . . . . . . . . 75

3.4 The predicted velocity profile corresponding to the melting profile shown in Fig. 3.2. . . . . . . . . . . . . . . . . . . . . . 77

3.5 Predicted pressure profile corresponding to the melting profile shown in Fig. 3.2. . . . . . . . . . . . . . . . . . 78

3.6 Predicted (a) polymer particle paths and (b) temperature of particle \#1 and \#2 in the melting section of the extruder.

3.7 Predicted temperature distribution in the melting section of the extruder for the finite element mesh (a) with two thin layers of elements (Fig. 3.2), and (b) with no thin layer of elements, at the barrel surface. . . . . . . . 82

3.8 Predicted average down-channel velocity of the solid-bed in the melting section of the extruder for different finite element mesh. . . . . . . . . .

3.9 Predicted temperature distribution in the melting section of the extruder for constant screw temperatures of (a) 230 , (b) 250 , and (c) $270{ }^{\circ} \mathrm{C}$. . .

3.10 Comparison of predicted melting profiles for screw temperatures of 230 , 250 , and $270{ }^{\circ} \mathrm{C} \ldots \ldots \ldots \ldots \ldots \ldots$

3.11 Predicted down-channel velocity distribution in the melting section of the extruder for constant screw temperatures of (a) 230, (b) 250, and (c) 270 ${ }^{\circ} \mathrm{C}$.

3.12 Predicted average down-channel velocity of the solid-bed in the melting section of the extruder for screw temperatures of 230,250 , and $270{ }^{\circ} \mathrm{C}$. . 
3.13 Average pressures recorded in experiment and corresponding predictions for different screw temperatures. . . . . . . . . . . . . . .

3.14 Predicted temperature distribution in the melting section of the extruder for constant screw temperatures of (a) 230 , (b) 250 , and (c) $270{ }^{\circ} \mathrm{C}$ (the zero-traction boundary condition enforced at the entrance of the screw

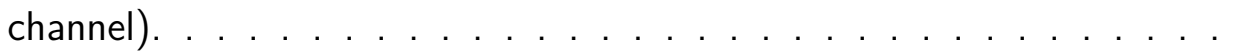

3.15 Predicted temperature distribution in the melting section of the extruder for a constant barrel temperature of (a) 230 , (b) 250 , and (c) $270{ }^{\circ} \mathrm{C}$. . .

3.16 Comparison of predicted melting profiles for barrel temperatures of 230 , 250 , and $270{ }^{\circ} \mathrm{C} \ldots \ldots \ldots \ldots \ldots \ldots \ldots$

3.17 Predicted temperature distribution in the melting section of the extruder for constant barrel temperatures of (a) 230 , (b) 250 , and (c) $270{ }^{\circ} \mathrm{C}$ (the zero-traction boundary condition enforced at the entrance of the screw channel). . . . . . . . . . . . . . . .

3.18 Average pressures recorded in experiment and corresponding predictions for different barrel temperatures.

3.19 Predicted temperature distribution in the melting section of the extruder when the shear viscosity at the same temperature and shear rate is; (a) reduced by half, (b) the same, and (c) doubled, compared to the basic flow case in Fig. 3.2. . . . . . . . . . . . . . . . . . . . . 96

3.20 Comparison of predicted melting profiles for different values of shear viscosity. 96

3.21 Average pressures recorded in the experiment and corresponding predictions for different shear viscosity values. . . . . . . . . . . . . .

3.22 Predicted temperature distribution in the melting section of the extruder for flow rates of (a) 0.75 times, (b) 1.00 times, and (c) 1.25 times the basic case flow rate. . . . . . . . . . . . . . . . . 
3.23 Comparison of predicted melting profiles for different values of flow rate.

3.24 Average pressures recorded in the experiment and corresponding predictions for different flow rate values. . . . . . . . . . . . . . . . . . . 99

3.25 Predicted temperature distribution for the 6-8-7CR2.0 screw (left) and the corresponding experimental data from Fig. 2.2 (right). . . . . . . . . 100

3.26 Predicted temperature distribution for the 6-8-7CR2.4 screw (left) and the corresponding experimental data from Fig. 2.3 (right). . . . . . . . . 100

3.27 Predicted temperature distribution for the 6-4-11CR2.8 screw (left) and the corresponding experimental data from Fig. 2.5 (right). . . . . . . . . 101

3.28 Predicted temperature distribution for the 6-8-7CR3.2 screw and the corresponding experimental data from Fig. 2.6 (right). . . . . . . . . . . 101

3.29 Comparison of experimental (filled circles) and numerical (lines) melting profile for 6-8-7CR2.0, 6-8-7CR2.4, and 6-8-7CR2.8 screws. . . . . . . 102

3.30 Comparison of experimental (filled circles) and numerical (lines) melting profile for 6-8-7CR2.8, 6-4-11CR2.8, and 6-8-7CR3.2 screws. . . . . . 102

3.31 Average pressures recorded in the experiments (filled circles) and corresponding predictions (lines) for different screw configurations. . . . . 103

3.32 Average pressures recorded in the experiments (filled circles) and corresponding predictions (lines) for different screw configurations. . . . . .

3.33 Predicted temperature distribution in the melting section of the extruder for the LDPE resin (left) and the corresponding experimental data from Fig. 2.7 (right). . . . . . . . . . . . . . . . . . . . . . . . 105

3.34 Comparison of experimental and numerical melting profile for the LDPE resin.

3.35 Average pressures recorded in the experiment (filled circles) and corresponding prediction (line) for the LDPE resin 
4.1 (a) Finite element mesh of tetrahedral elements representing the barrel geometry (b) Finite element mesh of triangular elements representing the surface of the screw. . . . . . . . . . . . . . . . . . . . . . . 110

4.2 The triangular screw mesh generated using the screw mesh generator code (a) Last 5 diameters of a screw (b) Axial view of the full screw and the discretized screw channel profile.

4.3 (a) Average pressure calculated at the entrance (b) average temperature calculated at the exit of the partitioned barrel mesh for the ABS resin (contour lines) and steps for the optimization algorithm (symbols with iteration numbers) . . . . . . . . . . . . . . . . . 126

4.4 (a) Average pressure calculated at the entrance (b) average temperature calculated at the exit of the partitioned barrel mesh for the PC-22 resin (contour lines) and steps for the optimization algorithm (symbols with iteration numbers). . . . . . . . . . . . . . . . . . . . . 127

4.5 (a) Average pressure calculated at the entrance (b) average temperature calculated at the exit of the partitioned barrel mesh for the LDPE resin (contour lines) and steps for the optimization algorithm (symbols with iteration numbers). . . . . . . . . . . . . . . . . . . . 128

4.6 Magnified results of the Figs. 4.3 and 4.5 for the (a) ABS and (b) LDPE resins, respectively.

4.7 (a) Average pressure calculated at the entrance (b) average temperature calculated at the exit of the partitioned barrel mesh for the LDPE resin at a flow rate of $60 \mathrm{~kg} / \mathrm{h}$ (contour lines) and steps for the optimization algorithm (symbols). 
4.8 (a) Average pressure calculated at the entrance (b) average temperature calculated at the exit of the partitioned barrel mesh for the LDPE resin at a barrel/screw temperature of $220^{\circ} \mathrm{C}$ (contour lines) and steps for the optimization algorithm (symbols) . . . . . . . . . . . . . . . . . . . 133

4.9 Steps of the optimization algorithm with a temperature constraint of 469.4 $\mathrm{K}$ (red symbols). The blue symbols from the optimization algorithm and the contour lines are the same as in Fig. 4.5 . . . . . . . . . . . . . . . . 134 


\section{List of Tables}

2.1 Various dimensions of the screws used in the screw-freezing experiments. 18

2.2 Experimental conditions obtained from the screw-freezing experiments. . 19

2.3 Flow properties and test conditions for the resins used in the Screw Simulator experiments. . . . . . . . . . . . . . . . . . . . . . . . . . 27

2.4 The values of the Cross-WLF viscosity model parameters for the ABS, LDPE, and PC resins. . . . . . . . . . . . . . . . . 55

2.5 Various material properties of the ABS, LDPE, and PC resins used in the numerical simulations. . . . . . . . . . . . . . . . . . . 56

4.1 Boundary conditions for the ABS, PC-22 and LDPE resins specified in the numerical simulations. . . . . . . . . . . . . . . . . . . . . . . 124 


\section{List of Algorithms}

4.1 Optimization code algorithm . . . . . . . . . . . . . . . . . . . 118

4.2 BFGS algorithm . . . . . . . . . . . . . . . . . . . . 121 


\section{Statement of Co-Authorship}

I would like to thank to my colleagues, Dr. Mahesh Gupta, Dr. Mark Spalding, and Sam Crabtree for their contribution to every stage of our works that were published in [1-4]. These publications were extensively used in the preparation of this dissertation. 


\section{Acknowledgements}

I would like to gratefully acknowledge my professors in my former university (Istanbul Technical University) for the knowledge that I received during my undergraduate and graduate (MSc.) years. Special thanks to Dr. Ahmet Aran for his encouragement about doing my Ph.D. studies in US.

I would like to give foremost thanks to my advisor Dr. Mahesh Gupta for giving me the opportunity to work on this research in the field of polymers and for his guidance and support during my Ph.D. Special thanks to Dr. Mark Spalding and Sam Crabtree at The Dow Chemical Company for their help and hospitality during my days in Midland. I also want to thank Mark and Sam for very useful discussions that we made on this research. I would like to thank The Dow Chemical Company for supporting this project and also giving me the opportunity to perform the experiments in their facilities.

The computer simulations presented in this paper were performed using the computational resources funded by the Center for Experimental Computing, Department of Mathematical Sciences at Michigan Technological University. These computational resources were crucial for my research and special thanks to Dr. Allan Struthers, Dave Kraus and Dr. Raymond Shaw for making these computational resources available for the Michigan Tech community. Other computational resources used in this research were TeraGrid resources provided by the Purdue University and a cluster provided by Dr. Phillip Merkey at Michigan Technological University for the MPI computations. 
I would like to thank to my committee members, Dr. Mark Spalding, Dr. Allan Struthers, Dr. Fernando Ponta, and Dr. Jeffrey Allen, for giving me valuable suggestions throughout my study and for reviewing my thesis. I would also like to thank to Dr. Mark Gockenbach and Dr. Kathleen Feigl for their valuable contributions.

Many thanks to the Mechanical Engineering Department for offering me the TA position and for the financial support. I would like to thank to my colleagues during my TA work, especially the lab coordinator Mike LaCourt, for making this assignment enjoyable and easy.

I would like to give many thanks to my friends from Michigan Tech, especially Rosa Flores and Efe Saran, for their friendship and for making this remote city a liveable and enjoyable place.

Last but not the least, I am grateful for my family and relatives for their endless encouragement and moral support. 


\section{Abstract}

Single-screw extrusion is one of the widely used processing methods in plastics industry, which was the third largest manufacturing industry in the United States in 2007 [5]. In order to optimize the single-screw extrusion process, tremendous efforts have been devoted for development of accurate models in the last fifty years, especially for polymer melting in screw extruders. This has led to a good qualitative understanding of the melting process; however, quantitative predictions of melting from various models often have a large error in comparison to the experimental data. Thus, even nowadays, process parameters and the geometry of the extruder channel for the single-screw extrusion are determined by trial and error. Since new polymers are developed frequently, finding the optimum parameters to extrude these polymers by trial and error is costly and time consuming. In order to reduce the time and experimental work required for optimizing the process parameters and the geometry of the extruder channel for a given polymer, the main goal of this research was to perform a coordinated experimental and numerical investigation of melting in screw extrusion.

In this work, a full three-dimensional finite element simulation of the two-phase flow in the melting and metering zones of a single-screw extruder was performed by solving the conservation equations for mass, momentum, and energy. The only attempt for such a three-dimensional simulation of melting in screw extruder was more than twenty years back. However, that work had only a limited success because of the capability of computers and mathematical algorithms available at that time. The dramatic improvement of 
computational power and mathematical knowledge now make it possible to run full 3-D simulations of two-phase flow in single-screw extruders on a desktop PC.

In order to verify the numerical predictions from the full 3-D simulations of two-phase flow in single-screw extruders, a detailed experimental study was performed. This experimental study included Maddock screw-freezing experiments, Screw Simulator experiments and material characterization experiments. Maddock screw-freezing experiments were performed in order to visualize the melting profile along the single-screw extruder channel with different screw geometry configurations. These melting profiles were compared with the simulation results. Screw Simulator experiments were performed to collect the shear stress and melting flux data for various polymers. Cone and plate viscometer experiments were performed to obtain the shear viscosity data which is needed in the simulations.

An optimization code was developed to optimize two screw geometry parameters, namely, screw lead (pitch) and depth in the metering section of a single-screw extruder, such that the output rate of the extruder was maximized without exceeding the maximum temperature value specified at the exit of the extruder. This optimization code used a mesh partitioning technique in order to obtain the flow domain. The simulations in this flow domain was performed using the code developed to simulate the two-phase flow in single-screw extruders. 


\section{Chapter 1}

\section{Introduction}

\subsection{A Brief Introduction to Single-Screw Extruders}

A single-screw extruder [6, 7] (Fig. 1.1) is one of the most commonly used devices in the industry to manufacture plastic parts. A single-screw extruder is used for melting a polymer and for generating the pressure required to pump the molten polymer through a die. Besides melting and pumping, polymer melt is also thoroughly mixed and homogenized in the extruder. Based upon experience over more than sixty years, the geometry of single-screw extruders has been continuously refined. Most single-screw extruders now have three distinct functional zones (Fig. 1.1).

$\triangleright$ The solid conveying (feed) section near the hopper has a large channel depth. The function of this section is to compact and convey the solid polymer along the helical screw channel.

$\triangleright$ In the compression (melting or transition) section, the channel depth decreases continuously. In this section, the heat generated by the mechanical work due to screw rotation and the heat conducted from the heated barrel melts the polymer.

$\triangleright$ The metering (melt conveying or pumping) section near the delivery end of the extruder has a constant shallow depth. This section is used to homogenize the polymer melt and to generate the pressure required to push the molten polymer through a die. 


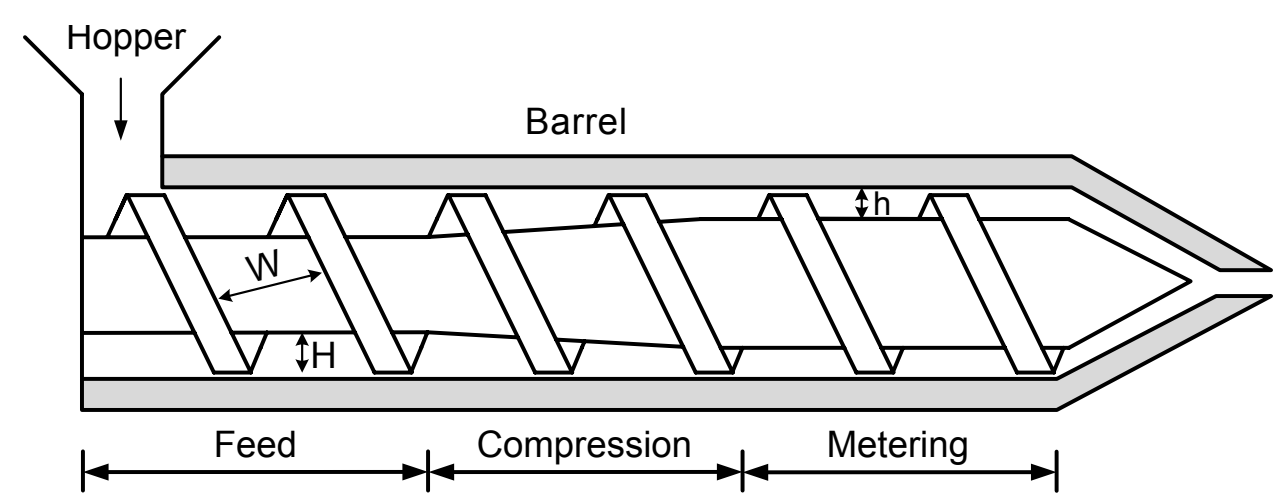

Figure 1.1: A schematic of a typical single-screw extruder.

It is desirable that a single-screw extruder has the highest throughput with good quality polymer melt at the extrudate. Higher output rates are possible with an increase in screw speed, however, as the screw speed is increased, a larger screw length is typically required to fully melt the polymer [8]. At higher screw speeds, melting can continue well beyond the compression section into the metering section. If the screw speed is increased beyond a limit, solid fragments can be discharged from the extruder, resulting in a poor quality extrudate. Output rate may be increased by increasing the size of the single-screw extruder. It was, however, shown by Chung [9] that as the size of single-screw extruder is increased, solid conveying and melt conveying rates scale up as the square of screw diameter, but the melting rate only scales up as a 1.75 power of screw diameter. Therefore, the performance of single-screw extruders is often limited by the melting rate and a good understanding of the melting in the extruder is the key for an optimum design and operation of a single-screw extruder.

As discussed in the next section, various publications in the literature provide a good understanding of the melting mechanism in single-screw extruders, and are useful in improving the design and control of the extrusion process. However, the melting process in single-screw extruders is still not fully understood because a large number of inter-dependent processing parameters influence the melting process. For instance, one 
of the most important parameters, the resin temperature, is not only related to the screw and barrel temperatures, but also depends on various temperature dependent material properties, such as viscosity, thermal conductivity and heat capacity, as well as depends on shear stress on the polymer-metal interface. A brief summary of the literature on experimental, analytical and numerical investigation of the melting process in single-screw extruders was presented in our earlier publication [1]. Details of the literature on this topic are given in the next section.

\subsubsection{Previous Research on Polymer Melting in a Single-screw Extruder}

During past five decades, numerous investigations have been performed on the topic of melting mechanism in plasticating extruders. Maddock [10] was the first one to obtain a significant understanding of melting in a single-screw extruder by performing screw freezing experiments. He observed that the first melt appears as a film of molten polymer between the hot barrel and compacted solid pellets (solid bed). Once the thickness of the melt film exceeds the clearance between the barrel and the screw flight, the polymer in the melt film is scraped by the active (pushing) flight of the screw to form a melt pool near the screw flight (Fig. 1.2). As the polymer in the melt pool increases, it exerts a considerable pressure on the solid bed causing it to deform continuously such that the width of the solid bed decreases along the channel, whereas the height of the solid bed essentially remains the same as the depth of the channel.

Further investigations based on screw freezing experiments of various polymers mostly confirmed the Maddock's melting mechanism [11-21]. However, some exceptions to the mechanism have been reported [22-25]. Menges and Klenk [22] noticed that for PVC the melt pool is formed on the passive flight and not on the active flight, which is in contrast 


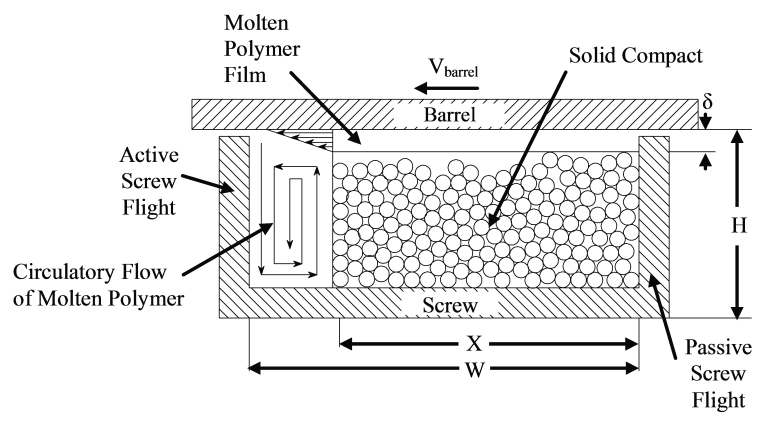

(a)

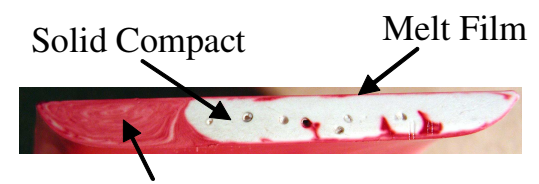

Melt Pool

Figure 1.2: Melting mechanism at a cross section of an extruder channel (a) idealization, (b) photograph.

to the results of Tadmor et al. [14] for PVC who found the melt pool on the active flight. On the other hand, Kulas and Thorshaug [19] found that for PVC the melt pool shifts from passive flight to active flight as the screw speed is increased. Dekker [24] observed that for polypropylene the solid bed is completely surrounded by polymer melt and did not notice melt pool on any side. For LDPE, Campbell et al. [25] also found melt all around the solid bed. They also observed that even though the solid-bed width decreased along the screw channel, it was the solid bed thickness which diminished to zero at the time of complete melting and not the bed width. Therefore, the Maddock melting mechanism may not be followed in all cases, particularly in the later stage of the melting.

Most of the detailed three-dimensional computational research in the literature on single-screw extruders has been limited to the metering section of the extruder. Moreover, because of the complexity of the two-phase flow in the compression (melting) section of the single-screw extruder, most of the research on melting of polymers is based upon a priori assumption. These simplified analysis do not solve the general conservation equations for mass, momentum, and energy over the complete flow domain and depending upon the validity of the assumed melting mechanism, good results were obtained by these 
simplified analysis, whereas erroneous values were predicted for various variables when the assumptions were violated. The melting models presented in the literature for single-screw extruders, in general, fall into one of the two groups: models with an assumed melting mechanism and models without an assumed melting mechanism. These models are briefly discussed in the next two sections.

\section{Models with an Assumed Melting Mechanism}

Based upon the experimental observations of Maddock and others discussed in the last section, a large number of publications in the literature [26-44] have attempted to model the melting of polymers in a single-screw extruder. All of these mechanistic models a priori assume the existence of a melting mechanism observed in a screw freezing experiment. Mass, momentum and energy balance equations are then developed for the different zones of the assumed melting mechanism. The main difference in the various mechanistic melting models in the literature is the assumed melting mechanism and simplifications employed in developing the mass, momentum and energy balance equations. All melting models also simplify the analysis by unwinding the helical screw channel into a straight channel and develop the equations in the reference frame fixed to the rotating screw; that is, the screw is considered stationary and barrel rotates in the opposite direction.

The first melting model for a single-screw extruder was developed by Tadmor [26] in 1966. Besides assuming that the Maddock melting mechanism holds for the complete melting section of an extruder, Tadmor also made the following additional simplifying assumptions.

$\triangleright$ Heat capacity and density in the solid and melt phases of the polymer are constant.

$\triangleright$ The downstream solid bed velocity is constant, which is determined from the mass flow rate of solid prior to the onset of melting. 
$\triangleright$ The lower melt film between the screw root and the solid bed, and between the passive screw flight and the solid bed, is ignored.

$\triangleright$ Thickness of the upper melt film between the barrel and the solid bed is constant.

$\triangleright$ The flow in the upper melt film is treated as a fully developed, isothermal, Newtonian drag flow.

$\triangleright$ For heat transfer analysis, the solid bed is treated as an infinite solid with temperature variations in thickness direction only.

$\triangleright$ Melting rate is constant across the solid bed width.

$\triangleright$ The polymer has a sharp melting point.

Based upon these simplifications, Tadmor obtained the Equation 1.1 for the solid bed profile by performing an energy and mass balance on the melt film between the solid bed and barrel.

$$
\frac{X}{W}=\left[1-\frac{z \sqrt{\frac{\left(C_{1} \rho_{m} V_{x}\right)}{2 \lambda W}}}{2 \rho_{s} V_{s z} H}\right]^{2}
$$

where

$$
C_{1}=\frac{\eta_{1} V_{r}^{2}}{2}+K_{m}\left(T_{b}-T_{m}\right)
$$

$X$ is the solid bed width, $W$ and $H$ are the width and height of screw channel, $\rho_{s}$ and $\rho_{m}$ are the densities of the solid and melt phases of the polymer, $\lambda$ is the heat of fusion of the polymer, $K_{m}$ and $\eta_{1}$ are the thermal conductivity and the constant viscosity of polymer melt, $z$ is the down channel direction, $V_{x}$ is the transverse velocity of barrel, $V_{s z}$ is the down-stream velocity of solid bed, $V_{r}$ is the velocity of barrel relative to solid bed, 
and $T_{b}$ and $T_{m}$ are, respectively, the barrel temperature and melting point of the polymer. Equation 1.1 can be used to determine the helical length of screw channel $\left(L_{m}\right)$ required for complete melting of the polymer (Equation 1.3).

$$
L_{m}=2 \rho_{s} V_{s z} H \sqrt{\frac{2 \lambda W}{C_{1} \rho_{m} V_{x}}}
$$

The Tadmor melting model is too simple for an accurate quantitative prediction of melting in a single-screw extruder for wide range of processing conditions and screw geometry. However, it does capture the qualitative trends in the melting process.

Since the work of Tadmor [26], a large number of modeling studies [27-44] have tried to improve the analysis by relaxing some of the assumptions in the original model. Tadmor et al. [14] upgraded the original model by including the dependence of viscosity on temperature and shear rate. Chung [27] considered the case with a finite solid bed depth. One of the limitations of the Tadmor model is that the solid bed width can increase beyond the width of the screw channel as the channel depth decreases in the compression zone of an extruder. To resolve this problem, Donovan $[29,30]$ relaxed the assumption of constant solid bed velocity and introduced a solid bed acceleration parameter, which allowed the solid bed velocity to increase in a prescribed manner. Instead of using a prescribed solid bed acceleration parameter, Edmondson and Fenner [33], Shapiro et al. [36], and Halmos et al. [37] attempted to calculate the solid bed velocity by a force balance on the solid bed and also allowed limited deformation of the solid bed. Vermeulen et al. [31, 32] and Shapiro et al. [36] analyzed the case with increasing melt film thickness in the cross-channel direction. Hinrichs and Lilleleht [28] used a helical coordinate system to include the effect of screw channel curvature and flight clearance on melting. Sundstrom and Young [45] found that the predicted melting rate is about $20 \%$ larger if convective heat transfer is included in the simulation. Most of the analysis listed above are for 
crystalline polymers which have a well defined melting point. In contrast, Sundstrom and Lo [38] analyzed melting of amorphous polymers with the solid-melt interface at the glass transition temperature of the polymer. Lindt $[34,40]$ developed the equations for the case when the solid bed is completely encapsulated from all four sides by a melt film. Shapiro, Pearson and coworkers $[36,37]$ further generalized such an analysis by developing a 5-zone model in which solid bed, melt pool, and the melt films on the barrel, screw root and passive screw flight, each are analyzed as separate zones, and the thickness of the melt film on barrel surface is also allowed to vary in the cross-channel direction.

Since some of the assumptions in the original Tadmor's model have been relaxed in the melting models discussed in the last paragraph, these models are expected to be more accurate. However, for these more complex models it is not possible to obtain a closed form analytical solution. Solutions for most of the complex melting models have been obtained numerically by using the finite difference method. Since most of the melting in various melting models is caused by the heat generated due to viscous dissipation in the melt film between the barrel and the solid bed, some authors in the literature developed the analytical solutions for the asymptotically limiting case of melting within a thin film of fluid between a hot moving surface and a melting solid. Vermeulen et al. [31] and Pearson [35] were among the first researchers to develop such analytical solutions. Mount et al. [42] generalized the asymptotic analysis for shear- and temperature-dependent viscosity. Chung and coworkers [46-50] also developed a Screw Simulator, which consists of a hot rotating drum. As the drum rotates a piece of solid molded polymer is forced against the drum surface at a controlled pressure. As the polymer melts, a thin film of polymer is formed on the drum surface, which is continuously scraped off the drum by a piece of softer metal. The Screw Simulator, which can be considered as an asymptotically limiting case of melting in a screw extruder, has been extensively used by Chung and coworkers [46-50] for experimental study of melting in screw extruders. However, the Screw Simulator and 
the analytical model of Vermeulen et al. [31], Pearson [35] and Mount et al. [42] only analyze the melting between barrel wall and solid bed. Potente [44] developed a more comprehensive analytical model which included the melting on the barrel surface as well as on the surfaces of the passive screw flight and screw root.

It is evident that tremendous effort has been devoted over the last forty years for the development of accurate models for melting in screw extruders. Depending upon the validity of the assumptions made, reasonable estimations of the melting rate and solid bed profile were also obtained for specific cases. However, large discrepancies were often observed between the experimental data and numerical predictions from various melting models [39, 49-54]. Mount and Chung [39] noticed that the solid bed profile predicted by Tadmor's model was in reasonable agreement with the experimental data. But, the melting rate predicted by the model had a large error. Mount and Chung [39] argued that the accurate prediction of the solid bed profile from Tadmor's model is only coincidental because many different solid bed profiles can be predicted for the same melting rate depending upon the method used to calculate the solid bed velocity. Therefore, a reasonable prediction of the solid bed profile is not a good indicator of the accuracy of a melting model. According to Mount and Chung [39], accurate prediction of the solid-bed profile from Tadmor's model results from the assumption of a constant solid bed velocity. This conclusion of Mount and Chung may be true because it was noticed by Lindt and Elbirli [52] that prediction from the simple Tadmor's model are often more accurate than from other more complex models which are based upon more rigorous analysis of solid bed velocity. However, because of the assumption of a constant solid bed velocity, Tadmor's model predicts unreasonably large solid bed width (larger than channel width) for higher throughputs in tapered channels. Cox and Fenner [51] obtained experimental data for the melting of three different thermoplastics in three different screw designs. By comparing their experimental data with predictions from their melting model, which calculated the 
solid bed velocity from a force balance, they concluded that predictions from melting models with a priori assumption of melting mechanism agree with the experimental data only in early stages of melting and agreement deteriorates in later stages. Mount [54] also noticed that various melting models in general over predict the melting rate for amorphous polymers.

Besides these discrepancies between the experimental data and predictions from the melting models, some of the more recent publications show that even some of the qualitative trends predicted by various melting models are contradictory to experimental observations. As expected, all melting models predict higher melting rates for polymers with higher viscosity because most of the melting $(80-90 \%)$ is caused by the heat generated due to viscous dissipations in the melt film between the barrel and the solid bed [7]. However, Hong et al. [49] did not observe this expected trend in their experimental work with various grades of polystyrenes and LDPE. Hong et al. [49] noticed that melting rate was lower for polystyrenes with higher viscosity, whereas for LDPE melting rate had no clear trend (decreased, increased and again decreased) as the viscosity of the polymer melt was increased. A similar unexpected trend was observed by Hogan et al. [50] for polycarbonate. Their experimental data also showed that the melting rate was lower for the grade of polycarbonate with the higher viscosity. Hong et al. [49] and Hogan et al. [50] concluded that the melting rate not only depends upon the rate of heat generation but also on material removal rate, and that their seemingly contradictory results indicate the complex nature of the melting process in screw extruders.

Limitations of the melting models with a priori assumption are also evident from the experimental work of Stangland et al. [53] with polypropylene. Stangland et al. [53] compared the melting of polypropylene with LLDPE. Even though polypropylene has better physical properties than LLDPE for many applications, it is much more difficult to melt. Since the viscosity and thermal conductivity of the LLDPE and polypropylene used 
were similar and polypropylene actually had lower heat capacity and heat of fusion than LLDPE, polypropylene was expected to melt faster. But, Stangland et al. [53] noticed that the melting rate for polypropylene was as much as 30\% lower than that for LLDPE.

Even though Tadmor-type melting models have been very helpful in elucidating the qualitative trends in melting of polymers in single-screw extruders, the melting process is too complex for a single melting mechanism to be valid for the complete melting section of all extruders for all polymers. Instead of a priori assuming the existence of a melting mechanism, melting of a polymer in single-screw extruders can be simulated by solving the conservation equation for mass, momentum and energy along with a constitutive equation for the polymer being used. Some of the past attempts for such a general simulation of the flow in extruders are discussed in the next section.

\section{Simulation of Melting Without an Assumed Mechanism}

With the tremendous advancement in the speed of computers over the last decade, there is no reason at this point to limit the melting simulation to an assumed mechanism. Instead, melting can be accurately simulated by solving the conservation equations for mass, momentum and energy along with the constitutive equation for a polymer. There have been a few attempts in the literature for such a detailed simulation of polymer melting in a screw extruder. Syrjala [55] performed such an analysis of the melting process. However, by assuming a fully developed flow in the down-channel direction, Syrjala [55] only performed a two-dimensional marching-type analysis in a cross section of the unwound screw channel. In this approach, the velocity and pressure at various cross-sections of the extruder channel are determined by marching along the channel. Even though, Syrjala [55] did not assume a melting mechanism, one of the main simplifying assumptions in the Tadmor model, namely, a constant solid bed velocity which is determined from the mass flow rate of the compacted solid at the beginning of the melting section, was 
used in Syrjala's work. Also, the Syrjala [55] approach cannot be used for a tapered channel because the assumption of fully developed down-channel velocity is no longer valid. Syrjala's results [55] conform to the Maddock melting mechanism, however, because of the simplifying assumptions, he obtained a physically unrealistic pressure rise in the initial stage of melting. In an earlier publication [56], Syrjala used the same approach as in reference [55], but applied it to the melt flow in the metering section of an extruder. In this earlier publication on the flow in metering section, predictions from the two-dimensional marching-type solution and a full three-dimensional simulation were compared. The velocity, pressure and temperature predicted by the two different approaches were found to have large differences. Therefore, Syrjala's two-dimensional marching-type simulation of melting in a screw extruder is a step in the right direction. However, for a complete understanding of various complexities in the melting process a full three-dimensional simulation of the two-phase flow in the melting region of the extruder is needed.

A full three-dimensional simulation of melting in a single-screw extruder was attempted by Viriyayuthakorn and Kassahun [57] more than twenty years ago. However, no further progress has been reported since then. The pictures of the predicted solid bed profile in this paper are not very clear but it appears that at the beginning of the compression section, the solid bed is completely encapsulated by polymer melt. Viriyayuthakorn and Kassahun employed the standard Galerkin formulation [58] not only for the momentum and mass balance equations but also for the energy equation. Even though it was not as well documented at the time of the publication by Viriyayuthakorn and Kassahun, it is well known now that for stability of the simulation and to be able to accurately capture the heat convection term in the energy balance equation, an upwind scheme [59-65] must be used in the finite element formulation of the energy equation. This lack of an upwind scheme, and the corresponding inability to accurately capture the convection 
term in the simulation, is probably the reason why Viriyayuthakorn and Kassahun could not capture the melt pool on the active flight of the screw. Besides this inaccuracy in the numerical scheme, the program needed a very large computational capability. In light of the limitations on the algorithms and computer technology available at the time, the work of Viriyayuthakorn and Kassahun [57] was a bold step in the right direction, but only a limited success was achieved because of the limitations discussed above.

Another reason for the limited impact of the two attempts for melting simulation without an assumed melting mechanism $[56,57]$ is the lack of experimental verification in both of these publications. Without any experimental verification it is difficult to judge the accuracy of the numerical predictions.

\subsection{Objectives}

Even though the extensive research on melting in screw extruders has been very successful in elucidating the general trends in the melting process, the recent experimental data $[25,49,50,53]$ contradicts many of the traditional beliefs and understandings on melting in screw extruders. These recent results clearly show the complex nature of melting in screw extruders and the inadequacy of knowledge we have on this topic. Therefore, the objective of this research is to perform a coordinated experimental and numerical investigation of melting in screw extrusion for a thorough understanding of various complexities in the melting process. Towards this goal, specific tasks which were completed during the course of this research are listed below.

$\triangleright$ Perform a full three-dimensional finite element simulation of the two-phase flow in the melting and metering zones of a single-screw extruder.

$\triangleright$ Use of a graphic processing unit (GPU) in order to reduce the computation time. 
$\triangleright$ Accurately simulate the material behavior of different polymers.

$\triangleright$ Verify the numerical predictions by performing a detailed experimental study of melting in a single-screw extruder.

$\triangleright$ Develop software for the optimization of a screw channel geometry such that the fastest possible output rate is achieved for a given polymer.

\subsection{Thesis Organization}

In the first chapter, along with a brief introduction to single-screw extruders, a detailed summary of the previous research on the topic of polymer melting in single-screw extruders was given. The rest of this dissertation is organized in the following manner. The experimental methods used in the investigation of the polymer melting and flow in single-screw extruders are described in Chapter 2. The experimental results along with the discussion is also presented in Chapter 2. In Chapter 3, an overview of the equations used to characterize the flow of polymers and the implementation of the finite element method to these equations are discussed. The details of the numerical simulations followed by the numerical results and discussion are also given in Chapter 3. The theory used and the code developed for the optimization of a screw geometry in single-screw extruders is described in Chapter 4. The dissertation ends with Chapter 5, in which the conclusions from this work is summarized and the recommendations for future work is given. 


\section{Chapter 2}

\section{Investigation of Polymer Melting and}

\section{Flow in Single-screw Extruders:}

\section{Experimental Methods, Results and}

\section{Discussion}

In order to investigate the polymer melting and flow in single-screw extruders and to verify the numerical predictions from the full 3-D finite element simulations of two-phase flow, a detailed experimental study was performed at The Dow Chemical Company. This experimental study included Maddock screw-freezing experiments [10], Screw Simulator experiments [46] and cone and plate viscometer experiments. Maddock screw-freezing experiments were performed to visualize the melting profile along the single-screw extruder channel. The melting profiles obtained for different materials and different screw geometry configurations were then compared with the corresponding simulation results on melting and flow in single-screw extruders. Screw Simulator experiments were performed to collect the shear stress and melting flux data which helped understanding the behavior of a polymer during its flow in single-screw extruders. Cone and plate viscometer experiments were performed to obtain the shear viscosity data which is needed in the simulations. The details of these three experiments, namely, Maddock screw-freezing experiments, Screw 
simulator experiments and cone and plate viscometer experiments, are given in the next three sections along with the experimental data.

\subsection{Maddock Screw-freezing Experiments}

In the present work, the Maddock screw-freezing experiments were performed for Acrylonitrile butadiene styrene (ABS) and Low-density polyethylene (LDPE) resins with different screw configurations in order to get further insight into the melting process in single-screw extruders. This technique, which was pioneered by Maddock [10] in the late 1950's, was performed by operating the extruder until a steady state was achieved. Screw rotation was then stopped and full air cooling to the barrel was applied to solidify the molten polymer in the screw channel. Next, the screw with the solidified polymer was pushed out of the barrel and examined by slicing the solidified polymer and polishing the cross-sectional surfaces. A small amount of black pigmented resin was added to the natural feedstock resin at a ratio of 1 to 100 to demarcate regions that were solid or molten prior to stopping the rotation of the screw. The regions in the screw that were molten were tinted with the pigment while the solid regions showed the natural color (white) of the main resin with small traces of pigmented particles. One sample cross-sectional view of the plastication process from a Maddock screw-freezing experiment is shown in Fig. 1.2 .

A highly instrumented $63.5 \mathrm{~mm}$ diameter single-screw extruder with a length-to-diameter (L/D) ratio of 21 was used to perform the Maddock screw-freezing experiments. Five conventional, single-flighted, square-pitch screws were used in the experiments. Two main screw geometry parameters which affect the melting process are the compression ratio and compression rate, which are defined by the following equations for screws with a constant lead length: 


$$
\begin{aligned}
\text { Compression Ratio }=\frac{H}{h} \\
\text { Compression Rate }=\frac{(H-h) \sin \theta_{b}}{M} \\
\tan \theta_{b}=\frac{L}{\pi D}
\end{aligned}
$$

where $h$ and $H$ are the screw channel depths in the metering and solids conveying sections, respectively, $M$ is the axial length of the compression section, $L$ is the lead length, $D$ is the inside barrel diameter, and $\theta_{b}$ is the helix angle at the barrel wall. Hereafter, the screws used in the experiments are named such that the first three numbers indicate the lengths of the solids conveying, compression and metering sections in terms of screw diameters and the last number shows the compression ratio of the screw. For example, a screw named $6-8-7 C R 2.0$ had six diameters of a solids conveying section, eight diameter long compression section, and a seven diameter long metering section with a 2.0 compression ratio. Various dimensions of the screws used in the experiments are given in Table 2.1. All the screws used had a constant metering channel depth of $3.18 \mathrm{~mm}$. For these screws, the specific rotational rate, which is defined as the flow rate per screw rotation based upon the drag flow of the polymer melt in the metering section, was identical and calculated at $0.86 \mathrm{~kg} /(\mathrm{h} \mathrm{rpm})$. Four of the screws used had the same length for the solids conveying, compression and metering sections with different compression ratios. In order to investigate the effect of the compression rate, the fifth screw had a shorter compression section and a longer metering section with a compression ratio of 2.8 .

For all screw-freezing experiments reported in this dissertation, resin entered the hopper at room temperature, the region of the barrel near the hopper was water cooled and the screw speed was set to $60 \mathrm{rpm}$. Discharge temperature was measured by a hand-held thermocouple. The axial pressure profiles data along the screw channel were 
Table 2.1: Various dimensions of the screws used in the screw-freezing experiments.

\begin{tabular}{|c|c|c|c|c|c|}
\hline Screw: & $6-8-7 \mathrm{CR} 2.0$ & 6-8-7CR2.4 & 6-8-7CR2.8 & 6-4-11CR2.8 & 6-8-7CR3.2 \\
\hline Compression ratio & 2.0 & 2.4 & 2.8 & 2.8 & 3.2 \\
\hline Compression rate & 0.00190 & 0.00266 & 0.00342 & 0.00684 & 0.00418 \\
\hline \multicolumn{6}{|l|}{ Length (screw diameters) } \\
\hline solids conveying section & 6 & 6 & 6 & 6 & 6 \\
\hline compression section & 8 & 8 & 8 & 4 & 8 \\
\hline metering section & 7 & 7 & 7 & 11 & 7 \\
\hline \multicolumn{6}{|l|}{ Channel depth (mm) } \\
\hline solids conveying section & 6.35 & 7.62 & 8.89 & 8.89 & 10.16 \\
\hline metering section & 3.18 & 3.18 & 3.18 & 3.18 & 3.18 \\
\hline Flight width perpendicular to the flight (mm) & 7.94 & 7.94 & 7.94 & 6.60 & 7.94 \\
\hline Flight clearance $(\mathrm{mm})$ & 0.07 & 0.07 & 0.07 & 0.07 & 0.07 \\
\hline \multicolumn{6}{|l|}{ Flight radii (mm) } \\
\hline pushing flight (solids conveying-metering) & $6.35-6.35$ & $7.62-7.62$ & $6.35-6.35$ & $7.87-3.05$ & $9.14-9.14$ \\
\hline trailing flight (solids conveying-metering) & $6.35-6.35$ & $7.62-7.62$ & $9.53-9.53$ & 7.87-3.05 & $9.14-9.14$ \\
\hline
\end{tabular}

obtained from the pressure transducers located at various axial positions along the axis of the barrel. The ABS resin used for the experiments was dried prior to the operation. For all screw-freezing experiments in which the ABS resin were used, the barrel temperatures in the solids conveying, compression, and metering zone were set at 200, 230, and 250 ${ }^{\circ} \mathrm{C}$, respectively, For the experiment with a screw configuration of 6-8-7CR2.8 in which the LDPE resin was used, the extruder was operated at barrel temperatures of $150{ }^{\circ} \mathrm{C}$ for the solids conveying zone, $175^{\circ} \mathrm{C}$ for the compression zone, and $200{ }^{\circ} \mathrm{C}$ for the metering zone.

\subsubsection{Experimental Results and Discussion}

With the experimental conditions discussed in Section 2.1, extrusion rate, specific rate, discharge pressure and discharge temperature for the screw-freezing experiments of $A B S$ and LDPE resins are summarized in Table 2.2. The axial pressure profile data obtained from the pressure transducers positioned at various locations along the barrel are shown in Fig. 2.1 for ABS and LDPE resins. The cross-sectional views of the plastication process from the screw-freezing experiments are shown in Figs. $2.2-2.7$. The numbers along 
Table 2.2: Experimental conditions obtained from the screw-freezing experiments.

\begin{tabular}{ccccccc}
\hline Screw & Resin & $\begin{array}{c}\text { Extrusion rate } \\
(\mathrm{kg} / \mathrm{h})\end{array}$ & $\begin{array}{c}\text { Specific rate } \\
(\mathrm{kg} /[\mathrm{h} \mathrm{rpm}])\end{array}$ & $\begin{array}{c}\text { Discharge } \\
\text { pressure }(\mathrm{MPa})\end{array}$ & $\begin{array}{c}\text { Discharge } \\
\text { temperature }\left({ }^{\circ} \mathrm{C}\right)\end{array}$ & $\begin{array}{c}\text { Screw speed } \\
(\mathrm{rpm})\end{array}$ \\
\hline 6-8-7CR2.0 & ABS & 61.1 & 1.02 & 16.3 & 261.9 & 60 \\
$6-8-7 \mathrm{CR} 2.4$ & ABS & 60.3 & 1.01 & 15.9 & 261.5 & 60 \\
6-8-7CR2.8 & ABS & 59.7 & 1.00 & 15.4 & 263.6 & 60 \\
6-8-7CR2.8 & LDPE & 45.6 & 0.76 & 10.5 & 203.7 & 60 \\
6-4-11CR2.8 & ABS & 56.2 & 0.94 & 14.1 & 261.9 & 60 \\
6-8-7CR3.2 & ABS & 56.7 & 0.95 & 14.9 & 261.0 & 60 \\
\hline
\end{tabular}

the cross-sections show the axial location of the cross-section as a multiple of the screw diameter. As shown in Figs. 2.2 - 2.7, Maddock's melting mechanism [10] was observed in all experiments. A thin layer of molten polymer between the hot barrel and compacted solid pellets was observed to accumulate to the active flight and the width of the melt pool gradually increased in the down-channel direction whereas that of the solid bed decreased. However, in all experiments, there was a sudden decrease in the width of the solid bed at axial distance of around 14 diameters. Beyond this point, the continuity of the solid bed was broken (solid bed break-up). It is also noted that the experimental results showed the tendency of the melt pool to penetrate under the solid bed on the screw surface.

For the experimental data shown in Figs. 2.2 - 2.6, Fig. 2.8 presents the variation of the solid fraction along the screw channel. The solid fraction for the ABS resin experiments presented in Fig. 2.8 was measured as a ratio of the solid bed area to the area of the screw channel at that cross-section. The solid fraction thus obtained was further normalized by multiplying it with the ratio of the current screw channel area to the screw channel area at the entrance of the compression section. Therefore, the values of the solid fraction in Fig. 2.8 show the percentage of the solid material remaining at a cross-section compared to the solid material at the entrance. The calculation of the solid fraction described above can be written as follows: 


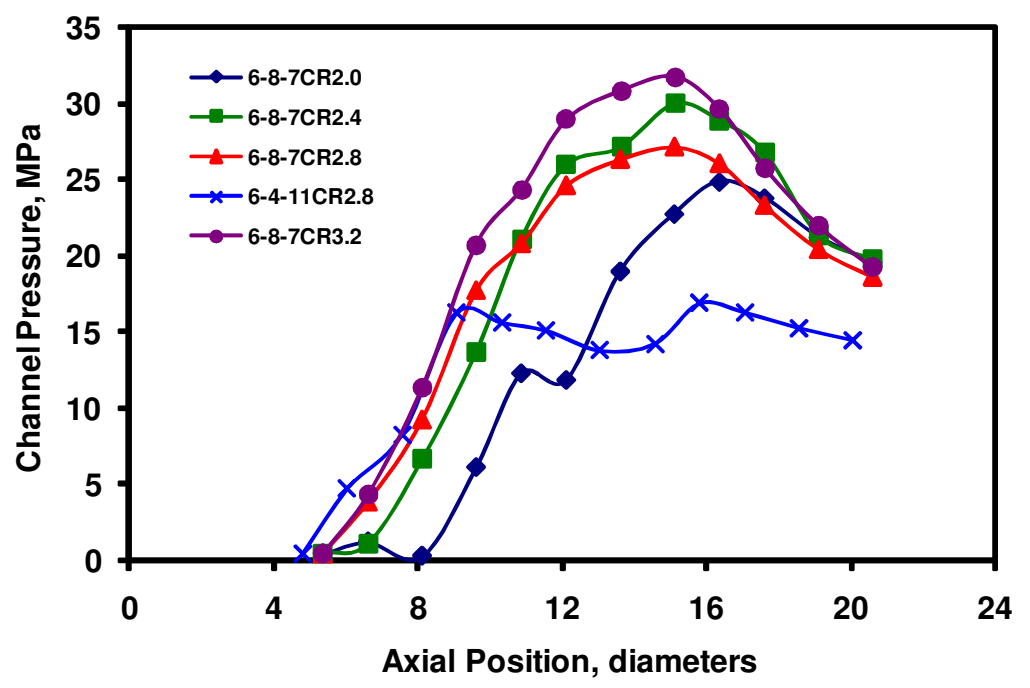

(a) ABS resin

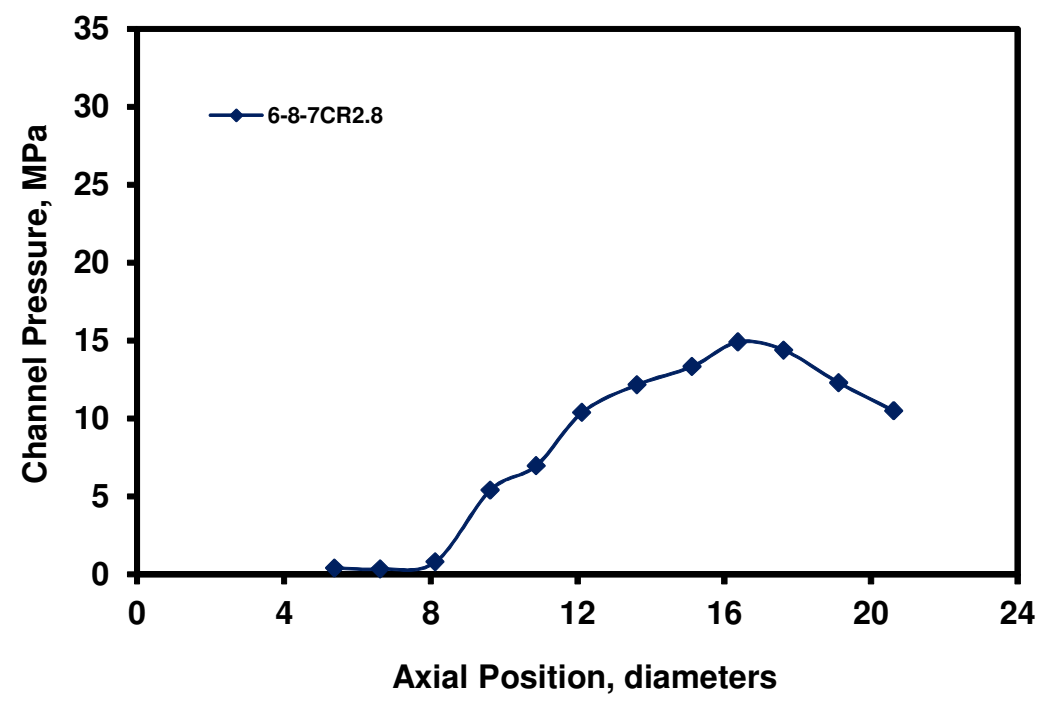

(b) LDPE resin

Figure 2.1: Axial pressure profile of the ABS and LDPE resins with different screw configurations running at a screw speed of $60 \mathrm{rpm}$. 


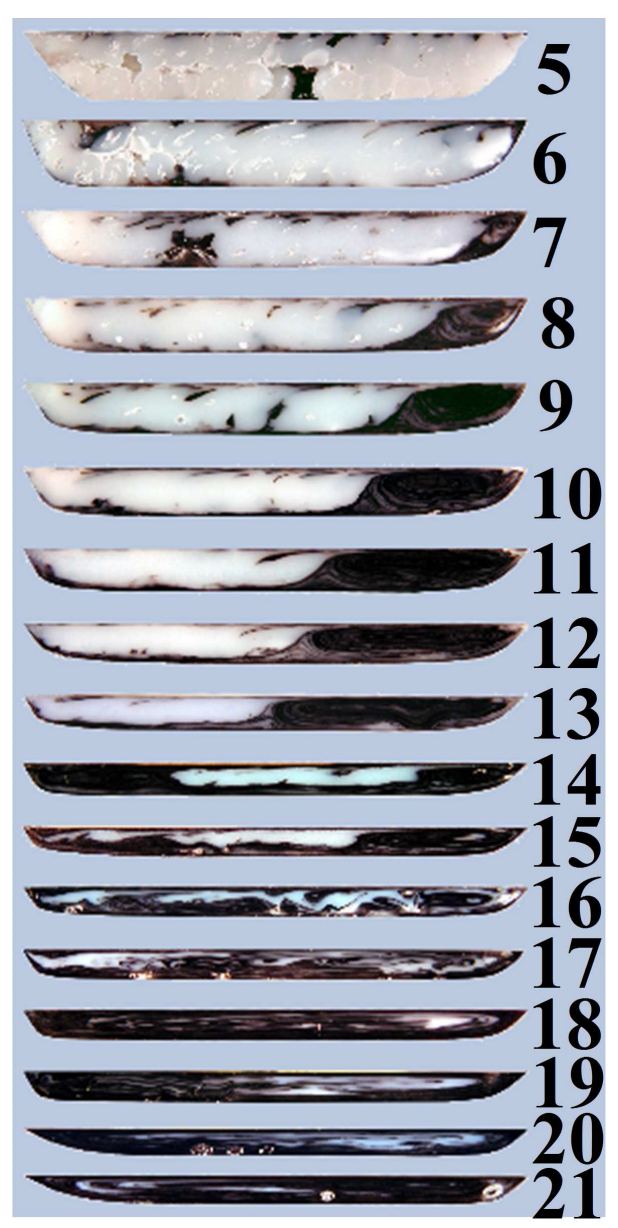

Figure 2.3: Cross-sections of the solidified polymer obtained from the solidification experiments of the ABS resin using the 6-8-7CR2.4 screw.
Figure 2.2: Cross-sections of the solidified polymer obtained from the solidification experiments of the $A B S$ resin using the 6-8-7CR2.0 screw.

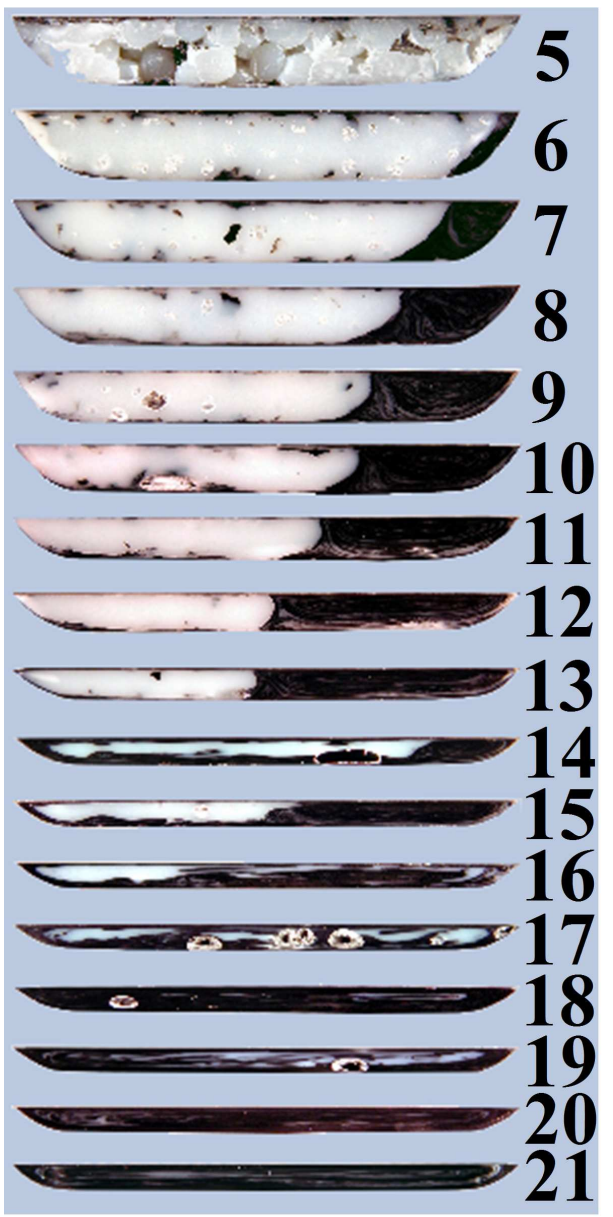




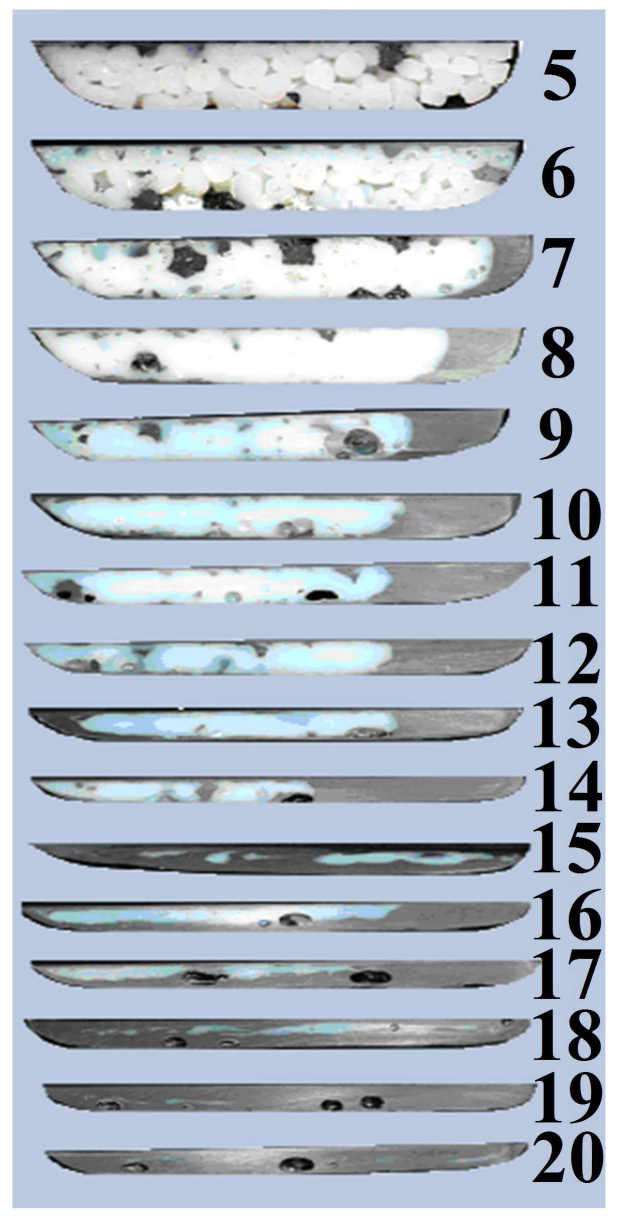

Figure 2.4: Cross-sections of the solidified polymer obtained from the solidification experiments of the $A B S$ resin using the 6-8-7CR2.8 screw.

Figure 2.5: Cross-sections of the solidified polymer obtained from the solidification experiments of the $A B S$ resin using the 6-4-11CR2.8 screw.

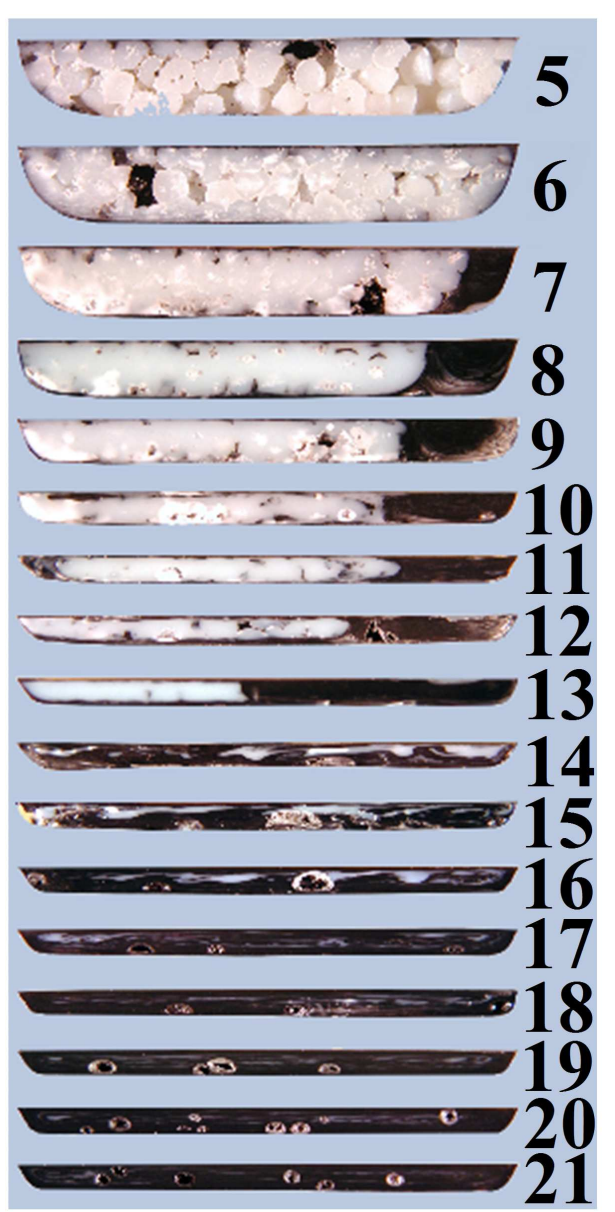




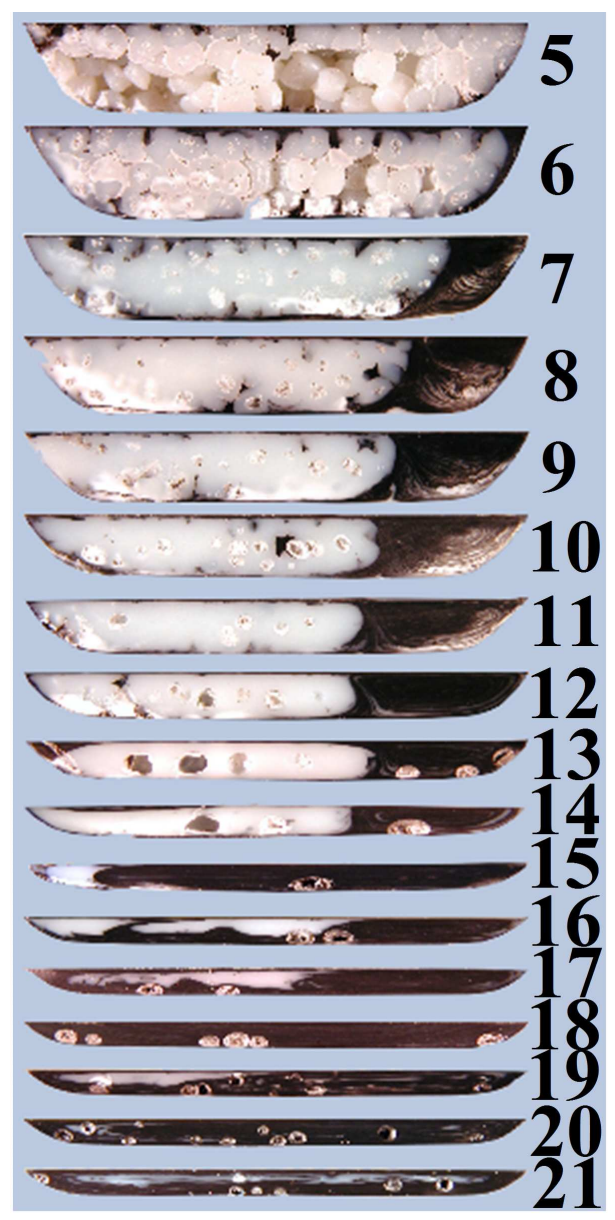

Figure 2.6: Cross-sections of the solidified polymer obtained from the solidification experiments of the $A B S$ resin using the 6-8-7CR3.2 screw.

Figure 2.7: Cross-sections of the solidified polymer obtained from the solidification experiments of the LDPE resin using the 6-8-7CR2.8 screw.

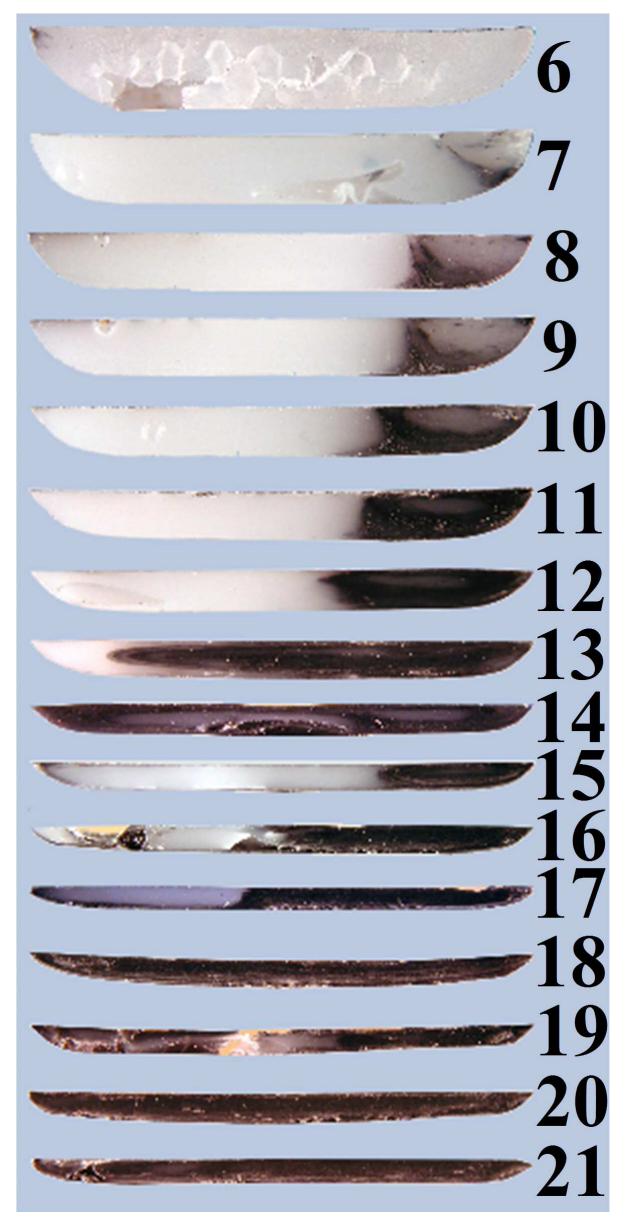




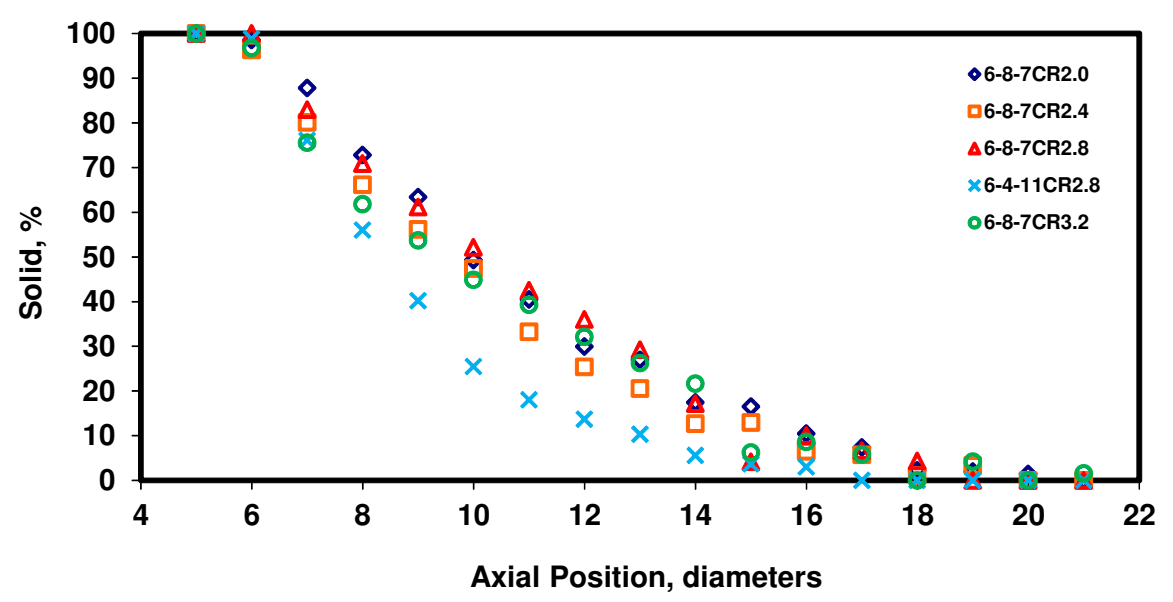

Figure 2.8: Experimental melting profiles of the $A B S$ resin obtained from the Maddock screw-freezing experiments for different screw configurations.

$$
\text { Solid fraction }=\frac{A_{\text {solid }}}{A_{\text {ent }}}
$$

where $A_{\text {solid }}$ is the solid bed area at that cross-section and $A_{\text {ent }}$ is the screw channel area at the entrance of the compression section.

The experimental data shown in Figs. $2.2-2.6$ and 2.8 indicate that if the axial length of the compression section is kept the same, a change in compression ratio has only a minor effect on the melting rate - the melting rate increased slightly with increasing compression ratio. However, for a fixed compression ratio, an increase in compression rate resulted in a faster melting rate.

\subsection{Screw Simulator Experiments}

Part of this section is from a preprint of an article published in [3] (See Appendix A for the copyright agreement). Frictional forces (for temperatures less than the melting or devitrification temperature) and viscous forces (for higher temperatures) have important roles on solids conveying and melting processes in plasticating single-screw 
extruders. These forces are related to the shear stresses at polymer-metal interfaces. For temperatures at which the frictional forces are the main factor for the shear stresses, it is experimentally difficult to obtain the shear stresses at the polymer-metal interface. The interpretation of the data has further complications due to the frictional energy dissipation at the polymer-metal interface. In Screw Simulator experiments, an instrument called the Screw Simulator $[46,66]$ was used to measure the forces resulting either from a frictional mechanism or viscous mechanism at a polymer-metal interface. These forces were then used to determine the shear stresses at a polymer-metal interface which allows the data to be presented continuously as a function of temperature. The first Screw Simulator was designed and built by Chung [46]. A modified version [66] of the Screw Simulator based upon Chung's work was used in this work to perform the experiments reported here. The schematic of the instrument is shown in Fig. 2.9. Resin pellets were placed in a $5.08 \times 2.54$ $\mathrm{cm}$ rectangular sample chamber $\left(12.9 \mathrm{~cm}^{2}\right.$ sample area). The sample was then forced onto a rotating roll with a fixed force, and the torque required to rotate the roll at a constant speed was measured to compute the parameters such as coefficient of dynamic friction and shear stress. The rotating roll had a radius and width of 15.24 and $10.16 \mathrm{~cm}$, respectively. It was fabricated from 4140 steel and the surface had a $1.27 \mathrm{~mm}$ hard-face coating of Colmonoy 56. Resin pellets were forced onto the rotating roll using a plunger and pneumatic system. More details about the equipment were given by Spalding et al. [66]. At temperatures less than melting temperature about $5 \mathrm{~g}$ of resin were poured into the sample chamber such as to provide a bed with a height of about $6 \mathrm{~mm}$. This low height minimizes the frictional losses on the side walls of the sample chamber and thus allows the full transfer of the force from the plunger to the pellets at the roll surface. Slightly more resin was used at higher temperatures.

A linear variable-differential transformer (LVDT) and a high-speed data acquisition system were used to measure the thickness of the sample in the chamber. The rate 


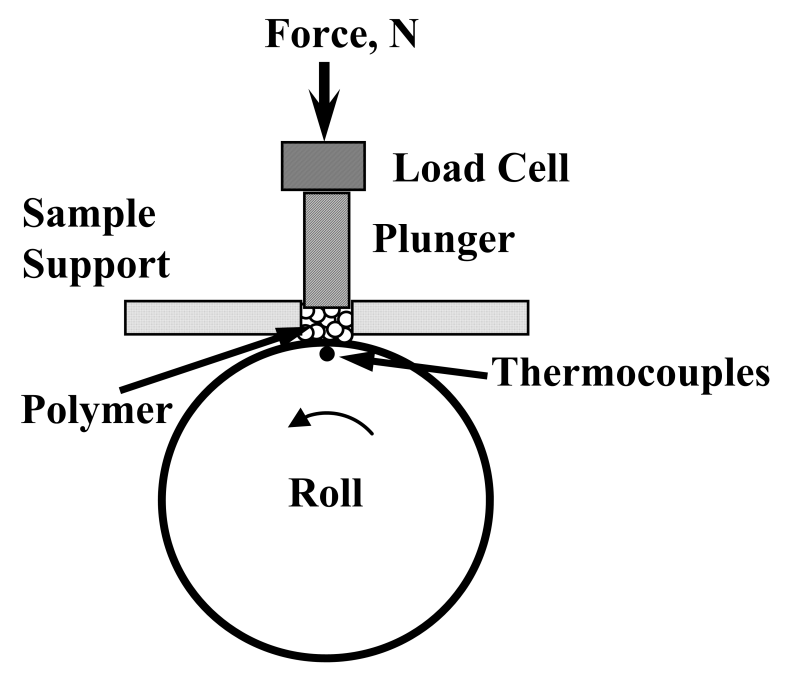

Figure 2.9: Schematic of the Screw Simulator.

of change of this thickness was used to compute the melting flux of the polymer. The computation schemes for calculating the coefficient of dynamic friction, shear stress, and melting flux were given by Spalding et al. [8] and the equations for these calculations can be found in Section 2.2.2. The roll temperature can be adjusted in the range of ambient temperature to $300{ }^{\circ} \mathrm{C}$, and it was measured using three thermocouples located along the width of the roll and $3 \mathrm{~mm}$ away from the sliding interface. For temperatures less than the melting or devitrification temperature an implicit finite difference technique was used to predict the polymer-metal interface temperature based on the measured metal temperature and the amount of heat generated by friction at the interface [66]. This computed polymer-metal interface temperatures were reported in Section 2.2.3 for temperatures less than the melting temperature. Thermocouple measurements were reported in Section 2.2.3 for those experiments involving melting.

\subsubsection{Resins}

Low density polyethylene (LDPE), linear low density polyethylene (LLDPE), acrylonitrile butadiene styrene (ABS), high-impact polystyrene (HIPS), and three different 
Table 2.3: Flow properties and test conditions for the resins used in the Screw Simulator experiments.

\begin{tabular}{|l|c|c|}
\hline Resin & Flow, dg/min & Conditions \\
\hline \hline LDPE & 2.0 & $190^{\circ} \mathrm{C}, 2.16 \mathrm{~kg}$ \\
\hline LLDPE & 1.0 & $190^{\circ} \mathrm{C}, 2.16 \mathrm{~kg}$ \\
\hline ABS & 2.5 & $230^{\circ} \mathrm{C}, 3.8 \mathrm{~kg}$ \\
\hline HIPS & 2.8 & $200^{\circ} \mathrm{C}, 5 \mathrm{~kg}$ \\
\hline PC-3 & 3.0 & $300^{\circ} \mathrm{C}, 1.2 \mathrm{~kg}$ \\
\hline PC-6 & 6.0 & $300^{\circ} \mathrm{C}, 1.2 \mathrm{~kg}$ \\
\hline PC-22 & 22.0 & $300^{\circ} \mathrm{C}, 1.2 \mathrm{~kg}$ \\
\hline
\end{tabular}

polycarbonate $(P C)$ resins were used for the Screw Simulator experiments. The three polycarbonate resins were labeled as PC-3, PC-6, and PC-22 with the melt flow rates (MFRs) of 3, 6, and $22 \mathrm{dg} / \mathrm{min}$, respectively. All resins were manufactured by The Dow Chemical Company. The melt flow indices for all resins are shown in Table 2.3. Coefficients of dynamic friction, shear stresses at the interface, and melting fluxes for similar resins have been reported previously in the literature $[8,39,46,53,66-70]$, but the earlier data were not as comprehensive as those reported here. In particular, most of the data available in the literature did not extend to high temperatures. The Screw Simulator experiments with LDPE, LLDPE, ABS and HIPS resins were performed to investigate the shear stress and melting flux characteristics of semi-crystalline (LDPE, LLDPE) and amorphous (ABS, HIPS) polymers whereas three PC resins with different MFRs were used for the Screw Simulator experiments to investigate how MFR affects the shear stress and melting flux.

\subsubsection{Calculation of Shear Stresses and Melting Fluxes}

The shear stresses at the polymer-metal interface and melting fluxes were measured for all resins (LDPE, LLDPE, ABS, HIPS, and PC) at a fixed pressure of $0.7 \mathrm{MPa}$ and at roll velocities of $7.6,15.2,30.5$, and $61 \mathrm{~cm} / \mathrm{s}$. These data are important for better understanding of the solids conveying and melting processes in plasticating single-screw 
extruders. Equations 2.5 through 2.7 were used for computing the shear stress $(\tau)$ due to solid-state friction, shear stress due to a viscous mechanism, and melting flux $\left(F_{m}\right)$ of the resins, respectively. Shear stress due to solid-state friction is given by the equation:

$$
\tau=\frac{f N}{A}
$$

where $N$ is the force applied to the sample as shown in Fig. 2.9, $A$ is the cross-sectional area of the sample chamber $\left(12.9 \mathrm{~cm}^{2}\right)$, and $f$ is the coefficient of dynamic friction. In this equation, it was assumed that the pellets completely fill the area of sample chamber. The real contact area between the resin pellets and the rotating roll is not known. This assumption is likely acceptable since the contact area in an actual extrusion process will be similar to that occurring in the sample chamber of the Screw Simulator. Shear stress due to a viscous mechanism is written as:

$$
\tau=\frac{T R}{A}
$$

where $T$ is the measured torque, $R$ is the radius of the roll $(15.24 \mathrm{~cm})$, and $A$ is the cross-sectional area of the sample chamber. Melting flux of the resin can be computed with the equation:

$$
F_{m}=\frac{(d h / d t) m}{A h_{0}}
$$

where $d h / d t$ is the rate of change of the sample height, $m$ is the initial sample mass, and $h_{0}$ is the initial sample height. The flux values reported in this study were collected after the process achieved a steady state. At the start of the experiments, some of the molten resin may have flowed between the voids in the pellets. After steady state was achieved, however, the temperature of the pellets near the interface quickly increased and the pellets 
deformed, eliminating the voids. This phenomenon of void elimination was apparent by stopping the experiment mid way through the data collection process and observing the sample chamber.

\subsubsection{Experimental Results and Discussion}

\section{LDPE, LLDPE, ABS, and HIPS resins ${ }^{1}$}

The shear stresses for LDPE, LLDPE, ABS, and HIPS resins as a function of temperature and velocity are shown in Figs. 2.10 through 2.13, respectively. The data for these resins were consistent with those presented previously $[8,39,46,53,66-70]$. For the LDPE resin, as indicated in Fig. 2.10, the shear stress was nearly constant up to a temperature of about $70{ }^{\circ} \mathrm{C}$ depending on the velocity and then decreased as the temperature was increased further. This decrease in shear stress was rapid up to $110{ }^{\circ} \mathrm{C}$ compared to the decrease at higher temperatures. For instance at a roll surface velocity of $15.2 \mathrm{~cm} / \mathrm{s}$, the shear stress between the rubbing polymer and the metal surface was nearly constant at $0.44 \mathrm{MPa}$ at temperatures up to $70{ }^{\circ} \mathrm{C}$. As the temperature increased to $110{ }^{\circ} \mathrm{C}$, the shear stress decreased rapidly to $0.22 \mathrm{MPa}$, and a further increase in temperature to $230{ }^{\circ} \mathrm{C}$ resulted in a shear stress of $0.1 \mathrm{MPa}$. It is also interesting to note that at a temperature of $110{ }^{\circ} \mathrm{C}$ the shear stresses were essentially the same for all velocities. This temperature is close to the melting temperature of LDPE resin, which is $111^{\circ} \mathrm{C}$, and below this temperature the shear stress is controlled by solid-state (frictional) forces whereas viscous forces control the shear stress at temperatures higher than the melting temperature. Thus, as the temperature is increased above the melting temperature, the shear stress is influenced by the non-Newtonian behavior of the shear viscosity of the resin and depends directly on velocity gradients. Therefore, in the region above the melting temperature, the shear

\footnotetext{
${ }^{1}$ This section is from a preprint of an article published in [3]. See Appendix A for the copyright agreement.
} 


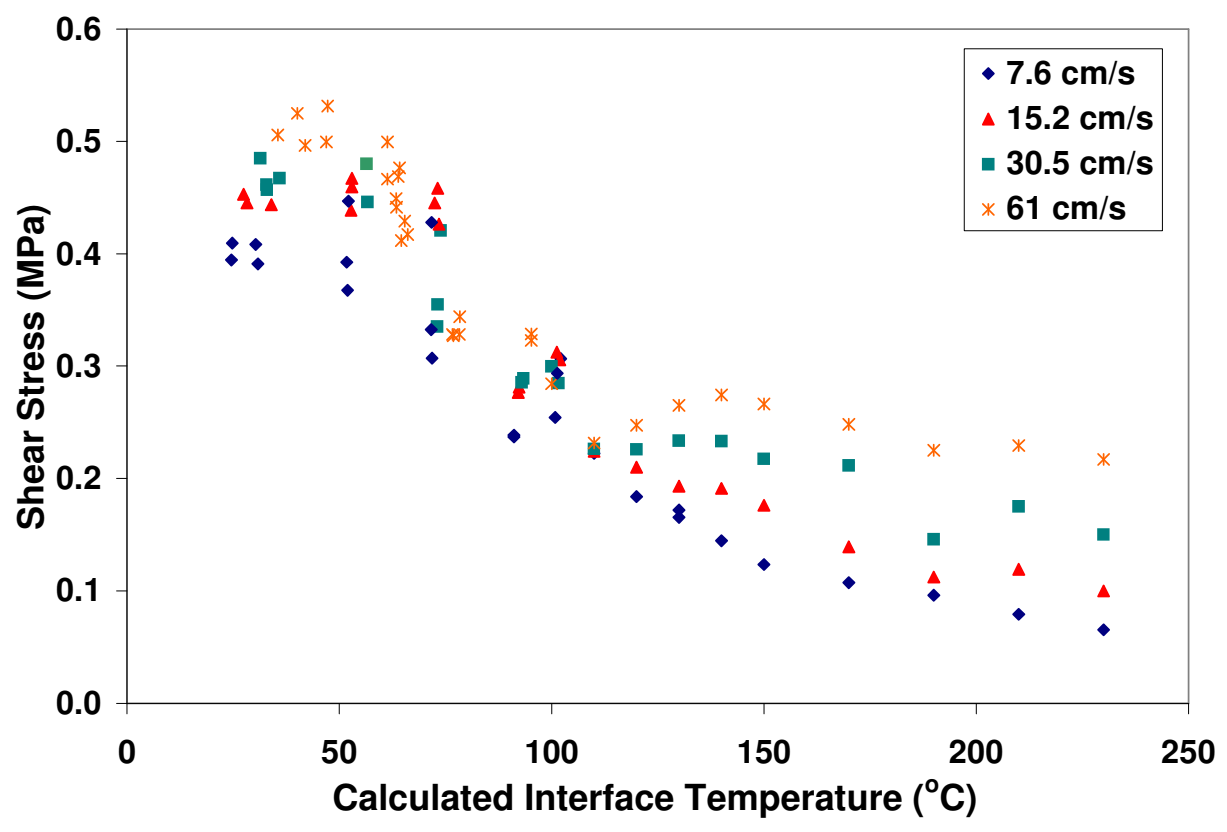

Figure 2.10: Shear stress at the interface for the LDPE resin at $0.7 \mathrm{MPa}$ as a function of temperature and velocity.

stress is expected to increase with increasing velocity. This expected increase in shear stress was observed in Fig. 2.10 for the temperatures higher than $110{ }^{\circ} \mathrm{C}$. For the LDPE resin, a thin melt film was observed on the roll surface for roll temperature of $150{ }^{\circ} \mathrm{C}$ and above.

For the LLDPE resin, as shown in Fig. 2.11, the shear stress at the interface exhibited similar trends as those for the LDPE resin with some subtle differences. In Fig. 2.11, for the LLDPE resin, the shear stress decreased with increasing temperature at a smaller rate than that for the LDPE resin in Fig. 2.10. The difference in stress behavior between the LDPE and LLDPE resin at very low temperatures may be due to low levels of additives in the LLDPE resin. Moreover, the shear stresses for the LLDPE resin at all velocities remained nearly constant as the temperature was increased above $150{ }^{\circ} \mathrm{C}$. The interaction between the shear viscosity, shear rate, and the thickness of the melt film between the solids and the roll surface is complex, and these interactions were likely contributing to this 


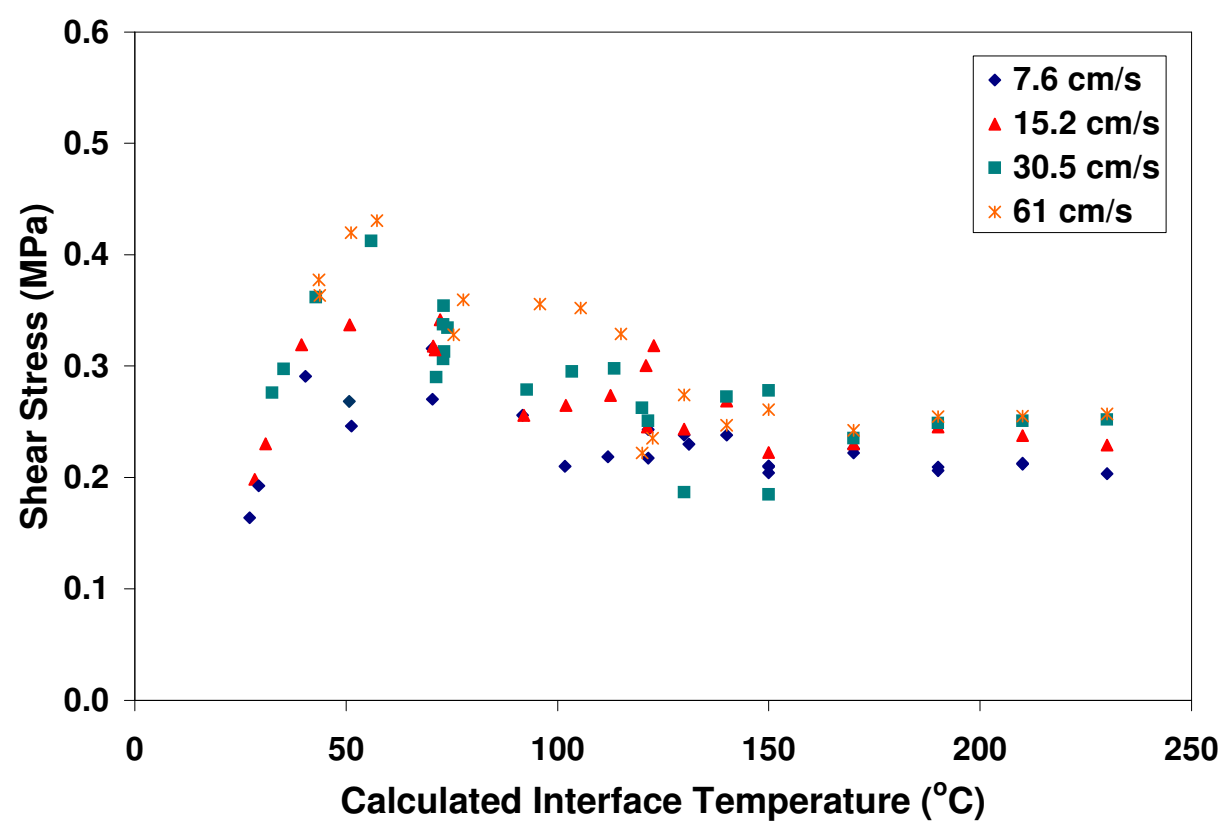

Figure 2.11: Shear stress at the interface for the LLDPE resin at $0.7 \mathrm{MPa}$ as a function of temperature and velocity.

difference in the behavior between the LDPE and LLDPE resins. At the same temperature, the shear stresses were still increasing with increasing velocity for temperatures where a viscous mechanism controls the shear stress.

The shear stress for the ABS resin at a pressure of $0.7 \mathrm{MPa}$ had a different behavior than that for the LDPE and LLDPE resins. As shown in Fig. 2.12, for the ABS resin, the shear stress values had a minimum around $90{ }^{\circ} \mathrm{C}$ and then reached a maximum around $160{ }^{\circ} \mathrm{C}$ for all velocities. This behavior is important to the extrusion process which will be discussed later. As indicated in Fig. 2.12, the shear stress values decreased as the temperature increased to $90{ }^{\circ} \mathrm{C}$. In the range of 90 to $160{ }^{\circ} \mathrm{C}$, the shear stress increased approximately three times with increasing temperature. This increase in shear stress was higher for lower roll velocities. For instance, at the roll surface velocity of $7.6 \mathrm{~cm} / \mathrm{s}$, the shear stress at the interface was around $0.11 \mathrm{MPa}$ at $90{ }^{\circ} \mathrm{C}$, while at $160{ }^{\circ} \mathrm{C}$ the shear stress was $0.44 \mathrm{MPa}$, an increase of four times in shear stress. At temperatures higher 


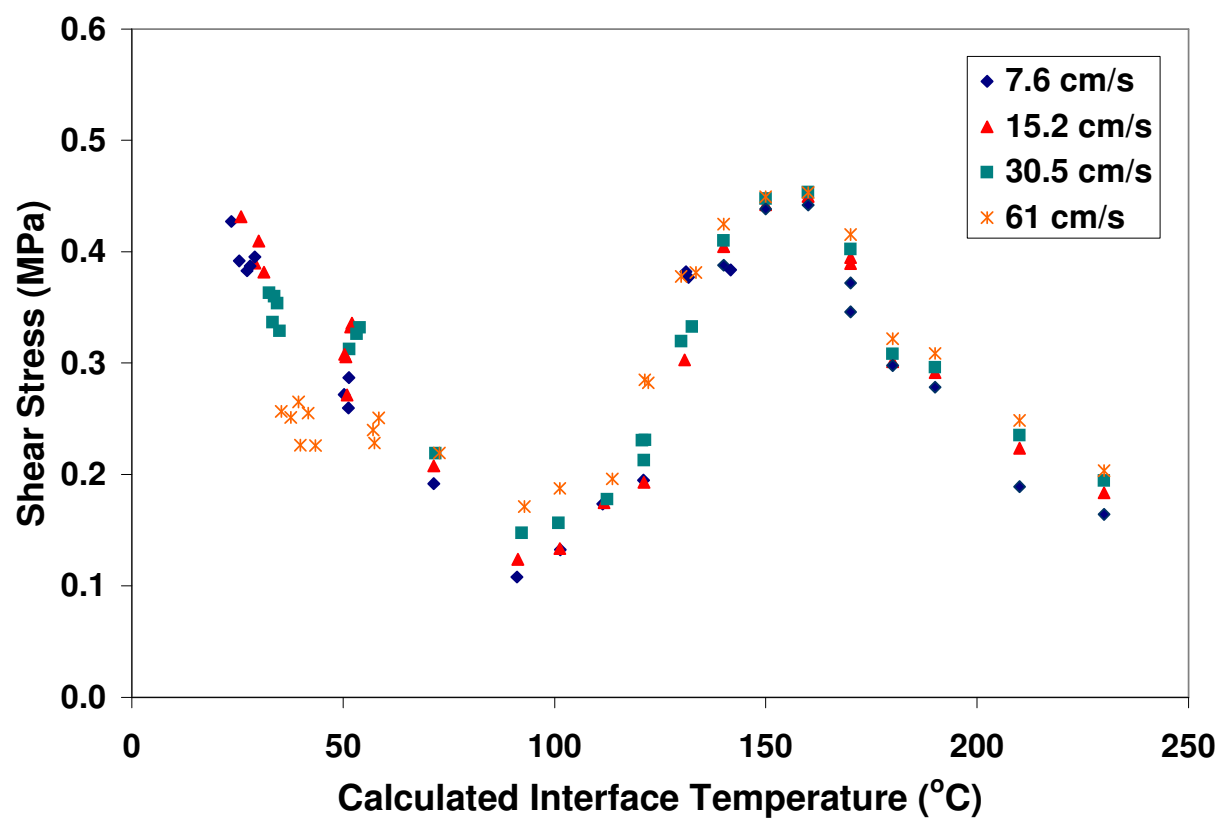

Figure 2.12: Shear stress at the interface for the ABS resin at $0.7 \mathrm{MPa}$ as a function of temperature and velocity.

than $160{ }^{\circ} \mathrm{C}$, the shear stress decreased as the temperature was increased. This decrease in shear stress for $\mathrm{ABS}$ resin is similar to that for LDPE resin above $110^{\circ} \mathrm{C}$, but the rate of reduction for $A B S$ resin is much higher. The shear stress for the $A B S$ resin also increased with increasing velocity but at a lower rate than that for the LDPE resin.

The shear stress for the HIPS resin showed trends that were similar to those for the ABS resin. As shown in Fig. 2.13, the shear stress had a maximum at about $130{ }^{\circ} \mathrm{C}$ and decreased with a further increase in temperature. For temperatures higher than 130 ${ }^{\circ} \mathrm{C}$, the dependency of the shear stress on velocity was similar to that for the ABS resin. At temperatures higher than $170{ }^{\circ} \mathrm{C}$, the shear stress decreased at a similar rate for all velocities. For temperatures below $110{ }^{\circ} \mathrm{C}$, however, the shear stress for HIPS resin was relatively constant and did not have minimum values like those for the ABS resin.

At temperatures higher than the melting (or devitrification) temperature, it is clear from Figs. 2.10 through 2.13 that the shear stress depends on the shear rate (or velocity 


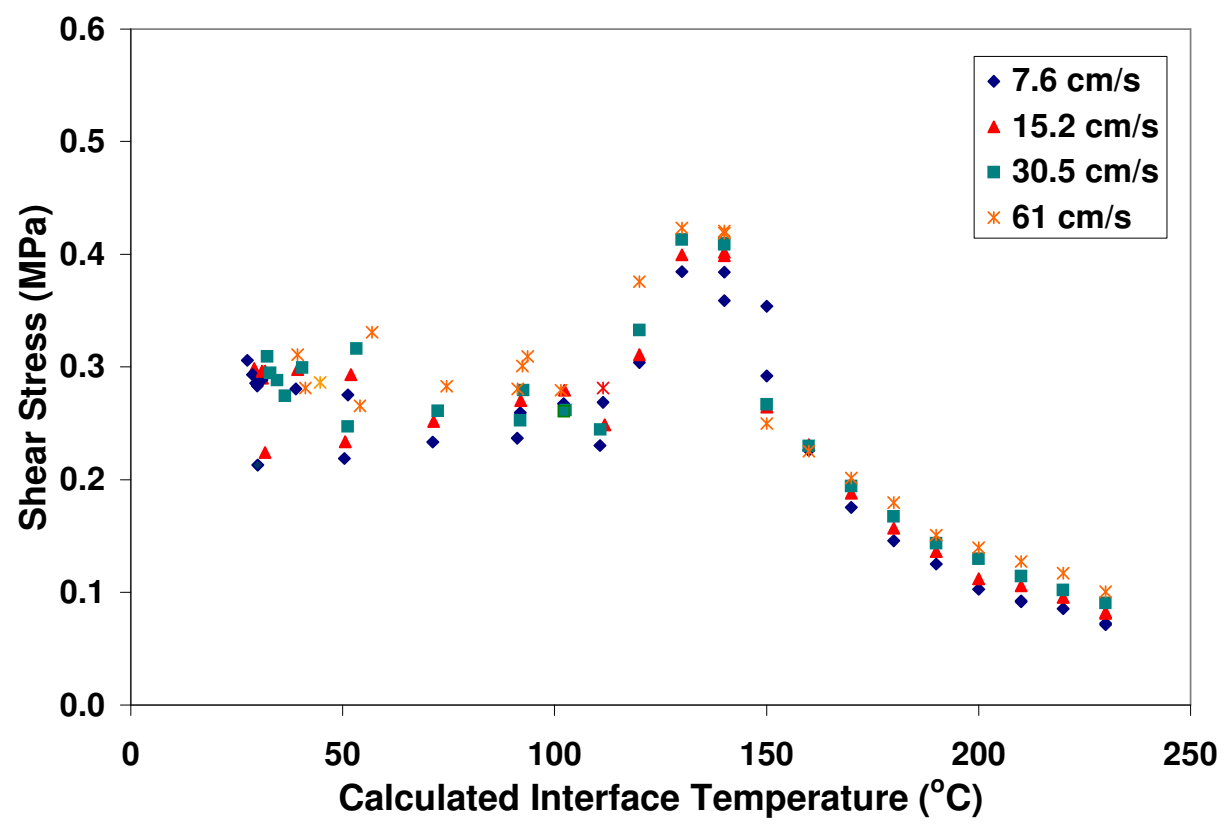

Figure 2.13: Shear stress at the interface for the HIPS resin at $0.7 \mathrm{MPa}$ as a function of temperature and velocity.

gradient). As shown by these figures, the shear stresses increase with increasing velocity or increasing velocity gradient along the melt film. Thus, it may be stated that above these temperatures, shear stresses depend mainly on viscous forces. As previously mentioned, the interaction of the shear rate, viscosity, temperature, and melt film thickness is complex and beyond the scope of this research. Below the melting or devitrification temperature the shear stresses are determined by a frictional mechanism. For amorphous resins, in the intermediate regions, where the resin is considered as neither solid nor liquid, both mechanisms influence the shear stress. Crystalline polymers do not have this intermediate region since the crystalline polymers have a distinct melting point. For temperatures in the range of 100 to $170{ }^{\circ} \mathrm{C}$ for $\mathrm{ABS}$ and HIPS resins, which are amorphous resins in nature, both mechanisms likely affect the shear stress. For these resins, the peak in the shear stress values is likely due to the bonding of the rubbery state polymer resin to the metal surface. 
The melting flux data for the four resins as a function of temperature and velocity are given in Figs. 2.14 through 2.17. The minimum temperature in each of the Figs. 2.14 through 2.17 is around the mechanical melting temperature of the resins where a significant portion of the thermal energy for melting originates from a mechanical energy dissipative process $[8,42]$. However, it was observed in the Screw Simulator experiments that the temperatures at which a thin melt film formed over the roll surface was much higher than the mechanical melting temperature. For instance, for ABS and HIPS resins melt films developed on the roll surface at temperatures of 170 and $150{ }^{\circ} \mathrm{C}$, respectively. The mechanical melting temperatures for the ABS and HIPS resins were 130 and $120^{\circ} \mathrm{C}$, respectively.

It is evident from the Fig. 2.14 that the melting flux for the LDPE resin depends on the velocity and roll temperature. For roll surface velocities of 7.6 and $15 \mathrm{~cm} / \mathrm{s}$ and temperatures higher than $150^{\circ} \mathrm{C}$, the melting flux was almost independent of temperature. For higher velocities the melting flux increased as the temperature was increased. For the LLDPE resin in Fig. 2.15, an increase in melting flux was observed with increasing temperature for all velocities. For the ABS (Fig. 2.16) and HIPS (Fig. 2.17) resins, the melting fluxes were nearly the same and were essentially constant with increasing temperature for temperatures higher than about $160{ }^{\circ} \mathrm{C}$. At the same temperature the melting flux for the four resins increased with increasing velocity.

A comparison of the melting fluxes for these four resins provides an interesting insight into the melting process in plasticating single-screw extruders. Such a comparison of melting fluxes at the roll surface velocity of $61 \mathrm{~cm} / \mathrm{s}$ is shown in Fig. 2.18. In Fig. 2.18, at temperature of $210{ }^{\circ} \mathrm{C}$, the melting fluxes for LDPE, LLDPE, ABS, and HIPS resins were at $0.624,0.359,0.206$, and $0.184 \mathrm{~kg} /\left(\mathrm{m}^{2} \mathrm{~s}\right)$, respectively. Thus, the melting fluxes were similar for the ABS and HIPS resins. The LLDPE resin, however, had a higher melting flux than the ABS and HIPS resins, and the LDPE resin had a melting flux that 


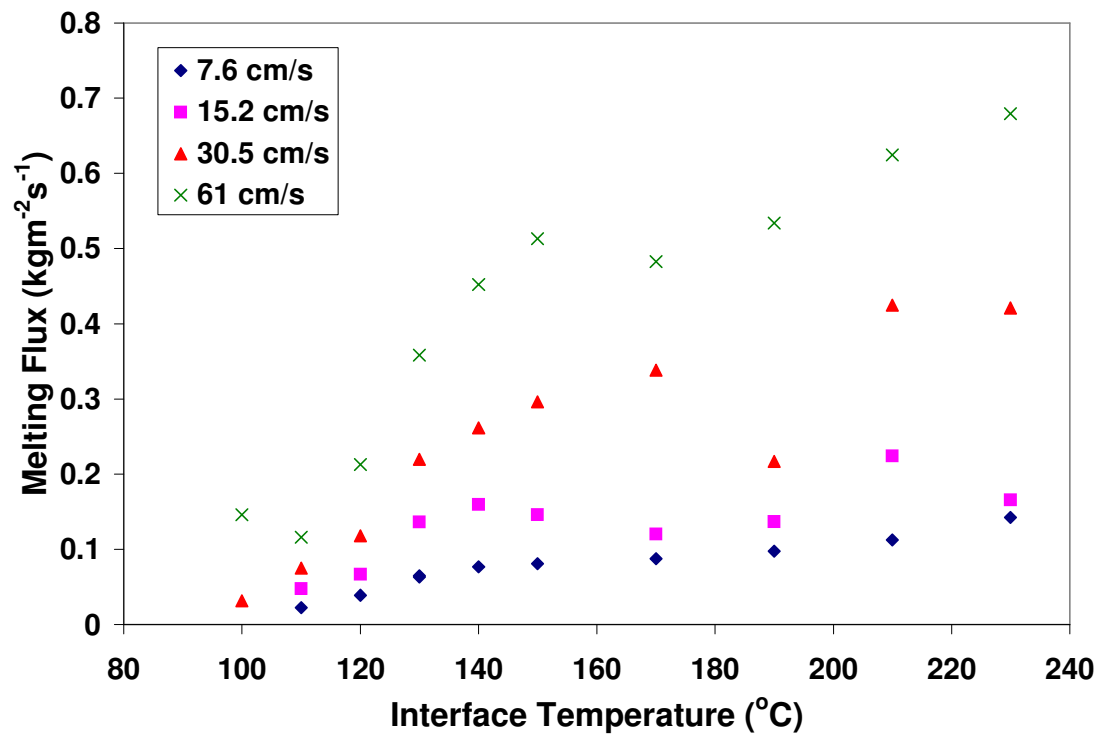

Figure 2.14: Melting flux for the LDPE resin at $0.7 \mathrm{MPa}$ as a function of temperature and velocity.

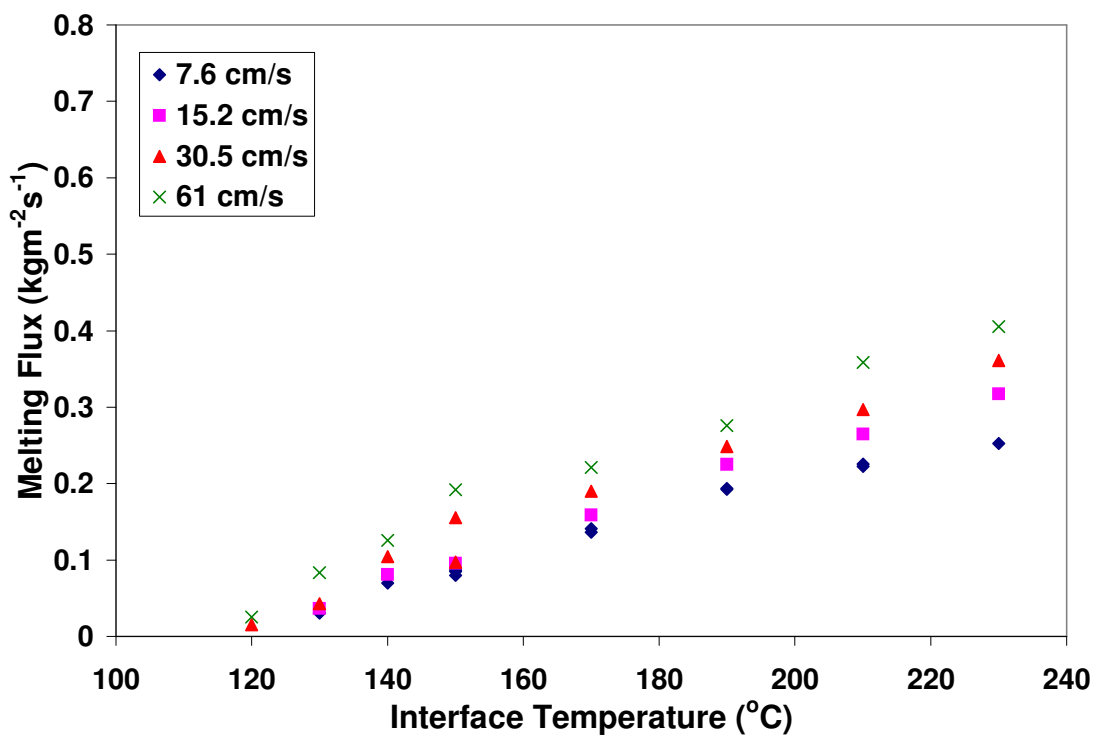

Figure 2.15: Melting flux for the LLDPE resin at $0.7 \mathrm{MPa}$ as a function of temperature and velocity. 


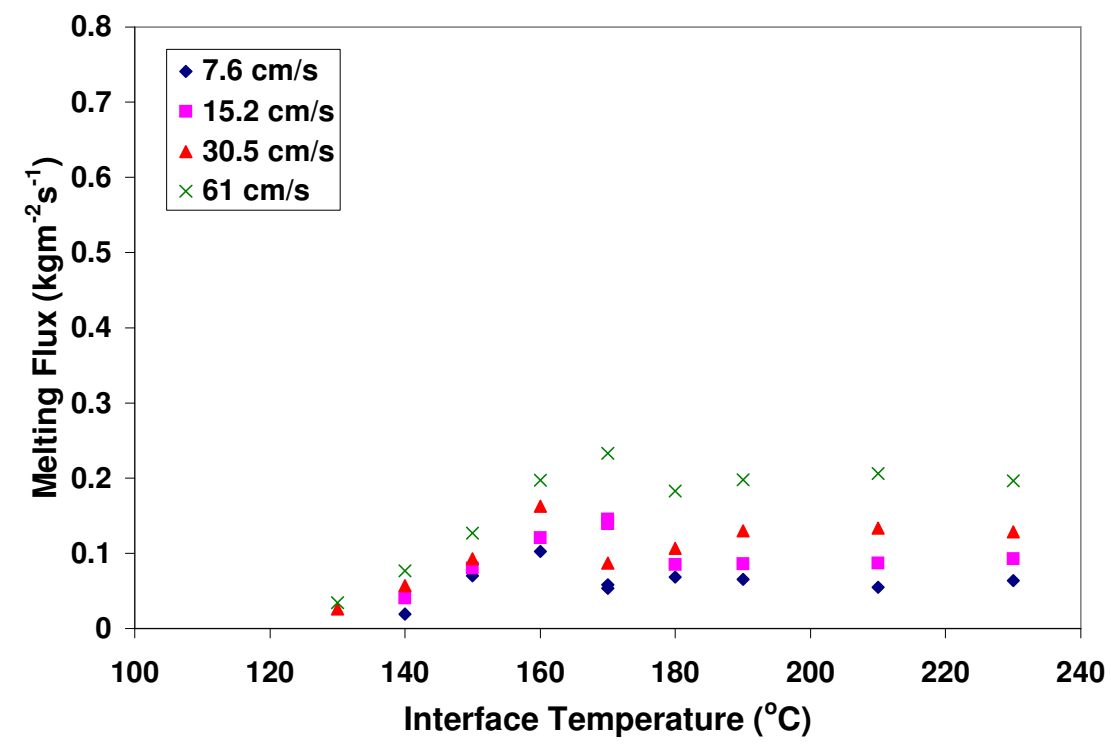

Figure 2.16: Melting flux for the $\mathrm{ABS}$ resin at $0.7 \mathrm{MPa}$ as a function of temperature and velocity.

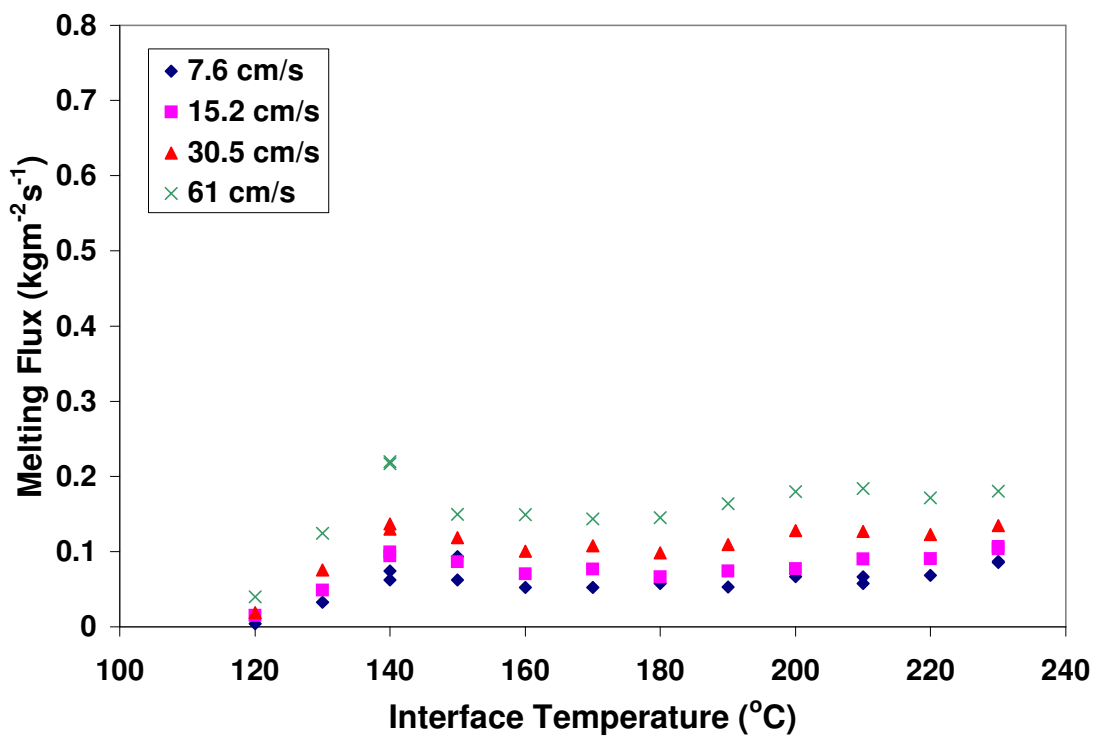

Figure 2.17: Melting flux for the HIPS resin at $0.7 \mathrm{MPa}$ as a function of temperature and velocity. 


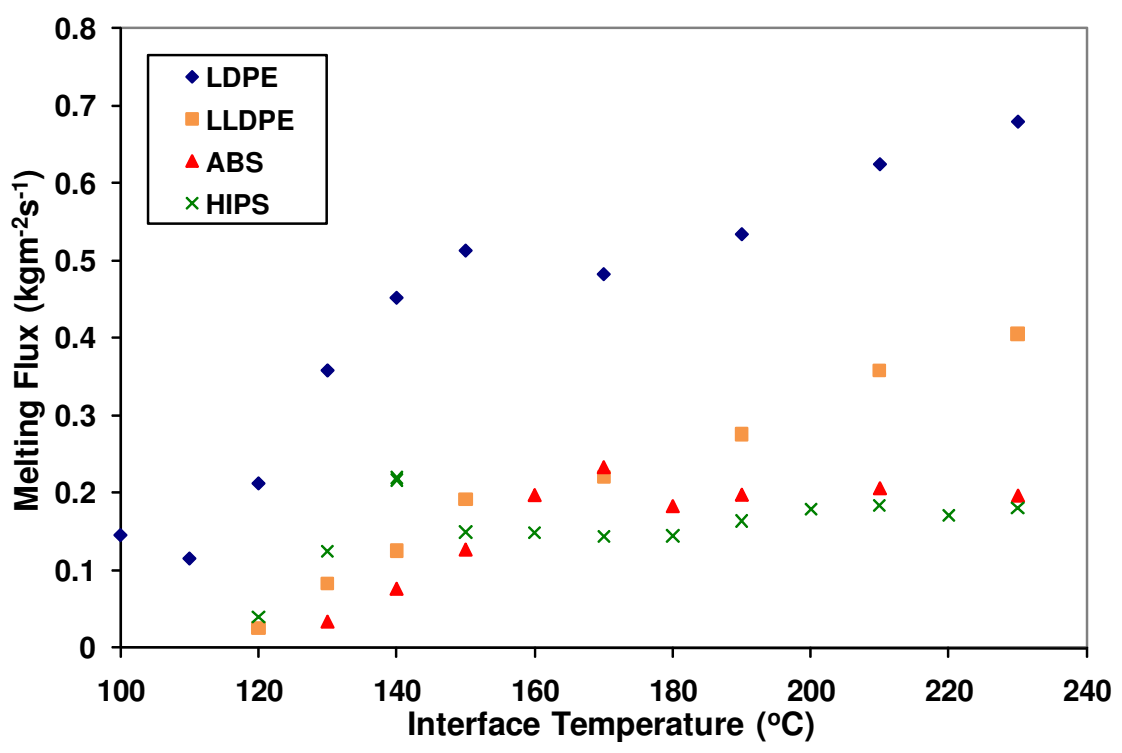

Figure 2.18: Melting flux for the four resins as a function of temperature at $61 \mathrm{~cm} / \mathrm{s}$.

was almost twice of the melting flux for the LLDPE resin. For the extrusion process, the reduced melting flux for the LLDPE resin will require a longer melting section in the screw than that required for the LDPE resin. This relationship of melting flux to commercial extrusion will be discussed later. The behavior of melting flux for the LDPE and LLDPE resins were different, however, for lower velocities. For instance, the melting fluxes were 0.112 and $0.222 \mathrm{~kg} /\left(\mathrm{m}^{2} \mathrm{~s}\right)$ for LDPE and LLDPE resins, respectively, at $7.6 \mathrm{~cm} / \mathrm{s}$ and 210 ${ }^{\circ} \mathrm{C}$. However, in the extrusion process, primary melting occurs between the solids bed and the barrel wall where the velocity gradient is relatively high, and the melting flux at very low velocities such as $7.6 \mathrm{~cm} / \mathrm{s}$ is not important for commercial extruders.

Relations to the Extrusion Process: Shear stresses resulting from either a frictional mechanism or viscous mechanism have an important role on the solids conveying and melting processes in plasticating single-screw extruders. Conveying of solid polymer in a single-screw extruder is optimized when the forces at the barrel-polymer interface and at the pushing flight, which advance the polymer in the screw channel, are maximized, and the retarding forces at the screw root and the trailing side of the screw flight are 
minimized [71]. For typical solids conveying processes, the barrel temperature is higher than the temperature of the screw, and the velocity of the solid bed relative to the barrel wall is considerably higher than that relative to the screw surface. For example, a typical $63.5 \mathrm{~mm}$ diameter extruder operating at a screw speed of $120 \mathrm{rpm}$ would have a solid bed velocity with respect to screw root and the barrel wall at about 8 and $40 \mathrm{~cm} / \mathrm{s}$, respectively, which are determined from the mass balance and the main circumferential velocity component, respectively. Thus, these different temperatures as well as the sliding velocities on barrel and screw surfaces can affect the forces that occur on the solid bed in the extruder channel as shown by the results in Figs. 2.10 through 2.13.

In order to maximize the solids conveying forces for the ABS and HIPS resins, the data presented in Figs. 2.12 and Fig. 2.13 show that the inside barrel surface temperature in the feed section should be about 160 and $130{ }^{\circ} \mathrm{C}$, respectively. At these temperatures the stresses at the interface are a maximum and thus will provide maximum forwarding forces at the barrel wall. In practice, for the ABS resin, the set barrel temperature for the solid conveying region of a single-screw extruder may need to be as high as $210{ }^{\circ} \mathrm{C}$ to maintain an inside barrel surface temperature near $160^{\circ} \mathrm{C}$. For the ABS as well as the HIPS resin, the temperature of the screw in the solids conveying region should be below $90{ }^{\circ} \mathrm{C}$. The screw temperature of less than $90{ }^{\circ} \mathrm{C}$ will create lower stresses, and thus, minimize the retarding forces at the screw root and trailing flight edge. For screws with diameters greater than about $150 \mathrm{~mm}$, cooling of the shank of the screw using water and a rotary union may be required to maintain optimal solids conveying. A further decrease in the screw temperature for the $A B S$ resin may result in an increase in the shear stress and the retarding forces, causing reduced solids conveying. Moreover, if the screw temperature increases to temperatures where the shear stress and the retarding forces are a maximum for the ABS and HIPS resins, solids conveying will be reduced, causing the downstream channels to become starved and leading to flow surging [69]. 
Previous research has shown that HIPS resins typically extrude at specific rates (rate/screw speed) less than those for ABS resins on the same extruder [72]. Since the melt densities for these resins are essentially the same at the processing temperatures [70], the higher specific rate for the ABS resins was attributed to higher solids conveying rate [72] which produces a higher pressure at the entry to the metering section. As previously discussed and as shown in Figs. 2.12 and 2.13, the ABS resin is capable of producing slightly higher forwarding forces and considerably lower retarding forces for solids conveying as compared to the HIPS resin. The higher forwarding and lower retarding forces for the ABS resin increases solids conveying rate, and thus, the downstream channel pressure. The higher downstream channel pressure is in part responsible for the higher specific rate for the ABS resin. To increase the specific rate for the HIPS resin, screw designers often increase the channel depth of the screw in the solids conveying section. The channel depth in the solids conveying section that is acceptable for HIPS resins often conveys $A B S$ resins at specific rates that are too high for the melting section of the screw, causing solid fragments in the metering section, which may be discharged with the extrudate. These fragments if large enough can cause a blockage towards the end of the metering section in a single-screw extruder that eventually lead to flow surging [73].

For the LDPE and LLDPE resins, solids conveying occurs due to the differences in the sliding velocity at the surfaces of the screw and barrel wall. As previously stated, the sliding velocities at the screw and barrel walls for the $63.5 \mathrm{~mm}$ diameter extruder are about 8 and $40 \mathrm{~cm} / \mathrm{s}$, respectively at a screw speed of $120 \mathrm{rpm}$. In an extruder operating at 90 and $130{ }^{\circ} \mathrm{C}$ at the screw and barrel surfaces, respectively, for LDPE resin the stress at the interfaces would be $0.24 \mathrm{MPa}$ (screw) and $0.27 \mathrm{MPa}$ (barrel) as shown in Fig. 2.10. This difference in shear stress is enough to properly convey LDPE resin.

The melting fluxes were measured at a pressure of $0.7 \mathrm{MPa}$ in order to compare the melting performances of the materials. For example, the melting flux for LLDPE resin was 
almost half of that for the LDPE resin at a roll surface velocity of $61 \mathrm{~cm} / \mathrm{s}$ and at a roll temperature of $210{ }^{\circ} \mathrm{C}$. This indicates that the melting length in an extruder needed to melt the LLDPE resin is about twice as long as that required for the LDPE resin. Thus, the same screw would not be optimal for the extrusion of these resins. According to the known processing attributes, LDPE resins can be extruded with screws having a relatively short melting section whereas LLDPE resins are typically extruded using screws with a relatively long melting section to completely melt the resin under stable conditions [74]. Thus, the melting data presented for the LDPE and LLDPE resins are consistent with known processing attributes. The effect of pressure on melting flux was not investigated in this study. However, the melting flux in Screw Simulator increases with increasing pressure as previously reported [8]. Similar increase in the melting flux at higher pressures was observed in extrusion processes by Klein [75].

\section{PC Resins with Different Melt Flow Rates (MFR)}

The results for $\mathrm{PC}$ resins were published earlier in one of our papers [2]. The shear stresses at the polymer-metal interface are shown by Figs. $2.19,2.20$, and 2.21 for the PC-3, PC-6 and $\mathrm{PC}-22$ resins, respectively. For all resins, two local maxima in the shear stress curves were observed; one was near the glass transition temperature at about $150{ }^{\circ} \mathrm{C}$, and the other at about $240{ }^{\circ} \mathrm{C}$. It is interesting to note that this kind of shear stress behavior was not observed for other amorphous materials such as acrylonitrile butadiene styrene (ABS) terpolymer and high-impact polystyrene (HIPS) resin [3, 8, 53, 67-70]. Two stress maxima, however, are apparent in the PC resin data measured by Mount and Chung [39].

For the PC-3 resin, the shear stress was nearly constant up to a temperature of about $100{ }^{\circ} \mathrm{C}$, and then it increased with increasing temperature to a maximum value at about $150{ }^{\circ} \mathrm{C}$ (at the $\mathrm{T}_{\mathrm{g}}$ ), as indicated by Fig. 2.19. Between temperatures of $150{ }^{\circ} \mathrm{C}$ and about $210{ }^{\circ} \mathrm{C}$, the shear stress decreased with increasing temperature. A second maximum in 


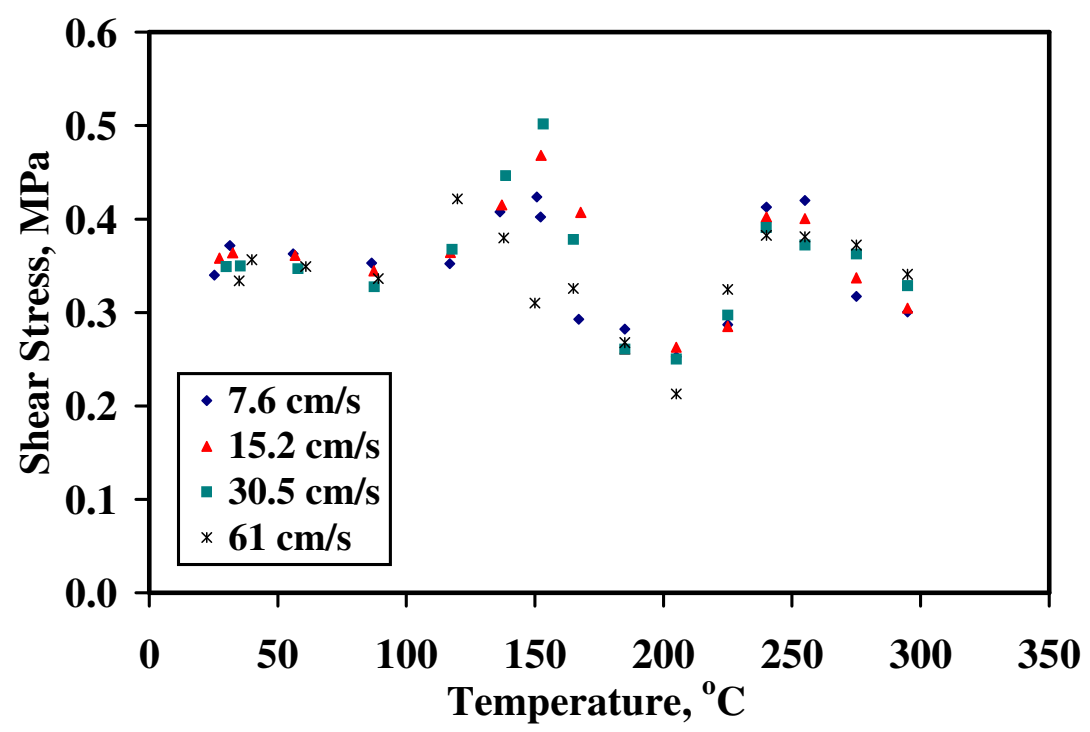

Figure 2.19: Shear stress at the interface for $\mathrm{PC}-3$ resin at $0.7 \mathrm{MPa}$ as a function of velocity and temperature.

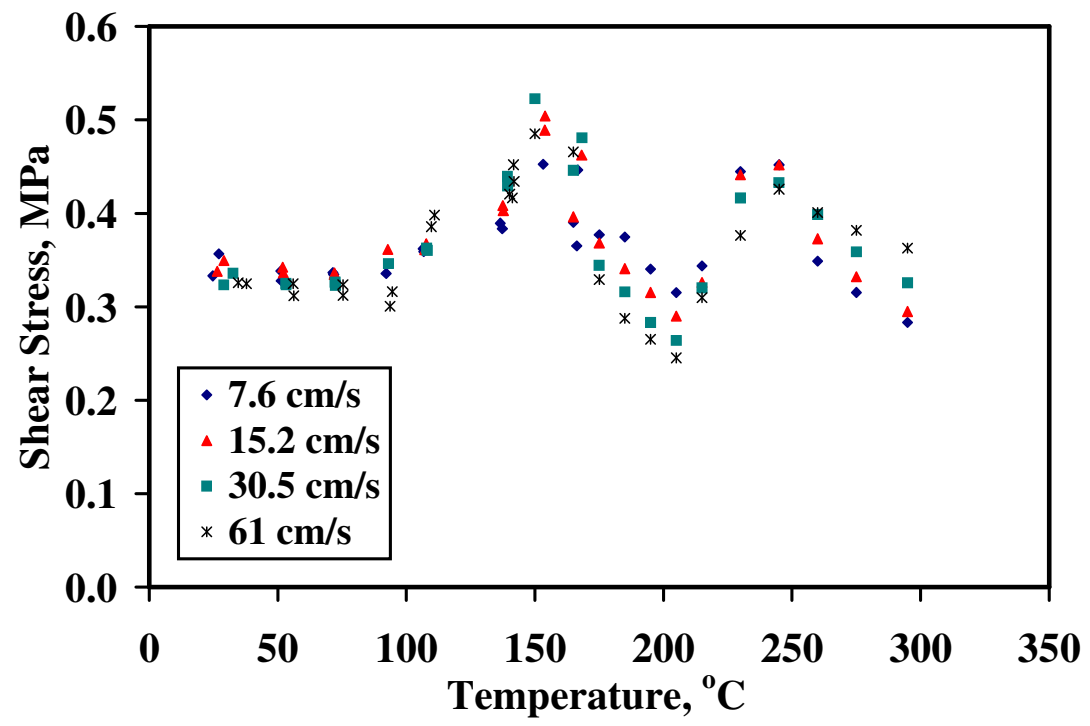

Figure 2.20: Shear stress at the interface for PC-6 resin at $0.7 \mathrm{MPa}$ as a function of velocity and temperature. 


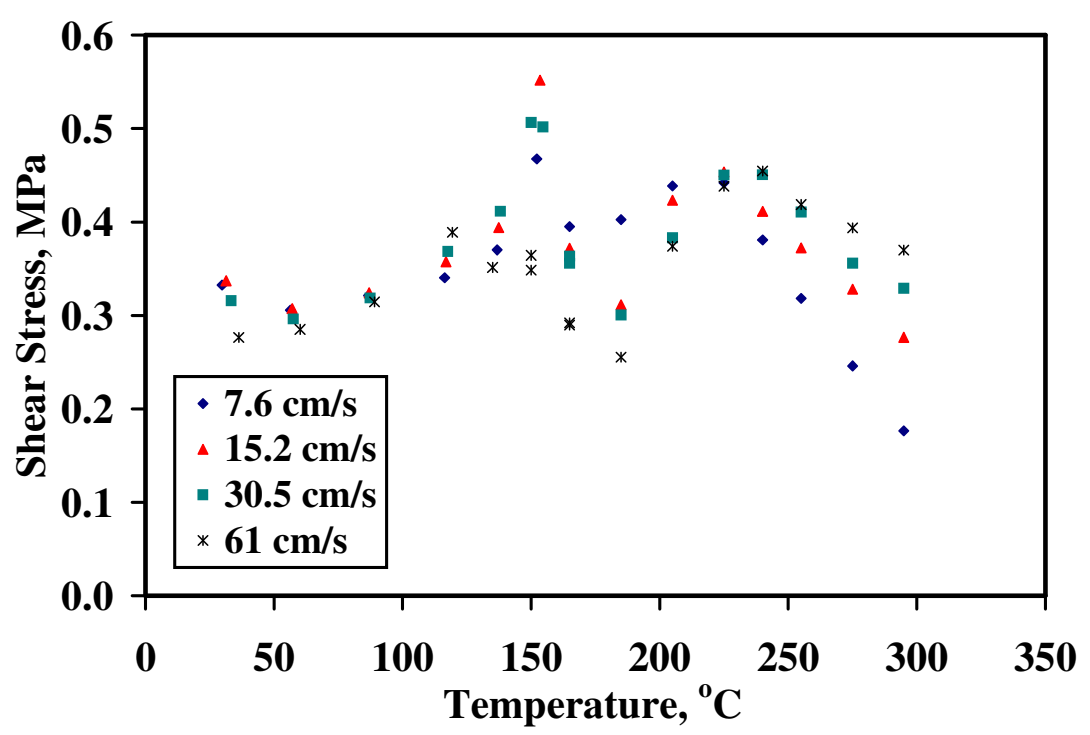

Figure 2.21: Shear stress at the interface for $\mathrm{PC}-22$ resin at $0.7 \mathrm{MPa}$ as a function of velocity and temperature.

the stress occurred at a temperature of about $240{ }^{\circ} \mathrm{C}$, and as the temperature increased further, the shear stress decreased. At temperatures higher than $275^{\circ} \mathrm{C}$ a polymer melt film was observed on the roll surface, and the shear stress increased with increasing velocity.

The shear stresses for the PC- 6 and PC-22 resins showed similar behavior as the PC-3 resin, as shown by Figs. 2.20 and 2.21. The main difference between the three PC resins was the stress response at temperatures higher than $260{ }^{\circ} \mathrm{C}$. As indicated in Figs. 2.19, 2.20, and 2.21, the stress was more dependent upon the sliding velocity for the PC-22 resin as compared to the PC-3 resin in this temperature range, and the PC- 6 resin had intermediate behavior. This result was due to the shear viscosity and its dependence on shear rate. For example, the shear viscosity of the PC-3 resin was moderately dependent on shear rate. As the velocity of the roll increased for this resin, the shear stress (viscosity times shear rate) did not change greatly. But for the more Newtonian PC-22 resin, the 


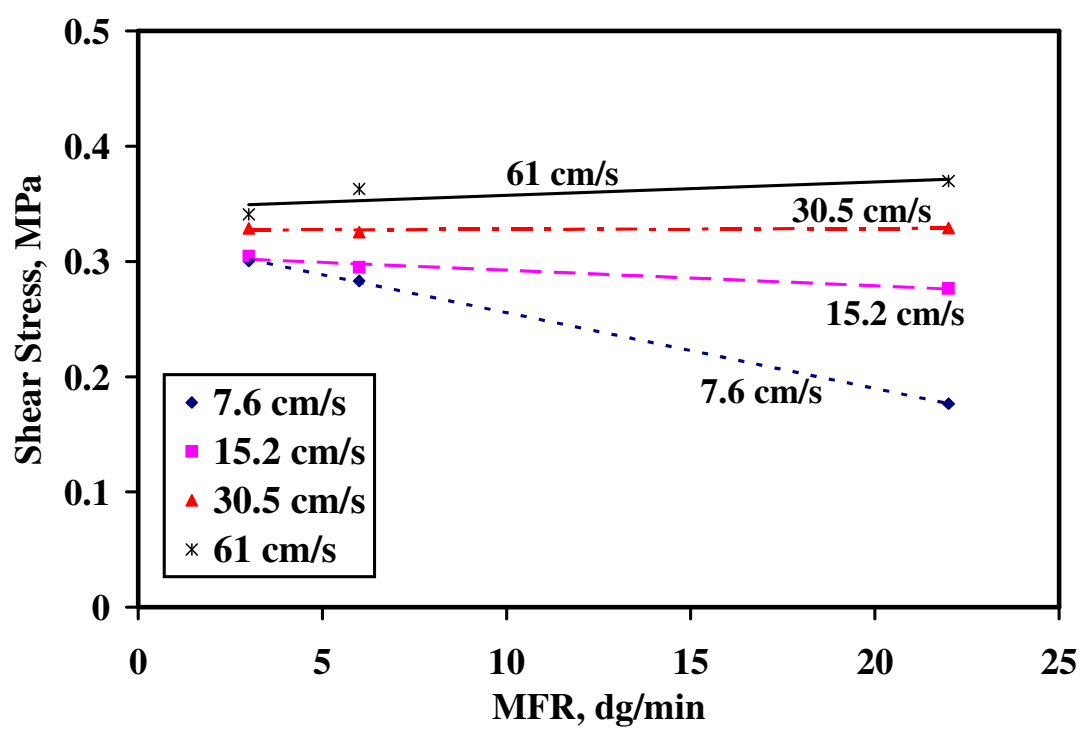

Figure 2.22: Shear stress as a function of velocity and MFR at $295{ }^{\circ} \mathrm{C}$.

shear stress was nearly linear with changing roll velocity. The shear viscosities of the PC resins can be found in Section 2.3.1. It is also interesting to note that as shown by Fig. 2.22, the shear stress did not decrease with increasing MFR for three PC resins in all velocities. For instance, at a velocity of $61 \mathrm{~cm} / \mathrm{s}$ and at a temperature of $295{ }^{\circ} \mathrm{C}$, the shear stress at the interface for the PC-3 and PC-22 resins were nearly the same at 0.34 and 0.37 MPa, respectively. This result was slightly different than that reported by Hogan et al. [50]; that is, their results showed that the shear stress decreased with increasing MFR for resins with a wider MFR range.

The melting flux data for the three $\mathrm{PC}$ resins as a function of velocity and temperature are shown in Figs. 2.23, 2.24, and 2.25. The temperature where mechanical melting was first observed is different for the three $\mathrm{PC}$ resins - It is 165,165 , and $150{ }^{\circ} \mathrm{C}$ for the $\mathrm{PC}-3$, PC-6, and PC-22 resins, respectively. The mechanical melting temperature [8] is defined as the temperature where a significant portion of the thermal energy for melting originates from a mechanical energy dissipative process. A melt film on the roll surface, however, 


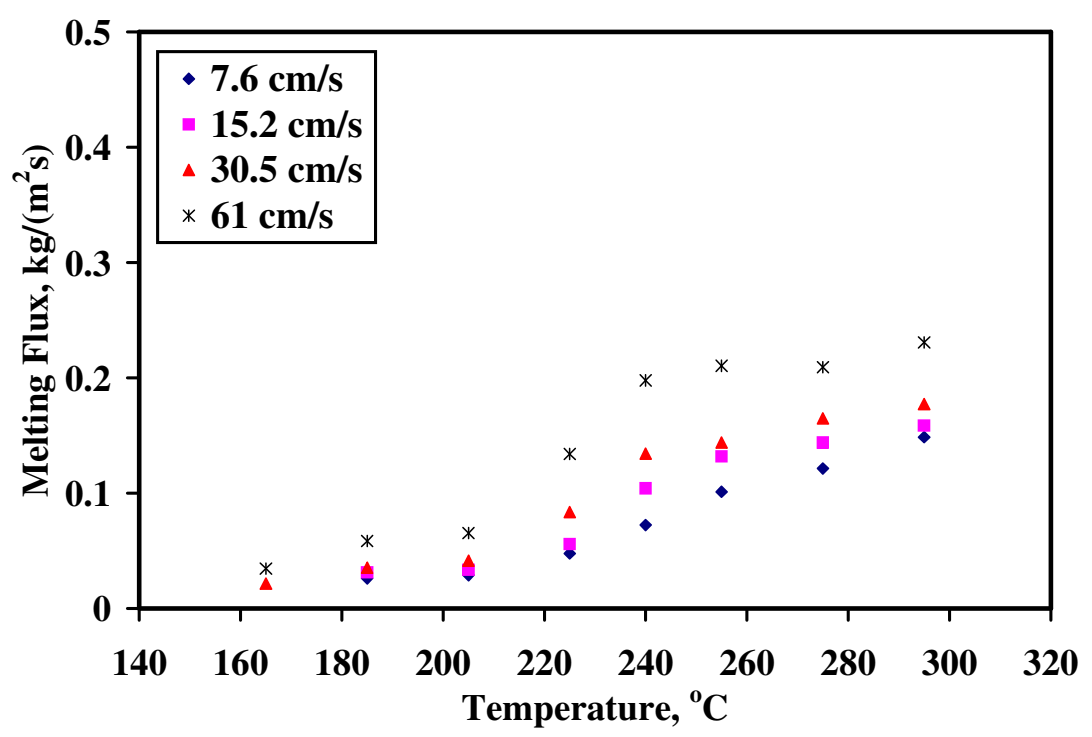

Figure 2.23: Melting flux for $\mathrm{PC}-3$ resin at $0.7 \mathrm{MPa}$ as a function of velocity and temperature.

was observed at significantly higher temperatures. For the PC-3 resin, the first melt film on the roll surface was observed at $275{ }^{\circ} \mathrm{C}$. As shown by these figures, the melting flux increased with increasing temperature and velocity. This result was expected and it has been observed before for other resins $[3,8,53,67-70]$. The melting flux also increased with increasing MFR, as shown by Fig. 2.26. For example, at a velocity of $61 \mathrm{~cm} / \mathrm{s}$ and at a temperature of $295{ }^{\circ} \mathrm{C}$, the melting fluxes for the PC-3, PC-6, and PC-22 resins were measured as $0.23,0.28$, and $0.45 \mathrm{~kg} /\left(\mathrm{m}^{2} \mathrm{~s}\right)$, respectively. This melting behavior with flow index has been observed with other resins [49] and with PC resins [50].

It is known that the role of the shear stress at a polymer-metal interface on solids conveying and melting processes is very important. It is shown in Figs. 2.19 through 2.21 that the shear stress has two local maxima with increasing temperature for all three PC resins. These two peaks were observed in the data by Mount and Chung [39]. Their lack of experimental data for temperatures less than $150{ }^{\circ} \mathrm{C}$, however, obscures the first 


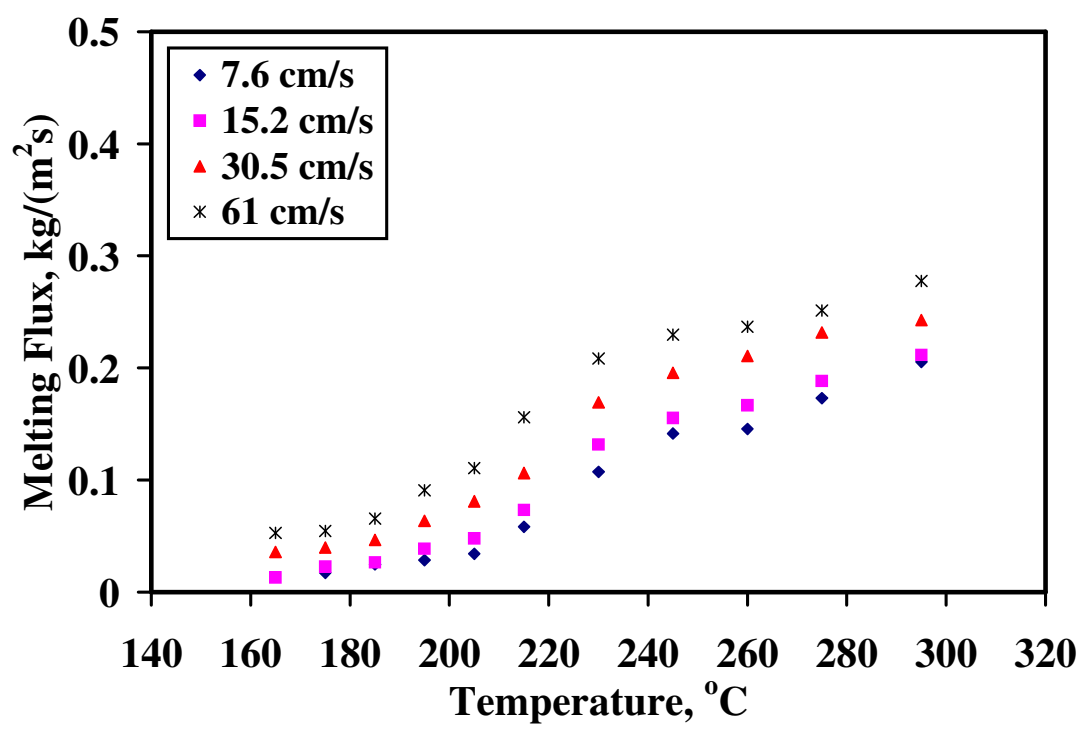

Figure 2.24: Melting flux for PC-6 resin at $0.7 \mathrm{MPa}$ as a function of velocity and temperature.

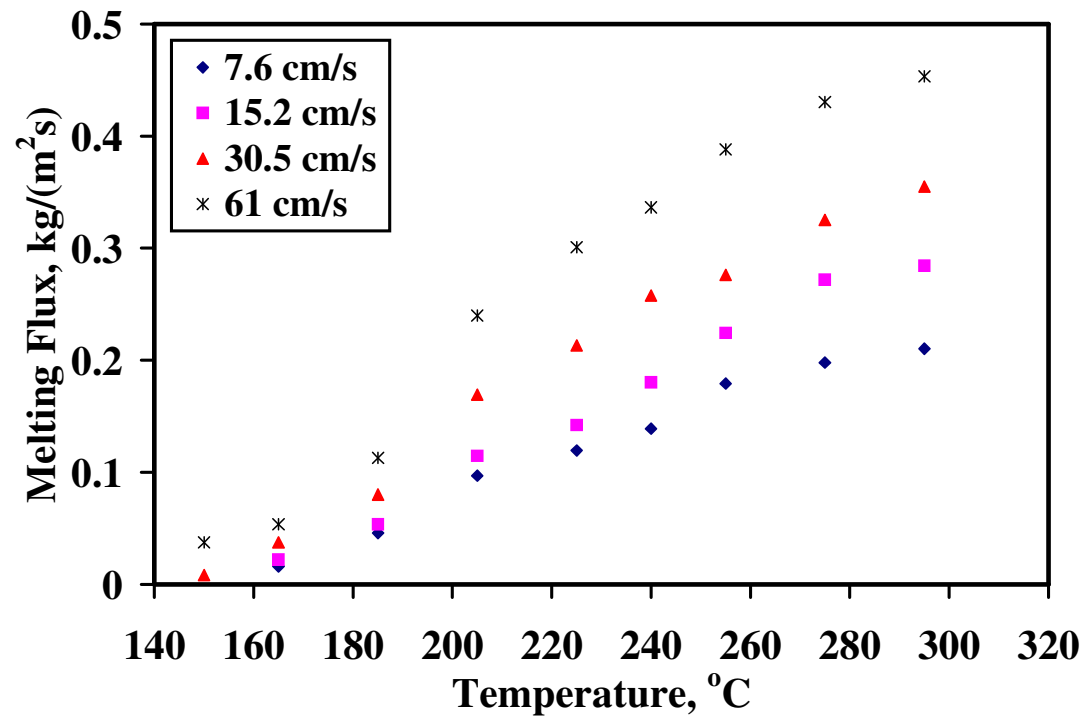

Figure 2.25: Melting flux for PC-22 resin at $0.7 \mathrm{MPa}$ as a function of velocity and temperature. 


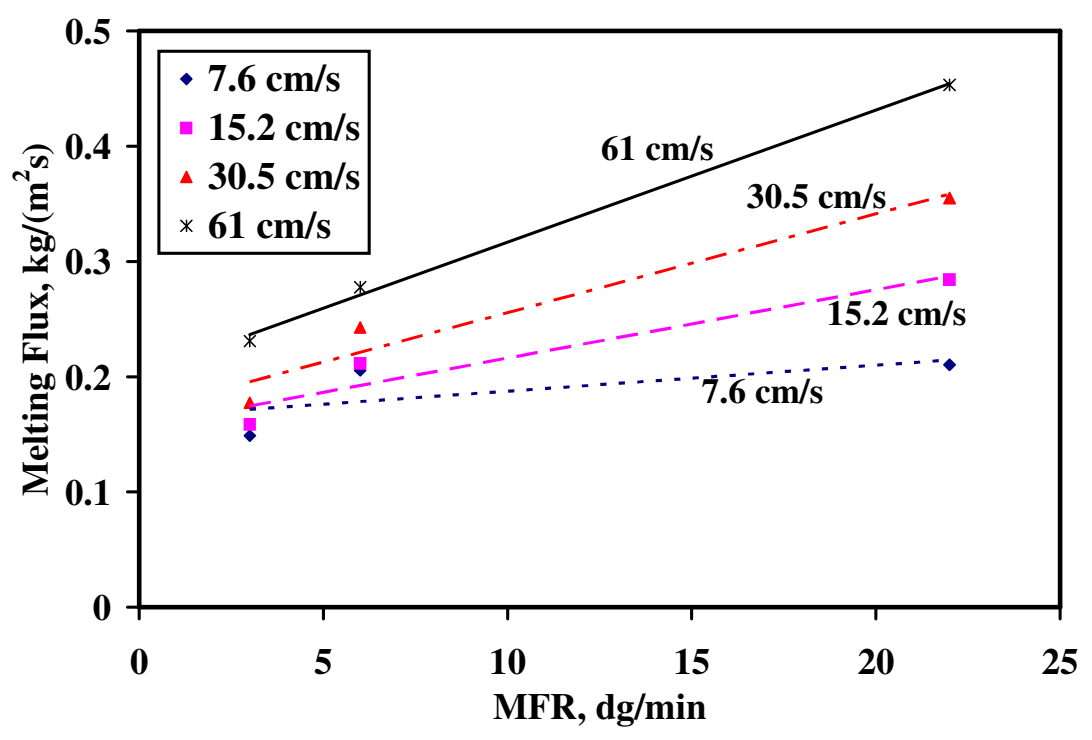

Figure 2.26: Melting flux as a function of velocity and MFR at $295^{\circ} \mathrm{C}$.

maximum. This behavior of the shear stress has an important role on solids conveying for the extrusion process which will be discussed for a commercial extruder later in this section.

The melting fluxes for three PC resins were also measured to compare the melting performances of the materials. The results showed an increase in melting flux with increasing MFR. These results were consistent with the previous work for PC resin [50].

Case Study: The shear stress data were used to explain a flow surging problem for a commercial extruder. For this case, a medium diameter extruder was running the PC-6 resin. The extruder was a plasticating, two-stage, vented machine. A gear pump was positioned between the extruder and the die. The schematic of the extruder is given in Fig. 2.27. The screw speed was controlled such that the inlet pressure to the pump (or extruder discharge pressure) was $9 \mathrm{MPa}$. During certain time periods, the extruder would flow surge and limit the production capacity of the line. After numerous hours of troubleshooting, the root cause of the surging was determined to be a high temperature 


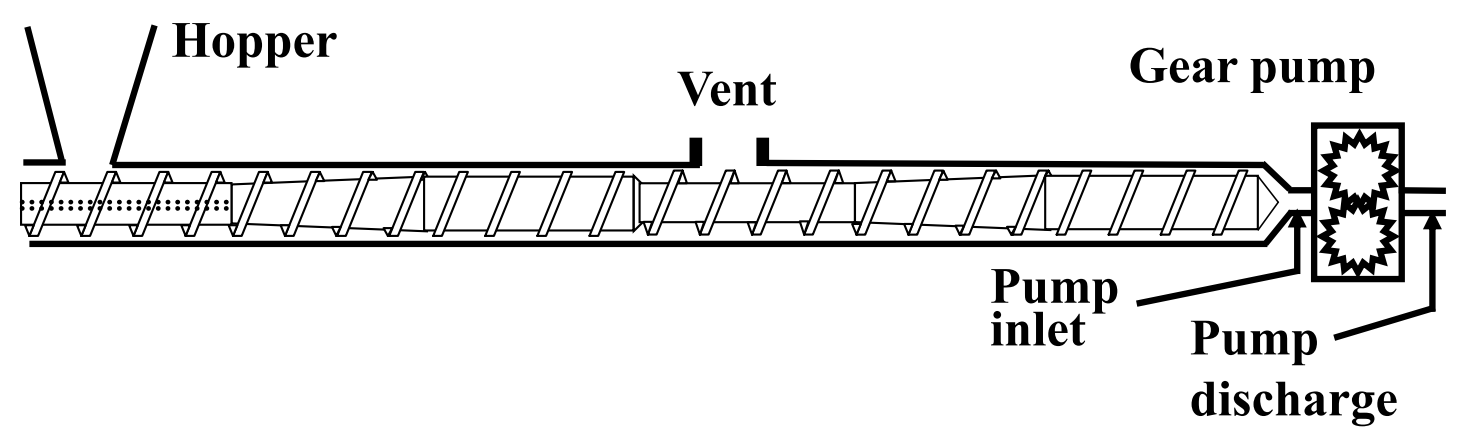

Figure 2.27: Schematic of the extruder used in the case study of PC-6 resin.

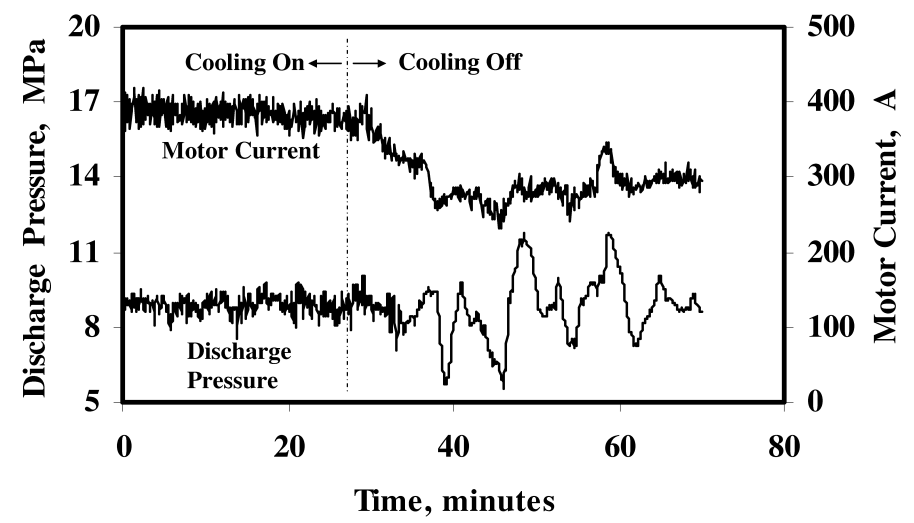

Figure 2.28: Discharge pressure and motor current for an extruder running PC-6 resin with and without cooling water to the root of the screw.

on the screw in the solids conveying section. The root cause of the flow surging is clearly shown by Figs. 2.28 and 2.29. The extruder operated stably while a high level of cooling water was flowed to the shank of the screw for the first 28 minutes of these figures. At 28 minutes, the cooling water was turned off, causing the solids conveying section of the screw to increase in temperature and the extruder to flow surge. As shown by these figures, the motor current, screw speed, and discharge pressure became very unstable. Although not the focus of this work, the behavior of the process during the surging was expected [69]. High levels of cooling water to the shank of the screw were used to maintain a stable operation. 


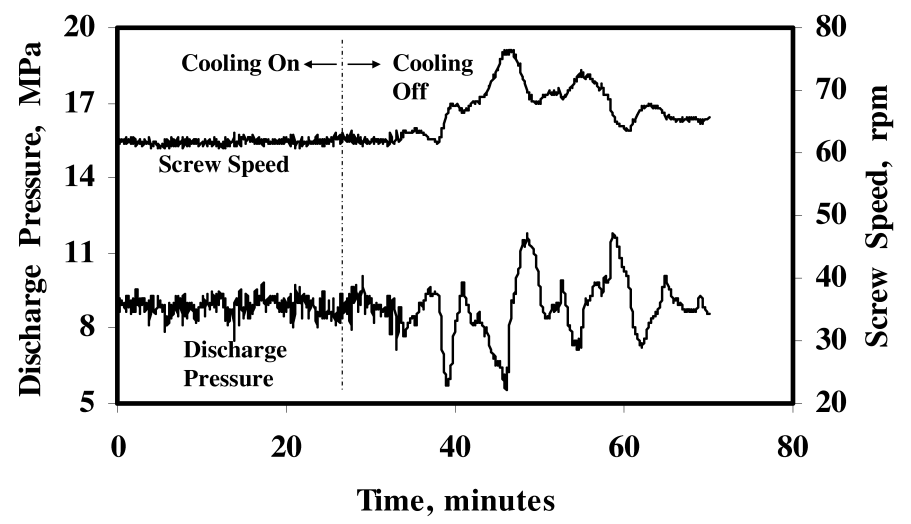

Figure 2.29: Discharge pressure and screw speed for an extruder running PC-6 resin with and without cooling water to the root of the screw.

As mentioned before, optimal solids conveying occurs when the advancing forces at the barrel wall and pushing flight are maximized and the retarding forces at the screw surface are minimized [71]. These forces depend on the geometry of the channel and are directly proportional to the coefficient of dynamic friction for temperatures less than the melting or devitrification temperature and on viscous forces (or stresses) for higher temperatures. The coefficient of friction is related to the stress at the interface as in Equation 2.5. Since the coefficient of dynamic friction and stress at the interface depend on temperature, pressure, and velocity, surface temperature changes for the barrel and screw in the feeding section will strongly affect the performance of the extruder. If the surface temperatures become too different from the optimal values, flow surging and loss of rate will occur. In order to maximize the forwarding force and thus shear stress at the barrel surface for the PC-6 resin, the inside barrel wall temperature in the feed section should be about $240{ }^{\circ} \mathrm{C}$, as shown by Fig. 2.20. In practice, an axial temperature gradient will exist between the water cooled feed casing and the first heated zone of the extruder barrel, and temperature sensors are generally not capable of measuring the inside surface temperature of the barrel accurately. Thus, an experimentally determined first zone temperature in the range of 290 to $320^{\circ} \mathrm{C}$ and a feed casing temperature around 35 
to $45^{\circ} \mathrm{C}$ are acceptable. For the screw, both forwarding and retarding forces occur, yet the temperature of the screw surfaces must be controlled to the same temperature. Based on the data here, the optimal screw temperatures for $\mathrm{PC}$ resins are those less than about $120{ }^{\circ} \mathrm{C}$. The extrusion data presented here are consistent with known extrusion behavior and the stress at the interface data of Fig. 2.20.

\section{Discussion on Shear Stress and Melting Flux Results for PC Resins with Different} Melt Flow Rates (MFR): As shown in Figs. 2.22 and 2.26, at a temperature of 295 ${ }^{\circ} \mathrm{C}$, an increase in MFR resulted in a decrease in shear stress and almost no change in melting flux at a velocity of $7.6 \mathrm{~cm} / \mathrm{s}$ whereas for a velocity of $61 \mathrm{~cm} / \mathrm{s}$, the shear stress was almost constant and the melting flux was increasing with an increase in MFR. At temperatures above the melting or devitrification temperature, the shear stress is defined as $\tau=\eta \dot{\gamma}$ and the melting flux is proportional to $\eta \dot{\gamma}^{2}$ where $\eta$ is the shear viscosity and $\dot{\gamma}$ is the shear rate. With these definitions of the shear stress and the melting flux, only a decrease in shear stress and an increase in shear rate with increasing MFR supports the experimental observations at a velocity of 7.6 and $61 \mathrm{~cm} / \mathrm{s}$ discussed above. Thus, as the MFR increases, $\eta$ should decrease and $\dot{\gamma}$ should increase in order to be consistent with the experimental data. As shown in Fig. 2.36, the shear viscosity of the PC resin at the same shear rate decreases with an increase in MFR. In order $\dot{\gamma}$ to increase with increasing MFR, the ratio of velocity to average melt film thickness should increase. Since the velocity at the surface of the rotating barrel was set to a constant, assuming a linear velocity profile along the melt film, the average melt film thickness should decrease in order to result in an increase in shear rate. Thus, as the MFR increases, the average melt film thickness should decrease, which is counter-intuitive. It should be noted here that the shear viscosity is also a function of shear rate, but the effect of shear rate on shear viscosity of PC resins is smaller compared to that of MFR as shown in Fig. 2.36. 


\subsection{Material Characterization Experiments}

The values of the various material properties are needed to simulate the melting and flow of polymer in a single-screw extruder. In particular, the values for thermal conductivity, heat capacity, density and the viscosity of the polymer in solid as well as liquid state are needed. In simulations, the values of thermal conductivity, heat capacity and density of polymer were assumed to be constant for both solid and liquid states. However, since a change in temperature or shear rate results in a significant change in viscosity, the dependency of the viscosity on shear rate and temperature was determined. A DMS (Dynamic Mechanical Spectrometer) was used to capture the shear rate and temperature dependency of shear viscosity for ABS, LDPE and PC resins. The DMS used for the experiments comprised two parallel plates. The bottom plate had a cylindrical shape whereas the top plate was in conical shape. The cylindrical plate at the bottom was stationary and the conical plate at the top was oscillating at different frequencies. Since the top plate had a conical shape, the shear rate was constant everywhere in between the plates. The torque needed to rotate the top plate and the rate of angular rotation (rotational speed) were measured at various temperatures. The rate of angular rotation and torque data were then used to calculate the shear rate and stress on the plates. Last, the shear viscosity was calculated as the ratio of the stress and shear rate. The results were used to generate the shear viscosity versus shear rate plots at various temperatures. The experimental data of shear viscosity at specific shear rates and temperatures were also used to fit the viscosity model parameters that were needed for the numerical simulations. It should be mentioned here that the DMS used for the experiments was designed to accurately measure lower shear rates; i.e. shear rates up to $100 \mathrm{~s}^{-1}$. Experimental data at higher shear rates using a capillary rheometer may improve the accuracy of the viscosity model at higher shear rates. 


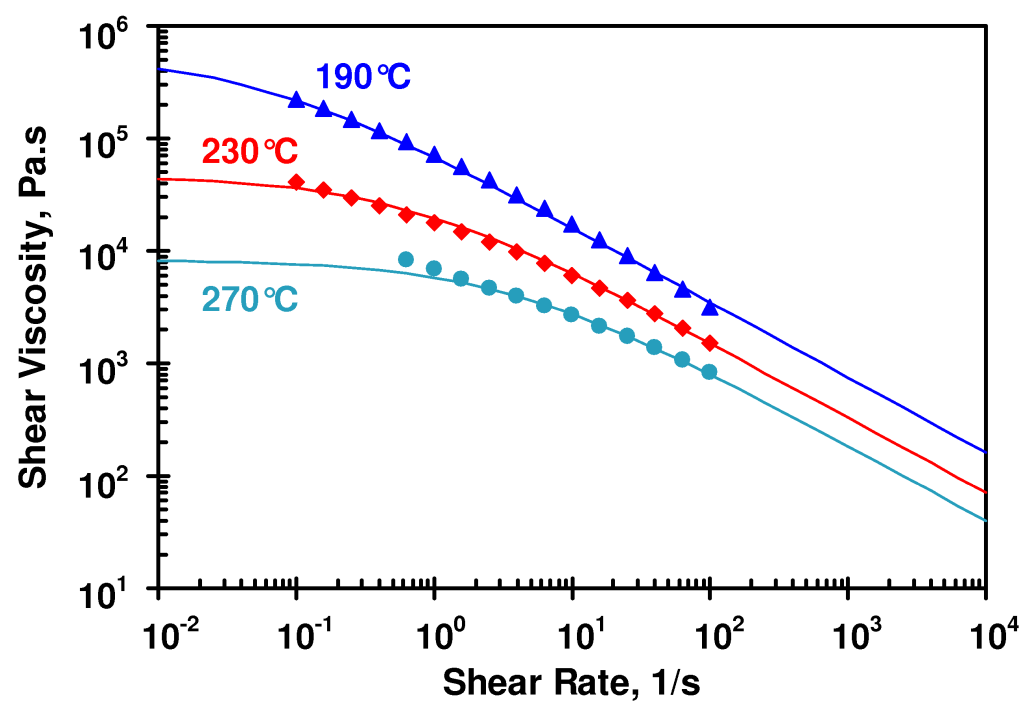

Figure 2.30: Shear viscosity data (symbols) and Cross-WLF model fit (curves) to the viscosity data for the $A B S$ resin at 190,230 , and $270{ }^{\circ} \mathrm{C}$.

\subsubsection{Experimental Results and Discussion}

The shear viscosities of the ABS, LDPE and PC resins measured at various temperatures are given in this section. The shear viscosity data for these resins were used to fit the Cross-WLF model which is described in Section 3.3. The shear viscosity data for the ABS resin at temperatures of 190,230 , and $270{ }^{\circ} \mathrm{C}$ is shown in Fig. 2.30 . The glass transition temperature of the $\mathrm{ABS}$ resin is around $100{ }^{\circ} \mathrm{C}$, and almost no flow occurs below $150{ }^{\circ} \mathrm{C}$. The zero-shear viscosity of the ABS resin predicted by the WLF model is shown in Fig. 2.31. It is evident from Fig. 2.31 that above $175{ }^{\circ} \mathrm{C}$ (liquid) the zero-shear viscosity is $10^{6}$ Pa.s, whereas the zero-shear viscosity below $125{ }^{\circ} \mathrm{C}$ (solid) is greater than $10^{9} \mathrm{~Pa}$.s. Furthermore, the viscosity of the liquid polymer at typical shear rate encountered in the melting section of a single-screw extruder will be much less than the zero-shear viscosity. Therefore, there is a large difference between the viscosity of the solid and liquid polymer.

The shear viscosity data for the LDPE resin measured at temperatures of 190, 230, and $240{ }^{\circ} \mathrm{C}$ is shown in Fig. 2.32. Since LDPE is a semi-crystalline polymer, the melting 


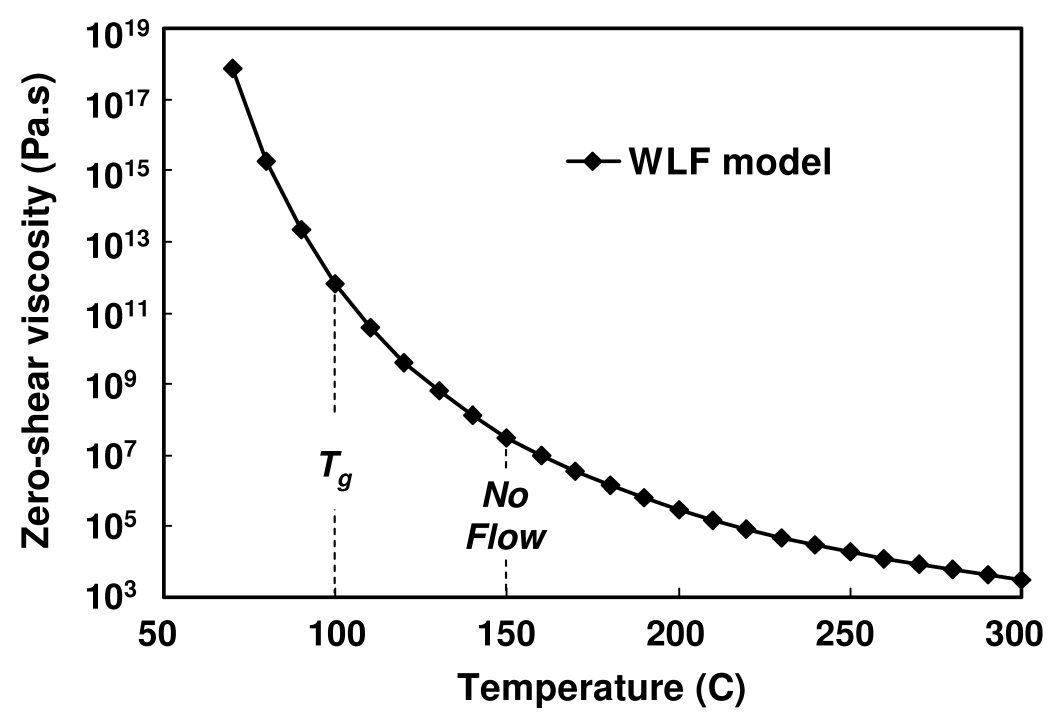

Figure 2.31: The change on zero-shear viscosity with respect to temperature for the ABS resin.

temperature is used to distinguish the solid and liquid polymer. If the temperature of the polymer is above the melting temperature, which is $111^{\circ} \mathrm{C}$, the polymer is assumed to be in liquid form. The WLF model was also used to predict the zero-shear viscosity of the LDPE resin.

The shear viscosities of the PC resins were measured at temperatures of 240,260 , 280 and $300{ }^{\circ} \mathrm{C}$. The results are shown in Figs. 2.33, 2.34, and 2.35 for PC-3, PC-6 and PC-22 resins, respectively. For comparison, the shear viscosities of these resins at $280{ }^{\circ} \mathrm{C}$ are also given in Fig. 2.36. It is clear from the Fig. 2.36 that in this range of shear rate (from 1 to $1000 \mathrm{~s}^{-1}$ ), PC-22 is more Newtonian than the other resins. That is, the shear viscosity of the $\mathrm{PC}-22$ resin decreased at a smaller rate with increasing shear rate. The glass transition temperatures of the $\mathrm{PC}$ resins were about $150{ }^{\circ} \mathrm{C}$.

As mentioned earlier in this section, the shear viscosity data for all resins were used to fit the Cross-WLF model. The values of Cross-WLF viscosity model parameters thus obtained are given in Table 2.4. The definitions of the Cross-WLF model parameters are given in Section 3.3. 


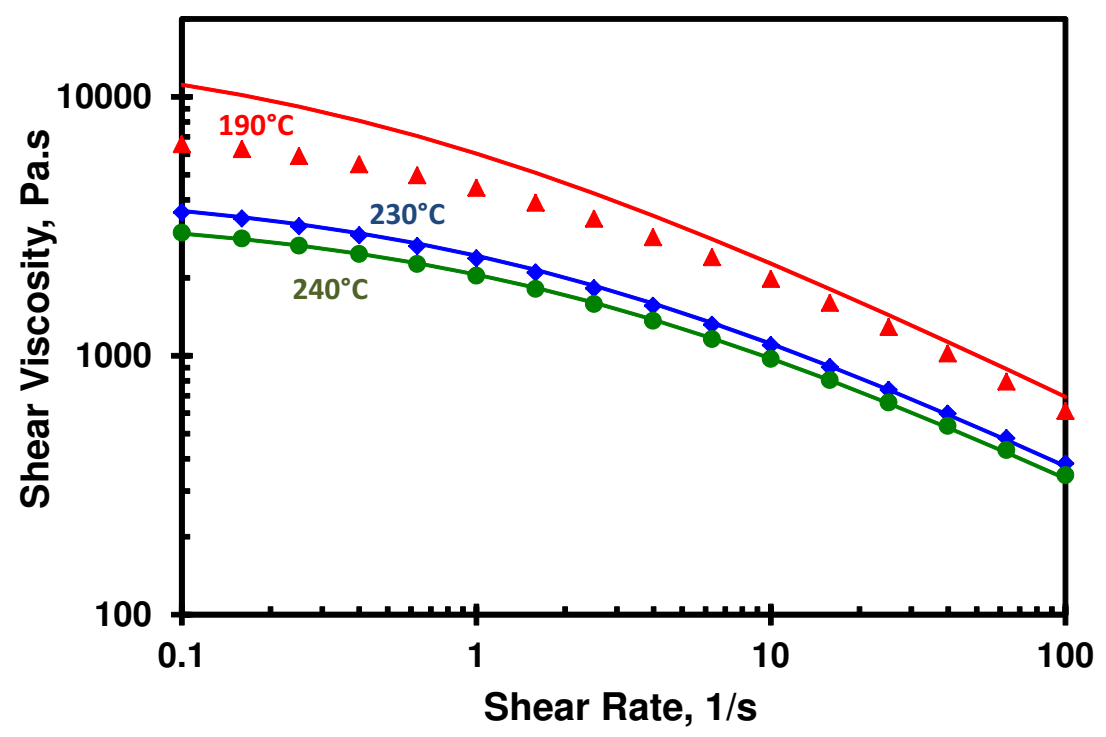

Figure 2.32: Shear viscosity data (symbols) and Cross-WLF model fit (curves) to the viscosity data for the LDPE resin at 190,230 , and $240{ }^{\circ} \mathrm{C}$.

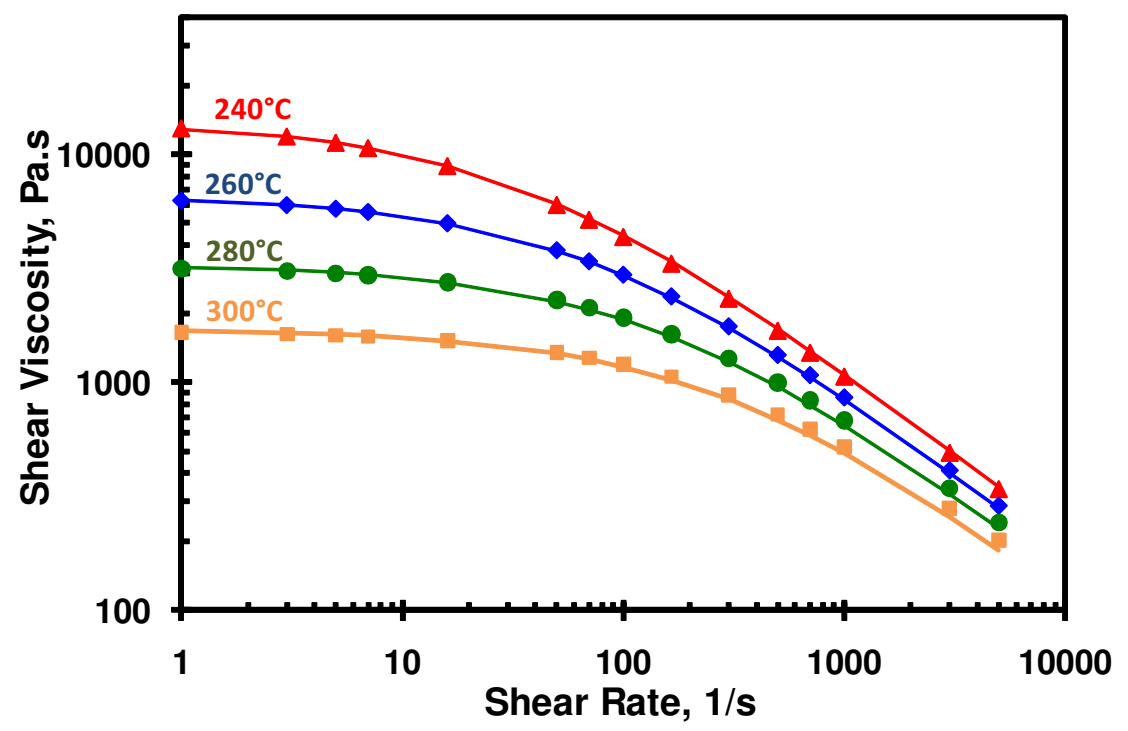

Figure 2.33: Shear viscosity data (symbols) and Cross-WLF model fit (curves) to the viscosity data for the $\mathrm{PC}-3$ resin at $240,260,280$, and $300{ }^{\circ} \mathrm{C}$. 


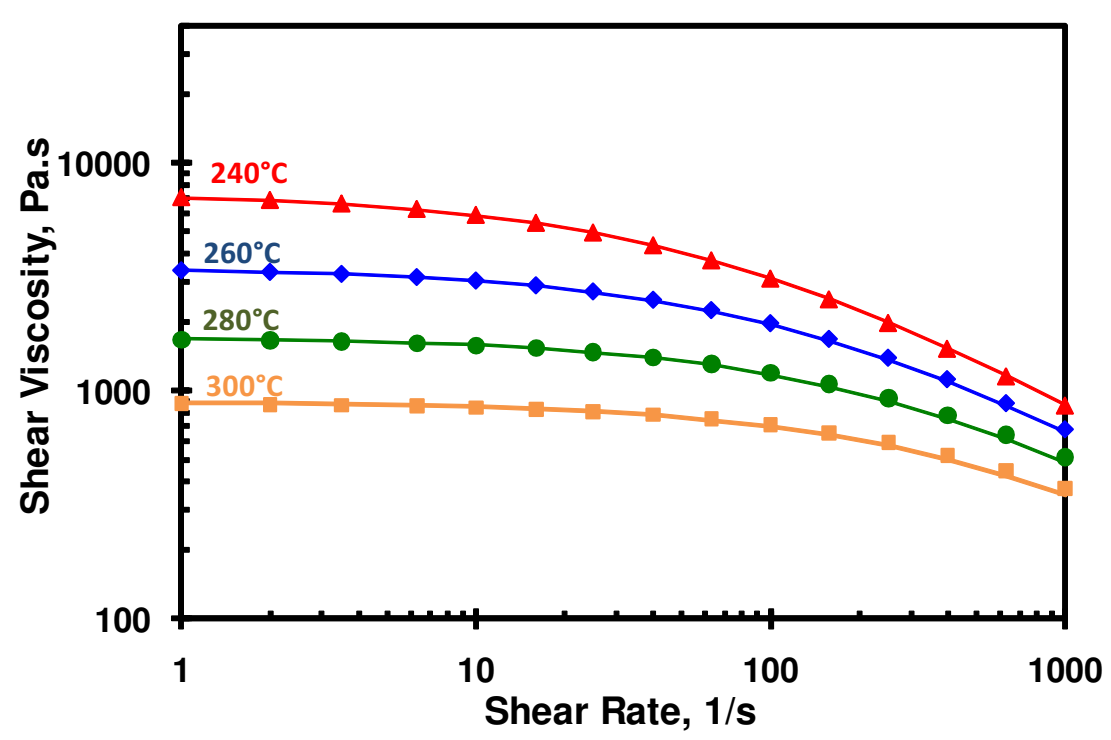

Figure 2.34: Shear viscosity data (symbols) and Cross-WLF model fit (curves) to the viscosity data for the PC- 6 resin at $240,260,280$, and $300{ }^{\circ} \mathrm{C}$.

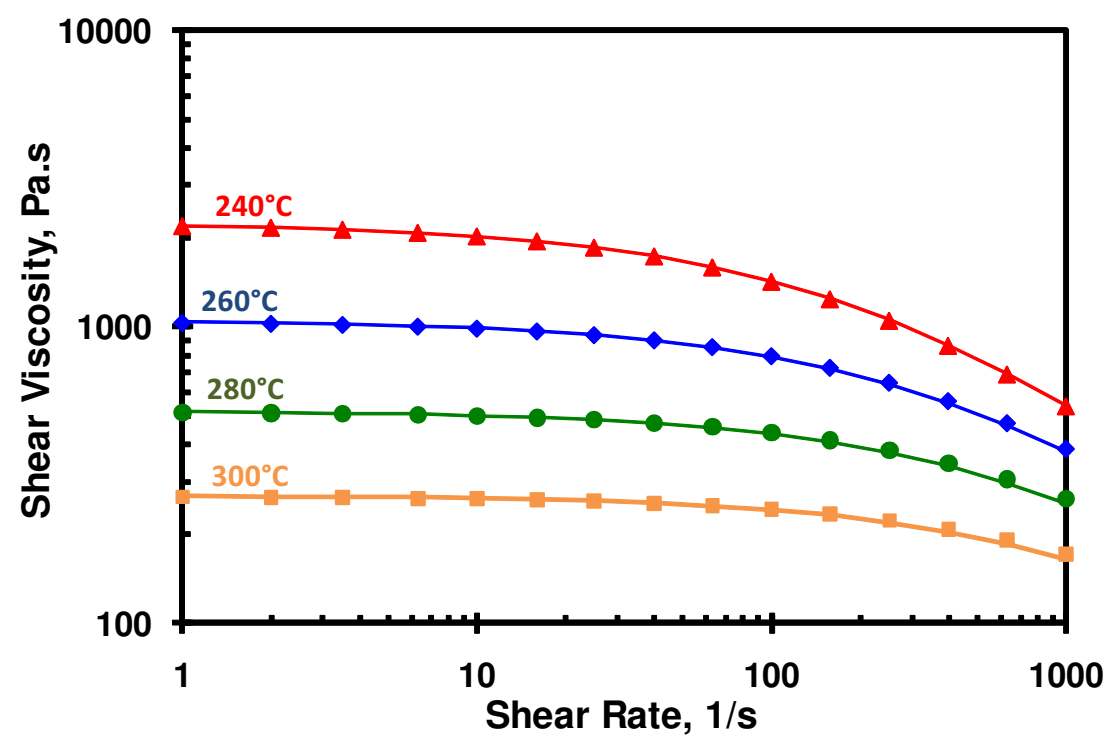

Figure 2.35: Shear viscosity data (symbols) and Cross-WLF model fit (curves) to the viscosity data for the $\mathrm{PC}-22$ resin at $240,260,280$, and $300{ }^{\circ} \mathrm{C}$. 


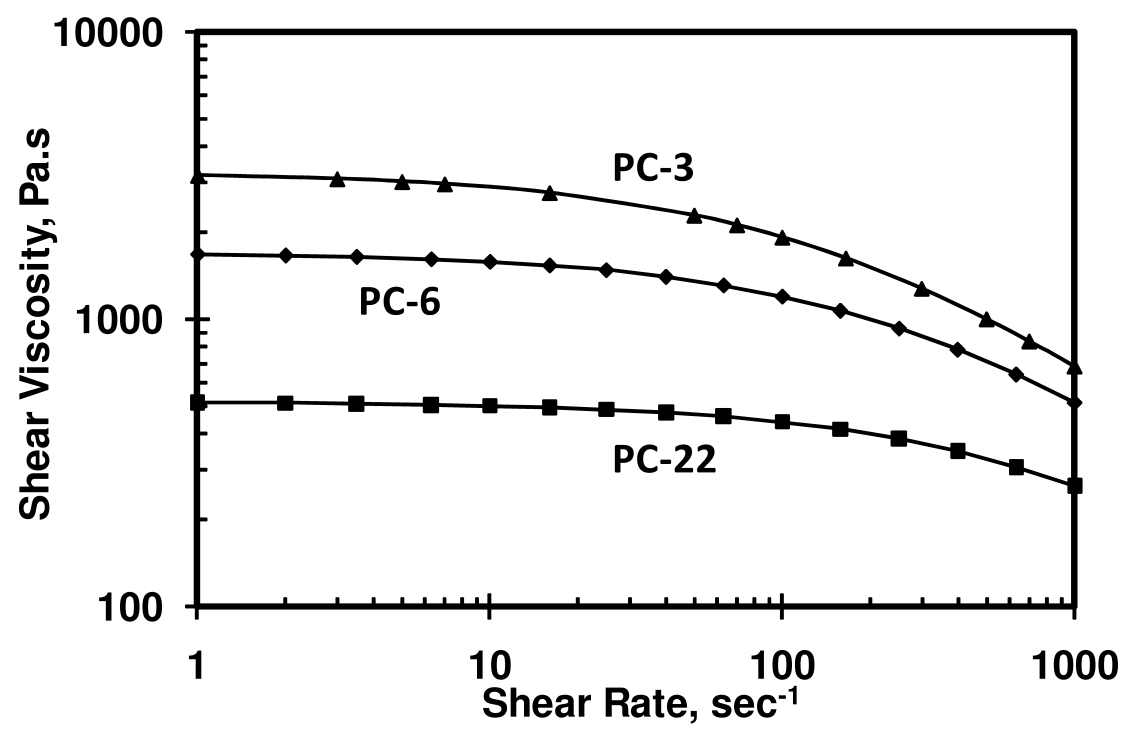

Figure 2.36: Shear viscosity for all PC resins at a temperature of $280{ }^{\circ} \mathrm{C}$.

Table 2.4: The values of the Cross-WLF viscosity model parameters for the ABS, LDPE, and $P C$ resins.

\begin{tabular}{|c|c|c|c|c|c|c|}
\hline \multirow[b]{2}{*}{ Resin } & \multicolumn{6}{|c|}{ Cross-WLF Model Parameters } \\
\hline & $\begin{array}{c}D_{1} \\
(\text { Pa.s })\end{array}$ & $A_{1}$ & $\begin{array}{l}A_{2} \\
(\mathrm{~K})\end{array}$ & $\begin{array}{c}\tau^{*} \\
(\mathrm{~Pa})\end{array}$ & $n$ & $\begin{array}{l}T_{a} \\
(\mathrm{~K})\end{array}$ \\
\hline ABS & $3.63 \times 10^{11}$ & 27.21 & 92.85 & $2.90 \times 10^{4}$ & 0.33 & 373 \\
\hline LDPE & $2.29 \times 10^{10}$ & 18.95 & 27.14 & $6.26 \times 10^{3}$ & 0.44 & 384 \\
\hline PC-3 & $8.85 \times 10^{5}$ & 25.85 & 470.07 & $4.93 \times 10^{5}$ & 0.26 & 423 \\
\hline PC-6 & $4.73 \times 10^{5}$ & 25.91 & 469.96 & $4.89 \times 10^{5}$ & 0.26 & 423 \\
\hline PC-22 & $1.47 \times 10^{5}$ & 25.72 & 462.03 & $4.86 \times 10^{5}$ & 0.26 & 423 \\
\hline
\end{tabular}


Table 2.5: Various material properties of the ABS, LDPE, and PC resins used in the numerical simulations.

\begin{tabular}{lccccc} 
& \multicolumn{5}{c}{ Resin } \\
\cline { 2 - 6 } & ABS & LDPE & PC-3 & PC-6 & PC-22 \\
\cline { 2 - 6 } Density $\left(\mathrm{g} / \mathrm{cm}^{3}\right)$ & 0.94 & 0.83 & 1.07 & 1.07 & 1.07 \\
$\begin{array}{l}\text { Heat capacity } \\
(\mathrm{J} / \mathrm{kg} . \mathrm{K})\end{array}$ & 2345 & 2470 & 1960 & 1960 & 1960 \\
$\begin{array}{l}\text { Thermal conductivity } \\
(\mathrm{W} / \mathrm{m} . \mathrm{K})\end{array}$ & 0.18 & 0.24 & 0.19 & 0.19 & 0.19 \\
\cline { 2 - 6 } & & & & &
\end{tabular}

It is understood that the values of density, heat capacity and thermal conductivity will change over the range of temperature observed in numerical simulations. However, the variation of these material properties with temperature was not known for the ABS. LDPE, and $P C$ resins used in this work. Therefore, in numerical simulations, the values of the polymer density, heat capacity and thermal conductivity were assumed to be constant. These constant values of density, heat capacity and thermal conductivity of the ABS, LDPE and PC resins are given in Table 2.5. 


\section{Chapter 3}

\section{Investigation of Polymer Melting and}

Flow in Single-screw Extruders:

\section{Numerical Methods, Results and}

\section{Discussion}

In this study, three-dimensional finite element simulations of the melting process in the compression section of the extruder were performed by solving the conservation equation for mass, momentum and energy along with a constitutive equation. Numerical methods used to accomplish the task of simulating the melting and flow of polymers in single-screw extruders are discussed in this chapter. First, the governing equations of mass, momentum and energy are described. Next, the finite element formulation of these equations are given. The description of the viscosity model and the basic information about the numerical simulations such as boundary conditions, finite element mesh properties etc. is given later followed by the implementation of the GPU code to reduce the computation time. In the end, the numerical results obtained using the numerical methods discussed above are presented. 


\subsection{Governing Equations}

For the finite element simulations of polymer melting in single-screw extruders presented in this dissertation, it was assumed that the polymer material is isotropic and it satisfies the continuum assumption. The isotropy is basically defined as the independence of material properties on direction. The assumption of continuum is typically described such that the material properties such as density, temperature, stress etc., in a medium cannot change abruptly, cannot become infinity and cannot include discontinuity at a single point. In general, the continuum assumption holds for macro-scale studies whereas it is avoided in micro-scale.

In this study, it was also assumed that the flow is steady and incompressible. The body and inertial forces are small compared to the viscous forces and can be neglected. With these assumptions in mind, next, the governing equations are given.

\subsubsection{Conservation of Mass (Continuity Equation)}

The physical principle of the conservation of mass can be stated as the total change of mass in a fixed arbitrary volume is equal to the net flux of mass across the boundary surfaces of the fixed volume [76]. This principle can be written mathematically as:

$$
\frac{\partial \rho}{\partial t}+\nabla \cdot(\rho \boldsymbol{u})=0
$$

where $\rho$ is the density, $\boldsymbol{u}$ is the velocity vector and $\nabla$ is the gradient operator. With the assumption of steady and incompressible flow (or negligible density change), Equation 3.1 simplifies to:

$$
\nabla \cdot \boldsymbol{u}=0
$$

This form of the continuity equation is often referred as the incompressibility constraint. 


\subsubsection{Conservation of Momentum}

The conservation of momentum principle states that the rate of increase in the momentum of the particles within an arbitrary fixed volume is equal to the summation of (i) rate of momentum change across the boundary surface by the bulk flow, (ii) surface forces exerted by the particles that are surrounding the fixed volume, and (iii) force on the particles within the fixed volume due to the gravity (body force) [76]. In mathematical form, the momentum conservation principle can be written as:

$$
\frac{\partial(\rho \boldsymbol{u})}{\partial t}=-[\nabla \cdot \rho \boldsymbol{u u}]+\nabla \cdot \sigma+\rho \boldsymbol{f}
$$

where $\sigma$ is the Cauchy stress tensor and $f$ is the body force vector. The Cauchy stress tensor can be broken up into hydrostatic and viscous parts.

$$
\sigma=-p I+\tau
$$

where $p$ is the hydrostatic pressure, $I$ is the unit tensor and $\tau$ is the viscous stress tensor. For a purely viscous, isotropic and incompressible fluid, Equation 3.4 becomes:

$$
\sigma=-p I+2 \eta \varepsilon
$$

where $\eta$ is the viscosity and $\varepsilon$ is the rate of strain (deformation) tensor defined as:

$$
\varepsilon=\frac{1}{2}\left[(\nabla \boldsymbol{u})+(\nabla \boldsymbol{u})^{T}\right]
$$

Second term on the right hand side of Equation 3.5 describes the stress-strain relationship (constitutive equation) of the material that is under consideration. This constitutive equation for an incompressible generalized Newtonian fluid can be written as 
$\tau=2 \eta \varepsilon$. The shear rate $(\dot{\gamma})$, which is a scalar term, is then given as $\dot{\gamma}=\sqrt{2(\varepsilon: \varepsilon)}$. For generalized Newtonian fluids, the viscosity, $\eta$, depends not only on temperature, but also on the shear rate. The shear rate and temperature dependency of the viscosity is discussed in Section 3.3.

Due to the higher viscosity and relatively lower density of polymers, the inertial forces and body forces in Equation 3.3 (the first and third term on the right-hand side) are small and can be neglected compared to the viscous forces. Thus, for a steady-state flow, Equation 3.3 is reduced to:

$$
\nabla \cdot \sigma=0
$$

For an isotropic, incompressible fluid with generalized Newtonian constitutive equation, the momentum conservation equation is further simplified to:

$$
-\nabla p+\nabla \cdot \eta\left[(\nabla \boldsymbol{u})+(\nabla \boldsymbol{u})^{T}\right]=0
$$

\subsubsection{Conservation of Energy}

The law of conservation of energy states that the rate of change of the energy in a system is equal to the summation of the rate of work done by the forces exerted on the system and the rate of heat content change in the system [76]. For an incompressible fluid, this statement can be written in mathematical form as:

$$
\rho C_{p}\left(\frac{\partial T}{\partial t}+\boldsymbol{u} \cdot \nabla T\right)=-\nabla \cdot \boldsymbol{q}+Q+\Phi
$$

where $T$ is the temperature, $C_{p}$ is the heat capacity (specific heat), $\boldsymbol{q}$ is the heat flux vector given as $\boldsymbol{q}=-(k I) . \nabla T$ where $\mathrm{k}$ is the thermal conductivity and $I$ is the identity 
matrix, $Q$ is the internal heat source, and $\Phi$ is the viscous dissipation. With steady-state flow and no internal heat source conditions, for an isotropic fluid, Equation 3.9 reduces to:

$$
\rho C_{p} \boldsymbol{u} \cdot \nabla T=\nabla \cdot(k \nabla T)+\Phi
$$

where heat generated due to viscous dissipation is given by:

$$
\Phi=(2 \eta \varepsilon): \varepsilon
$$

\subsection{Finite Element Formulation}

For the finite element simulation of the steady, incompressible flow of generalized Newtonian fluid, standard Galerkin formulation [58] was applied to the mass (Equation 3.2) and momentum (Equation 3.8) equations whereas an upwind scheme [59-65] was employed for the energy equation (Equation 3.10) to accurately capture the convection term. These equations (mass, momentum and energy) can be solved in a coupled manner (solve all equations simultaneously) or in a decoupled manner. In this work, decoupled approach was used, that is, for a given temperature vector, mass and momentum equations are solved to determine the velocity and pressure. Then, with the velocity vector thus determined, the energy equation is solved for temperature. This calculated temperature distribution is then employed to solve the momentum and mass equations and this iterative process continues till the convergence. 


\subsubsection{Finite Element Formulation of Mass and Momentum Equations}

In the finite element formulation of the mass and momentum equations, velocity and pressure within an element are approximated by the following equations:

$$
\begin{aligned}
u_{i}(\boldsymbol{x}) & =\boldsymbol{\psi}(\boldsymbol{x})^{T} \boldsymbol{u}_{i} \\
p(\boldsymbol{x}) & =\boldsymbol{\varphi}(\boldsymbol{x})^{T} \boldsymbol{p}
\end{aligned}
$$

where $\boldsymbol{\psi}$ and $\varphi$ are shape function vectors, and $\boldsymbol{u}_{\boldsymbol{i}}$ and $\boldsymbol{p}$ are the vectors including the nodal values of the velocity and pressure for an element, respectively. After applying the

standard Galerkin formulation [76], the weak (variational) form of Equations 3.2 and 3.8 in three dimensions can be written as:

Continuity equation:

$$
-Q_{1}^{T} \boldsymbol{u}_{\mathbf{1}}-Q_{2}^{T} \boldsymbol{u}_{\mathbf{2}}-Q_{3}^{T} \boldsymbol{u}_{\mathbf{3}}=0
$$

Momentum equation:

$$
\begin{aligned}
& \text { for } i, j=1,2,3 \\
& \qquad\left[K_{11}+K_{22}+K_{33}\right] \boldsymbol{u}_{\boldsymbol{i}}+K_{1 i} \boldsymbol{u}_{\mathbf{1}}+K_{2 i} \boldsymbol{u}_{\mathbf{2}}+K_{3 i} \boldsymbol{u}_{\mathbf{3}}-Q_{i} \boldsymbol{p}=\boldsymbol{F}_{\boldsymbol{i}}
\end{aligned}
$$

where the divergence matrix $\left(Q_{i}^{T}\right)$, the viscous diffusion matrix $\left(K_{i j}\right)$, pressure gradient matrix $\left(Q_{i}\right)$, and the force vector $\left(\boldsymbol{F}_{i}\right)$ are defined as:

$$
Q_{i}^{T}=\int_{\Omega} \boldsymbol{\varphi} \frac{\partial \boldsymbol{\psi}^{T}}{\partial x_{i}} d \Omega
$$




$$
\begin{gathered}
K_{i j}=\int_{\Omega} \eta \frac{\partial \boldsymbol{\psi}}{\partial x_{i}} \frac{\partial \boldsymbol{\psi}^{T}}{\partial x_{j}} d \Omega \\
Q_{i}=\int_{\Omega} \frac{\partial \boldsymbol{\psi}}{\partial x_{i}} \boldsymbol{\varphi}^{T} d \Omega \\
\boldsymbol{F}_{\boldsymbol{i}}=\oint_{\Gamma_{T}} \boldsymbol{\psi} F_{i}^{T} d s
\end{gathered}
$$

As discussed earlier, the body forces are neglected in this work. Thus, $\boldsymbol{F}_{\boldsymbol{i}}$ is a vector that only contains the boundary conditions. Since the viscosity in this work is a function of shear rate and temperature, the viscous diffusion matrix $(K)$ become a function of velocity which results in nonlinearity in Equation 3.15. Equations 3.14 and 3.15 may be written in the matrix form as:

$$
\left[\begin{array}{cccc}
\widehat{K}_{11} & K_{21} & K_{31} & -Q_{1} \\
K_{12} & \widehat{K}_{22} & K_{32} & -Q_{2} \\
K_{13} & K_{23} & \widehat{K}_{33} & -Q_{3} \\
-Q_{1}^{T} & -Q_{2}^{T} & -Q_{3}^{T} & 0
\end{array}\right]\left\{\begin{array}{c}
\boldsymbol{u}_{\mathbf{1}} \\
\boldsymbol{u}_{\mathbf{2}} \\
\boldsymbol{u}_{3} \\
\boldsymbol{p}
\end{array}\right\}=\left\{\begin{array}{c}
\boldsymbol{F}_{\mathbf{1}} \\
\boldsymbol{F}_{\mathbf{2}} \\
\boldsymbol{F}_{\mathbf{3}} \\
0
\end{array}\right\} \Longrightarrow \bar{K} \mho=\overline{\boldsymbol{F}}
$$

where

$$
\begin{aligned}
\widehat{K}_{11} & =2 K_{11}+K_{22}+K_{33} \\
\widehat{K}_{22} & =K_{11}+2 K_{22}+K_{33} \\
\widehat{K}_{33} & =K_{11}+K_{22}+2 K_{33}
\end{aligned}
$$

In order to solve the system of simultaneous linear equations obtained by the finite element method shown above, an iterative solver with preconditioned conjugate gradient method [77] was used in this work. 


\subsubsection{Finite Element Formulation of Energy Equation}

In the finite element formulation of energy equation, the temperature variable is approximated within each element in the form of:

$$
T(\boldsymbol{x})=\boldsymbol{\theta}(\boldsymbol{x})^{T} \boldsymbol{T}
$$

where $\boldsymbol{\theta}$ is the vector of shape function, and $\boldsymbol{T}$ is the vector including the nodal values of temperature of an element. After applying the standard Galerkin formulation [76], the weak (variational) form of the Equation 3.10 in three dimensions can be written as:

$$
H \boldsymbol{T}=\boldsymbol{F}
$$

where $H$, and $\boldsymbol{F}$ are defined as:

$$
\begin{gathered}
H=\int_{\Omega}\left[\sum_{j=1}^{3} \rho C_{p} \boldsymbol{\theta}\left(\boldsymbol{\psi}^{T} \boldsymbol{u}_{j}\right) \frac{\partial \boldsymbol{\theta}^{T}}{\partial x_{j}}+\sum_{j=1}^{3} k \frac{\partial \boldsymbol{\theta}}{\partial x_{j}} \frac{\partial \boldsymbol{\theta}^{T}}{\partial x_{j}}\right] d \Omega \\
\boldsymbol{F}=\int_{\Omega} \boldsymbol{\theta} \Phi d \Omega+\int_{\Gamma_{q}} \boldsymbol{\theta} \bar{q} d s
\end{gathered}
$$

where $\Phi$ is already described in Equation 3.11 and $\bar{q}$ is a constant heat flux boundary condition. As discussed earlier, in order to capture the convection term accurately in energy equation, an upwind scheme (SUPG method) [59-65] was employed. The weak form of the energy equation from SUPG method has an additional term on $H$ in Equation 3.17 [78]. This additional term may be written as:

$$
H_{a d d}=\int_{\Omega}\left[\rho C_{p} \tau\left(\sum_{i=1}^{3}\left(\boldsymbol{\psi}^{T} \boldsymbol{u}_{i}\right) \frac{\partial \boldsymbol{\theta}}{\partial x_{i}}\right)\left(\sum_{j=1}^{3}\left(\boldsymbol{\psi}^{T} \boldsymbol{u}_{j}\right) \frac{\partial \boldsymbol{\theta}^{T}}{\partial x_{j}}\right)\right] d \Omega
$$


where $\tau$ is the intrinsic time constant which is a function of velocity, Peclet number and length of an element.

\subsection{Viscosity Model}

In the present work, in order to capture the shear-thinning behavior of polymeric viscosity $\left(\eta_{s}\right)$, the shear viscosity of the amorphous and semi-crystalline polymers is represented by using the Cross model [79] (Equation 3.18).

$$
\eta_{s}=\frac{\eta_{0}}{1+\left(\frac{\eta_{0} \dot{\gamma}}{\tau^{*}}\right)^{1-n}}
$$

where $\eta_{0}$ is the zero-shear viscosity of the polymer, $\dot{\gamma}$ is the shear rate, $n$, and $\tau^{*}$ are material parameters. The temperature-dependence of shear viscosity $\left(\eta_{s}\right)$ are incorporated in the zero-shear viscosity $\eta_{0}$ and a modified WLF equation is used for the zero-shear viscosity [80] (Equation 3.19).

$$
\eta_{0}= \begin{cases}D_{1} \exp \left[-\frac{A_{1}\left(T-T_{a}\right)}{A_{2}+\left(T-T_{a}\right)}\right] & T>T_{a} \\ \infty & T \leq T_{a}\end{cases}
$$

where $T$ is the temperature, $T_{a}, D_{1}, A_{1}$, and $A_{2}$ are material constants. For each polymer, the material constants except $T_{a}$, in Equations 3.18 and 3.19 are obtained from the curve fit to the experimental data from the cone and plate viscometer. For all resins used in this work, the values of the material constants obtained from the curve fit were given in Table 2.4. In the WLF equation (Equation 3.19), $T_{a}$ is typically assumed to be equal to the glass transition temperature $\left(T_{g}\right)$ for amorphous polymers and the melting temperature $\left(T_{m}\right)$ for the semi-crystalline polymers. Therefore, as the temperature of 
the liquid polymer decreases and approaches to $T_{g}$ for amorphous polymers or $T_{m}$ for semi-crystalline polymers, the zero-shear viscosity predicted by the WLF equation increases rapidly. As shown in Equation 3.19, the zero-shear viscosity is taken to be infinite below the melting point of semi-crystalline polymers and below the glass-transition temperature of the amorphous polymers. Since an infinite value for the zero-shear viscosity cannot be used for the finite element simulation of polymer melting, it is assumed that the zero-shear viscosity is constant for temperatures less than glass-transition temperature for amorphous polymers or melting temperature for semi-crystalline polymers.

Following the approach which is commonly used to simulate the two-phase flow of a solid and liquid polymer combination in injection molding [80], in this work solid polymer was modeled as a very high viscosity fluid. The viscosity of the polymer below the glass transition temperature for amorphous polymers or melting temperature for semi-crystalline polymers predicted by the WLF equation is a few order magnitude higher than the viscosity of the polymer in liquid state. With such a large difference in the viscosity of the polymer, the compacted solid polymer only experience solid body rotation or translation with negligibly small shear rate $(\dot{\gamma})$.

\subsection{Numerical Simulations}

For the five screw configurations discussed in Section 2.1, a three-dimensional finite element simulation of the two phase flow of molten polymer and compacted polymer solid was performed for a portion of the screw channel using a software developed at Plastic Flow [81]. This software implements the numerical methods that are described in the earlier parts of this section. For the simulations, linear tetrahedral finite elements with velocity, pressure and temperature specified at each node and velocity specified at the centroid (bubble node) [82] were used. To efficiently solve the sets of equations resulting 


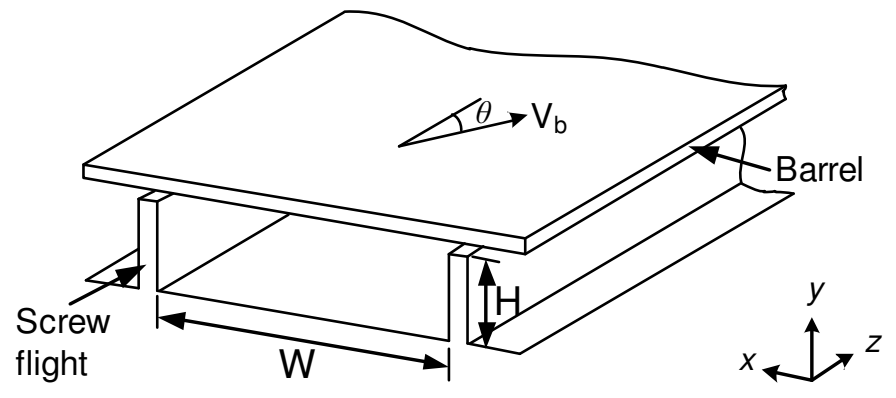

(a)

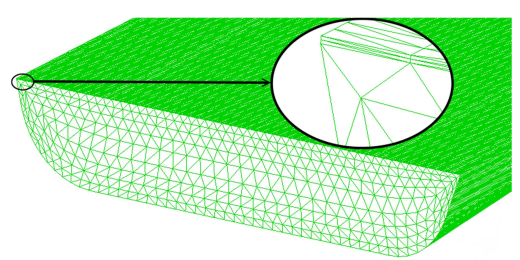

(b)

Figure 3.1: (a) Unwound helical channel of a single-screw extruder with the barrel as an infinite plate moving on top (b) Finite element mesh.

from the finite element method, the bubble nodes were eliminated at the element level by using the static condensation method [58].

In the finite element simulations, the coordinate frame was fixed on the screw and the helical screw channel was unwound into a straight channel to further simplify the simulations. The barrel was modeled as a plate moving on top of the straight channel at an angle equal to the helix angle of the screw (Fig. 3.1 (a)). Accordingly, to simulate the screw rotating at $60 \mathrm{rpm}$, the velocity of the top plate (barrel) was taken as $V_{x}=-0.060$ $\mathrm{m} / \mathrm{s}$ and $V_{z}=0.190 \mathrm{~m} / \mathrm{s}$ where $x$ is the cross-channel direction and $z$ is the downstream direction. Fig. 3.1 (b) shows a partial view of the finite element mesh used for the flow simulations. As shown in Fig. 3.1 (b), the finite element mesh had two thin layers of elements at the barrel surface with the thickness of each layer being half of the flight clearance. Effect of the thickness of these elements near the barrel surface on numerical predictions will be discussed later in Section 3.6.1. The flow domain for the simulations started at an axial distance of 6 diameters from the entrance of the extruder, which is the beginning of the compression section for all screws, and ends at axial distance of 14 diameters. Thus, for all screws except the 6-4-11CR2.8 screw, the flow domain simulated was the compression section of the extruder. For 6-4-11CR2.8 screw, the flow domain 
extended to the metering section of the extruder since this screw had a shorter compression section. For all simulations, unless stated, the temperature of the fully compacted solid polymer entering the channel was $30^{\circ} \mathrm{C}$, a uniform down-channel velocity was specified at the entrance of the unwound channel such that the flow rate measured in the screw freezing experiment was satisfied, and the zero-traction boundary condition was imposed at the exit.

\subsection{Use of Graphical Processing Unit for Finite} Element Computations

Even though the software used [81] can simulate the flow of molten polymer in an extrusion die in less than an hour on a personal computer (PC), when the software was used to simulate a two-phase flow of molten polymer and compacted solid in a single-screw extruder channel, convergence of the two-phase flow simulation was slow. For a two-phase simulation with a mesh of 1.03 million elements, it took about 484 hours on a Pentium IV $3-G H z$ PC.

Since the one of the goals of this work is to investigate the effect of various processing conditions and material properties on the melting of a polymer in a single-screw extruder, a large number of flow simulations were required for this investigation. With each flow simulation requiring more than 20 days on a Pentium IV 3-GHz PC, the completion of this research would not have been possible on a Pentium IV 3-GHz PC. Also, it is difficult to expect a PC to run a simulation continuously for 20 days. Many of the flow simulations we started got terminated in between because of technical difficulties. With these difficulties encountered in completing the simulations on a PC, more robust computational resources were sought to complete this investigation. 
Through a grant from National Center for Supercomputing Applications (NCSA), the two-phase flow simulations in the single-screw extruder were attempted on the Condor Pool cluster at Purdue University [83]. The completion of a two-phase flow simulation on the Condor Pool cluster, which consisted of 3-GHz CPUs, also required about 484 hours. We were able to successfully complete only one two-phase flow on the Condor Pool cluster. Other flow simulations we submitted on Condor Pool cluster got terminated after running for several days on the cluster. At this point of investigation, it was clear that for successful completion of this investigation the computation time required for flow simulations must be reduced by at least an order of magnitude.

The computation time for a simulation of melting and flow in single-screw extruders was attempted to be reduced first by developing a MPI (Message Passing Interface) code. MPI is a communication protocol to program parallel computers in a cluster. Solution of a large number of simultaneous equations obtained from the finite element method (FEM) is a highly parallel operation, thus suitable for MPI application. In order to develop and test the MPI code, a small cluster in the Department of Mathematical Sciences at Michigan Technological University consisting of 20 computers, each with $3.0 \mathrm{GHz}$ CPUs and $2 \mathrm{~GB}$ memory, were used. For testing purposes, an isothermal case simulation was used with the same finite element mesh and boundary conditions that was submitted to the Condor Pool cluster at Purdue University [83]. In the isothermal case simulations, the material properties, particularly the shear viscosity, are not a function of temperature, thus the computation time is in the order of minutes. For comparison, the isothermal case simulation was submitted to one, ten and twenty computers, respectively. The computation times to simulate the flow of a polymer in single-screw extruders were 320 , 100 and 90 minutes with a use of one, ten and twenty computers in the cluster, respectively. As given by the results, the computation time decreased with an increase in the number of computers used for the isothermal simulations. However, the computation time was 
decreasing at a smaller rate compared to the increase in the computation nodes. The main reason that caused this bottleneck was the communication time and the bandwidth between the computers in the cluster.

In order to reduce the computation time required for simulation of the two-phase flow in an extruder channel by a large amount, next, a Graphical Processing Unit (GPU) on the graphics card of a PC was used for solving the set of linear equations obtained by finite element discretization of the two-phase problem. Finite element formulation of the polymer melting in a single-screw extruder results in a system of simultaneous equations. Because of the highly sparse and banded nature of the matrix for the system of simultaneous linear equations obtained by finite element method (FEM) as discussed earlier, an iterative solver with preconditioned conjugate gradient method was used. Each iteration of the conjugate gradient method [77] requires multiplication of the system matrix of the simultaneous linear equations with the current estimate of the solution. Matrix multiplication with a vector is highly parallelizable operation because it requires dot product of each row of the matrix with the vector, which can be computed concurrently for several rows at a time. The number of simultaneous linear equations obtained in a finite element simulation of the melting in a single-screw extruder is generally more than a million. Solution of such a large number of simultaneous equations by conjugate gradient method is a highly memory intensive operation, and is therefore particularly suitable for GPU application.

In this work, a computer with Intel Core 2 Quad (2.33 GHz, 4MB cache), 8GB DDR2/800 dual mode RAM and NVIDIA GeForce GTX 260 graphics card in the Department of Mathematical Sciences at Michigan Technological University was used. The CUDA 2.0 (Compute Unified Device Architecture) architecture from NVIDIA was used to accelerate the matrix-vector multiplication and other matrix operations required in each iteration of a preconditioned conjugate gradient method for solving the set of 
linear equations. BLAS1 (Level 1 Basic Linear Algebra Subprograms) functions was called from the CUBLAS library. In order to perform the sparse-matrix vector multiplication operation, a CUDA code developed by Bell and Garland [84] was modified and embedded into the main program.

A performance study was performed to compare the computation power of Central Processing Unit (CPU) and GPU. In this performance study, global stiffness matrix obtained from the FEM, which is sparse in nature, was multiplied with a random vector. Specifications of the computer used in computations were given earlier in the previous paragraph. Global stiffness matrix from the FEM discretization has 771,088 rows and columns with total non-zero elements of $43,543,936$. Performance study showed that Intel Core 2 Quad (2.33 GHz, 4MB cache) performed $0.96 \mathrm{GFLOP} / \mathrm{s}$ (giga-floating point operations per second), whereas the NVIDIA GeForce GTX 260 graphics card performed 12.19 $\mathrm{GLOP} / \mathrm{s}$, an increase of 12.7 times. Operations like dot product and sparse matrix-vector multiplication have very low computational intensity, that is, the number of floating point operations per memory operation is very low. Thus, these operations entirely limited by the memory bandwidth. Because of that reason, it is also important to compare the CPU and GPU computations with some memory bandwidth benchmarks. Performance study showed that NVIDIA GeForce GTX 260 graphics card has a bandwidth of 128.1 Gigabytes/s, outperformed the bandwidth of 9.8 Gigabytes/s on an Intel Core 2 Quad CPU.

As shown by the performance study, with the use of the GeForce GTX 260 GPU from NVIDIA, the computation time for a simulation of melting and flow in single-screw extruders is expected to be less than one-tenth $(10 \%)$ of the computation time required for the same simulation using CPU of a personal computer. As expected, with the use of the GPU, only 16 hours of computation time was required for the finite element simulation of the two-phase flow in the extruder channel with about 1.03 million elements in the 
finite element mesh; an improvement from 484 hours with CPU to 16 hours with GPU. This improvement on the computation was much higher than the improvement on one sparse-matrix vector multiplication discussed earlier because the solution to the system of equations also included dot product and reduction operations of vectors and also the computation time on the cluster at the Purdue University [83] included the time for check pointing which is needed for a stable continuation of simulations with long computation times. With the reduction in the computation time required for the two-phase flow simulation by a factor of 30 ( 20 days using CPU to 16 hours using GPU), an investigation of the effect of processing conditions and material properties on polymer melting as well as the optimization of a screw geometry in single-screw extruders became feasible.

\subsection{Numerical Simulation Results}

In this section, a comparison of the experimental data for the ABS and LDPE resins with the numerical predictions is presented. A numerical investigation of the effect of various processing conditions and material properties on melting of polymers in a single-screw extruder is also given in this section. Material properties of the resins given in Section 2.3.1, and the processing conditions during the experiments discussed in Section 2.1.1 were used to simulate the flow of polymers in a single-screw extruder. As mentioned earlier, the simulations were performed for axial screw channel lengths of eight diameters. The flow domain started at an axial distance of 6 diameters from the entrance of the extruder. Accordingly, in the simulation results shown in this section, the cross-sections are labeled from 6 to 14 . It should be noted here that various parameters describing the geometry of the screws used in this study was already given in Section 2.1. 


\subsubsection{ABS Resin Results: 6-8-7CR2.8 Screw}

As discussed in Section 2.1, five different screw configurations were used in the Maddock screw-freezing experiments for the ABS resin. The numerical simulations for the 6-8-7CR2.8 screw with the boundary conditions discussed in Section 3.4 were performed first. That is, the boundary conditions were - constant barrel and screw temperature of $230{ }^{\circ} \mathrm{C}$, and flow rate of $59.7 \mathrm{~kg} / \mathrm{h}$. The experimental melting profile obtained from the Maddock screw-freezing experiments for the 6-8-7CR2.8 is already shown in Fig. 2.4. For the $A B S$ resin, the sensitivity of the polymer melting in a single-screw extruder to material properties and processing conditions was analyzed around this basic case by changing one of the material parameters or processing conditions, while keeping all other parameters the same as those for the basic case.

Fig. 3.2 shows the predicted temperature distribution for the basic flow conditions along with the experimental data from Fig. 2.4. It should be noted that the scale bar of the temperature in Fig. 3.2, and also in other plots of the predicted temperature in this section, is not linear. The glass transition temperature of the ABS resin is $100{ }^{\circ} \mathrm{C}$ and as discussed in Section 2.3.1, almost no flow occurs below $150{ }^{\circ} \mathrm{C}$. Therefore, the region below $150{ }^{\circ} \mathrm{C}$, shown with blue color in Fig. 3.2 and other temperature plots, is solid polymer, whereas other colors show the temperature of polymer melt. For ease of comparison, for all temperature plots in this section, the temperature scale is divided into the same intervals, and all intervals are assigned with the same color. It should be noted that the cross-sectional plane for the photographs in Figs. $2.2-2.7$ and that for the simulations results presented in this paper were slightly different. For the photographs shown in Figs. 2.2-2.7, the solidified polymer from the screw was sliced along the extruder axis, whereas, the cross-sections in simulation results were in a plane perpendicular to the axis of the straightened screw channel. 

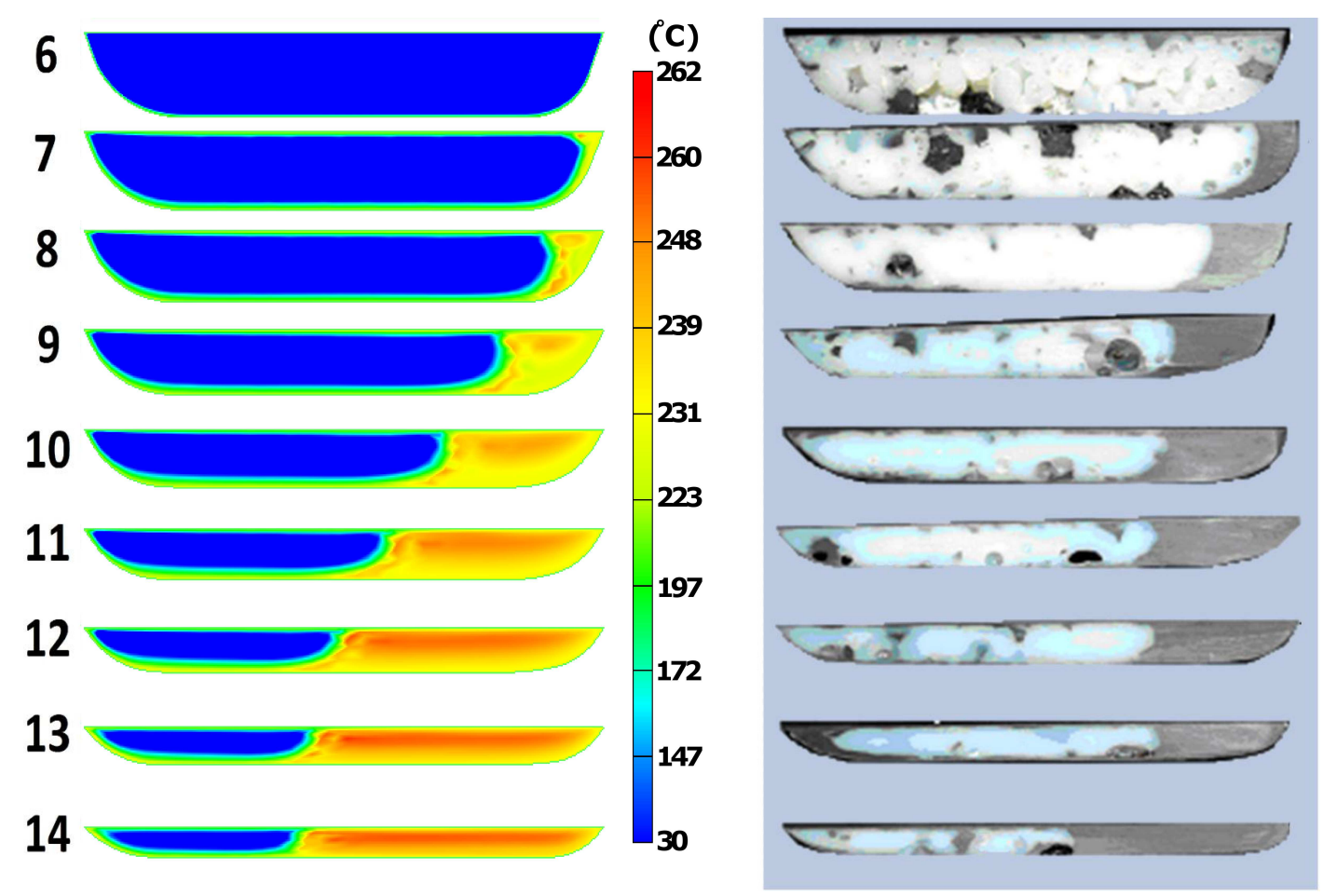

Figure 3.2: Predicted temperature distribution in the melting section of the extruder for a constant screw temperature of $230{ }^{\circ} \mathrm{C}$ (left) and the corresponding experimental data from Fig. 2.4 (right).

The melting pattern in the photographs in Fig. 2.4 as well as that in the simulation results in Fig. 3.2 follow the Maddock mechanism. In the experiments (Fig. 2.4) as well as in the simulation (Fig. 3.2) molten polymer between the hot barrel and the compacted solid pellets was observed to accumulate near the active flight and the width of the melt pool gradually increased in the down-channel direction whereas that of the solid bed decreased. Numerical simulations (Fig. 3.2) and experimental results (Fig. 2.4) also show the tendency of the melt pool to penetrate under the solid bed near the screw surface. It is noted that the temperature of the molten polymer in this region underneath the solid bed is quite high, indicating the high shear rate (and hence, higher viscous dissipation) in this region of the recirculating flow of the melt pool. 


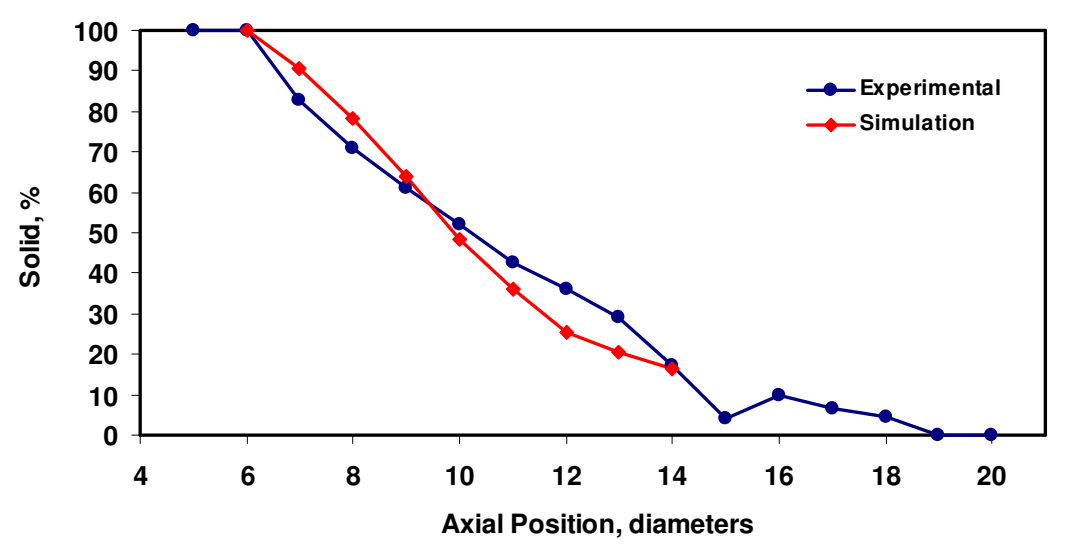

Figure 3.3: Comparison of experimental and numerical melting profile.

Fig. 3.3 shows the variation of the solid fraction along the screw channel. For the simulation results in Fig. 3.2 as well as the experimental data in Fig. 2.4, the solid fraction presented in Fig. 3.3 was measured as described in Section 2.1.1. The calculation of the solid fraction described in Section 2.1.1 was also formulated in Equation 2.4. It should be noted here that in Fig. 3.3, at an axial distance of 15 diameters, the solid percentage appears to be lower than that at 16 diameters. This is due to the solid bed break-up as mentioned earlier in Section 2.1.1.

Even though, the graph in Fig. 3.3 clearly shows that the predicted melting profile in Fig. 3.2 is in good agreement with the corresponding experimental data in Fig. 2.4, the simulation results have some differences compared to the experimental data. In particular, the solid bed width in numerical simulation decreased at a different rate than that in the experimental data in Fig. 2.4. That is, the predicted melting rate in the numerical simulation is different than the melting rate in the experiments. This difference in melting rate is also evident in Fig. 3.3, which shows the variation of solid fraction along the screw channel. One of the possible reasons for this difference in the melting rate is that the heat capacity for the ABS resin was assumed to be constant in the simulations whereas in reality it rapidly increases near the glass transition temperature of the amorphous polymers like $A B S$ as the temperature increases. 
The predicted cross-channel velocity distribution corresponding to the melting profile shown in Fig. 3.2 is given in Fig. 3.4. In Fig. 3.4, the length of all the vectors is the same, and the color of the arrow shows the magnitude of the cross-channel velocity ( $x$ and $y$ components). Following Maddock's melting mechanism [10], Fig. 3.4 shows that the polymer in the thin layer of molten polymer between the solid compact and the barrel surface is dragged towards the active flight of the screw to form a melt pool near this flight. As the polymer from the melt film enters the melt pool, the increase in pressure in the melt pool forces the solid bed to move towards the passive flight of the screw channel. The cross-channel velocity distribution in Fig. 3.4 also shows the recirculatory nature of the flow in the melt pool. The molten polymer in the melt pool moves toward the active flight with a relatively higher velocity in the upper portion of the channel and it moves toward the passive flight in the lower portion of the channel with a relatively smaller velocity.

Predicted pressure profile corresponding to the melting profile shown in Fig. 3.2 is given in Fig. 3.5. It should be noted here that a separate scale bar was used below the each of the cross-sections in Fig. 3.5 to clearly show the predicted pressure distribution. It is evident from Fig. 3.5 that the pressure in the melt pool gradually decreases from active flight to passive flight. In the solid bed, the pressure is lower than that in the melt pool, and remains relatively unchanged throughout the solid bed in the complete melting section of the screw channel.

Fig. 3.6 (a) shows the streamline for three particles starting at different locations at the channel entrance. At the channel entrance the polymer temperature is specified to be $30{ }^{\circ} \mathrm{C}$. That is, the channel cross-section at the entrance is completely occupied by the compacted solid polymer. Starting from the three different locations in the solid-bed, all three particles initially move towards the barrel surface and the passive flight. As these particles in the solid-bed continue to move towards the passive flight and towards the 


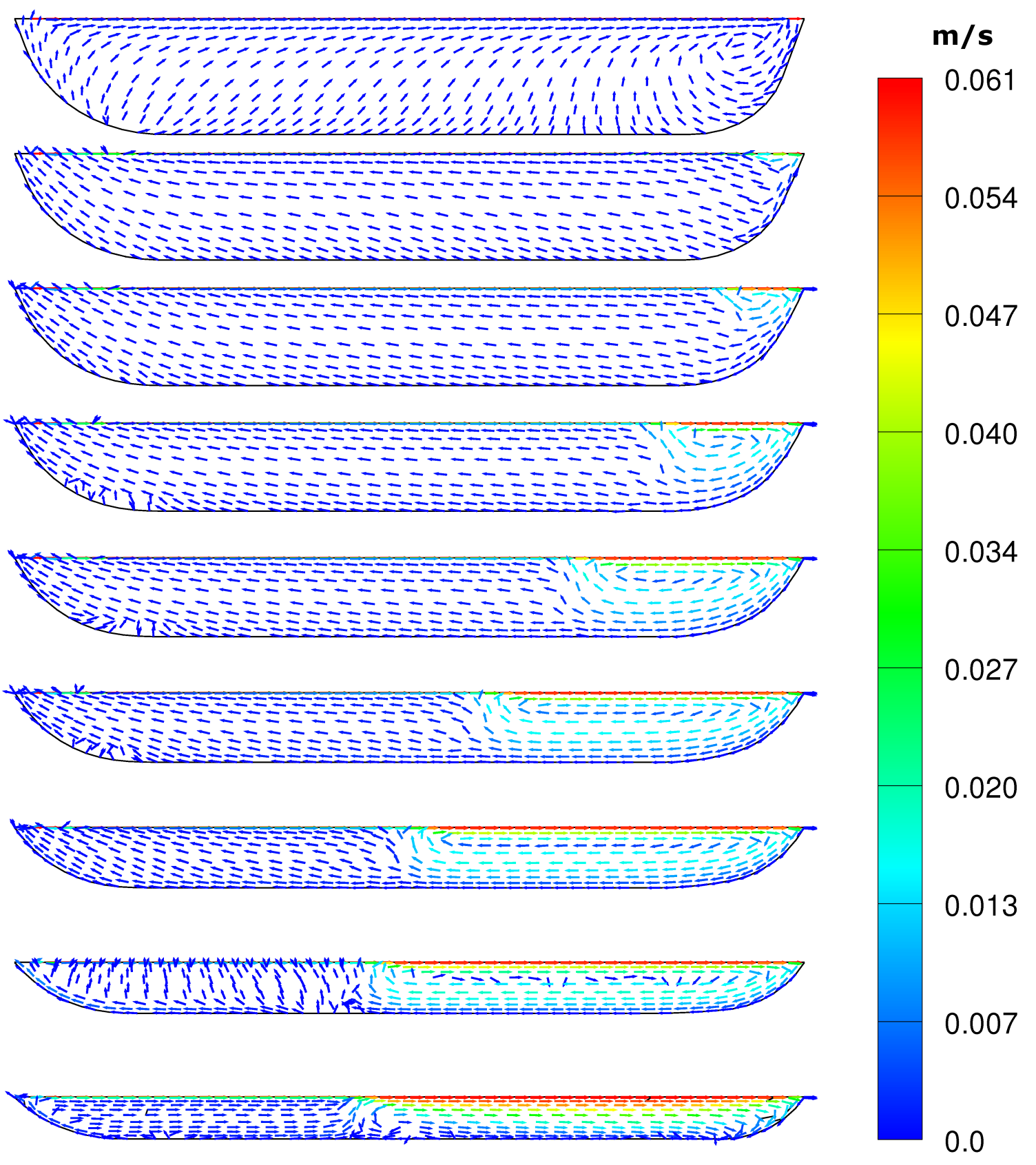

Figure 3.4: The predicted velocity profile corresponding to the melting profile shown in Fig. 3.2. 


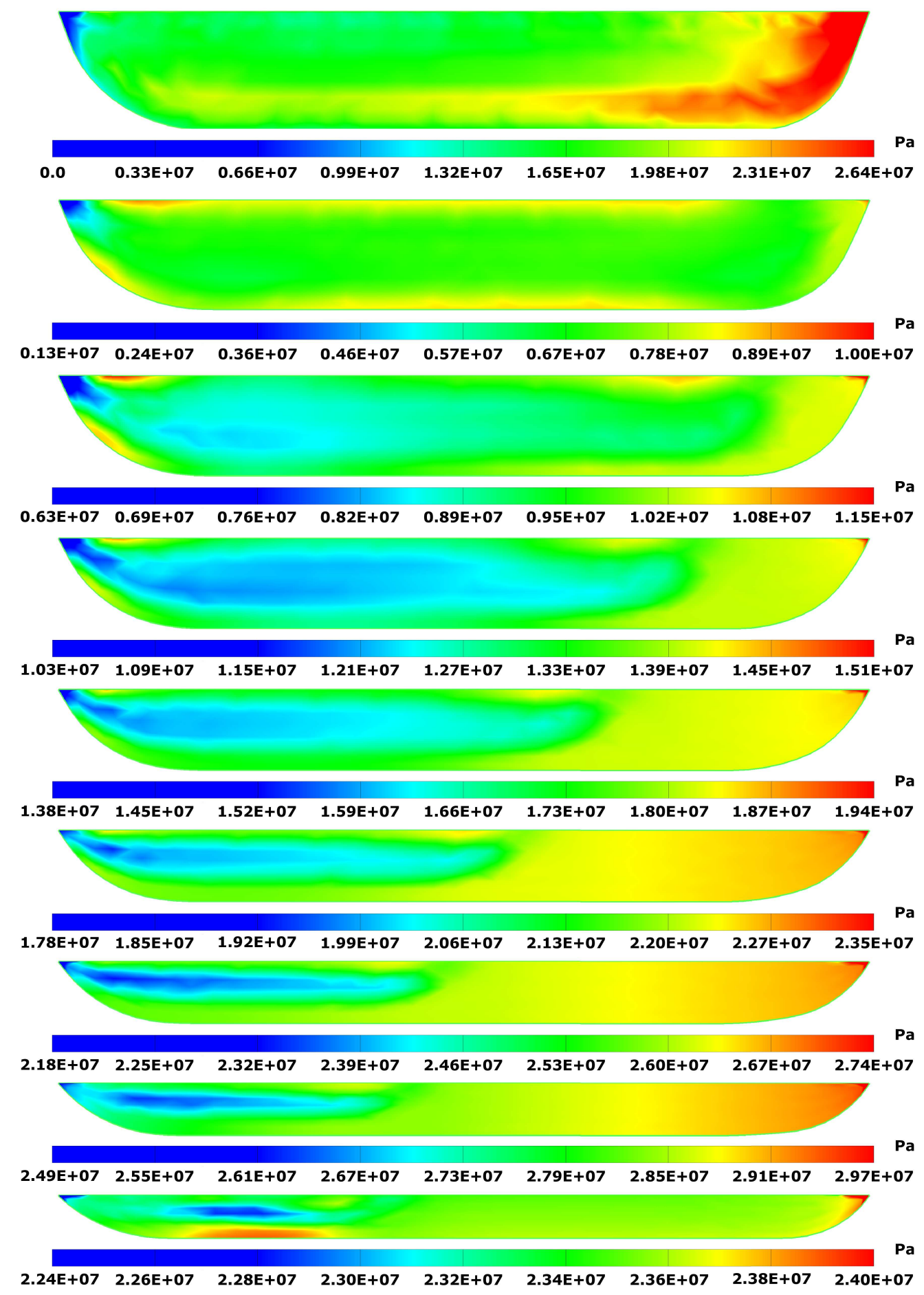

Figure 3.5: Predicted pressure profile corresponding to the melting profile shown in Fig. 3.2. 


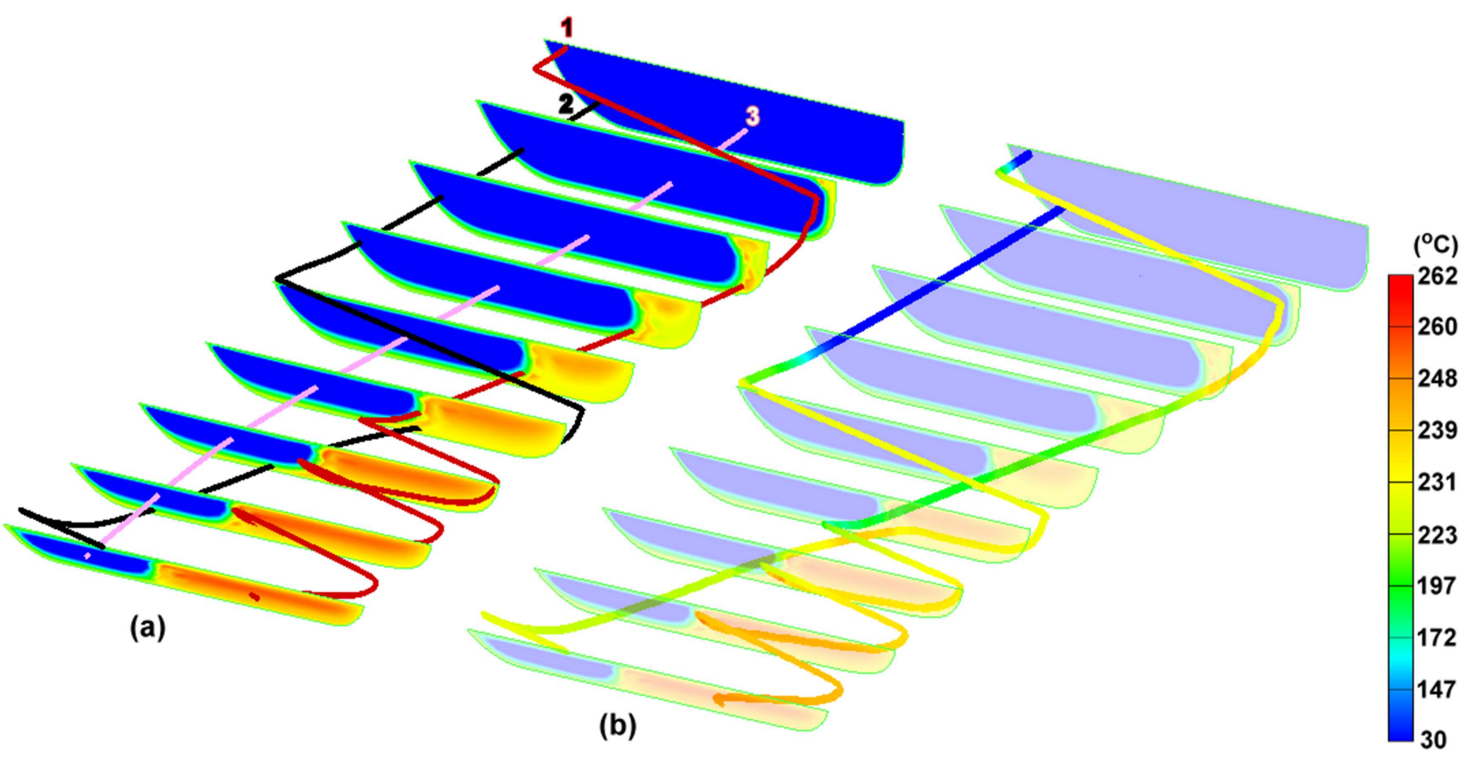

Figure 3.6: Predicted (a) polymer particle paths and (b) temperature of particle \#1 and \#2 in the melting section of the extruder.

barrel surface, they eventually reach the thin melt film near the barrel surface. Once the solid polymer particles reach the thin melt film, the temperature of the particles increases above the softening point. The polymer particles are then dragged along the barrel surface as part of the thin film, finally reaching the melt pool near the active flight. In Fig. 3.6 (a), particle \#1, which enters the channel near the passive flight and close to the barrel surface, reaches the melt film just beyond the entrance. Particle \#2, which enters the channel away from the barrel surface, continues to move towards the passive flight and barrel surface and reaches the melt film near the fifth cross-section in Fig. 3.6 (a). However, particle \#3, which starts near the middle of the cross-section at the entrance, has not reached the melt film even till the end of the compression section.

Two types of flow patterns were observed for the polymer particles in the melt pool. Most particles in the melt pool (such as particle \#1) continue to circulate in the melt pool as they move downstream. But, some of the particles near the screw surface (such as particle \#2), which reach the region of the melt pool where the interface between the 
solid bed and melt pool meets with the screw surface, become part of the thin melt film on the screw surface underneath the solid-bed. However, it was noticed that some of the particles in this small region where the melt pool penetrates below the solid-bed, do re-enter the melt pool. Only the particles in this region which are very close to the screw surface go underneath the solid bed. These particles then become part of melt film on the barrel surface; and then re-enter the melt pool for the second time. These two types of flow patterns, namely, recirculation with in the melt pool, and recirculation around the solid-bed, were experimentally observed by Bruker and Balch [43].

Fig. 3.6 (b) shows the variation in the temperature of particles \#1 and \#2 as they flow along the screw channel. As expected, the temperature of the two particles remains relatively unchanged till they approach near the melt film. For particle \#1 as well as \#2, as they approach the melt film, the temperature increases rapidly beyond the softening point of the polymer. As the two particles travel downstream, their temperature continues to increase while flowing in the melt film and also during recirculation around the melt pool. After circulating around the melt pool, as the two particles reach the solid-bed near the bottom of the screw channel, the particles temperature then decreases. For particle $\# 1$, as it moves upward towards the barrel surface, the temperature increases again. While particle \#2 goes underneath and around the solid-bed, its temperature remains low, and starts to increase only after it approaches the melt film near the barrel surface.

In the next section, the effect of the finite element mesh on the numerical results is investigated. Later, the sensitivity of melting profile to screw and barrel temperatures as well as to different thermal conductivity, heat capacity, shear viscosity, and flow rate values is examined. 


\section{The Effect of Finite Element Mesh on Numerical Predictions}

The finite element mesh used for all simulations discussed in this section had two thin layers of finite elements at the barrel surface with the thickness of each layer being equal to the half of the flight clearance as shown in Fig. 3.1 (b). In order to investigate the effect of these thin layers of elements at the barrel surface, another simulation was performed without these two layers of thin elements in the finite element mesh. The temperature distribution from this simulation with the flow conditions same as those for the basic flow conditions (Fig. 3.2) is shown in Fig. 3.7. It can be observed from Fig. 3.7 that the melting rate is significantly higher if the finite element mesh does not include the two thin layers of elements at the barrel surface. Since the velocity boundary condition is enforced at the barrel surface, the thickness of the elements that are adjacent to the barrel surface is important because it largely affects the shear rate and the shear stress in the thin film of polymer between the solid bed and barrel surface. When a mesh without a layer of thin finite elements at the barrel surface was used for the simulation, since the size of the elements adjacent to the barrel surface was bigger, the shear rate on the barrel surface was lower which resulted in a less viscous dissipation near the barrel surface. Lower viscous dissipation on these elements resulted in lower temperatures which causes higher shear viscosity. Thus, even though the heat generated through the viscous dissipation decreases, the shear stress near the barrel surface increases which resulted in a higher acceleration of the solid bed in the down-channel direction. This higher acceleration of the solid bed for the mesh without thin layers at the barrel surface is also evident in Fig. 3.8, which shows the average solid bed velocity for the simulation with the two different meshes. This downstream acceleration of the solid bed resulted in elongational deformation in the solid bed. Since the solid polymer is modeled as a high viscosity fluid in the present work, this elongational deformation of the solid bed generates a large amount of heat within the solid bed. This large amount of heat generated erroneously in the simulation without 


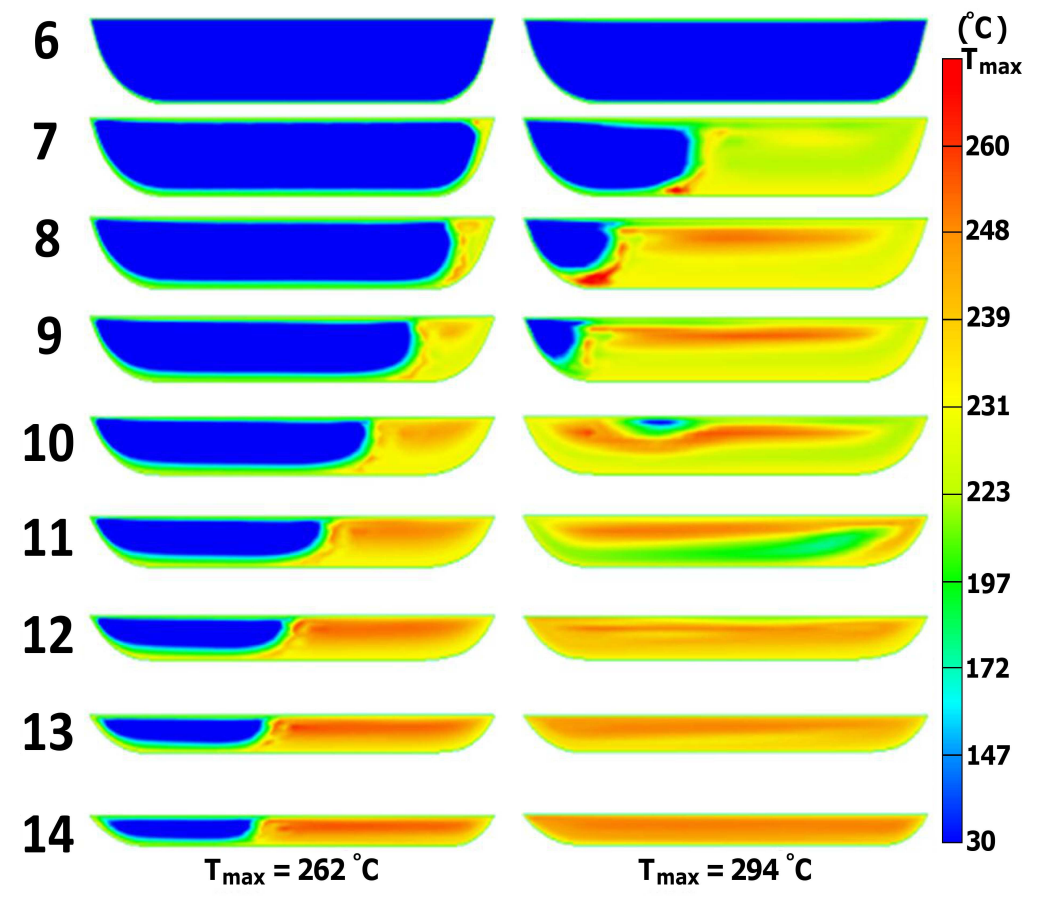

(a)

(b)

Figure 3.7: Predicted temperature distribution in the melting section of the extruder for the finite element mesh (a) with two thin layers of elements (Fig. 3.2), and (b) with no thin layer of elements, at the barrel surface.

thin layer of elements on the barrel surface melted the solid bed in a short length of the extruder channel in Fig. 3.7 (b).

In order to investigate the effect of the number of layers near the barrel surface, another finite element mesh with one thin layer of elements at the barrel surface with the thickness of this layer being equal to the flight clearance was also used to simulate flow with the basic conditions. The predicted temperature distribution obtained from this simulation was similar to that in Fig. 3.2. With one layer of thin finite elements on the barrel surface, the predicted pressure and velocity distributions were also similar to those obtained using the mesh with two layers of thin elements on the barrel surface. Even though the predictions with one layer and with two layers of elements on barrel 


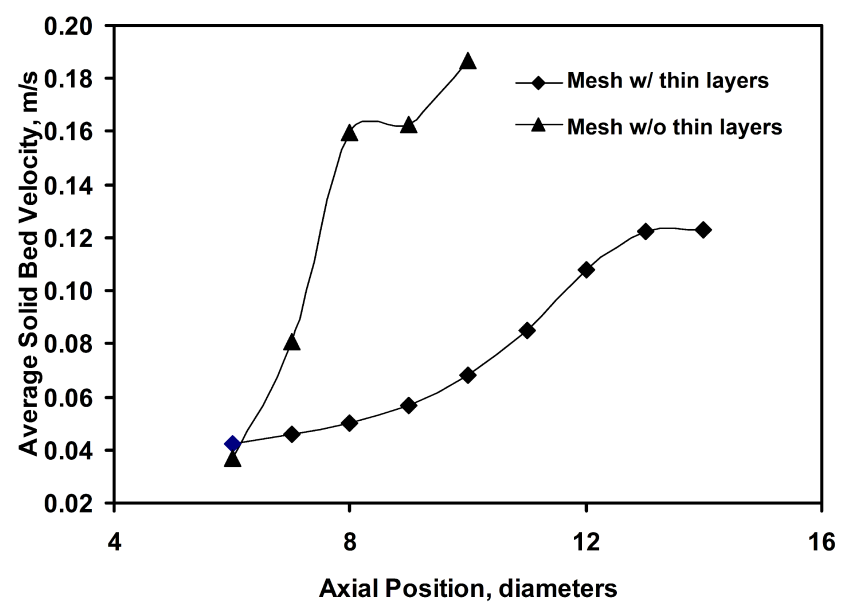

Figure 3.8: Predicted average down-channel velocity of the solid-bed in the melting section of the extruder for different finite element mesh.

surface were qualitatively similar, the simulations with two layers of elements were in better agreement with experimental data. Due to the limitation on GPU memory and the larger computation time required, three or more thin layers of elements on the barrel surface could not be used.

\section{The Effect of Screw Temperature on Melting Profile}

In most practical situations, the screw temperature in a single-screw extruder is not controlled and is determined by various parameters such as material properties, screw geometry and processing conditions [85]. Furthermore, since the screws in most commercial extruders are not equipped with thermocouples, finding an accurate estimate of screw temperature in an extruder is difficult. In various analysis of the polymer temperature in extruder channels in the literature, the screw is either assumed to be adiabatic $[86,87]$ or to have a constant temperature [87]. However, in extruders the screw temperature near the exit is almost the same as the polymer melt temperature, whereas near the hopper the screw temperature is close to the cooling jacket temperature [86]. Some of the experimental investigations of screw temperature in single-screw extruders 
in the literature showed that near the beginning of the compression (melting) section the screw temperature is lower than that at discharge and towards the end of the metering section, the screw temperature slightly exceeds the set metering section barrel temperature $[51,88]$. However, the change in screw temperature along the extruder channel can be quite complicated. For example, in Derezinski's work [86], the screw temperature did not increase consistently along the extruder channel and a decrease in the screw temperature near the beginning of the compression section was reported. Thus, more research needs to be done to experimentally measure the screw temperature at various locations along an extruder channel. Due to this inability to accurately establish the axial temperature distribution along the screw, in the simulations presented in this section only a constant screw temperature boundary condition was employed.

Figs. $3.9(\mathrm{a})-(\mathrm{c})$ show the temperature distributions at various cross-sections of the screw channel for screw temperatures of 230,250 , and $270{ }^{\circ} \mathrm{C}$. The temperature distribution for the screw temperature of $230{ }^{\circ} \mathrm{C}$ was also shown in Fig. 3.2. Since the discharge temperature from the extruder was measured at $264{ }^{\circ} \mathrm{C}$ in the experiment, the screw temperature is not expected to exceed far beyond $270{ }^{\circ} \mathrm{C}$ in the experiment. As shown in Fig. 3.9, an increase in screw temperature results in only a minor change in melting profile and the percentage of the solid polymer at the same cross-section slightly decreases. This slight decrease in solid fraction with an increase in screw temperature is also evident in Fig. 3.10, which shows the variation of solid fraction along the extruder channel for the three screw temperatures. It is also evident from Fig. 3.9 that an increase in screw temperature increases the melt pool temperature significantly.

To further investigate the effect of screw temperature on melting and flow of polymers, predicted pressure and down-channel velocity distribution at various locations along the screw channel is analyzed for the screw temperatures of 230,250 , and $270{ }^{\circ} \mathrm{C}$. The predicted down-channel velocity results for these screw temperatures are shown in Fig. 


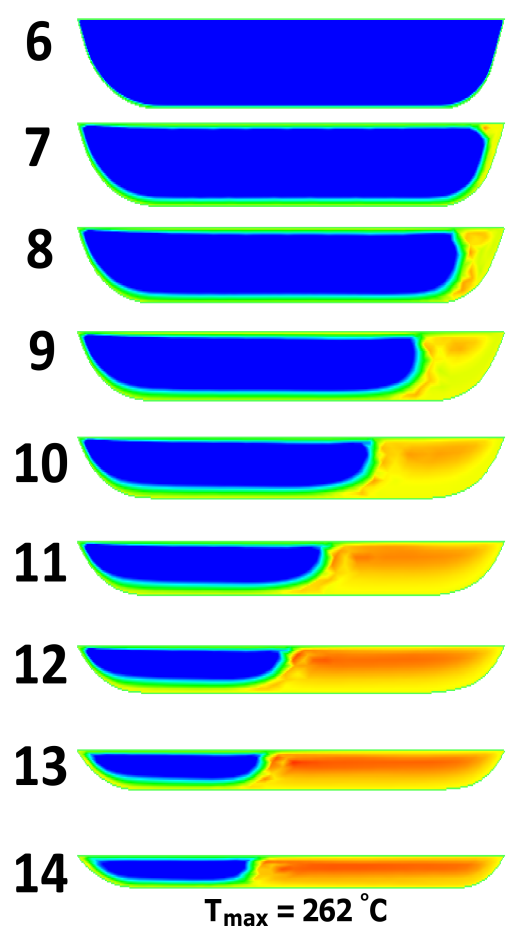

(a)
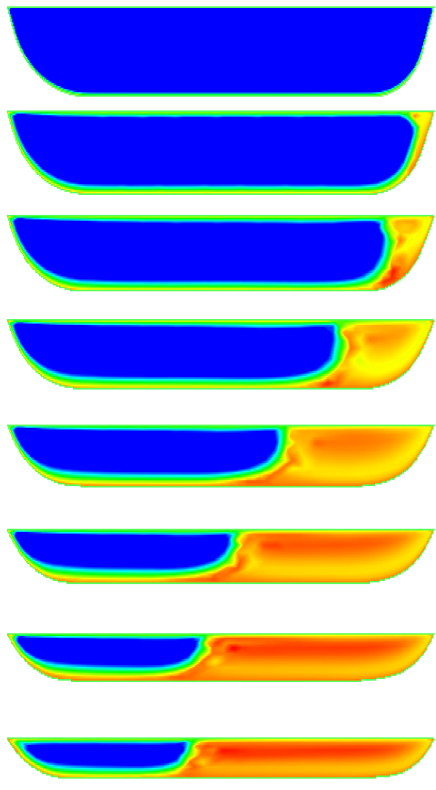

$\mathrm{T}_{\max }=276^{\circ} \mathrm{C}$

(b)

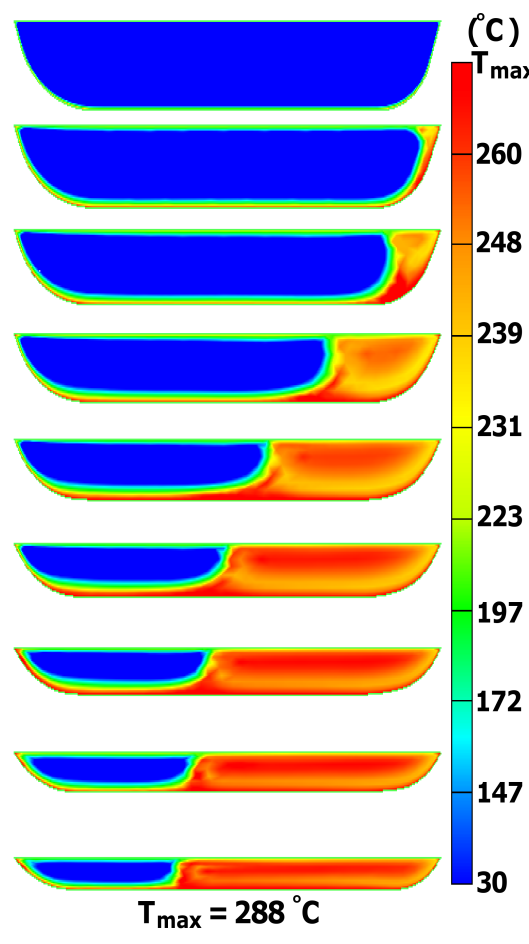

(c)

Figure 3.9: Predicted temperature distribution in the melting section of the extruder for constant screw temperatures of (a) 230 , (b) 250 , and (c) $270{ }^{\circ} \mathrm{C}$.

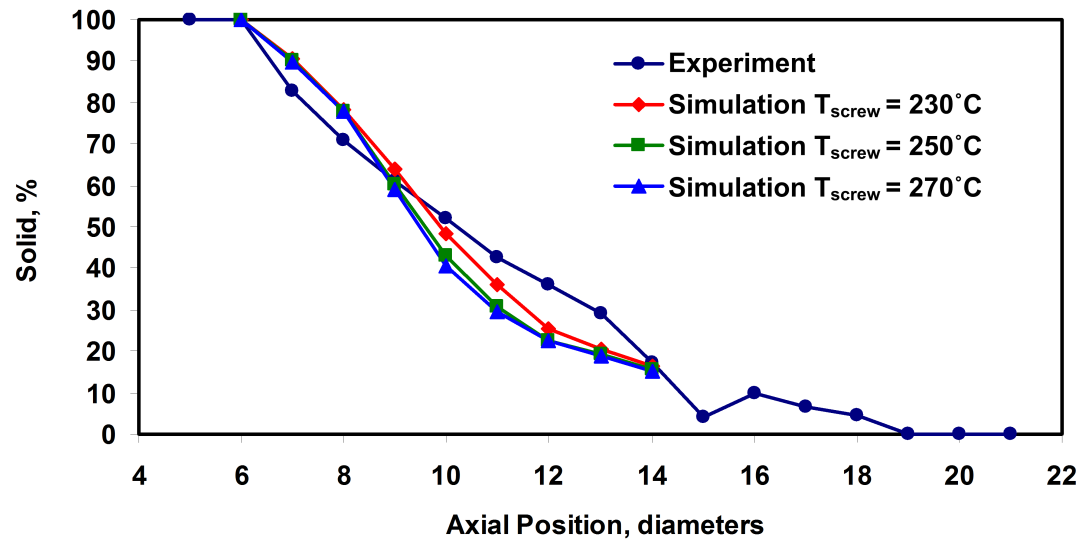

Figure 3.10: Comparison of predicted melting profiles for screw temperatures of 230, 250, and $270{ }^{\circ} \mathrm{C}$. 
3.11. The velocity scale bar in Fig. 3.11 is divided into the same intervals and all intervals are assigned with the same color. The dark blue color in the velocity scale bar refers to a flow in the upstream direction (back-flow). As shown in Fig. 3.11, the solid-bed velocity increases as the solid bed moves along the screw channel. That is, the solid bed moves faster in the later part of the melting section. This increase in the down-channel velocity of the solid bed is also shown by Fig. 3.12. The acceleration of the solid bed was experimentally observed by Bruker and Balch [43] and Zhu and Chen [89]. Bruker and Balch [43] observed the acceleration of the solid bed in the melting section by a flow visualization method. Zhu and Chen [89] implemented a tracer particle technique and observed a slow and steady acceleration of solid bed in the early part of the melting region whereas a sharp acceleration was observed in the later part of the melting section. These experimental observations in the literature were in line with the predictions in the simulation results shown in Figs. 3.11 and 3.12. Furthermore, it is evident from Fig. 3.11 that the amount of back-flow near the screw surface in the melt pool increases with increasing screw temperature. As illustrated in Fig. 3.11, at a screw temperature of 230 ${ }^{\circ} \mathrm{C}$, the predicted down-channel velocity distribution indicates no back-flow whereas an increase in the screw temperature resulted in a back-flow region near the screw surface in the melt pool as indicated by the dark blue color. Back-flow in the melting section of a single-screw extruder has been observed experimentally [43] as well as in numerical simulations [90].

Figure 3.13 shows the effect of the screw temperature on the predicted pressure at various locations of the screw channel along with the corresponding experimental data. It should be noted here that in experiments, pressure transducers were tap mounted on the inside surface of the extruder barrel. As the screw rotates inside the barrel, these pressure transducers measure a time dependent pressure profile at an axial cross-section of the screw channel. In simulations, the average pressure values across the same axial cross-section of 


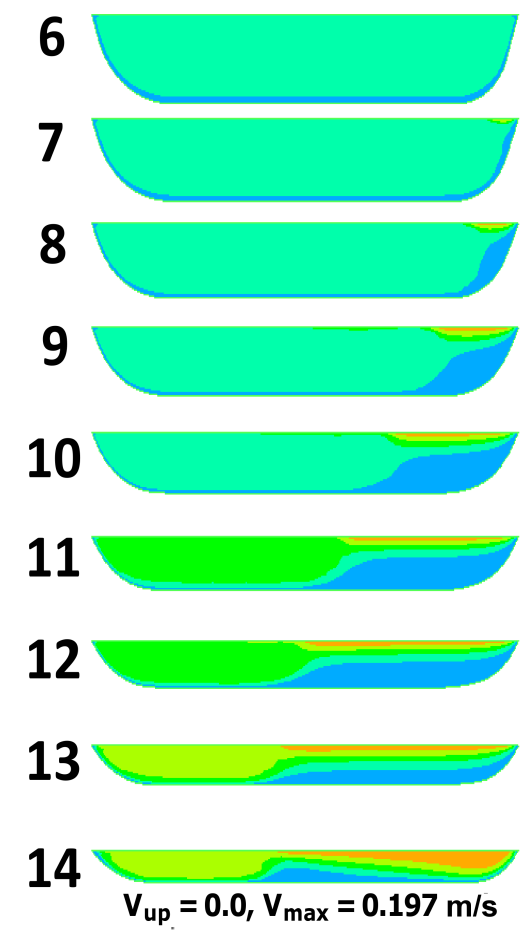

(a)

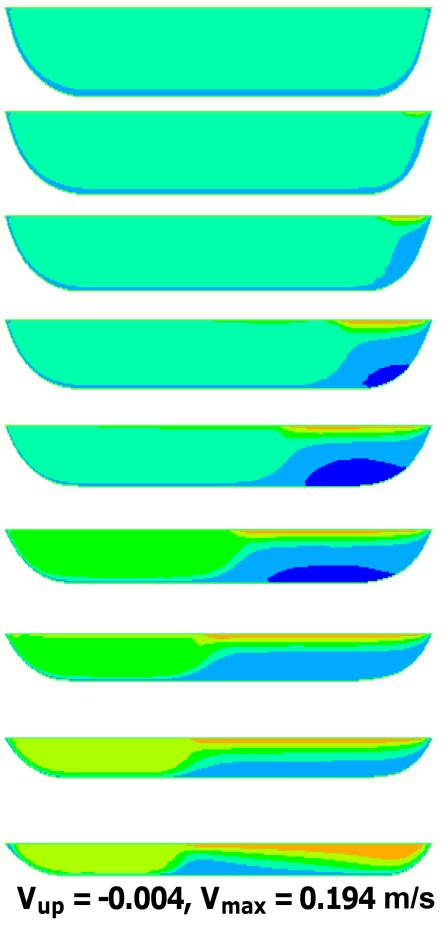

(b)
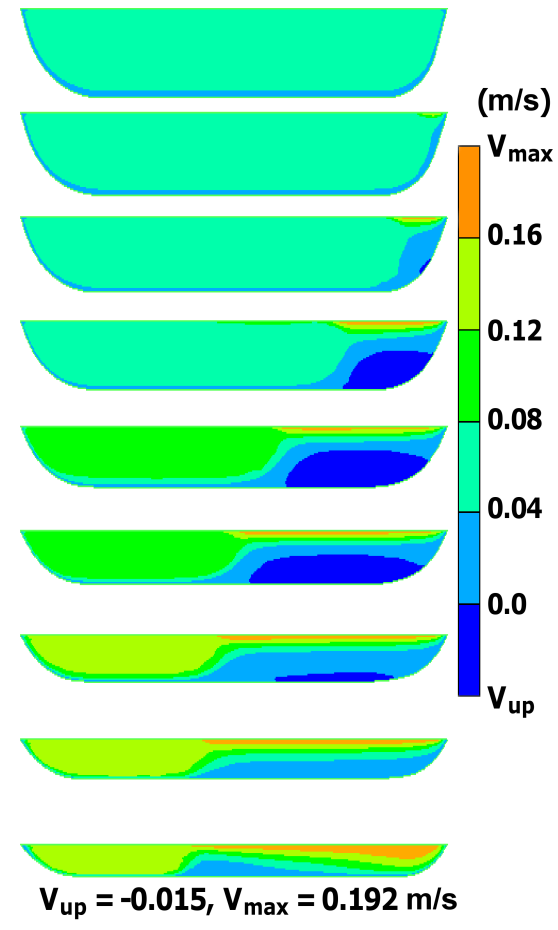

(c)

Figure 3.11: Predicted down-channel velocity distribution in the melting section of the extruder for constant screw temperatures of (a) 230 , (b) 250 , and (c) $270{ }^{\circ} \mathrm{C}$.

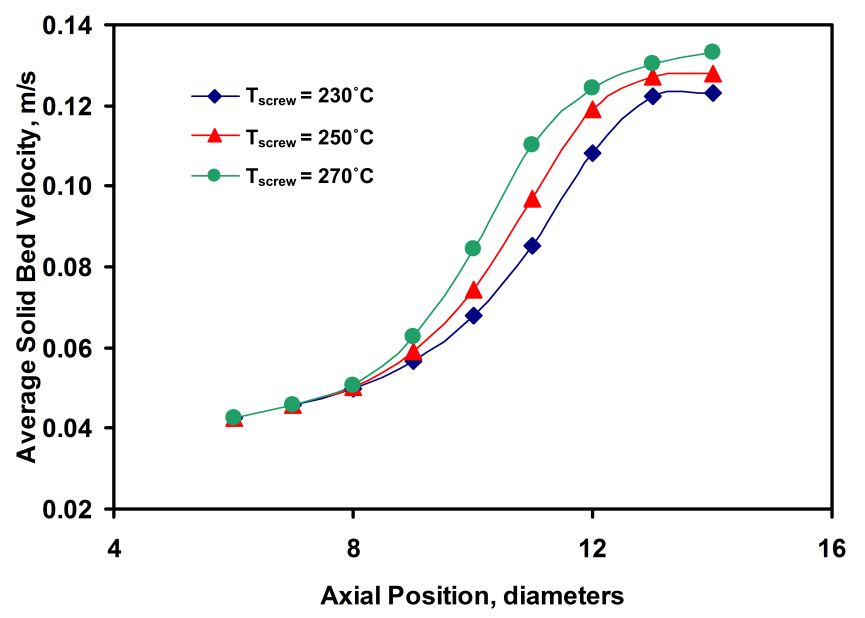

Figure 3.12: Predicted average down-channel velocity of the solid-bed in the melting section of the extruder for screw temperatures of 230,250 , and $270{ }^{\circ} \mathrm{C}$. 


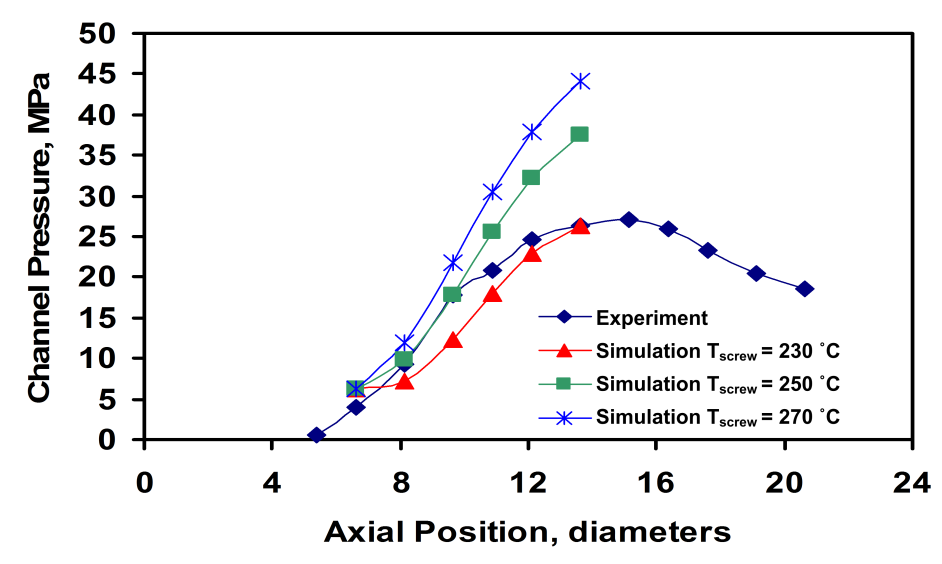

Figure 3.13: Average pressures recorded in experiment and corresponding predictions for different screw temperatures.

the screw channel were calculated. It is important to note here that the pressure values in simulations are obtained up to a constant. Therefore, a constant number can be added to the all pressure values without affecting the velocity and temperature predictions. For the case with a screw temperature of $230{ }^{\circ} \mathrm{C}$, which is compared with the experimental data, the calculated average pressure values were shifted by a constant amount so that the average pressure value calculated at the last pressure transducer location in the compression section of the extruder channel (axial position $=13.6$ diameters) was the same as the corresponding experimental data. For the two simulations with screw temperatures of 250 and $270{ }^{\circ} \mathrm{C}$, the predicted pressure values were shifted by a constant amount such that the pressure value at the entrance of the screw channel is the same as for the simulation with a screw temperature of $230{ }^{\circ} \mathrm{C}$. These average pressure values thus obtained are shown in Fig. 3.13. As shown in Fig. 3.13, the predicted pressure profile for screw temperature of $230{ }^{\circ} \mathrm{C}$, in general, is in good agreement with the corresponding experimental data. It is also noted in Fig. 3.13 that as the screw temperature increases, a higher pressure gradient is obtained along the compression section of the screw channel.

In order to investigate the effect of the screw temperature on flow rate, simulations were also performed with the zero-traction boundary condition specified at the entrance 


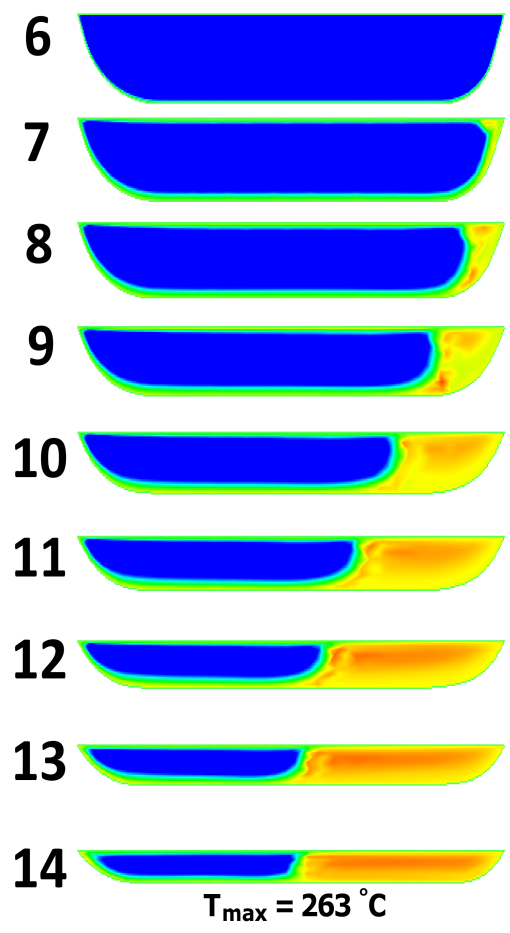

(a)
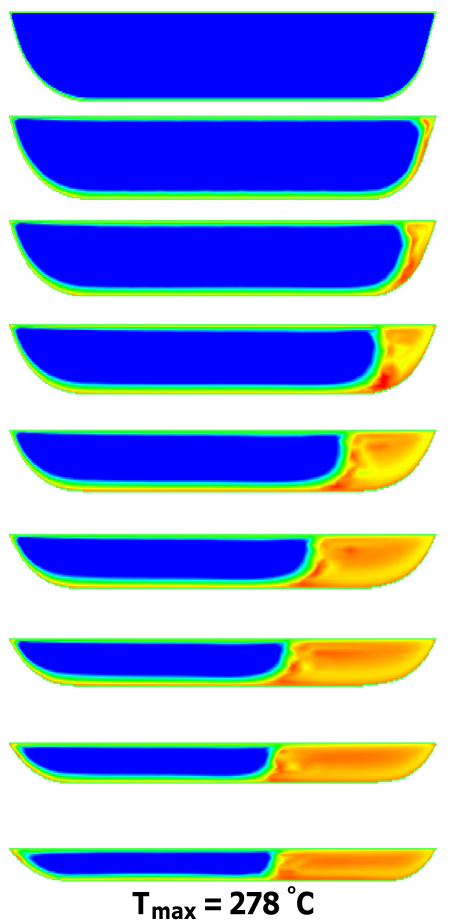

(b)

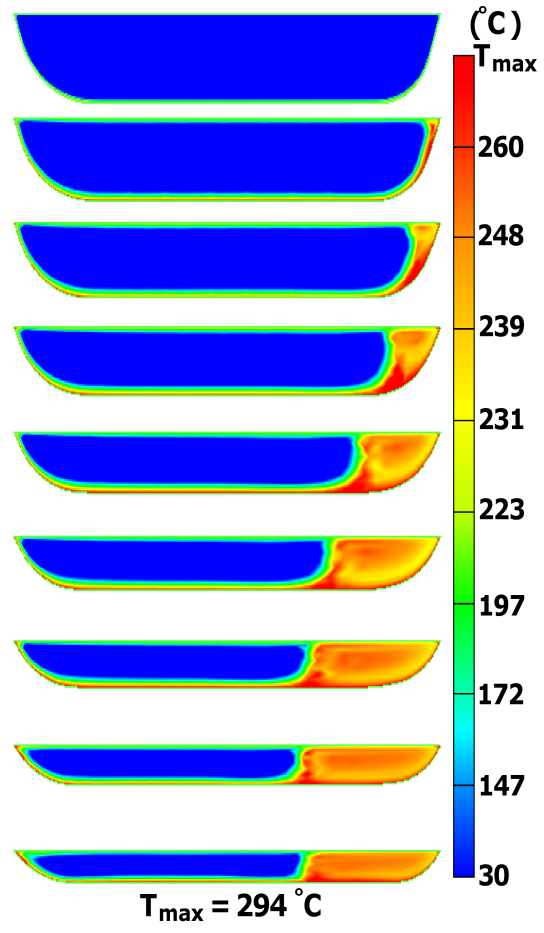

(c)

Figure 3.14: Predicted temperature distribution in the melting section of the extruder for constant screw temperatures of (a) 230 , (b) 250 , and (c) $270{ }^{\circ} \mathrm{C}$ (the zero-traction boundary condition enforced at the entrance of the screw channel).

for constant screw temperatures of 230,250 and $270{ }^{\circ} \mathrm{C}$. The predicted temperature distributions from these simulations are shown in Fig. 3.14. Since the heat conducted into the screw channel increases as the screw temperature is increased, solid fraction in the screw channel was expected to decrease at higher screw temperatures. Against this expectation, it is noted in Fig. 3.14 that the solid fraction in the screw channel increased at higher screw temperature. Since the flow rate was not enforced at the entrance, the predicted flow rate in the channel changed with the screw temperature. The flow rate values obtained from the simulations with zero-traction boundary condition imposed at the entrance were $69.9,85.3$ and $96.9 \mathrm{~kg} / \mathrm{h}$ for screw temperatures of 230,250 and 270 ${ }^{\circ} \mathrm{C}$, respectively. This increase in flow rate for higher screw temperatures is expected 
because an increase in the screw temperature decreases the shear viscosity, and hence, decreases the shear stress, near the screw surface which results in an increase in the flow rate. Since the flow rate is higher at higher screw temperatures, polymer requires smaller time to go through the screw channel as the screw temperature is increased. Because of smaller residence time, the time available for the heat to conduct through the solid bed decreases as the screw temperature is increased. Accordingly, the solid fraction at a fixed screw channel cross-section increases as the screw temperature is increased.

\section{The Effect of Barrel Temperature on Melting}

In contrast to the screw temperature, since the barrel temperature is controlled and maintained at a set value in each of the three zones of an extruder, the barrel temperature is usually assumed to be constant in most modeling and numerical analysis. However, even if the barrel temperature is set to a fixed value in each zone, because of heat conduction in the axial direction [91], the barrel temperature can vary along the screw axis. Moreover, since the thermocouples in the barrel are located away from the inside surface of the barrel, the temperatures measured by the thermocouples can be somewhat different than that at the inside barrel surface.

To analyze the effect of barrel temperature on melting profile, flow in the melting section of the extruder was simulated with barrel temperatures of 230,250 and $270{ }^{\circ} \mathrm{C}$. All other processing conditions were kept the same as those for the basic case. For the three different barrel temperatures, the simulations were first performed by enforcing the flow rate at the entrance of the screw channel to be the same as that measured experimentally. A second set of simulations were then performed for the three constant barrel temperatures using the no-traction boundary condition at the entrance of the screw channel. 
The results for the first set of simulations, with experimentally measured flow rate enforced at the entrance, are given in Fig. 3.15. In Fig. 3.15, for different barrel temperatures there is only minor change in the percentage of the solid polymer at the same cross-section. In particular, the solid fraction at the same cross-section increases slightly with increasing barrel temperature. This slight increase in the solid fraction is also evident in Fig. 3.16, which shows the variation of solid fraction with axial position of the screw. This counter intuitive increase in solid fraction with increasing barrel temperature is because of a decrease in heat generation due to viscous dissipation at higher barrel temperatures. An increase in the barrel temperature increases the temperature of the polymer in the melt film between the barrel and the solid bed. At higher temperatures, viscosity of the polymer in the melt film, and hence, the heat generated due to viscous dissipation, decreases. Besides increasing the polymer temperature in the melt film, in Fig. 3.15, a higher barrel temperature also increases the predicted melt pool temperature at each cross-section.

The simulation results with the zero-traction boundary condition enforced at the channel entrance are shown in Fig. 3.17. The flow rates calculated from these simulations were $69.9,52.6$ and $41.8 \mathrm{~kg} / \mathrm{h}$ for barrel temperatures of 230,250 and $270{ }^{\circ} \mathrm{C}$, respectively. As expected, an increase in barrel temperature resulted in a decrease in flow rate since the shear viscosity, hence, the shear stress, near the barrel surface decreased. This decrease in flow rate with increasing barrel temperature resulted in less solid at the same cross-section because at smaller flow rate it takes longer time for the solid polymer to pass through the screw channel. Therefore, now there is more time available for heat to conduct into the solid bed.

For the first set of simulations with different barrel temperatures and the same flow rate enforced at the entrance, the predicted average pressure values at various locations of the screw channel along with the corresponding experimental data are shown in Fig. 


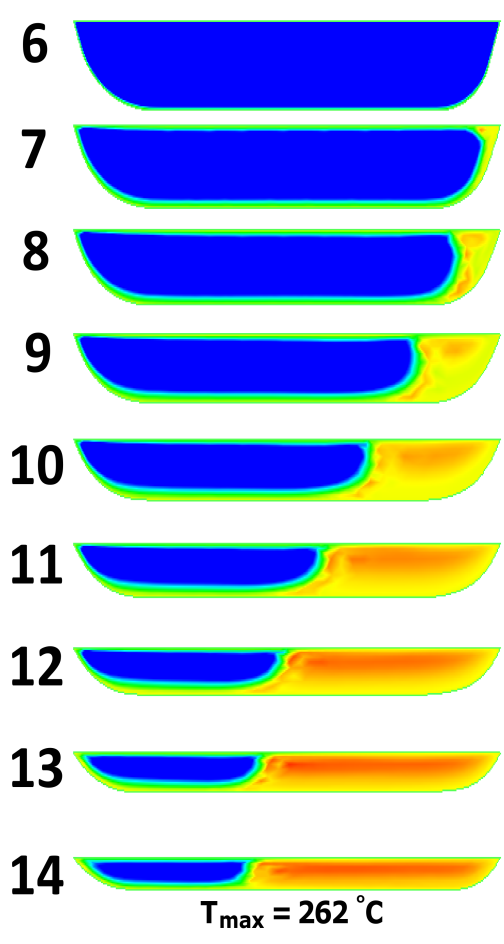

(a)

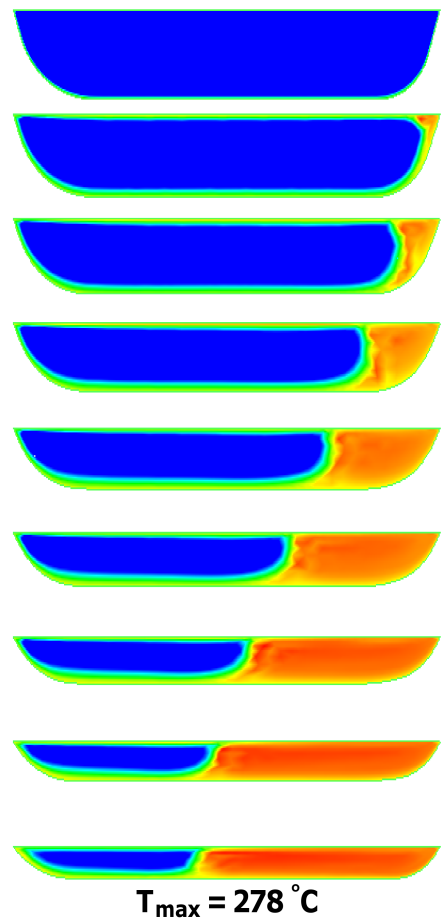

(b)

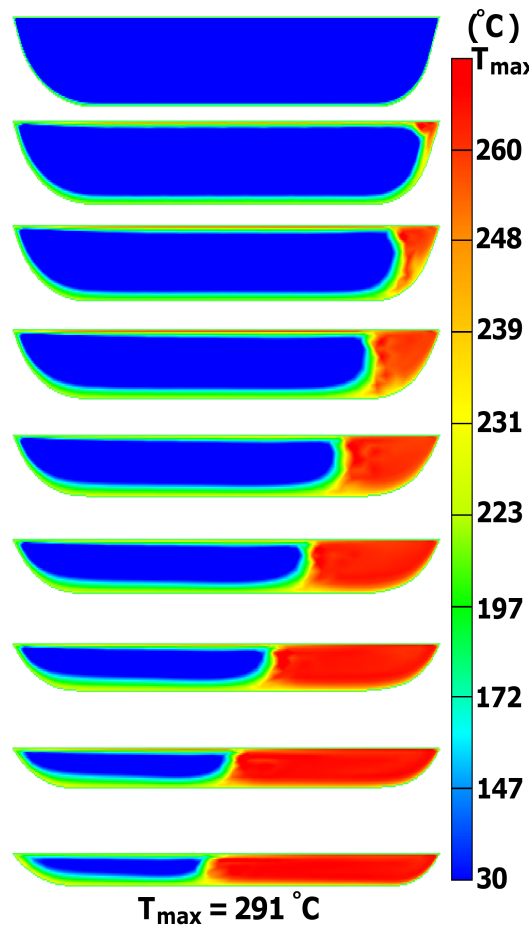

(c)

Figure 3.15: Predicted temperature distribution in the melting section of the extruder for a constant barrel temperature of (a) 230 , (b) 250 , and (c) $270{ }^{\circ} \mathrm{C}$.

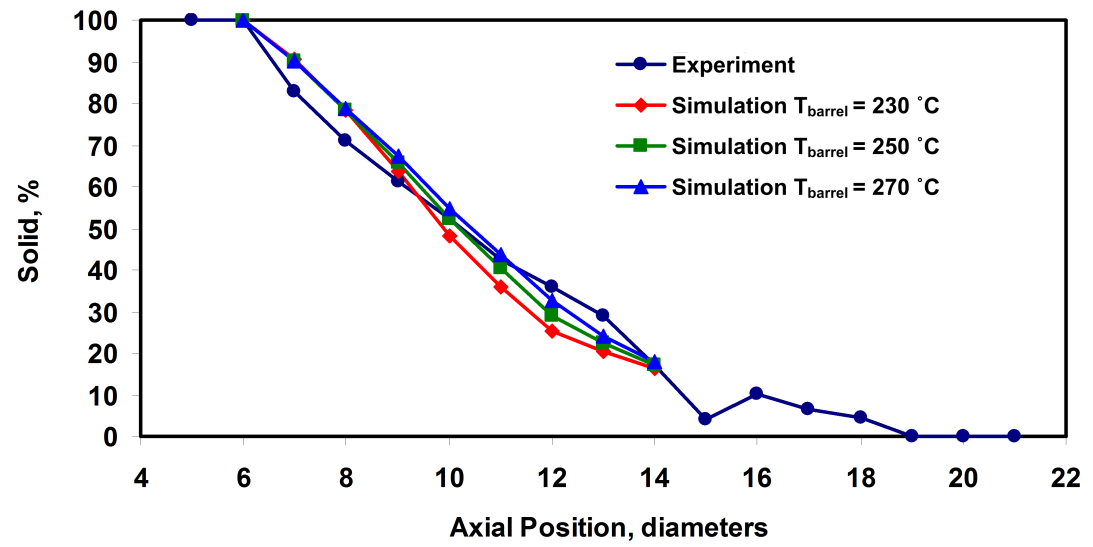

Figure 3.16: Comparison of predicted melting profiles for barrel temperatures of 230, 250, and $270{ }^{\circ} \mathrm{C}$. 


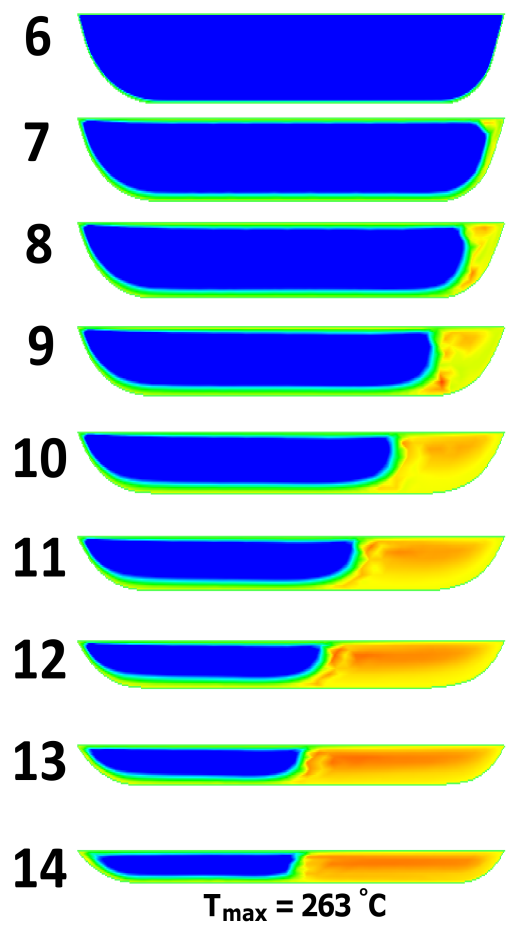

(a)
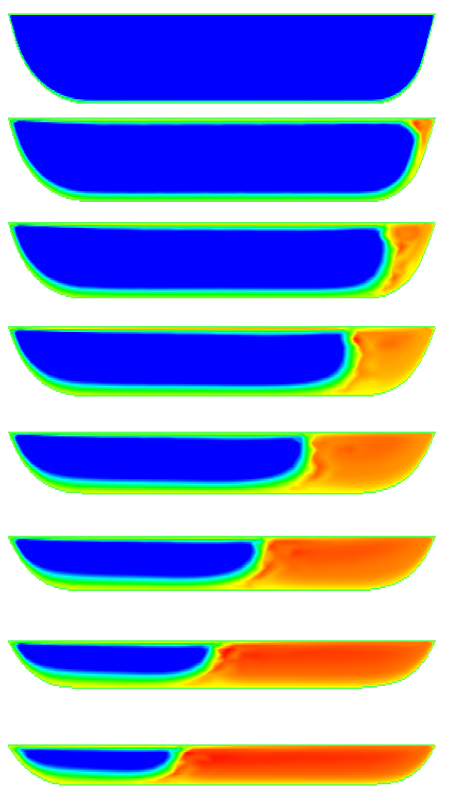

$\mathrm{T}_{\max }=274^{\circ} \mathrm{C}$

(b)

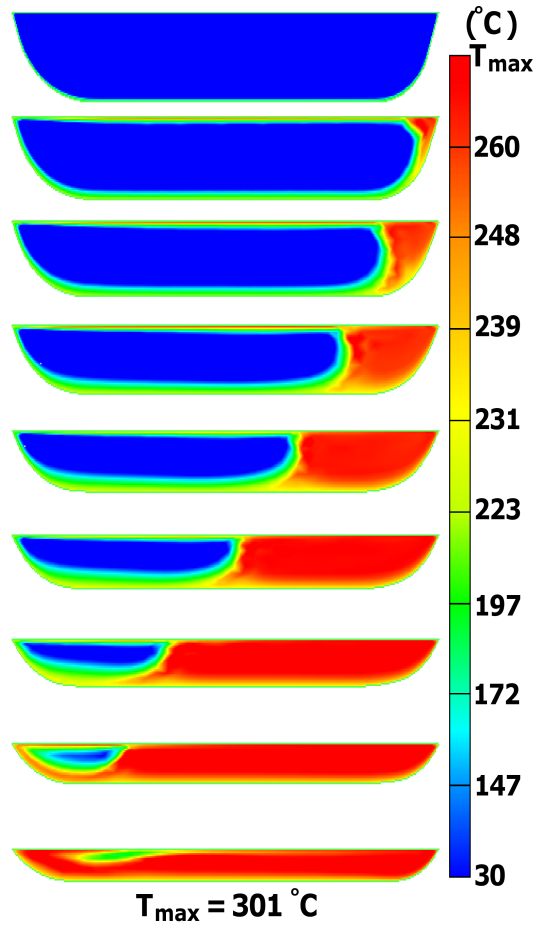

(c)

Figure 3.17: Predicted temperature distribution in the melting section of the extruder for constant barrel temperatures of (a) 230 , (b) 250 , and (c) $270{ }^{\circ} \mathrm{C}$ (the zero-traction boundary condition enforced at the entrance of the screw channel).

3.18. Again, the predicted pressure for the case with barrel temperature of $230{ }^{\circ} \mathrm{C}$, the pressure values were moved by a constant amount to match the pressure at the exit with the corresponding experimental value, and the pressure values for barrel temperatures of 250 and $270{ }^{\circ} \mathrm{C}$ were moved such that pressure at the entrance is the same for all three simulations. It is evident from Fig. 3.18 that with increasing barrel temperature the pressure gradient in the down-channel direction decreases and even becomes negative at higher temperatures. As mentioned in the discussion for the second set of boundary conditions for the barrel temperature, an increase in barrel temperature results in a decrease in flow rate if zero-traction boundary condition is enforced at the entrance of the screw channel. However, when a constant flow rate is imposed at the entrance of 


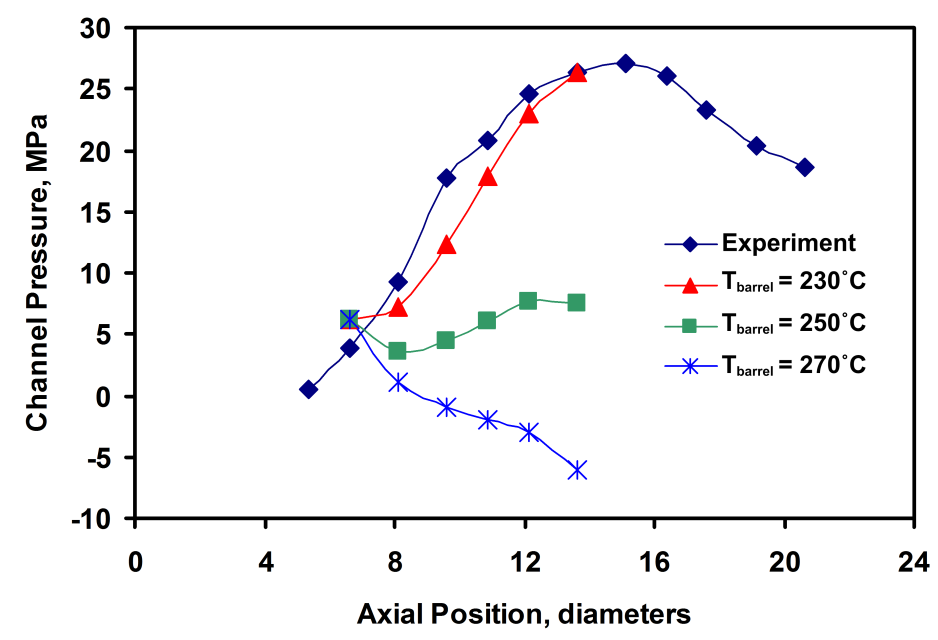

Figure 3.18: Average pressures recorded in experiment and corresponding predictions for different barrel temperatures.

the screw channel, in order to compensate the decrease in the flow rate with increasing barrel temperature, the pressure gradient decreases and even becomes negative as shown in Fig. 3.18. The negative pressure gradient for $T_{\text {barrel }}=270{ }^{\circ} \mathrm{C}$ in Fig. 3.18 suggests that the flow rate enforced at the entrance of the screw channel is too large for a barrel temperature of $270{ }^{\circ} \mathrm{C}$.

\section{The Sensitivity of the Melting Profile to Material Properties}

The effect of various material properties on the melting profile was also investigated in this work. All processing conditions were kept the same as those for the basic case (Fig. 3.2). Also, all other material parameters, except the one whose effect is being investigated, were kept the same. The material properties whose effect on melting of polymers was investigated included thermal conductivity, heat capacity and shear viscosity. In this sensitivity analysis, two different values of thermal conductivity, heat capacity, and shear viscosity were analyzed around the basic case values. For thermal conductivity and heat capacity, 0.9 and 1.1 times the basic case values were used for the simulations whereas 
the value of shear viscosity at the same temperature and the shear rate was doubled and halved compared to the viscosity for the basic case.

First, the sensitivity of the melting profile to the changes in shear viscosity was investigated and the numerical results are shown in Fig. 3.19. It is evident from Fig. 3.19 that as the value of the shear viscosity is increased, less solid is observed at the same cross-section and the temperature of the melt pool increases. The decrease in the solid fraction at the same cross-section is also evident in Fig. 3.20. This decrease in the solid fraction at the same cross-section and the increase in the melt pool temperature were expected because the amount of viscous dissipation increases with an increase in the shear viscosity value. Fig. 3.21 shows the effect of shear viscosity on the predicted pressure profiles. The predicted pressure values were moved by constant values for each of the three simulations, according to the same scheme as that employed for Figs. 3.13 and 3.18. As shown in Fig. 3.21, the predicted pressure gradient in the screw channel increases as the value of the shear viscosity is increased.

The sensitivity of the melting profile to the heat capacity and thermal conductivity was also analyzed. With a $10 \%$ change in the heat capacity and thermal conductivity, no significant change was observed in the melting and pressure profile along the screw channel.

\section{The Effect of Flow Rate on Melting}

For the sensitivity analysis of the polymer melting in a single-screw extruder to the flow rate, the flow rate enforced at the entrance of the channel was changed by 0.75 and 1.25 times the value for the basic case shown in Fig. 3.2. The melting profiles obtained from these numerical simulations are shown in Fig. 3.22 and the solid fraction is shown in Fig. 3.23. It is evident from Figs. 3.22 and 3.23 that an increase in the value of the flow rate enforced at the entrance of the screw channel results in an increase in the solid fraction at 

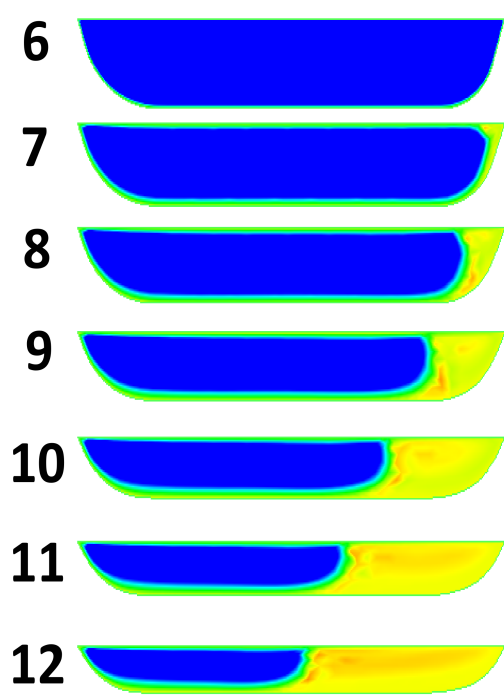

13

14

$\mathrm{T}_{\max }=259^{\circ} \mathrm{C}$

(a)
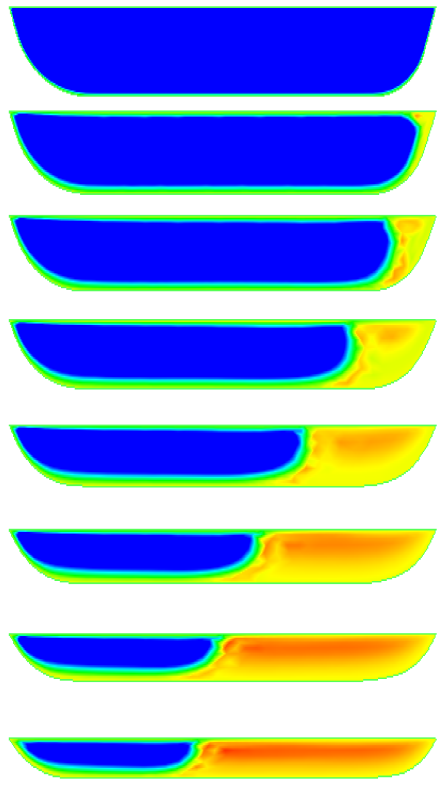

$\mathrm{T}_{\max }=262^{\circ} \mathrm{C}$

(b)

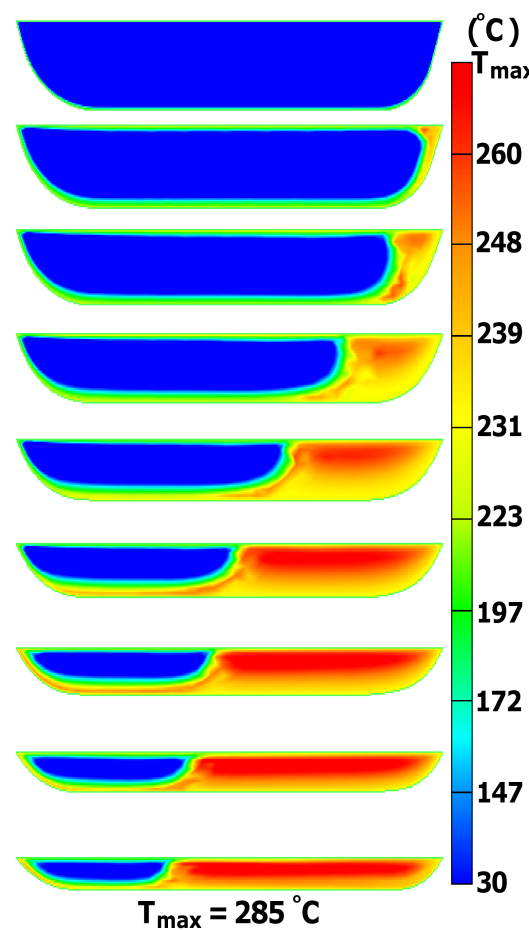

(c)

Figure 3.19: Predicted temperature distribution in the melting section of the extruder when the shear viscosity at the same temperature and shear rate is; (a) reduced by half, (b) the same, and (c) doubled, compared to the basic flow case in Fig. 3.2.

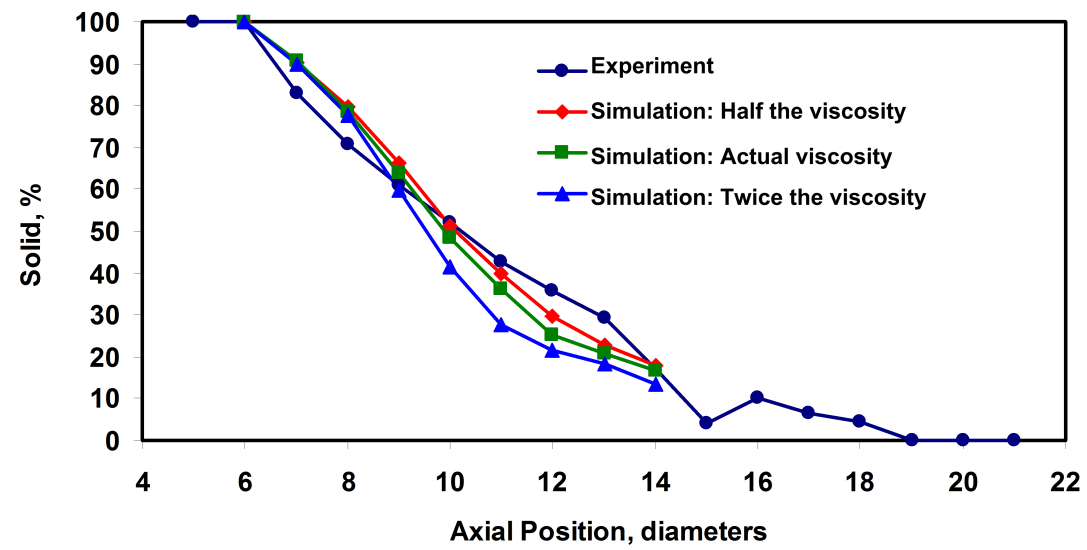

Figure 3.20: Comparison of predicted melting profiles for different values of shear viscosity. 


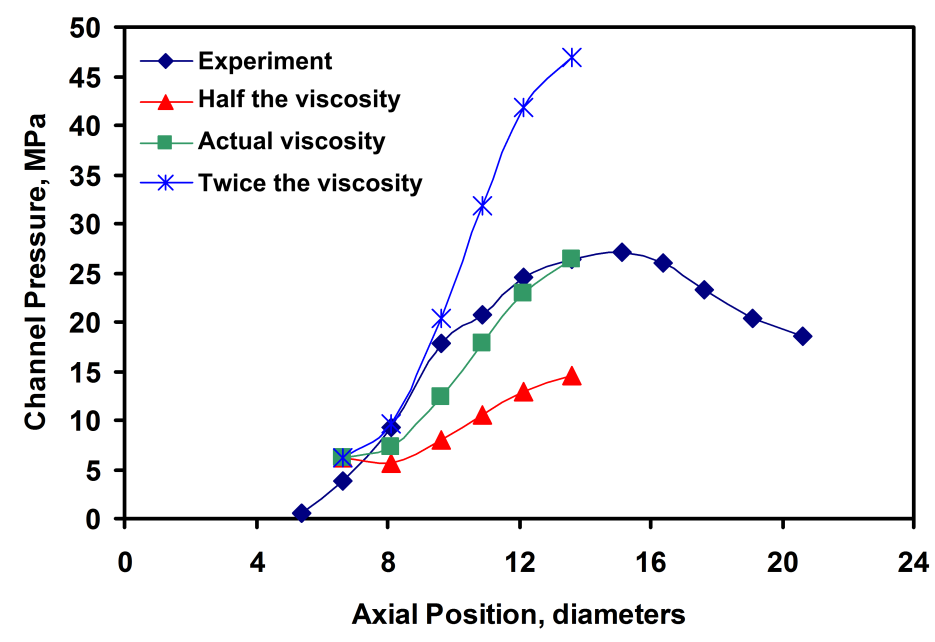

Figure 3.21: Average pressures recorded in the experiment and corresponding predictions for different shear viscosity values.

the same cross-section, and a decrease in the melt pool temperature. As discussed earlier in the effect of the screw temperature section, the reason for the increase in the solid percentage with increasing flow rate is the shorter time required for the solid polymer to pass through the screw channel. Thus, at higher flow rates the time available for heat to conduct into the solid bed is shorter. As expected, the predicted pressure gradient along the screw channel in Fig. 3.24 decreases with an increase in the specified flow rate. The higher pressure increase along the screw channel gives larger back-flow, resulting in a smaller flow rate.

\subsubsection{ABS Resin Results: Other Screws}

The numerical simulations of the polymer melting in a single-screw extruder and the corresponding experimental melting profiles with the four screw configurations other than 6-8-7CR2.8 screw are shown in Figs. $3.25-3.28$. The numerical and experimental results for the 6-8-7CR2.8 screw is already given in Fig. 3.2. Figs. 3.29 and 3.30 compare the solid fractions in the photographs shown in Figs. $2.2-2.7$ with the corresponding 


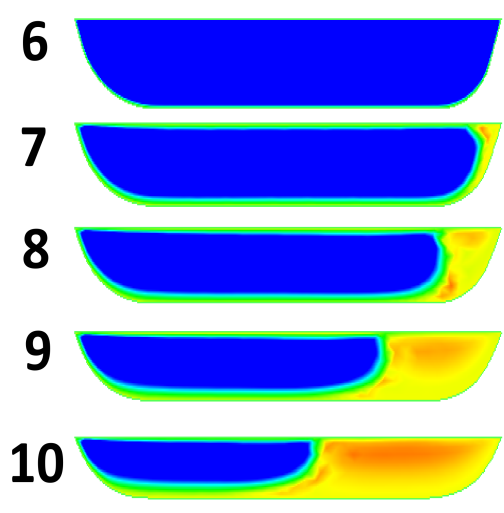

11

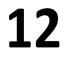

13

14

\section{$T_{\max }=276^{\circ} \mathrm{C}$}

(a)
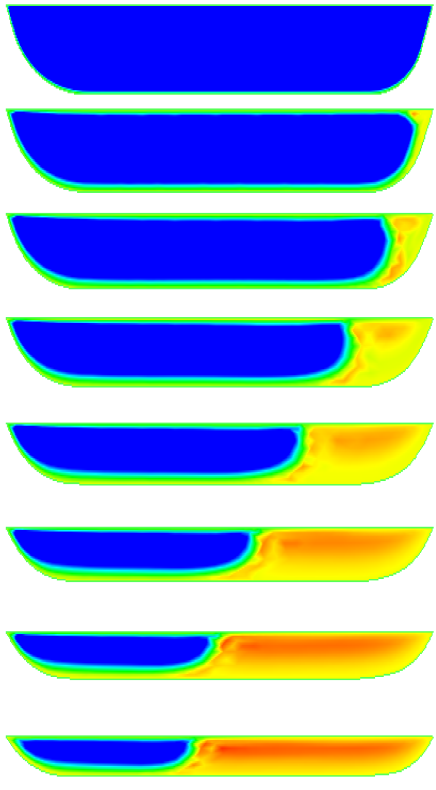

$\mathrm{T}_{\max }=262^{\circ} \mathrm{C}$

(b)

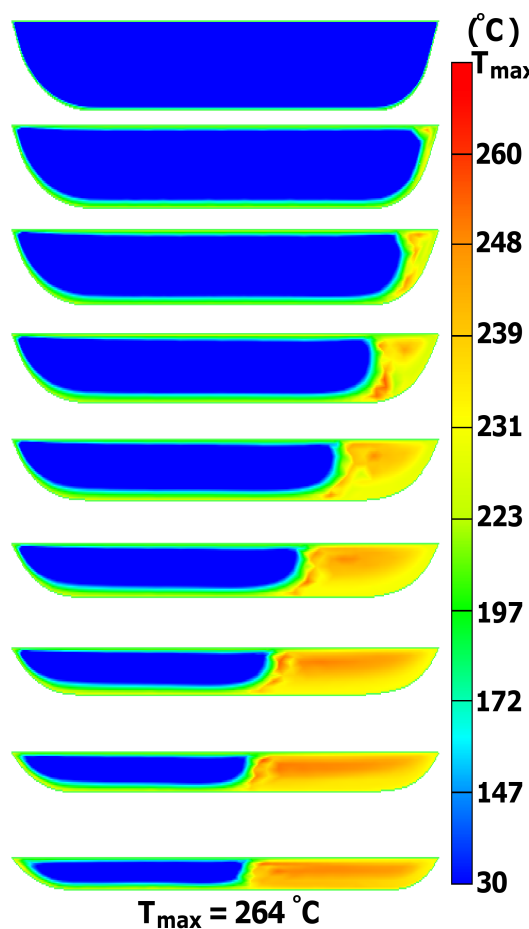

(c)

Figure 3.22: Predicted temperature distribution in the melting section of the extruder for flow rates of (a) 0.75 times, (b) 1.00 times, and (c) 1.25 times the basic case flow rate.

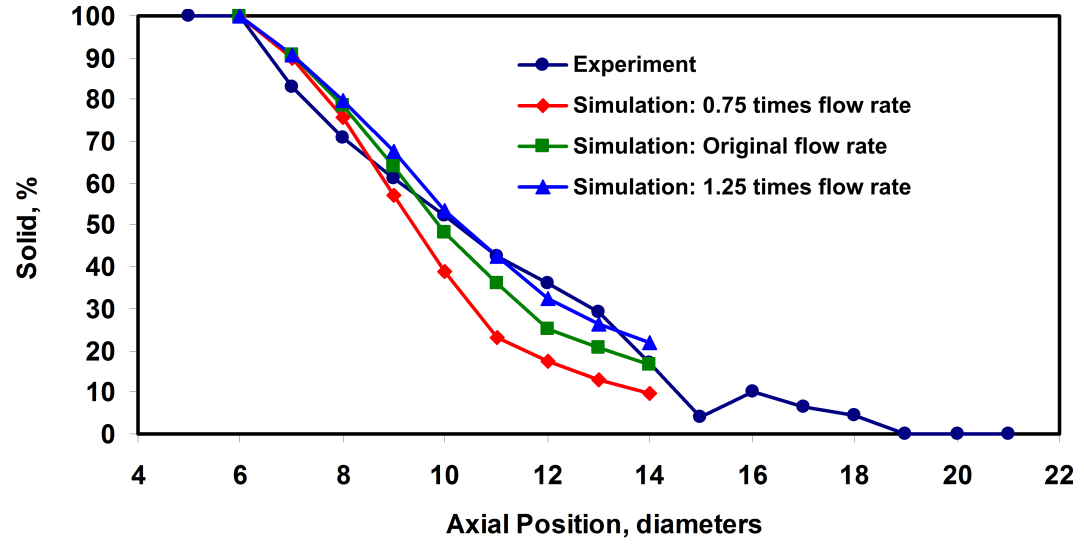

Figure 3.23: Comparison of predicted melting profiles for different values of flow rate. 


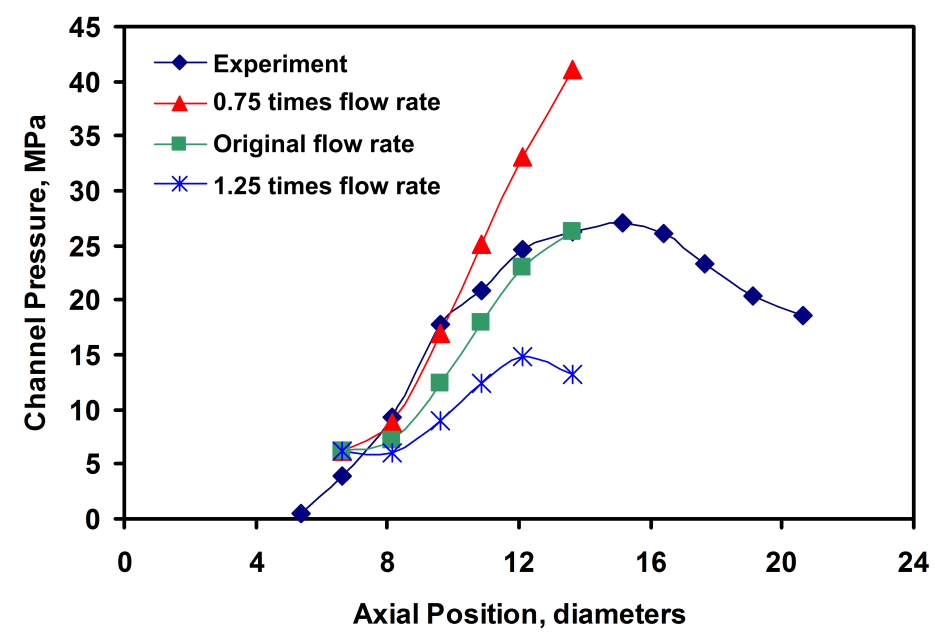

Figure 3.24: Average pressures recorded in the experiment and corresponding predictions for different flow rate values.

predictions in the simulation results presented in Figs. 3.2 and $3.25-3.28$. Equation 2.4 was used to calculate the solid fraction plotted in Figs. 3.29 and 3.30. As shown in Figs. 3.29 and 3.30, in general, the solid fraction along the screw channel in experiments is in good agreement with the numerical predictions. For the 6-8-7CR2.4 and the 6-8-7CR3.2 screws, for the first few diameters, the numerical simulations had a higher solid fraction than that in experiments. One of the possible reasons for this discrepancy is that the experimental data had a small melt pool at the beginning of the compression section of these two screws; however, in the simulations it was assumed that the polymer at the beginning of the compression section was at $30^{\circ} \mathrm{C}$, that is, the polymer was fully solid. Further down the screw channel, the numerical simulations for 6-8-7CR2.8 and 6-8-7CR3.2 screws indicated faster melting than the experimental data. As mentioned earlier, this faster melting rate may be due to the assumption of a constant heat capacity of polymer in numerical simulations for both solid and liquid states.

Figs. 3.31 and 3.32 show the predicted average pressure at various cross-sections of the screw channel and the corresponding experimental data for the five different screw 

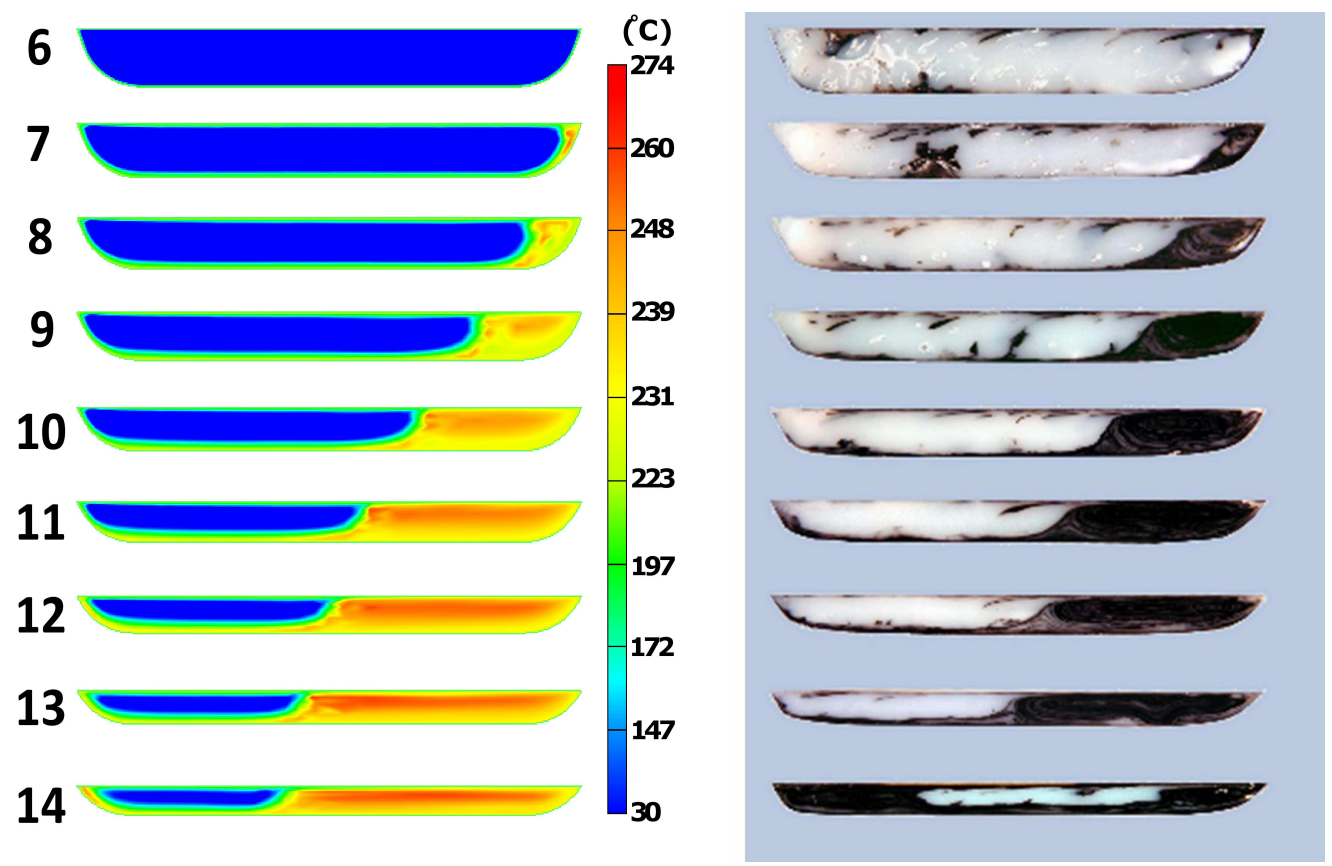

Figure 3.25: Predicted temperature distribution for the 6-8-7CR2.0 screw (left) and the corresponding experimental data from Fig. 2.2 (right).
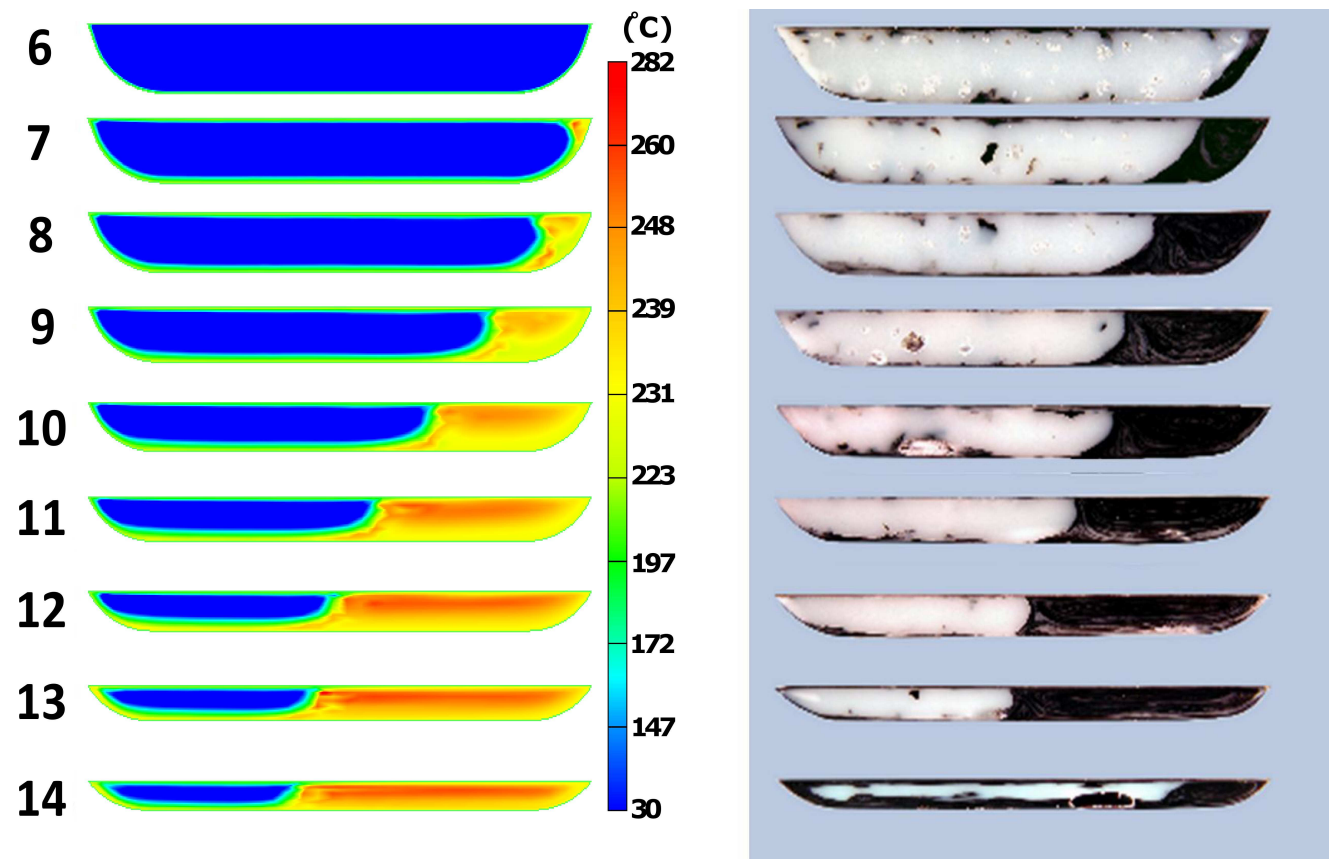

Figure 3.26: Predicted temperature distribution for the 6-8-7CR2.4 screw (left) and the corresponding experimental data from Fig. 2.3 (right). 

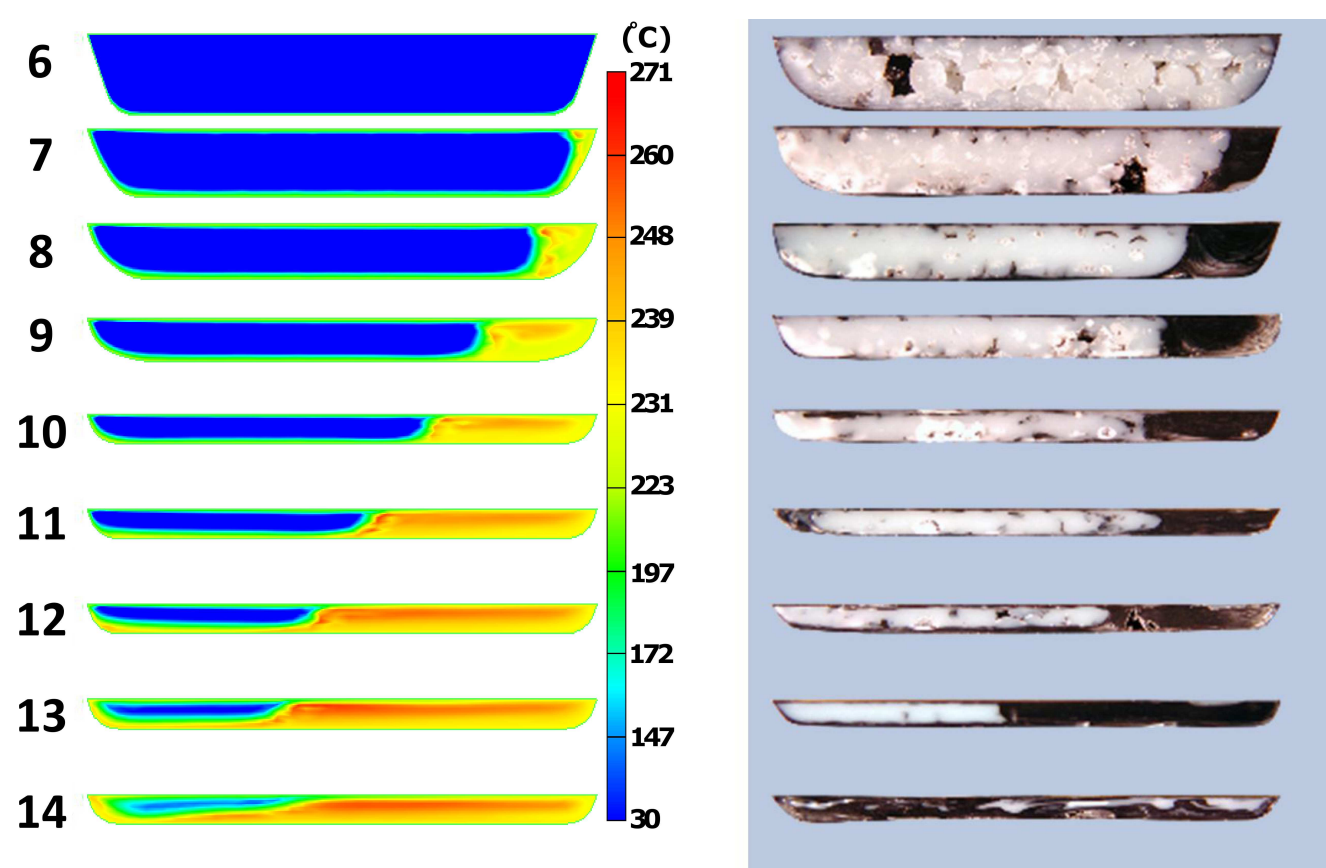

Figure 3.27: Predicted temperature distribution for the 6-4-11CR2.8 screw (left) and the corresponding experimental data from Fig. 2.5 (right).
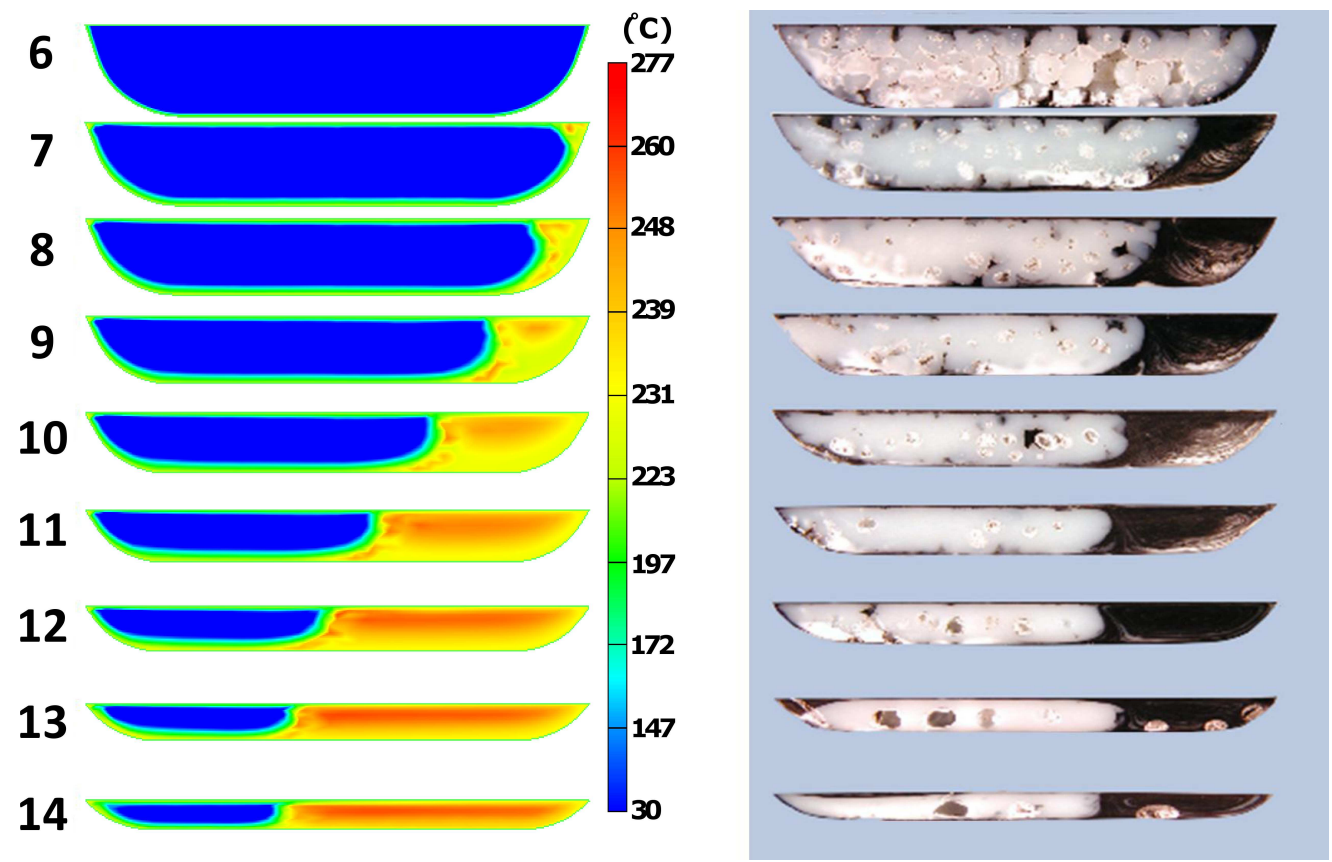

Figure 3.28: Predicted temperature distribution for the 6-8-7CR3.2 screw and the corresponding experimental data from Fig. 2.6 (right). 


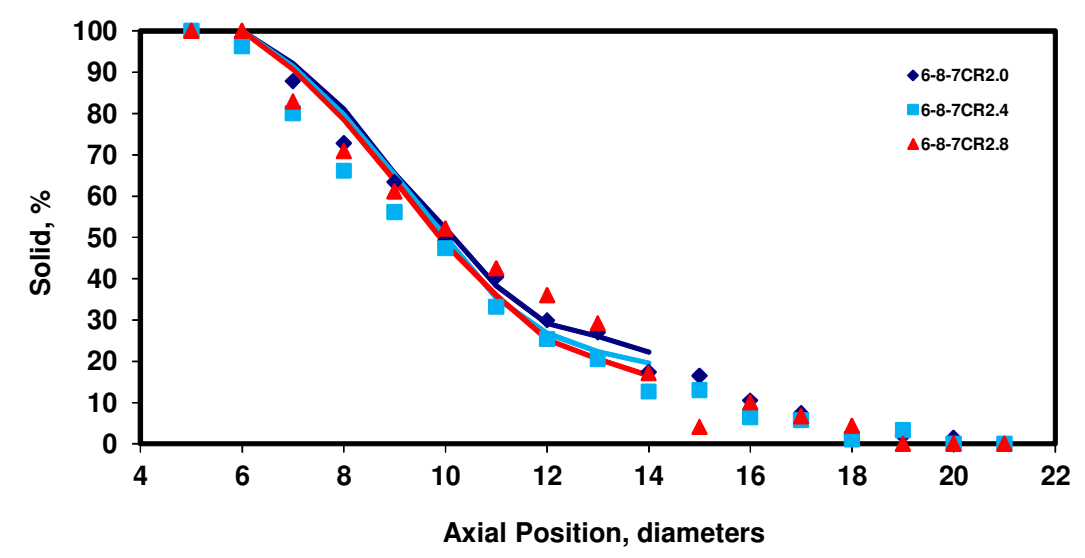

Figure 3.29: Comparison of experimental (filled circles) and numerical (lines) melting profile for 6-8-7CR2.0, 6-8-7CR2.4, and 6-8-7CR2.8 screws.

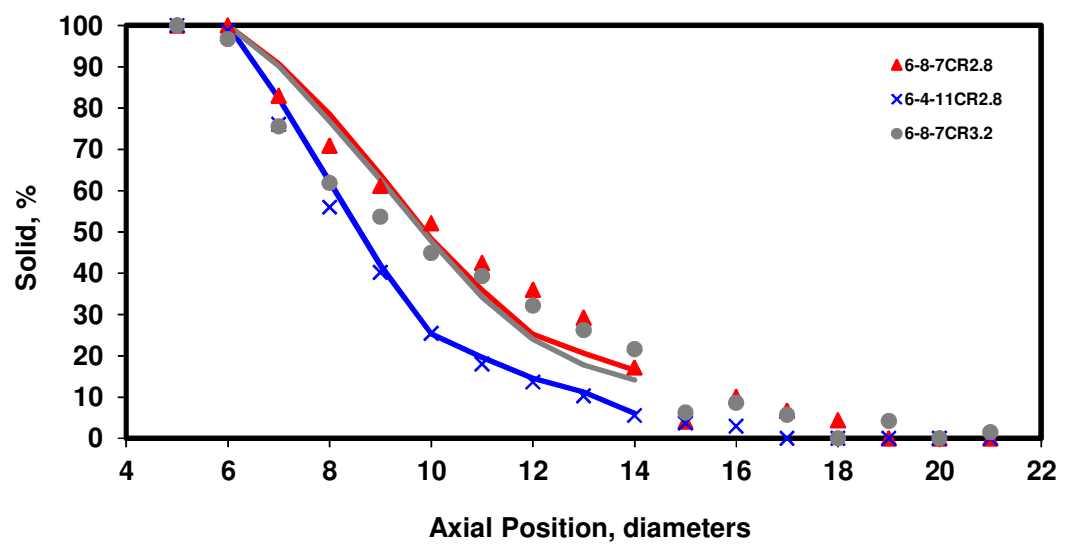

Figure 3.30: Comparison of experimental (filled circles) and numerical (lines) melting profile for 6-8-7CR2.8, 6-4-11CR2.8, and 6-8-7CR3.2 screws. 


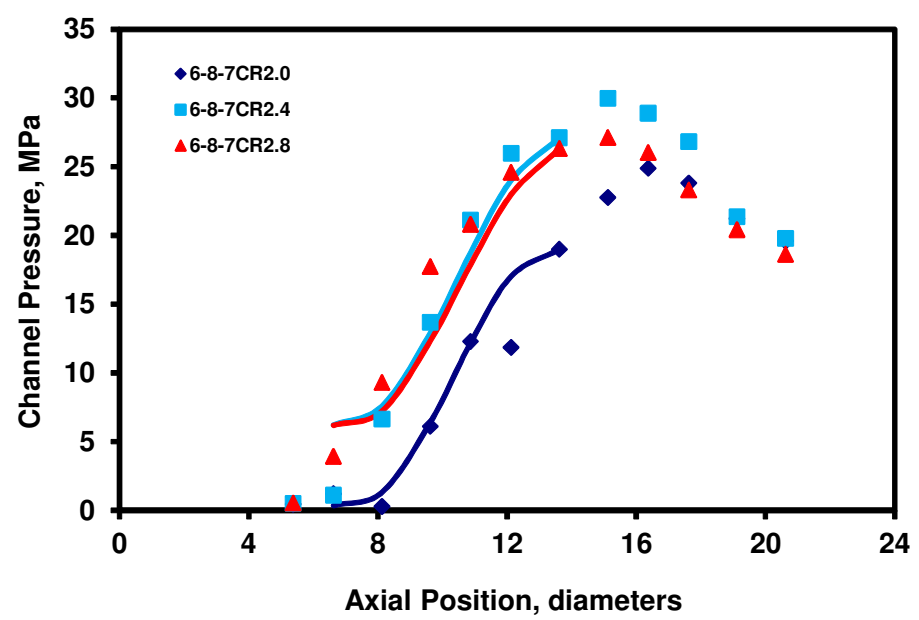

Figure 3.31: Average pressures recorded in the experiments (filled circles) and corresponding predictions (lines) for different screw configurations.

configurations. As mentioned earlier, in the experiments, pressure transducers were tap mounted on the inside surface of the extruder barrel. As the screw rotates inside the barrel, these pressure transducers measure a time dependent pressure profile at an axial cross-section of the screw channel. In simulations, the average pressure values across the same axial cross-section of the screw channel were calculated. The calculated average pressure values were then shifted by a constant amount so that the average pressure value calculated at the last pressure transducer location in the simulated portion of the extruder channel was the same as the corresponding experimental data. These average pressure values are shown in Figs. 3.31 and 3.32. In general, the predicted pressure profiles are in good agreement with the corresponding experimental data. It should also be noted that for a fixed axial length of compression section, as the compression ratio was increased, the pressure value at the same location of the screw channel was typically higher. 


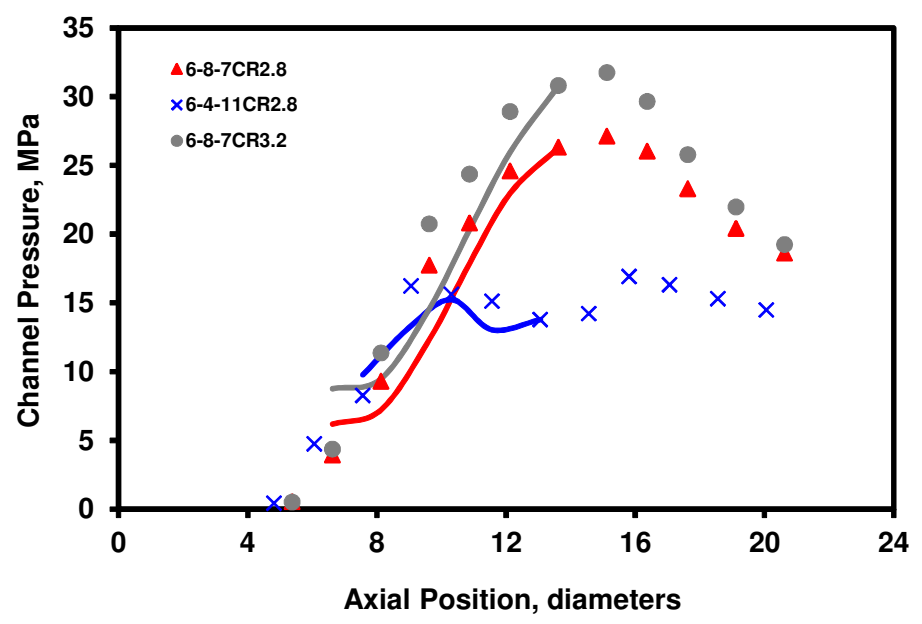

Figure 3.32: Average pressures recorded in the experiments (filled circles) and corresponding predictions (lines) for different screw configurations.

\subsubsection{LDPE Resin Results}

As discussed in Section 2.1, Maddock screw-freezing experiment was performed for a semi-crystalline polymer (LDPE) using a 6-8-7 screw with a compression ratio of 2.8. The melting profile data from this experiment was shown in Fig. 2.7. The numerical simulation for the LDPE resin with the boundary conditions discussed in Section 3.4 was also performed. That is, the boundary conditions were - constant barrel and screw temperature of $175{ }^{\circ} \mathrm{C}$, and flow rate of $45.6 \mathrm{~kg} / \mathrm{h}$. The temperature distributions at various cross-sections of the screw channel obtained from the numerical simulation along with the corresponding experimental data is shown in Fig. 3.33. As discussed in Section 2.3.1, the melting temperature of the LDPE resin is $111{ }^{\circ} \mathrm{C}$ and below the melting temperature of the LDPE resin, the polymer was assumed to be in solid state. Therefore, the region below $111{ }^{\circ} \mathrm{C}$, shown with blue color in Fig. 3.33 is solid polymer, whereas other colors show the temperature of the polymer melt.

Fig. 3.34 compares the solid fractions in the photograph of the experimental data and the plot of the corresponding numerical result presented in Fig. 3.33. Equation 

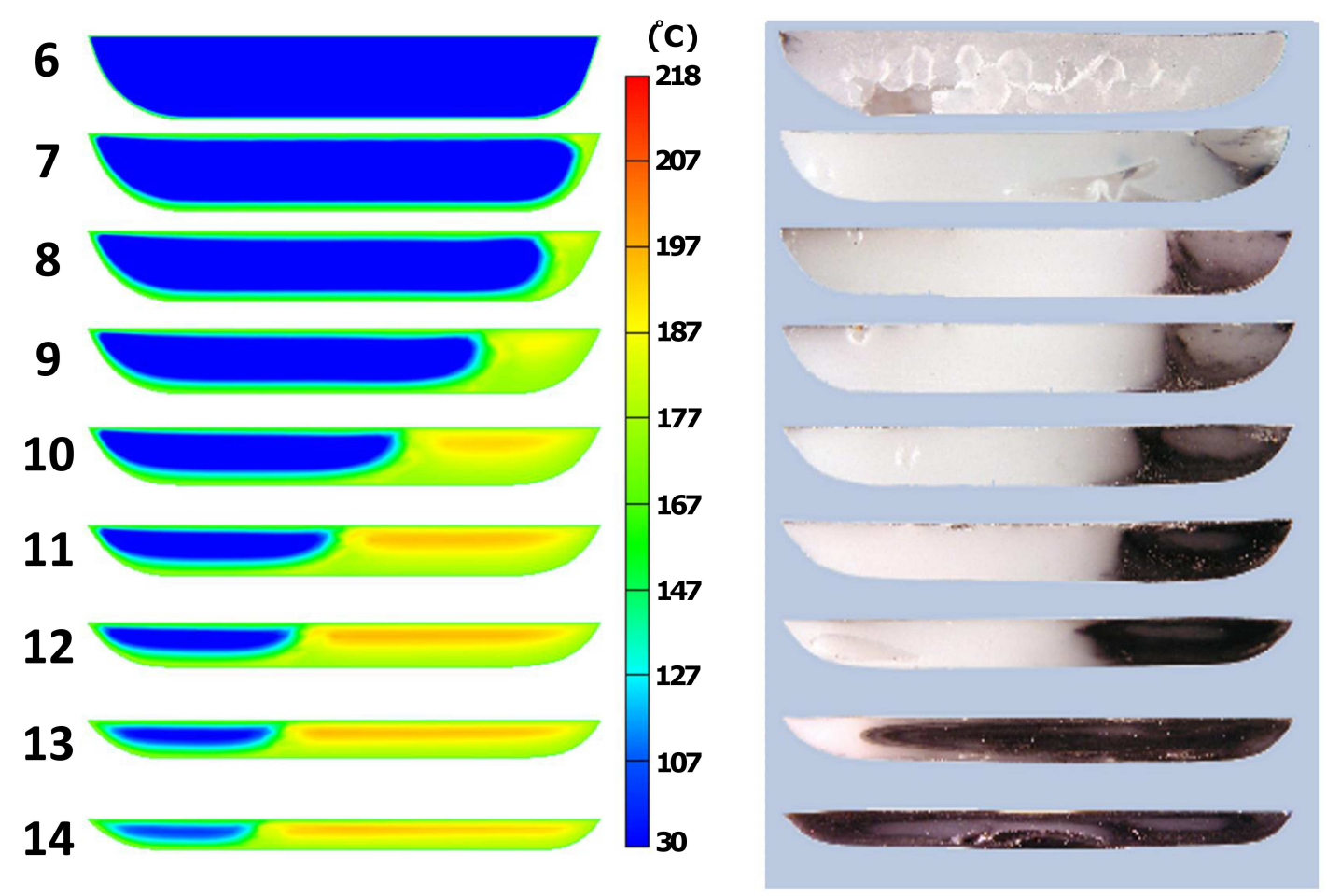

Figure 3.33: Predicted temperature distribution in the melting section of the extruder for the LDPE resin (left) and the corresponding experimental data from Fig. 2.7 (right).

2.4 was used to calculate the solid fractions plotted in Fig. 3.34. As shown in Figs. 3.33 and 3.34 , the predictions from the numerical simulation indicated a slightly faster melting rate compared to the experimental data. The assumption of a constant heat capacity of polymer in numerical simulations for both solid and liquid states is believed to contribute to the difference in the melting rate since the temperature dependence of the heat capacity of the semi-crystalline polymers is known to exhibit a localized peak near the melting point.

Fig. 3.35 shows the predicted average pressure at various cross-sections of the screw channel and the corresponding experimental data for the LDPE resin. As mentioned earlier, in the experiments, pressure transducers were tap mounted on the inside surface of the extruder barrel. As the screw rotates inside the barrel, these pressure transducers 


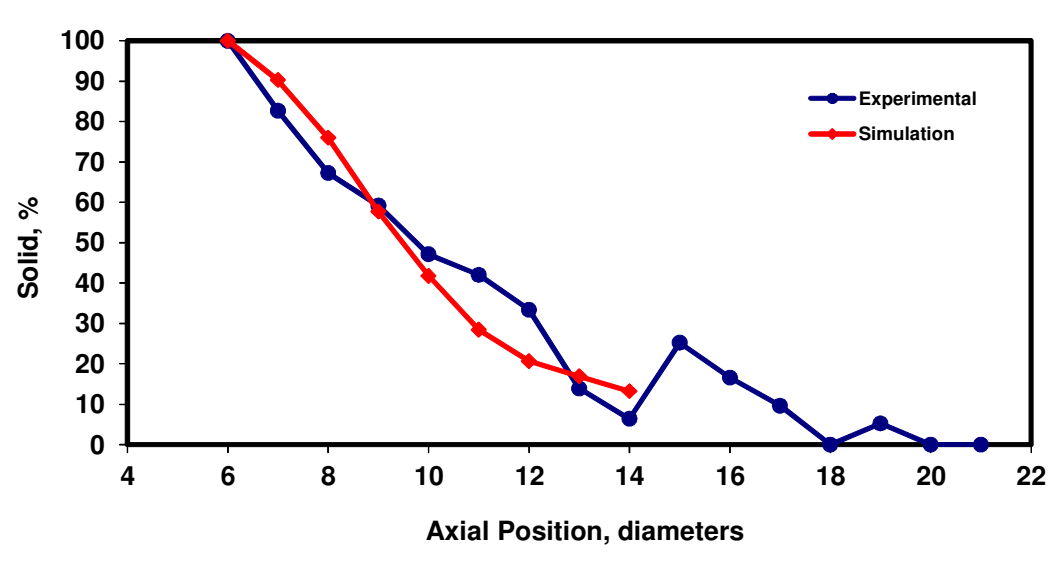

Figure 3.34: Comparison of experimental and numerical melting profile for the LDPE resin.

measure a time dependent pressure profile at an axial cross-section of the screw channel. In the numerical simulation of the LDPE resin, the average pressure values across the same axial cross-section of the screw channel were calculated. The calculated average pressure values were then shifted by a constant amount so that the average pressure value calculated at the first pressure transducer location in the simulated portion of the extruder channel was the same as the corresponding experimental data. These average pressure values are shown in Fig. 3.35. As shown in Fig. 3.35, the predicted pressure gradient along the screw channel is higher compared to the corresponding experimental data.

The sensitivity of melting profile to screw temperature and shear viscosity is examined for the ABS resin in Section 3.6.1. In Section 3.6.1, the results shown in Fig. 3.13 indicated that the predicted pressure value at the same location of the screw channel was lower when a lower temperature was specified at the screw surface. Fig. 3.21 showed that a smaller value of shear viscosity also reduces the predicted pressure in the screw channel. Therefore, a decrease in the temperature specified at the screw surface or a reduction in the shear viscosity is expected to improve the agreement between the experimental data and the corresponding predictions of pressure in the screw channel for the LDPE resin. It 


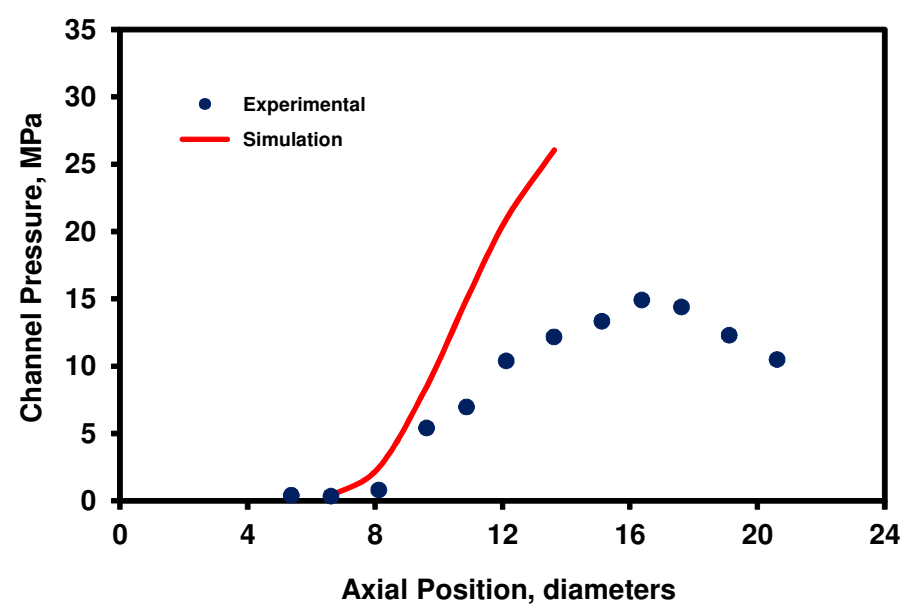

Figure 3.35: Average pressures recorded in the experiment (filled circles) and corresponding prediction (line) for the LDPE resin.

should be noted that a change in the specified screw temperature or the shear viscosity in numerical simulations is also expected to affect the predicted melting profile along the screw channel shown in Figs. 3.33 and 3.34. For the ABS resin, Figs. 3.10 and 3.20 showed that the percentage of the solid polymer at the same cross-section increased with a decrease in the temperature specified at the screw surface and also with a decrease in the shear viscosity. Therefore, with a decrease in the specified screw surface temperature or a decrease in the specified shear viscosity, the numerical predictions for the melting profile of the LDPE resin is also expected to have a better agreement with the corresponding experimental results. 


\section{Chapter 4}

\section{Optimization of a Screw Geometry in}

\section{a Single-screw Extruder}

The goal in design of a single-screw extruder is to have the highest output possible with a good quality of polymer melt at the extrudate. Various design parameters such as barrel temperatures at different zones, screw speed, screw geometry parameters can be adjusted to provide a higher output rate. However, the optimization of all design parameters in a single-screw extruder is computationally time consuming and was not attempted with the computational resources that were available to us during the course of this work. Due to this reason, only two screw geometry parameters, namely, lead (pitch) and the depth of the screw, were optimized in this work.

At each iteration of a gradient based optimization algorithm, in general, the value of the objective function and the gradient of the objective function with respect to design parameters are needed. In order to calculate the objective function value and gradient, in

this work, the generation of the finite element mesh for the screw channel (flow domain) is needed for given values of design parameters. Since the values of the design parameters are changing at each iteration step in the optimization algorithm, the flow domain mesh has to be regenerated for each iteration in the optimization algorithm. This regeneration of the finite element mesh for the flow domain at each iteration is complex when the assumption of unwound channel that was described in Section 3.4 is used for the numerical simulations. Instead, a mesh partitioning technique [92-94] that eliminates the complexity of the flow 
domain mesh regeneration is used in this research. In this technique, a finite element mesh in the barrel and on the screw surface is needed. The barrel volume is represented by linear 3-D tetrahedral elements whereas the surface of the screw is represented by linear triangular finite elements (Fig. 4.1). These linear triangular finite elements on the screw surface are used to partition the mesh of 3-D tetrahedral elements of the barrel to obtain the flow domain mesh. This flow domain mesh is used to simulate the flow of the polymer in the single-screw extruder channel which is needed to calculate the value and the gradient of the objective function. For the simulation of the flow using the mesh partitioning technique, the flow equations are the same as those discussed in Section 3.2. Since the calculation of the objective function value and the objective function gradient is needed several times for the optimization algorithm, the computation time for these calculations should be small. As discussed in Section 3.5, the computation time for one simulation of a two-phase polymer flow was 16 hours, which is not reasonable for an optimization algorithm. Due to this reason, the design parameters (lead and depth of a screw) were optimized only in the metering section of a single-screw extruder where the polymer was assumed to be all liquid at the entrance of the channel. The axial length of the barrel was fixed at seven times the diameter of the screw.

Using the mesh partitioning technique [92-94], the finite element mesh representing the barrel remains the same for each iteration in an optimization algorithm. However, the mesh for the screw has to be regenerated according to the new values of the design parameters at that iteration as discussed earlier. Thus, an automatic generation of the screw surface mesh was needed for the optimization code. For this purpose, a screw mesh generator code was developed. In this mesh generator, for a given set of screw geometry parameters such as screw diameter, screw channel depth in the solid conveying and metering sections, screw pitch, flight width, axial length of screw zones, and flight radii, a triangular finite element mesh of the screw surface was generated. An example 


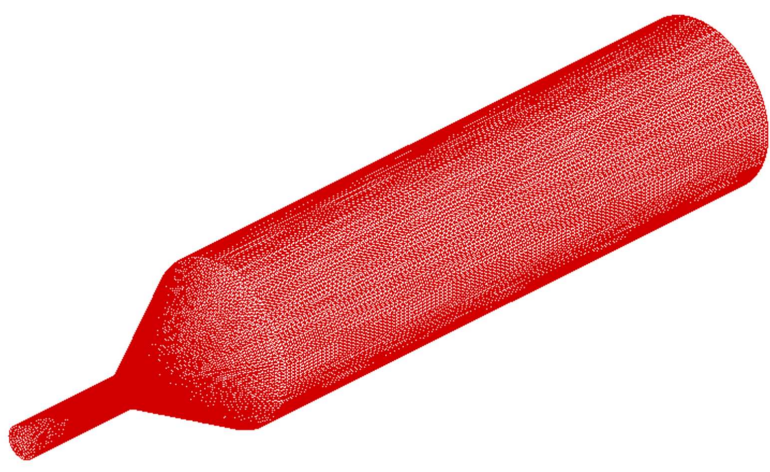

(a)

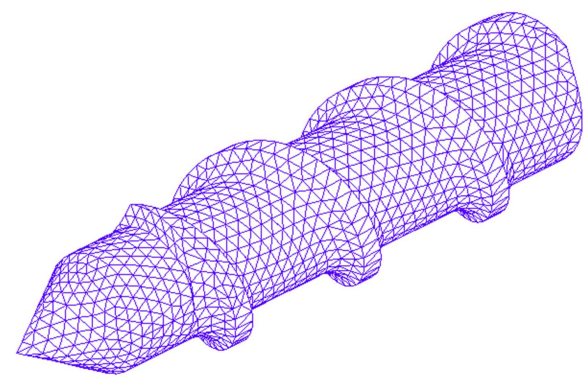

(b)

Figure 4.1: (a) Finite element mesh of tetrahedral elements representing the barrel geometry (b) Finite element mesh of triangular elements representing the surface of the screw.

of the screw surface mesh generated using this code is shown in Fig 4.2. In the process of the mesh generation, first, the code generates the information for the screw channel profile using the given values of the screw geometry parameters. This information is used to discretize the screw channel profile with scattered nodes. These nodes generated along the screw channel profile are shown in Fig 4.2 (b). The distance between each node was determined by the user supplied element length. Later, each node generated along the screw channel profile was used as the start point of a helical curve. The axis of these helical curves coincides with the axis of the screw. These helical curves were then used to generate nodes that are equally distributed along the helical curves. After generating all nodes, the element connectivity between these nodes was established.

In the next section, the formulation of the optimization problem is described. Later, the optimization code algorithm followed by the results obtained using this algorithm are given. 


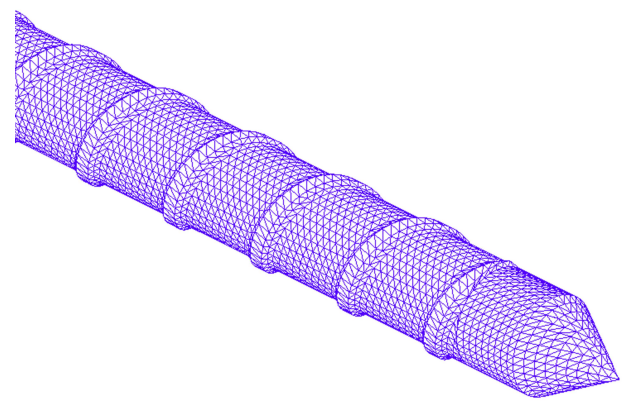

(a)

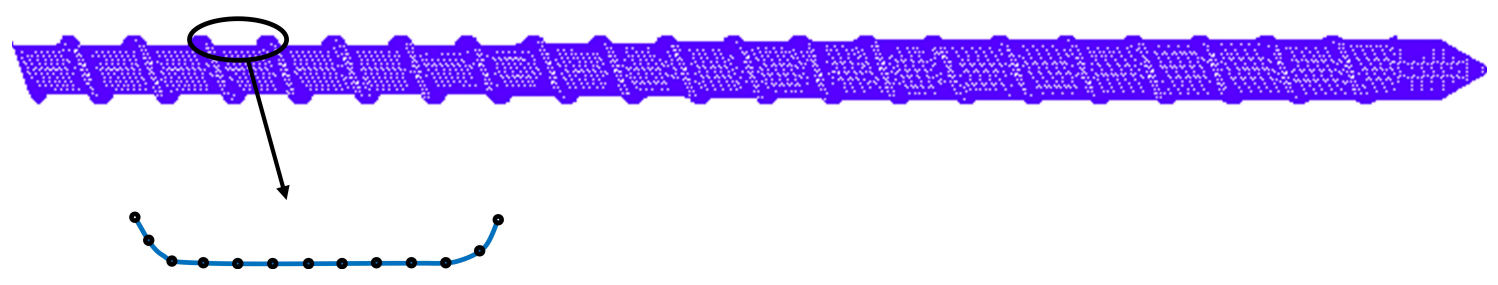

(b)

Figure 4.2: The triangular screw mesh generated using the screw mesh generator code (a) Last 5 diameters of a screw (b) Axial view of the full screw and the discretized screw channel profile.

\subsection{Formulation of the Optimization Problem}

The purpose of the optimization algorithm was to have the highest output rate possible without exceeding the specified temperature at the exit of the extruder. The problem of obtaining the highest output rate possible without excessively increasing the exit temperature can be formulated as a constraint optimization problem. There are two main factors affecting the output rate in a single-screw extruder. These factors are the pressure gradient along the screw channel and the drag force resulting from the barrel surface and rotating screw surface. It should be noted here that for the numerical simulations of polymer flow, the output rate (flow rate) and screw speed has to be specified. Due to this reason, the effect of the pressure gradient along the metering section of an extruder 
on the flow rate should be minimized in the optimization algorithm. With a decrease in the effect of pressure gradient on flow rate, the flow resulting from the drag force (drag flow) increases since a constant flow rate is specified in the numerical simulations. This increase in drag flow results in an increase in the output rate since the pressure gradient in the metering section of a single-screw extruder can be controlled by other means. In order to decrease the pressure gradient in the metering section of a single-screw extruder, the pressure at the entrance of the metering section should be minimized since the pressure at the exit is set to a constant in the numerical simulations. Thus, the optimization problem is formulated as follows:

$$
\min _{\boldsymbol{x}} f(\boldsymbol{x})=P(\boldsymbol{x})=\frac{1}{N} \sum_{i=1}^{N} p_{i} \quad \text { subject to } \quad T(\boldsymbol{x}) \leq T_{\max }
$$

where $f(\boldsymbol{x})$ is referred as the objective function that is to be optimized (minimized), $i$ denotes a node at the entrance of the metering section of the single-screw extruder, $N$ is the total number of nodes at the entrance of the metering section, $p_{i}$ is the pressure at an entrance node, $T(\boldsymbol{x})$ is the average temperature at the exit of the extruder, $T_{\max }$ is the maximum temperature allowed for a given polymer at the exit of the extruder and $\boldsymbol{x}$ is the vector of design parameters that are to be optimized. As discussed earlier, the design parameters for screw geometry optimization were selected to be the screw pitch and the screw depth in the metering section of a single-screw extruder.

The optimization problem formulated in Equation 4.1 is a constrained optimization problem with an inequality constraint. In general, finding an optimum point for constrained problems is more difficult compared to that for unconstrained problems. For constrained problems, it is also easier to enforce equality constraints than enforcing inequality constaints. Since it is not known a priori if the temperature constaint will be active for the optimization problem given in Equation 4.1, following optimization strategy was 
implemented in this work:

$\triangleright$ Iterate to find the minimum of $P(\boldsymbol{x})$ without enforcing any temperature constraint

$\triangleright$ If the temperature at the exit of the extruder exceeds the allowable temperature $\left(T_{\max }\right)$ at any iteration in optimization algorithm, enforce the temperature constraint as an equality contraint $\left(T(\boldsymbol{x})=T_{\max }\right)$

It has been established in the literature that the optimization strategy discussed above can be implemented by using the Augmented Lagrangian Method with an extension to inequality constraints $[95,96]$. The details about the Augmented Lagrangian Method is given in the next section.

\subsection{Augmented Lagrangian Method: Extension to Inequality Constraints}

The Augmented Lagrangian Method combines the properties of the Lagrangian Method and Quadratic Penalty Method [97]. For constrained problems with equalities, the objective function $f(\boldsymbol{x})$ in Equation 4.1 is redefined as:

$$
\mathcal{L}_{\mathcal{A}}(\boldsymbol{x}, \lambda ; \mu)=f(\boldsymbol{x})-\sum_{e} \lambda_{e} c_{e}(\boldsymbol{x})+\frac{\mu}{2} \sum_{e} c_{e}(\boldsymbol{x})^{2}
$$

where $\boldsymbol{x}$ is the vector of design parameters that are to be optimized, $\lambda$ is the Lagrange multipliers vector, $\mu$ is the penalty parameter, $c(\boldsymbol{x})$ is the constraint function, and $e$ is the total number of equality constraints. The modified objective function (Equation 4.2) is referred as Augmented Lagrangian function. For the methods based on the Augmented Lagrangian function, the minimization of this function gives the solution of the optimization problem with the Lagrange multipliers fixed at their optimum values. 
However, the values of the Lagrange multipliers is not known a priori. Thus, an iterative scheme is needed to find a solution to the optimization problem. In this iterative scheme, first, the value of the Lagrange multipliers and the penalty parameter are fixed and the Augmented Lagrangian function is minimized with respect to the design variables. Next, the values of the Lagrange multipliers and the penalty parameter are updated. In order to satisfy the optimality conditions [97], the updated Lagrange multipliers are in the following form:

$$
\lambda_{e}^{(k+1)}=\lambda_{e}^{(k)}-\mu^{(k)} c_{e}\left(\boldsymbol{x}^{(k)}\right)
$$

where $k$ denotes the iteration number. This iterative process is continued until the convergence is achieved for the Lagrange multipliers or the design variables. In theory, if the convergence is achieved for either Lagrange multipliers or the design variables, the convergence criteria for the other term is also satisfied.

\subsubsection{Extension to Inequality Constraint}

An optimization problem with inequality constraints can be converted into a problem with equality constraints by defining slack variables $\left(s_{i e}\right)[95,96]$. This conversion can be formulated as follows:

$$
\min _{\boldsymbol{x}} f(\boldsymbol{x}) \quad \text { subject to } \quad c_{i e}(x) \geq 0
$$

converted to

$$
\min _{\boldsymbol{x}, \boldsymbol{s}} f(\boldsymbol{x}) \quad \text { subject to } \quad c_{i e}(x)-s_{i e}=0 \quad s_{i e} \geq 0
$$


where subscript ie denotes the number of inequality constraints. By implementing the Augmented Lagrangian Method (Equation 4.2) to the optimization problem in Equation 4.4 , the subproblem that needs to be solved at the $k^{\text {th }}$ iteration of the scheme discussed in Section 4.2 is obtained as:

$$
\begin{array}{r}
\min _{\boldsymbol{x}, \boldsymbol{s}} \mathcal{L}_{\mathcal{A}}=f(\boldsymbol{x})-\sum_{i e} \lambda_{i e}^{(k)}\left(c_{i e}\left(\boldsymbol{x}^{(\boldsymbol{k})}\right)-s_{i e}\right)+\frac{\mu^{(k)}}{2} \sum_{i e}\left(c_{i e}\left(\boldsymbol{x}^{(\boldsymbol{k})}\right)-s_{i e}\right)^{2} \\
\text { subject to } \quad s_{i e} \geq 0
\end{array}
$$

As given in Equation 4.5, the function to be minimized is a convex quadratic function with respect to $s_{i e}$ since the second derivative of the function with respect to $s_{i e}$ is positive. Therefore, an explicit unconstrained minimization of the equation shown above with respect to $s_{i e}$ can be found when the partial derivative of this equation with respect to $s_{i e}$ is equal to zero. This unconstrained minimizer is given in Equation 4.6.

$$
\lambda_{i e}^{(k)}-\mu^{(k)}\left(c_{i e}\left(\boldsymbol{x}^{(\boldsymbol{k})}\right)-s_{i e}\right)=0 \rightarrow s_{i e}=c_{i e}\left(\boldsymbol{x}^{(\boldsymbol{k})}\right)-\frac{\lambda_{i e}^{(k)}}{\mu^{(k)}}
$$

If the value of $s_{i e}$ is smaller than zero, then the optimal value of $s_{i e}$ in Equation 4.5 is zero, since the objective function is convex in $s_{i e}$ as discussed earlier and the objective function is subjected to a value of $s_{i e}$ greater and equal to zero. Thus, the optimal value of $s_{i e}$ can be given as:

$$
s_{i e}=\max \left(c_{i e}\left(\boldsymbol{x}^{(\boldsymbol{k})}\right)-\frac{\lambda_{i e}^{(k)}}{\mu^{(k)}}, 0\right)
$$

By substituting the slack variables (Equation 4.7) in Equation 4.5, the equivalent form of the minimization problem can be written as:

$$
\min _{\boldsymbol{x}} \mathcal{L}_{\mathcal{A}}
$$


where

$$
\mathcal{L}_{\mathcal{A}}= \begin{cases}f(\boldsymbol{x})-\frac{1}{2 \mu^{(k)}} \sum_{i e}\left(\lambda_{i e}^{(k)}\right)^{2} & \text { if } c_{i e}\left(\boldsymbol{x}^{(\boldsymbol{k})}\right)-\frac{\lambda_{i e}^{(k)}}{\mu^{(k)}} \geq 0 \\ f(\boldsymbol{x})-\sum_{i e} \lambda_{i e}^{(k)} c_{i e}\left(\boldsymbol{x}^{(\boldsymbol{k})}\right)+\frac{\mu^{(k)}}{2} \sum_{i e}\left(c_{i e}\left(\boldsymbol{x}^{(\boldsymbol{k})}\right)\right)^{2} & \text { else }\end{cases}
$$

This natural extension of the Augmented Lagrangian method to the inequality-constrained case can be used in the iterative scheme that is discussed in Section 4.2 with the modification of Equation 4.3. Once the approximate solution $\boldsymbol{x}^{(\boldsymbol{k})}$ is obtained at iteration $k$, the Lagrange multipliers should be updated using the following formula:

$$
\lambda_{i e}^{(k+1)}=\max \left(\lambda_{i e}^{(k)}-\mu^{(k)} c_{i e}\left(\boldsymbol{x}^{(\boldsymbol{k})}\right), 0\right)
$$

This modification in the update of the Lagrange multiplier (Equation 4.9) is due to the fact that the derivative of the Augmented Lagrangian function with respect to design variables should be close to zero at an approximate solution $\boldsymbol{x}^{(k)}$.

\subsection{Optimization Algorithm}

The Augmented Lagrangian Method discussed in Section 4.2.1 was used in the code that was develop to optimize the problem formulated in Equation 4.1. After implementing the Augmented Lagrangian Method to the optimization problem (Equation 4.1), the Augmented Lagrangian function $\mathcal{L}_{\mathcal{A}}$ that needs to be minimized at the $k^{\text {th }}$ iteration of the optimization scheme can be written as:

$$
\mathcal{L}_{\mathcal{A}}= \begin{cases}P\left(\boldsymbol{x}^{(\boldsymbol{k})}\right)-\frac{1}{2 \mu^{(k)}}\left(\lambda^{(k)}\right)^{2} & \text { if } c\left(\boldsymbol{x}^{(\boldsymbol{k})}\right)-\frac{\lambda^{(k)}}{\mu^{(k)}} \geq 0 \\ P\left(\boldsymbol{x}^{(\boldsymbol{k})}\right)-\lambda^{(k)} c\left(\boldsymbol{x}^{(\boldsymbol{k})}\right)+\frac{\mu^{(k)}}{2}\left(c\left(\boldsymbol{x}^{(\boldsymbol{k})}\right)\right)^{2} & \text { else }\end{cases}
$$


where

$$
c\left(\boldsymbol{x}^{(\boldsymbol{k})}\right)=\frac{T_{\max }}{T\left(\boldsymbol{x}^{(\boldsymbol{k})}\right)}-1
$$

The algorithm implemented in the optimization code is described in Algorithm 4.1. As shown in line 6 of Algorithm 4.1, in order to update the Lagrange multipliers and to test for convergence at the $k^{\text {th }}$ iteration, the value of the constraint function has to be greater than $-\eta^{(k)}$, that is, the temperature at the exit of the extruder should be sufficiently smaller than the maximum temperature allowed at the exit of the extruder. If this condition holds, then the optimization problem reduces to an unconstrained problem, the value of the penalty parameter is kept the same and Lagrange multipliers are updated for the next iteration. However, if the condition of sufficiently small exit temperature does not hold, then the penalty parameter is increased to ensure that for the next subproblem minimization, the violation on the constraint is decreased.

The constants A, B, C, D, and E given in Algorithm 4.1 are arbitrary numbers [97] and may vary for different problems. In this research, values of $4.0,2.0,0.9,1.5$, and 2.0 were used for the parameters of $A, B, C, D$, and $E$, respectively. For the values of convergence tolerances $\eta^{*}$ and $\omega^{*}$, a value of 0.01 was used.

In order to solve the subproblem shown in the line 5 of the Algorithm 4.1, a line search method [97] was applied in $k^{t h}$ iteration of the optimization algorithm. The minimization of the Augmented Lagrangian function $\mathcal{L}_{\mathcal{A}}$ was achieved through updating the design variables $\boldsymbol{x}^{(k)}$ in a manner such that:

$$
\boldsymbol{x}_{\boldsymbol{i}+\mathbf{1}}^{(k)}=\boldsymbol{x}_{\boldsymbol{i}}^{(\boldsymbol{k})}+\alpha_{i}^{(k)} \boldsymbol{p}_{\boldsymbol{i}}
$$

where $i$ denotes the number of iterations in the line search method algorithm, $k$ is the number of iterations in the Augmented Lagrangian method algorithm, $\alpha$ is the step length 


\section{Algorithm 4.1 Optimization code algorithm}

1: Choose initial values for the design parameters $\boldsymbol{x}^{(0)}$ and Lagrange multipliers $\lambda^{(0)}$.

For Lagrange multipliers it is selected to be zero

2: Choose convergence tolerances $\eta^{*}$ and $\omega^{*}$

3: Set $\mu^{(0)}=A, \omega^{(0)}=1 / \mu^{(0)}, \eta^{(0)}=1 /\left(\mu^{(0)}\right)^{B}$

4: for $k=0$, maximum iteration do

5: Find an approximate solution $\boldsymbol{x}^{(k)}$ of the subproblem such that:

$$
\left\|\nabla \mathcal{L}_{\mathcal{A}}\left(\boldsymbol{x}^{(k)}, \boldsymbol{\lambda}^{(k)} ; \mu\right)\right\| \leq \omega^{(k)}
$$

6: $\quad$ if $c\left(\boldsymbol{x}^{(\boldsymbol{k})}\right) \geq-\eta^{(k)}$ then

7: $\quad$ (Test for convergence)

8: $\quad$ if $c\left(\boldsymbol{x}^{(k)}\right) \geq-\eta^{*}$ AND $\left\|\nabla \mathcal{L}_{\mathcal{A}}\left(\boldsymbol{x}^{(k)}, \boldsymbol{\lambda}^{(k)} ; \mu\right)\right\| \leq \omega^{*}$ then

9: $\quad$ Return the approximate solution $\boldsymbol{x}^{(k)}$

10: end if

11: (Update the Lagrange multipliers and tighten tolerances)

12: $\quad \lambda^{(k+1)}=\max \left(\lambda^{(k)}-\mu^{(k)} c\left(\boldsymbol{x}^{(k)}\right), 0\right)$

13: $\quad \mu^{(k+1)}=\mu^{(k)}$

14: $\quad \eta^{(k+1)}=\eta^{(k)} /\left(\mu^{(k+1)}\right)^{C}$

15: $\quad \omega^{(k+1)}=\omega^{(k)} / \mu^{(k+1)}$

16: else $\{$ Increase the penalty parameter and tighten tolerances\}

17: $\quad \lambda^{(k+1)}=\lambda^{(k)}$

18: $\quad \mu^{(k+1)}=D \mu^{(k)}$

19: $\quad \eta^{(k+1)}=1 /\left(\mu^{(k+1)}\right)^{E}$

20: $\quad \omega^{(k+1)}=1 / \mu^{(k+1)}$

21: end if

22: Choose a new starting point, i.e. $x^{(k+1)}=x^{(k)}$

23: end for 
which satisfies the Wolfe conditions (sufficient decrease and curvature conditions [97]), and $\boldsymbol{p}$ is the descent (search) direction vector for the Augmented Lagrangian function $\mathcal{L}_{\mathcal{A}}$ at $\boldsymbol{x}_{i}^{(k)}$. The Wolfe conditions, namely, sufficient decrease and curvature conditions, ensure a reasonable progress in the line search algorithm. Satisfying the sufficient decrease condition avoids insufficient decrease in the Augmented Lagrangian function value at each iteration of the line search method whereas satisfying the curvature condition eliminates the unacceptably short step lengths. In this work, only the sufficient decrease condition, given in Equation 4.12, with a backtracking algorithm [97] was enforced since the curvature condition requires the computation of $\nabla \mathcal{L}_{\mathcal{A}}$ twice in each iteration of the line search method and each computation of Augmented Lagrangian function gradient was computationally expensive. The backtracking algorithm was employed to find an appropriate value for the step length $\alpha^{(k)}$.

$$
\mathcal{L}_{\mathcal{A}}\left(\boldsymbol{x}_{\boldsymbol{i}}^{(\boldsymbol{k})}+\alpha_{i}^{(k)} \boldsymbol{p}_{\boldsymbol{i}}\right) \leq \mathcal{L}_{\mathcal{A}}\left(\boldsymbol{x}_{\boldsymbol{i}}^{(\boldsymbol{k})}\right)+c_{1} \alpha^{(k)}\left(\nabla \mathcal{L}_{\mathcal{A}}\left(\boldsymbol{x}_{\boldsymbol{i}}^{(\boldsymbol{k})}\right)\right)^{T} \boldsymbol{p}_{\boldsymbol{i}}
$$

$c_{1}$ given in Equation 4.12 is a constant number such that $c_{1} \in(0,1) . c_{1}$ was set to 0.001 in this work.

A fundamental method described in the literature to determine the descent direction $\boldsymbol{p}_{\boldsymbol{i}}$ in Equation 4.11 is the Newton's method. The descent direction in Newton's method is defined as:

$$
\boldsymbol{p}_{\boldsymbol{i}}=-\left(\nabla^{2} \mathcal{L}_{\mathcal{A}}\left(\boldsymbol{x}_{\boldsymbol{i}}^{(\boldsymbol{k})}\right)\right)^{-1} \nabla \mathcal{L}_{\mathcal{A}}\left(\boldsymbol{x}_{\boldsymbol{i}}^{(\boldsymbol{k})}\right)
$$

As shown in Equation 4.13, the computation of the descent direction in Newton's method requires the first and second derivatives of the Augmented Lagrangian function. Even though the descent direction given in Equation 4.13 results in a faster local convergence in the line search method, the computation of the second derivative of the 
objective function is a computationally expensive task and erroneous results may occur if the finite difference method is used for the gradient computations. Thus, instead of using the Newton's method, a Quasi-newton method was used in this work to find the descent direction. In a Quasi-newton method, the computation of the second derivatives are avoided by approximating the Hessian $\nabla^{2} \mathcal{L}_{\mathcal{A}}\left(\boldsymbol{x}_{\boldsymbol{i}}^{(\boldsymbol{k})}\right)$ or inverse Hessian $\left(\nabla^{2} \mathcal{L}_{\mathcal{A}}\left(\boldsymbol{x}_{\boldsymbol{i}}^{(\boldsymbol{k})}\right)\right)^{-1}$ matrix. In each iteration of a line search algorithm, a Quasi-Newton method provides an update of the approximate Hessian (or inverse Hessian) matrix. In this work, a Quasi-Newton method, named BFGS method [97], was used to update the approximate inverse Hessian matrix $\left(H_{i}\right)$. The descent direction $\left(\boldsymbol{p}_{\boldsymbol{i}}\right)$ and the Hessian update $\left(H_{i+1}\right)$ in BFGS method is defined as [97]:

$$
\begin{gathered}
\boldsymbol{p}_{\boldsymbol{i}}=-H_{i} \nabla \mathcal{L}_{\mathcal{A}}\left(\boldsymbol{x}_{\boldsymbol{i}}^{(\boldsymbol{k})}\right) \\
H_{i+1}=\left(I-\rho_{i} \boldsymbol{s}_{\boldsymbol{i}} \boldsymbol{y}_{\boldsymbol{i}}^{\boldsymbol{T}}\right) H_{i}\left(I-\rho_{i} \boldsymbol{y}_{\boldsymbol{i}} \boldsymbol{s}_{\boldsymbol{i}}^{\boldsymbol{T}}\right)+\rho_{i} \boldsymbol{s}_{\boldsymbol{i}} \boldsymbol{s}_{\boldsymbol{i}}^{\boldsymbol{T}}
\end{gathered}
$$

where

$$
\begin{aligned}
\rho_{i} & =\frac{1}{\boldsymbol{y}_{i}^{\boldsymbol{T}} \boldsymbol{s}_{\boldsymbol{i}}} \\
\boldsymbol{y}_{\boldsymbol{i}} & =\nabla \mathcal{L}_{\mathcal{A}}\left(\boldsymbol{x}_{i+1}^{(k)}\right)-\nabla \mathcal{L}_{\mathcal{A}}\left(\boldsymbol{x}_{i}^{(k)}\right) \\
\boldsymbol{s}_{\boldsymbol{i}} & =\boldsymbol{x}_{\boldsymbol{i}+\mathbf{1}}^{(\boldsymbol{k})}-\boldsymbol{x}_{\boldsymbol{i}}^{(\boldsymbol{k})}
\end{aligned}
$$

The BFGS algorithm used to solve the minimization problem in line 5 of the Augmented Lagrangian Method algorithm (Algorithm 4.1) is given in Algorithm 4.2. 


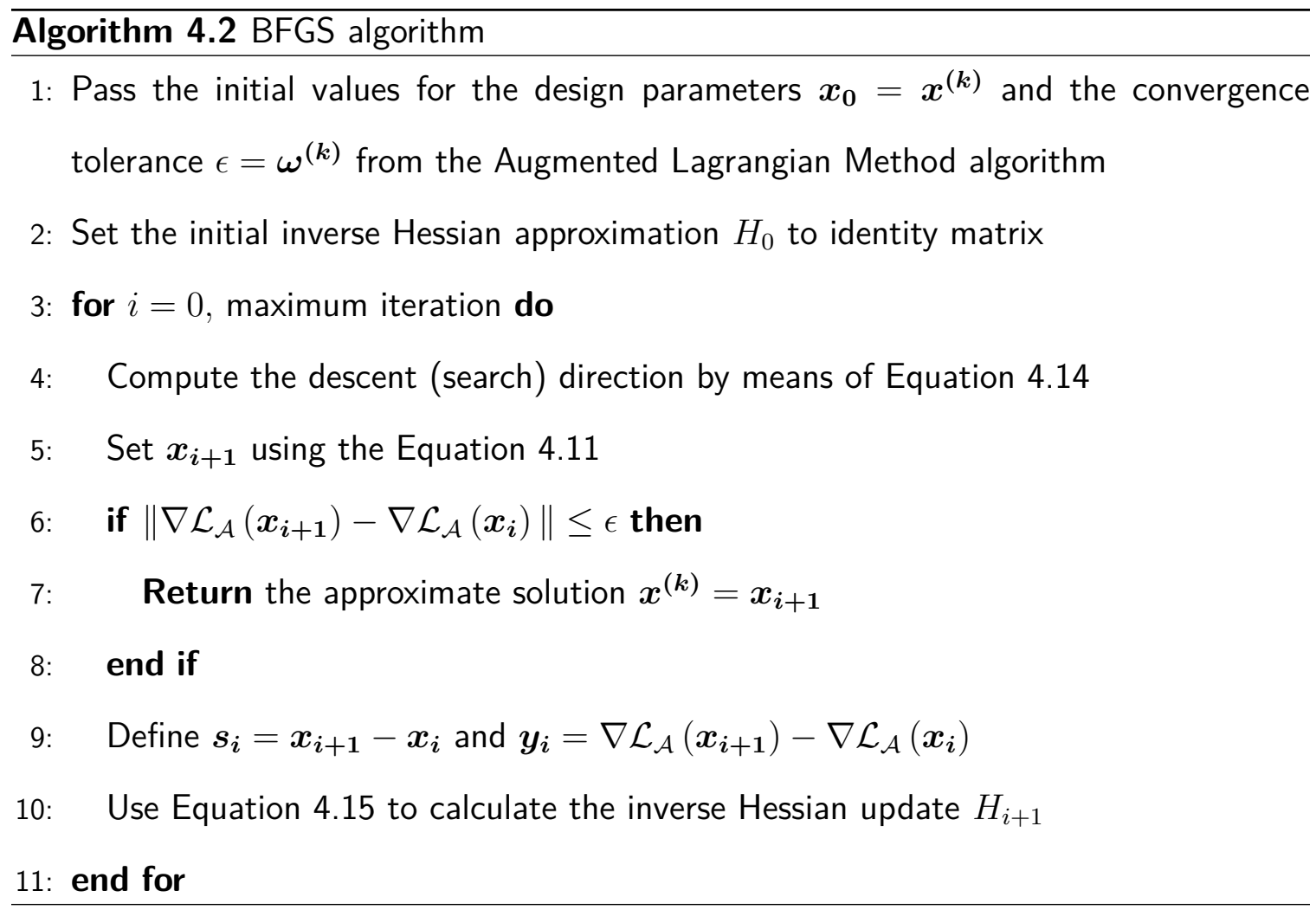

As shown in Algorithm 4.2, the derivative of the Augmented Lagrangian function $\mathcal{L}_{\mathcal{A}}$ with respect to design parameters $\boldsymbol{x}$ is needed. Since $\mathcal{L}_{\mathcal{A}}$ may be a function of velocity $(\boldsymbol{u})$, pressure $(p)$ and temperature $(T)$, using the chain rule, the derivative of $\mathcal{L}_{\mathcal{A}}$ with respect to $\boldsymbol{x}$ can be written as:

$$
\frac{D \mathcal{L}_{\mathcal{A}}}{D \boldsymbol{x}_{\boldsymbol{j}}}=\frac{\partial \mathcal{L}_{\mathcal{A}}}{\partial \boldsymbol{u}} \frac{D \boldsymbol{u}}{D \boldsymbol{x}_{\boldsymbol{j}}}+\frac{\partial \mathcal{L}_{\mathcal{A}}}{\partial p} \frac{D p}{D \boldsymbol{x}_{\boldsymbol{j}}}+\frac{\partial \mathcal{L}_{\mathcal{A}}}{\partial T} \frac{D T}{D \boldsymbol{x}_{\boldsymbol{j}}}+\frac{\partial \mathcal{L}_{\mathcal{A}}}{\partial \boldsymbol{x}_{\boldsymbol{j}}}
$$

where $j$ is the number of design parameters which is equal to two in our work since only two parameters of a screw in a single-screw extruder are optimized. As discussed in Equation 4.10, the Augmented Lagrangian function $\mathcal{L}_{\mathcal{A}}$ has two different forms depending on the constraint. Neither of these forms was an explicit function of the velocity $(\boldsymbol{u})$ and design parameters $(\boldsymbol{x})$. Due to this reason, the first and fourth terms on the right-hand 
side of the Equation 4.16 were eliminated and the Equation 4.16 reduced to:

$$
\frac{D \mathcal{L}_{\mathcal{A}}}{D \boldsymbol{x}_{\boldsymbol{j}}}=\frac{\partial \mathcal{L}_{\mathcal{A}}}{\partial p} \frac{D p}{D \boldsymbol{x}_{\boldsymbol{j}}}+\frac{\partial \mathcal{L}_{\mathcal{A}}}{\partial T} \frac{D T}{D \boldsymbol{x}_{\boldsymbol{j}}}
$$

In both forms of $\mathcal{L}_{\mathcal{A}}$ in Equation 4.10, $\frac{\partial \mathcal{L}_{\mathcal{A}}}{\partial p}$ is equal to one and in the first form of $\mathcal{L}_{\mathcal{A}}$ in Equation 4.10, $\frac{\partial \mathcal{L}_{\mathcal{A}}}{\partial T}$ is equal to zero. Thus, for this study, the derivative of $\mathcal{L}_{\mathcal{A}}$ with respect to $\boldsymbol{x}$ can be written as:

$$
\frac{D \mathcal{L}_{\mathcal{A}}}{D \boldsymbol{x}_{\boldsymbol{j}}}= \begin{cases}\frac{D p}{D \boldsymbol{x}_{\boldsymbol{j}}} & \text { if } c\left(\boldsymbol{x}^{(k)}\right)-\frac{\lambda^{(k)}}{\mu^{(k)}} \geq 0 \\ \frac{D p}{D \boldsymbol{x}_{\boldsymbol{j}}}+\frac{\partial \mathcal{L}_{\mathcal{A}}}{\partial T_{n}} \frac{D T_{n}}{D \boldsymbol{x}_{\boldsymbol{j}}} & \text { else }\end{cases}
$$

where $T_{n}$ is the temperature of a node at the exit of the extruder channel and $\frac{\partial \mathcal{L}_{\mathcal{A}}}{\partial T_{n}}$ is defined as:

$$
\frac{\partial \mathcal{L}_{\mathcal{A}}}{\partial T_{n}}=\frac{N T_{\max }}{\left(T_{n}\right)^{2}}\left(\lambda^{(k)}-\mu^{(k)}\left(\frac{T_{\max }}{T\left(\boldsymbol{x}^{(k)}\right)}-1\right)\right)
$$

where $N$ is the total number of nodes at the exit of the extruder channel. In order to determine the terms $\frac{D p}{D \boldsymbol{x}_{j}}$ and $\frac{D T_{n}}{D \boldsymbol{x}_{j}}$, the finite difference method was used as shown below. It should be noted here that the accuracy of the gradient calculated using finite difference method is closely related to step size $\left(x_{j}^{(2)}-x_{j}^{(1)}\right)$ and care should be taken while determining the value of the step size.

$$
\begin{aligned}
\frac{D p}{D \boldsymbol{x}_{j}} & =\frac{P\left(\boldsymbol{x}^{(2)}\right)-P\left(\boldsymbol{x}^{(\mathbf{1})}\right)}{\boldsymbol{x}_{j}^{(2)}-\boldsymbol{x}_{j}^{(1)}} \\
\frac{D T_{n}}{D \boldsymbol{x}_{j}} & =\frac{T_{n}\left(\boldsymbol{x}^{(\mathbf{2})}\right)-T_{n}\left(\boldsymbol{x}^{(\mathbf{1})}\right)}{\boldsymbol{x}_{j}^{(2)}-\boldsymbol{x}_{j}^{(1)}}
\end{aligned}
$$




\subsection{Screw Geometry Optimization}

As discussed earlier, only two screw geometry parameters are optimized in this work. These parameters are the lead (pitch) of the screw and the channel depth in the metering section of the screw. Only the metering section of the extruder was used in the optimization process and the length of the metering section was fixed at $444.5 \mathrm{~mm}$ ( 7 times the barrel diameter $(63.5 \mathrm{~mm}))$. An example of the barrel geometry used for the optimization code is shown in Fig. 4.1 (a). In all simulations, a constant temperature was specified at the entrance of the metering section such that the polymer entering the channel is completely in liquid form. A constant temperature was enforced for the barrel and screw surfaces. The flow simulations performed within the optimization code were steady, time-independent. Thus, the rotational speed of the barrel was specified whereas the screw was considered stationary. The direction of the barrel rotation was the opposite direction of the screw rotation in the experiment. The boundary conditions specified for the ABS, PC-22 and LDPE resins are given in Table 4.1. The material properties of these resins were already given in Section 2.3.1. At the exit of the extruder channel, zero-traction boundary condition was enforced and the velocity in the direction of the screw axis was specified such that the flow rate given in Table 4.1 was satisfied. The specified velocity along the circular cross-section of the extruder exit was calculated by assuming the polymer as a power-law fluid.

In Figs. 4.3 - 4.5, the contour lines shows the average pressure at the entrance and the average temperature at the exit of the barrel for the ABS, PC-22 and LDPE resins, respectively. The results from the developed optimization code discussed in previous sections are also shown in Figs. $4.3-4.5$ (symbols). In order to obtain the contour lines shown in Figs. $4.3-4.5$, first, the limits for the lead and the depth of the screw were determined. The lead of the screw is typically in the range from 0.8 to 1.3 times the screw 
Table 4.1: Boundary conditions for the ABS, PC-22 and LDPE resins specified in the numerical simulations.

Barrel temperature $(\mathrm{K})$

Screw temperature $(\mathrm{K})$

Polymer entrance temperature $(\mathrm{K})$

Flow rate $(\mathrm{kg} / \mathrm{h})$

Screw speed $(\mathrm{rpm})$

\begin{tabular}{ccc} 
& Resin & \\
\hline ABS & LDPE & PC-22 \\
\hline 523.0 & 473.0 & 523.0 \\
523.0 & 473.0 & 523.0 \\
450.0 & 423.0 & 450.0 \\
59.7 & 45.6 & 72.0 \\
60 & 60 & 60 \\
\hline
\end{tabular}

diameter. The typical limits for the depth of the screw channel in the metering section are $3 \%$ and $12 \%$ of the screw diameter. The shallow screw channels in the metering section results in a higher output temperature compared to a deeper screw channel and the deeper screw channel in general is suitable for very viscous materials. The lower and upper limits for the lead and the depth of the screw in Figs. $4.3-4.5$ was kept at $0.8-1.8$ times the screw diameter and $0.03-0.21$ times the screw diameter, respectively, to have a broader view. Using these limits for the screw geometry parameters, next, each axis corresponding to one of the screw geometry parameters was equally divided into ten intervals and a 11 by 11 grid was established. The screw geometry parameter values at each of these grid nodes were used to generate the screw geometry mesh. The screw geometry parameters other than the lead and the depth of the screw were kept the same for all screws during the screw mesh generation. This screw geometry mesh was then used to partition the barrel mesh shown in Fig. 4.1 (a) and the polymer flow in this partitioned domain was simulated with the conditions discussed above. The average pressure at the entrance and the average temperature at the exit of the partitioned barrel mesh was calculated from the simulation results for each of the grid nodes. These data obtained at each grid node 
along with the data obtained throughout the optimization code was combined and the results were plotted in Figs. $4.3-4.5$ for various resins. It should be noted here that combining the data from the optimization code, particularly from the latter optimization steps, with the data obtained at each grid node resulted in some distortion on the contour lines as clearly observed in Fig. 4.5. The contours given in Figs. $4.3-4.5$ is not a very precise representation of the average pressure at the entrance and average temperature at the exit of the partitioned barrel since limited data points were used to plot the contour lines. However, the amount of data used to plot these figures was enough to check the accuracy of the optimization code. It should also be noted here that the upper and lower limits for the screw geometry parameters were only for determining the interval in which the contour lines were generated. These limits were not enforced in the optimization code. Thus, in some cases such as in Fig. 4.5, the optimization algorithm step may be out of these limits and was not shown in the figure. In the optimization code, the only limit set was for the depth of the channel such that the depth of the channel cannot be less than zero and cannot exceed half of the screw diameter. However, the depth of the channel was found to be not close to these bounds in any optimization steps. The numbered symbols appearing in Figs. $4.3-4.5$ shows the iteration number for the Augmented Lagrangian Method algorithm. For each color, the symbol numbered with 1 shows the initial configuration of the screw geometry and the last number represents the optimized parameters for the screw geometry.

As shown in Figs. 4.3 (a) -4.5 (a), the optimization code was found to converge to a solution. Figs. $4.3(\mathrm{a})-4.5$ (a) implied that the optimization code converged to a local minimum. However, for some initial screw configurations such as blue and red symbols in Fig. 4.3 (a), and green and red symbols in Fig. 4.5 (a), it was not clear that the optimization code was converged to a local minimum. Due to this reason, the portion that shows the converged steps in the Figs. 4.3 (a) - 4.5 (a) were magnified and 


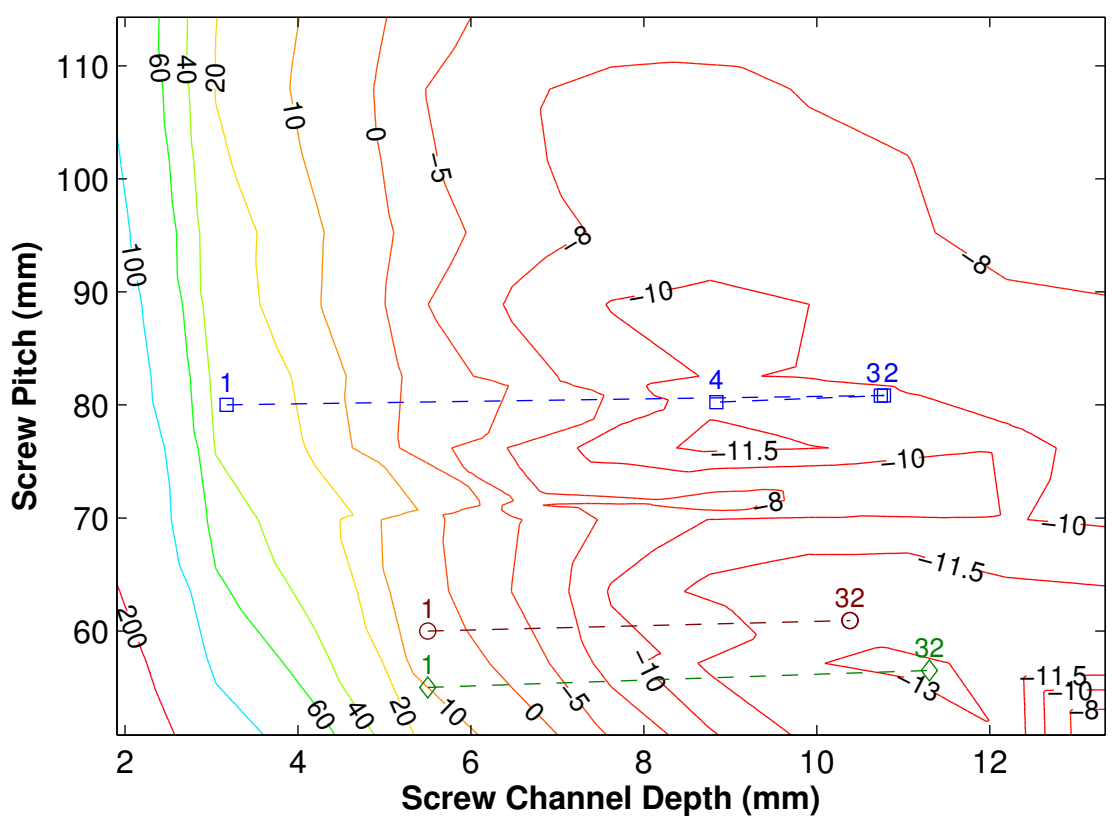

(a)

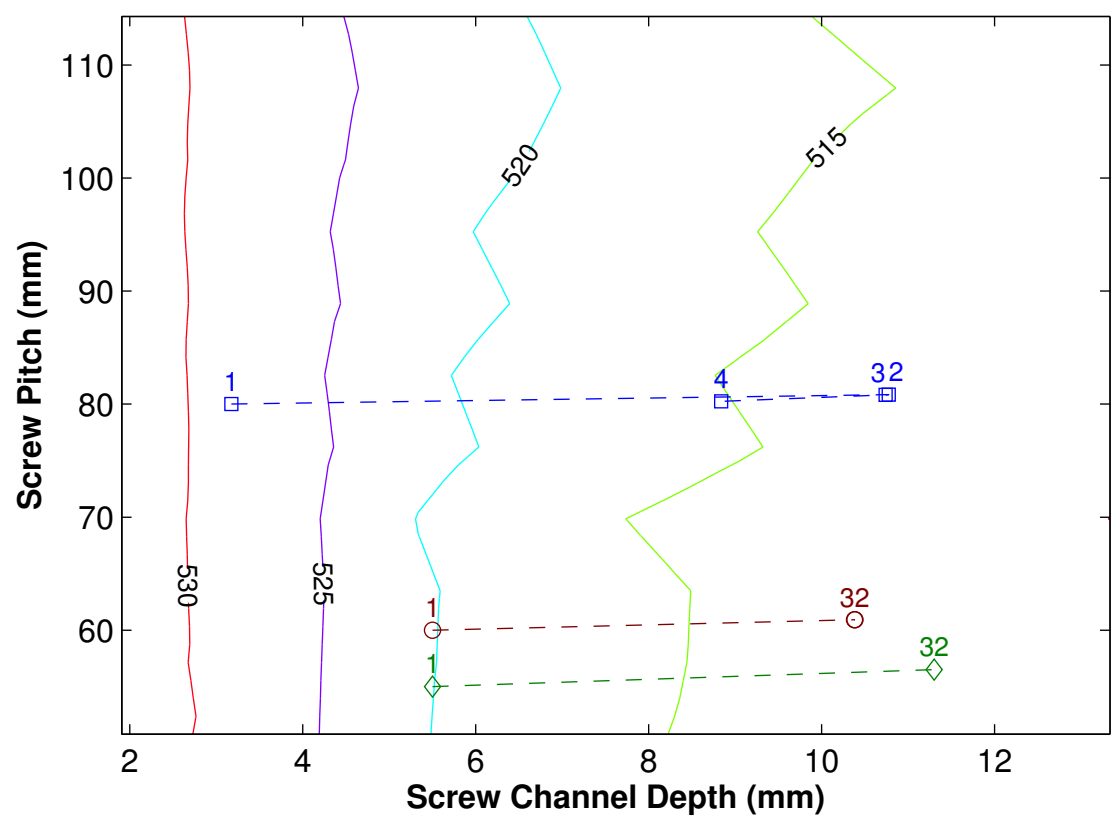

(b)

Figure 4.3: (a) Average pressure calculated at the entrance (b) average temperature calculated at the exit of the partitioned barrel mesh for the ABS resin (contour lines) and steps for the optimization algorithm (symbols with iteration numbers). 


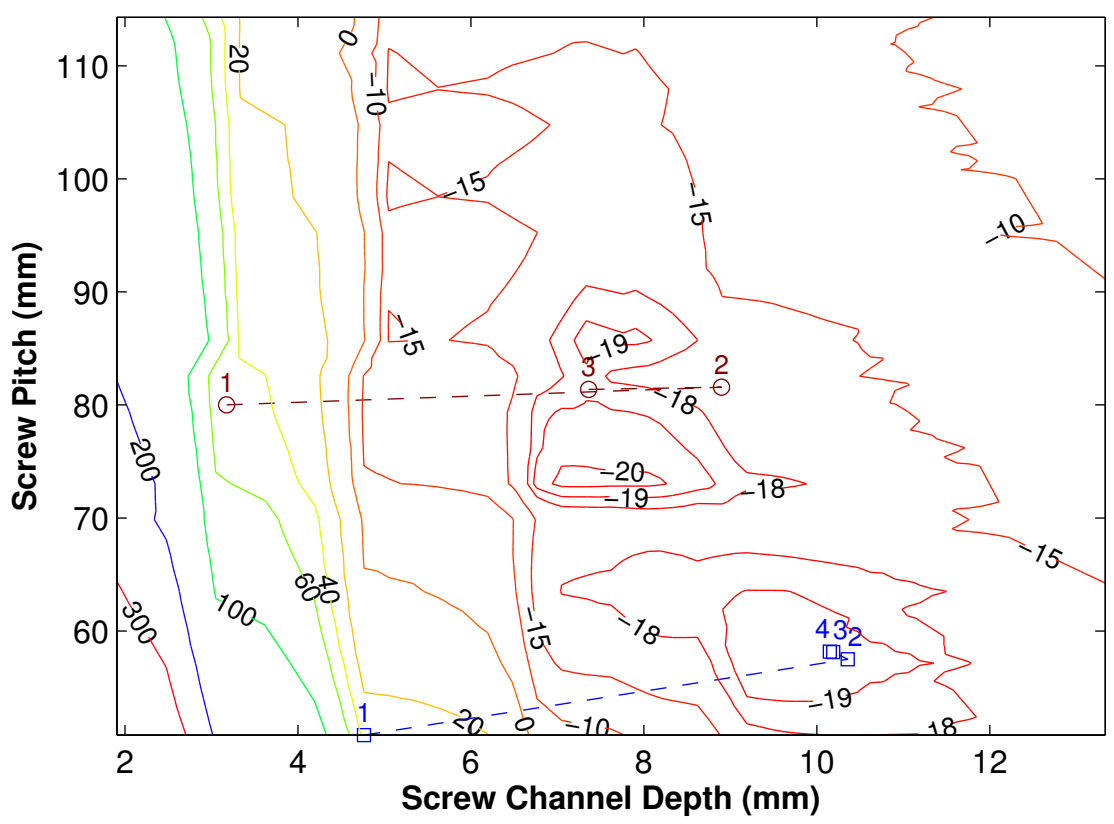

(a)

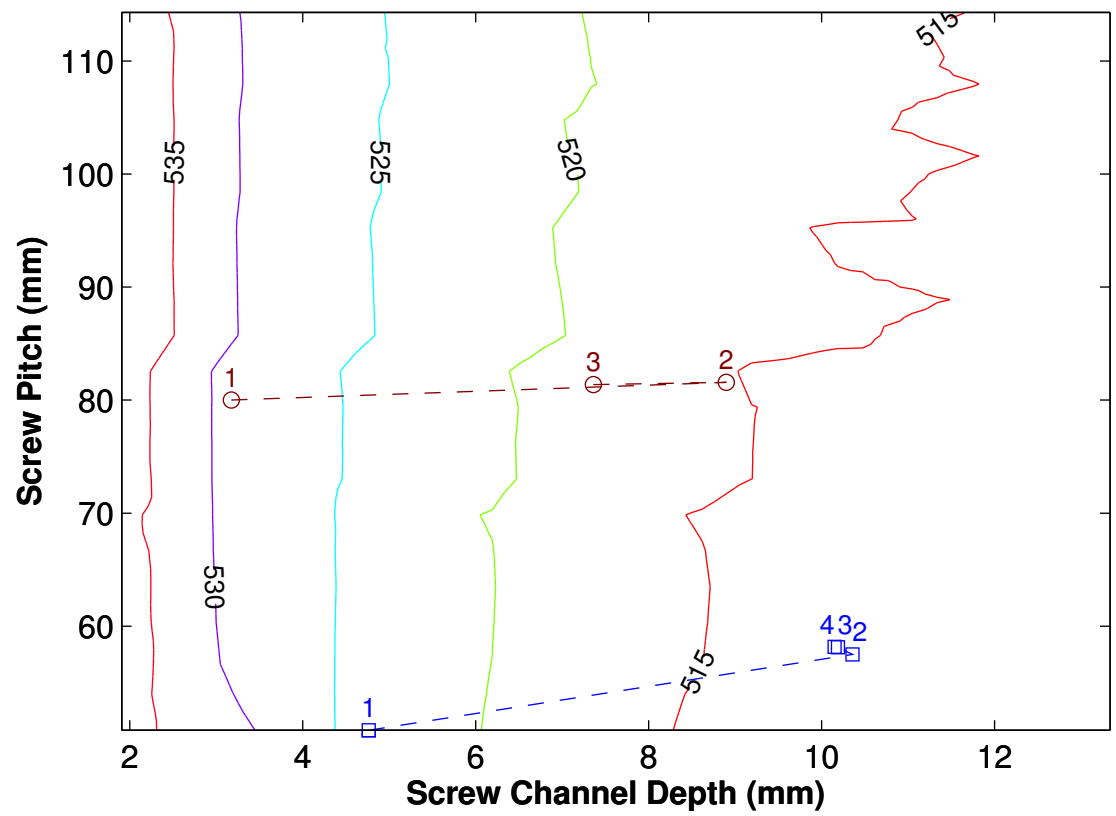

(b)

Figure 4.4: (a) Average pressure calculated at the entrance (b) average temperature calculated at the exit of the partitioned barrel mesh for the PC-22 resin (contour lines) and steps for the optimization algorithm (symbols with iteration numbers). 


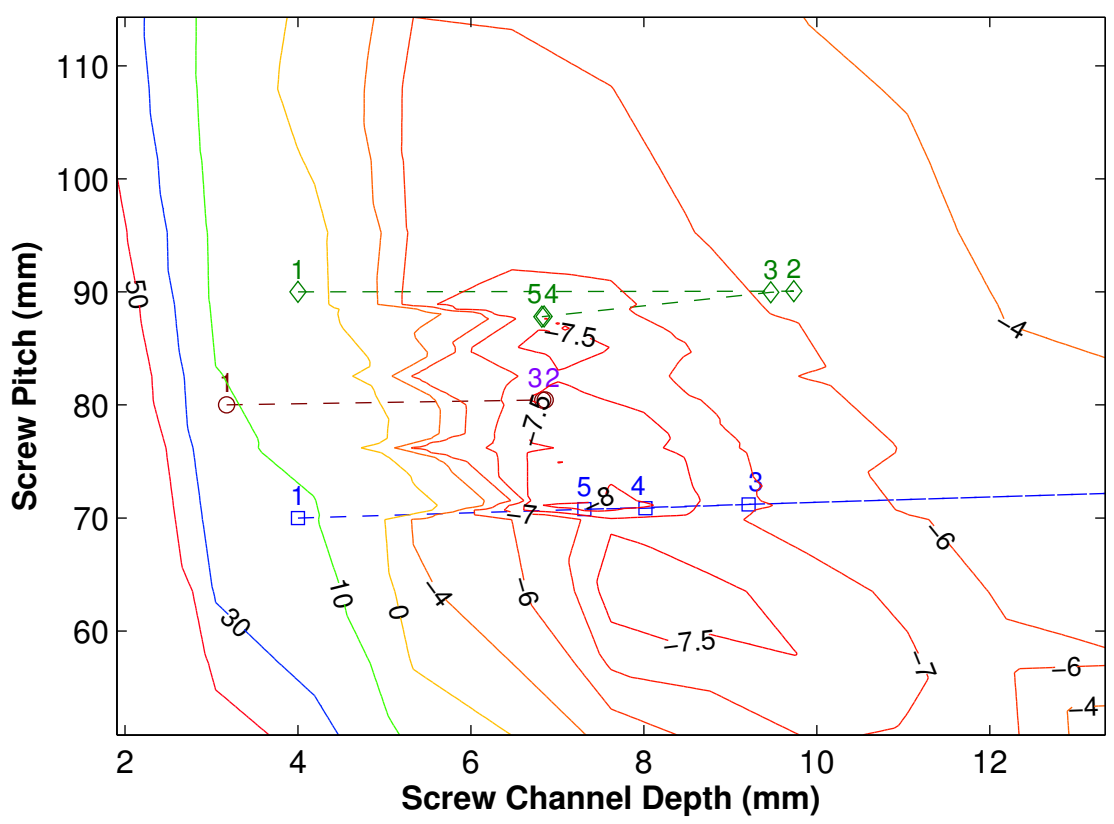

(a)

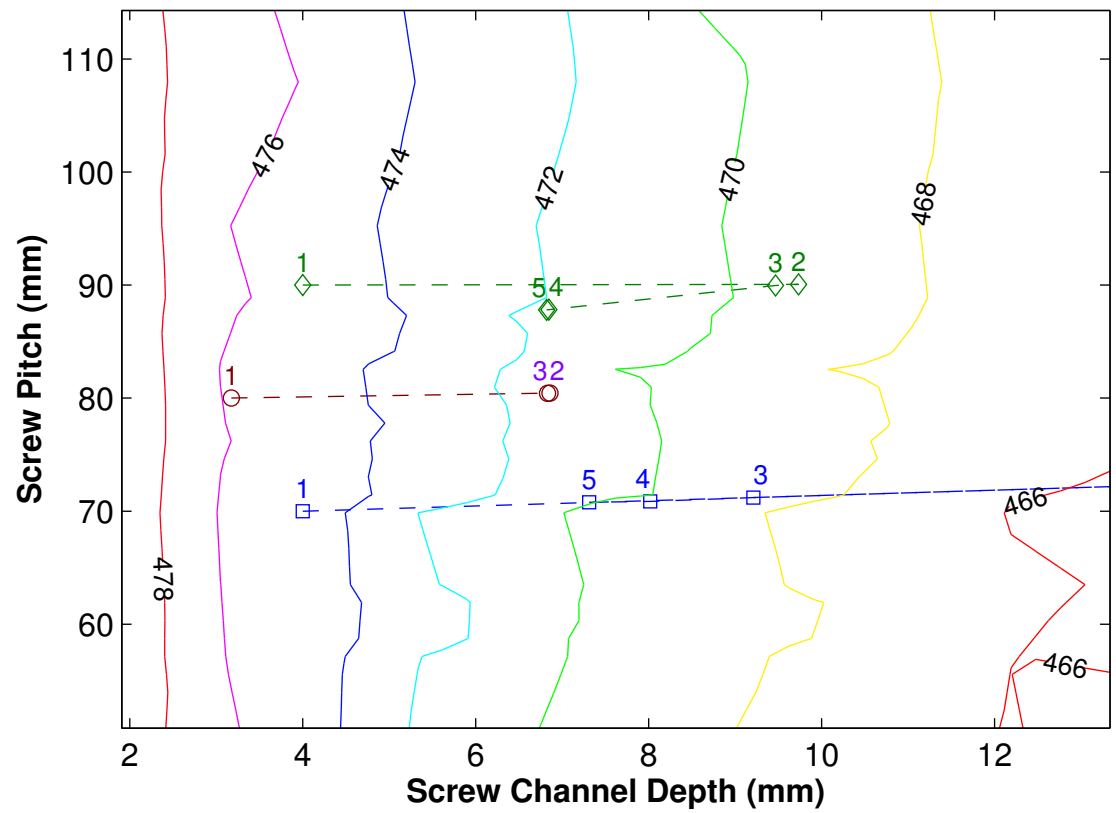

(b)

Figure 4.5: (a) Average pressure calculated at the entrance (b) average temperature calculated at the exit of the partitioned barrel mesh for the LDPE resin (contour lines) and steps for the optimization algorithm (symbols with iteration numbers). 
these magnified figures were given in Fig. 4.6. The local minimum that the optimization code converged can be clearly observed in Fig. 4.6. Even though it is desired from an optimization code to converge to a global minimum on a given search space, it is computationally expensive to search for a global minimum. Since the time to simulate the flow through the partitioned barrel geometry was in the order of several minutes and this operation was needed several times throughout the optimization code, searching for a global minimum was not attempted in this work.

The initial screw configuration with a screw lead of $80.0 \mathrm{~mm}$ and a screw depth of $3.175 \mathrm{~mm}$ in Fig. 4.4 (red symbols) was observed to converge to a saddle point. In simple terms, this converged point was a local minimum when approached from right or left in Fig. 4.4 whereas if approached from up or down direction in Fig. 4.4, this point was a local maximum. Thus, this point was both a local minimum and maximum. An easy way to recognize a saddle point in a contour plot is to look for the shape of number 8 in the contour lines. Wherever the two circles of the number 8 intersects, that point has a potential to be a saddle point. The shape of the number 8 was clearly seen in Fig. 4.4. In the current optimization algorithm dealing with the saddle point problem was avoided since it needed use of more computational resources.

For the LDPE resin, the effect of some boundary conditions such as barrel and screw temperature and flow rate on the optimized screw geometry was also investigated. The boundary conditions given in Table 4.1 for the LDPE resin was kept the same except the one whose effect was being investigated. First, the flow rate was specified at $60 \mathrm{~kg} / \mathrm{h}$ and the results obtained for the average pressure at the entrance and the average temperature at the exit of the partitioned barrel mesh along with the optimization code iteration steps are shown in Fig. 4.7. Next, similar figures were generated with a specified barrel and screw temperatures of $220{ }^{\circ} \mathrm{C}(493 \mathrm{~K})$ and the results are shown in Fig. 4.8. As shown in Fig. 4.5, 4.7 and 4.8, for the LDPE resin, in general, three local minima were observed. 


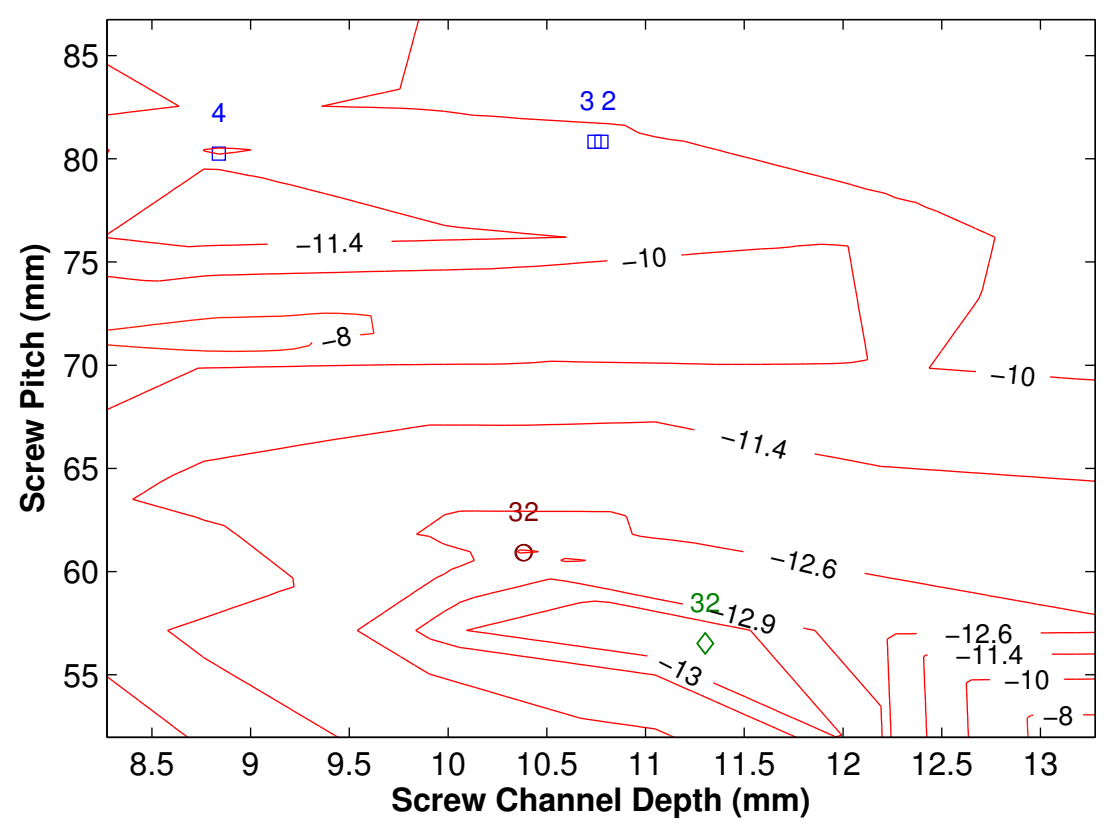

(a)

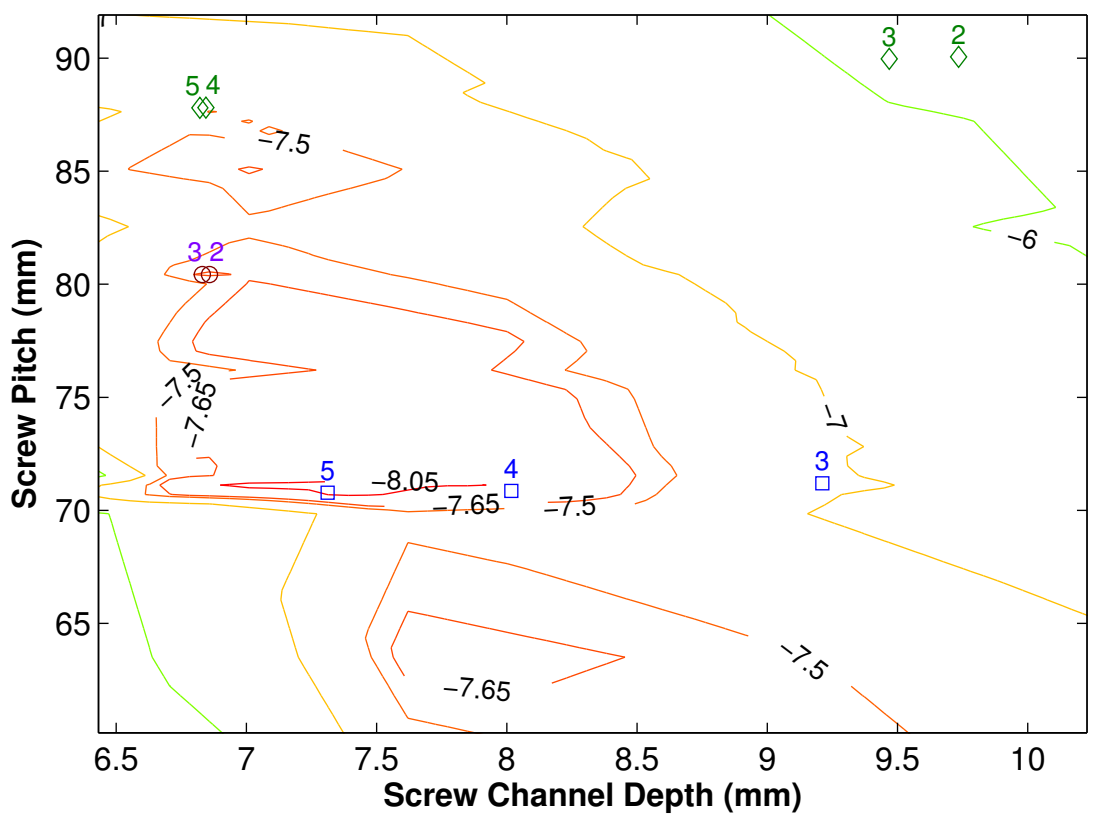

(b)

Figure 4.6: Magnified results of the Figs. 4.3 and 4.5 for the (a) ABS and (b) LDPE resins, respectively. 
With an increase in barrel and screw temperatures, no significant change was observed on the values of the screw lead and depth at these local minima. An increase in specified flow rate was also observed to have essentially no effect on the values of the screw lead at the three local minima whereas it resulted in an increase in the screw depth values at these local minima.

As shown in Figs. 4.3 (b) -4.5 (b) and Figs. 4.7 (b) -4.8 (b), at a constant screw lead length, the average exit temperature was decreasing with increasing depth of the screw in the metering section. This observation was consistent with the experience from the industry and was expected since an increase in the depth of the screw results in a decrease in heat generated through viscous dissipation causing lower temperatures in the flow channel. Figs. 4.5 (b), 4.7 (b), and 4.8 (b) show that for the same screw configuration, an increase in the specified flow rate to $60 \mathrm{~kg} / \mathrm{h}$ resulted in a slight decrease in the average temperature at the exit of the partitioned barrel whereas a $20{ }^{\circ} \mathrm{C}$ increase in the barrel and screw surface temperatures increased the average temperature at the exit of the partitioned barrel an average of $16{ }^{\circ} \mathrm{C}$. It was also observed from Figs. 4.3 (b) -4.5 (b) and Figs. 4.7 (b) -4.8 (b) that the average exit temperatures of the partitioned barrel for the optimized screw configuration are lower compared to the one calculated for the initial screw configuration. Thus, the temperature constraint in the optimization algorithm was not active in any of the optimization steps since the average temperature at the exit of the partitioned barrel was smaller than the value specified for $T_{\max }$. In order to check the optimization code for the temperature constraint at the exit, the initial values of the screw parameters for the blue symbols shown in Fig. 4.5 was used with a temperature constraint of $469.4 \mathrm{~K}$ (including the convergence tolerance) at the exit of the partitioned barrel. The results with these conditions are shown in Fig. 4.9. The blue symbols in Fig. 4.9 are the same blue symbols shown in Fig. 4.5 in which case the temperature constraint was not active. It is clear from Fig. 4.9 that the optimization code converged to the 


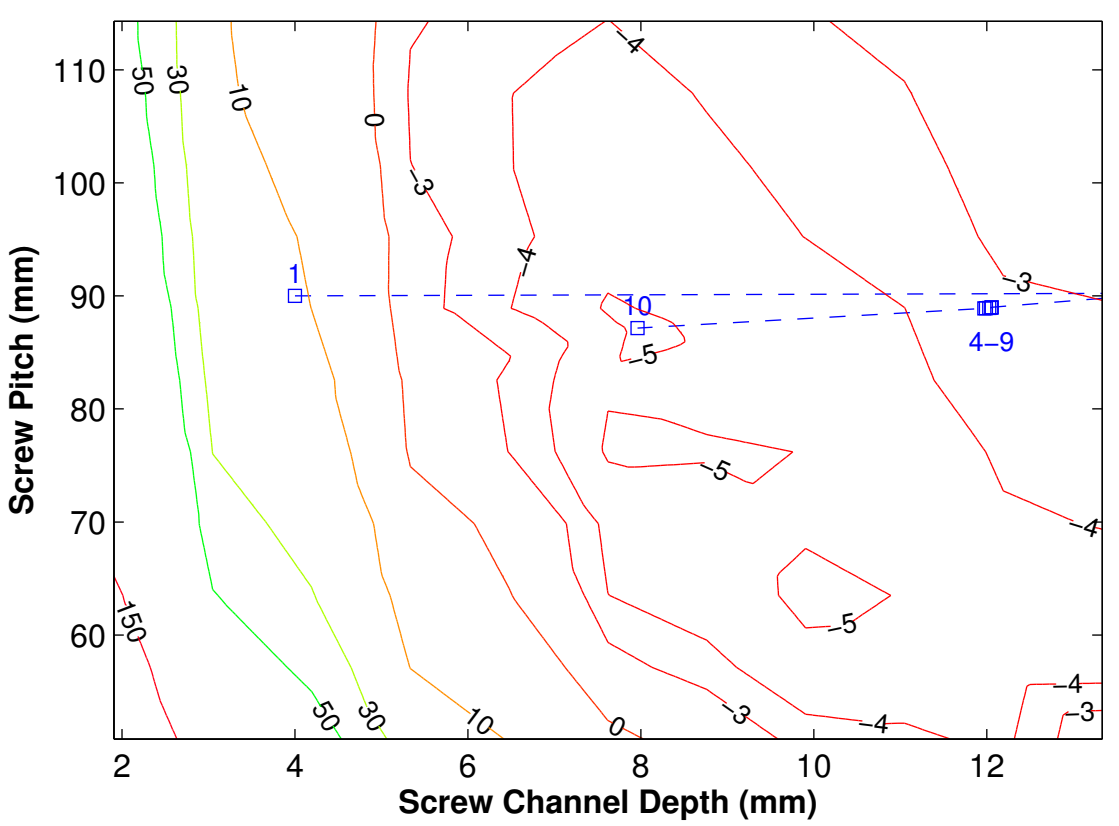

(a)

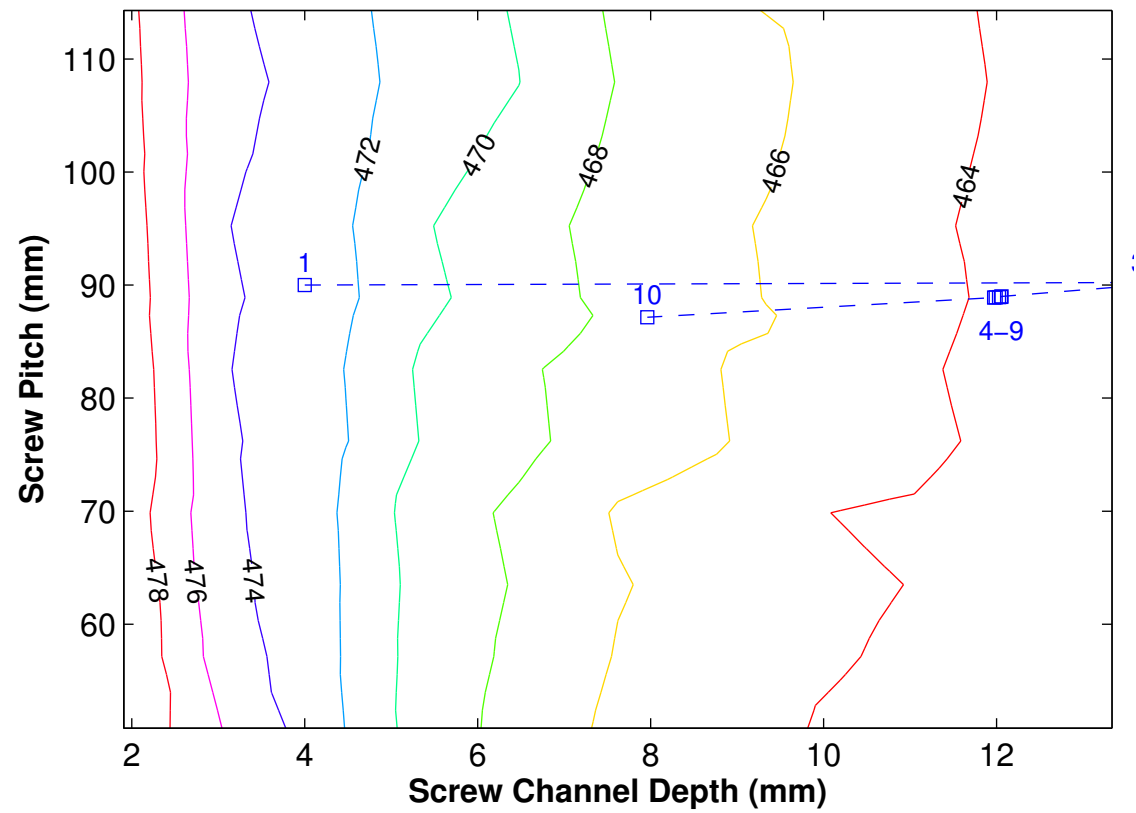

(b)

Figure 4.7: (a) Average pressure calculated at the entrance (b) average temperature calculated at the exit of the partitioned barrel mesh for the LDPE resin at a flow rate of $60 \mathrm{~kg} / \mathrm{h}$ (contour lines) and steps for the optimization algorithm (symbols). 


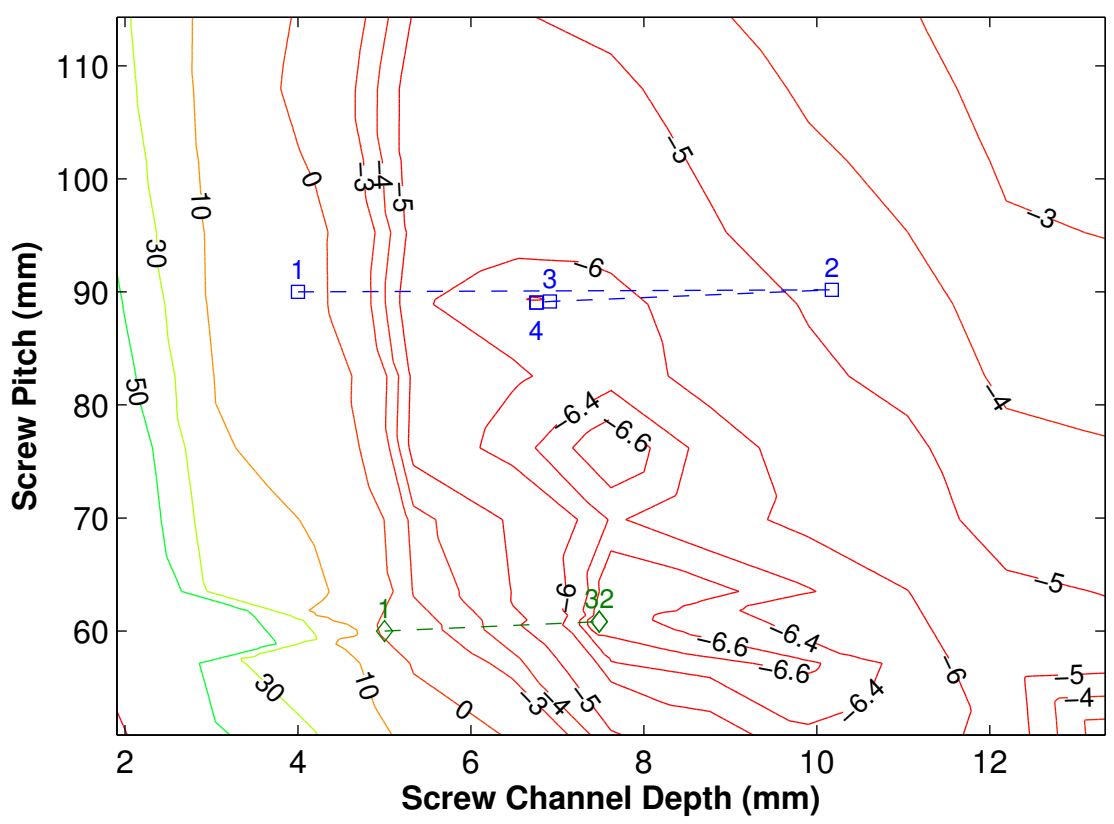

(a)

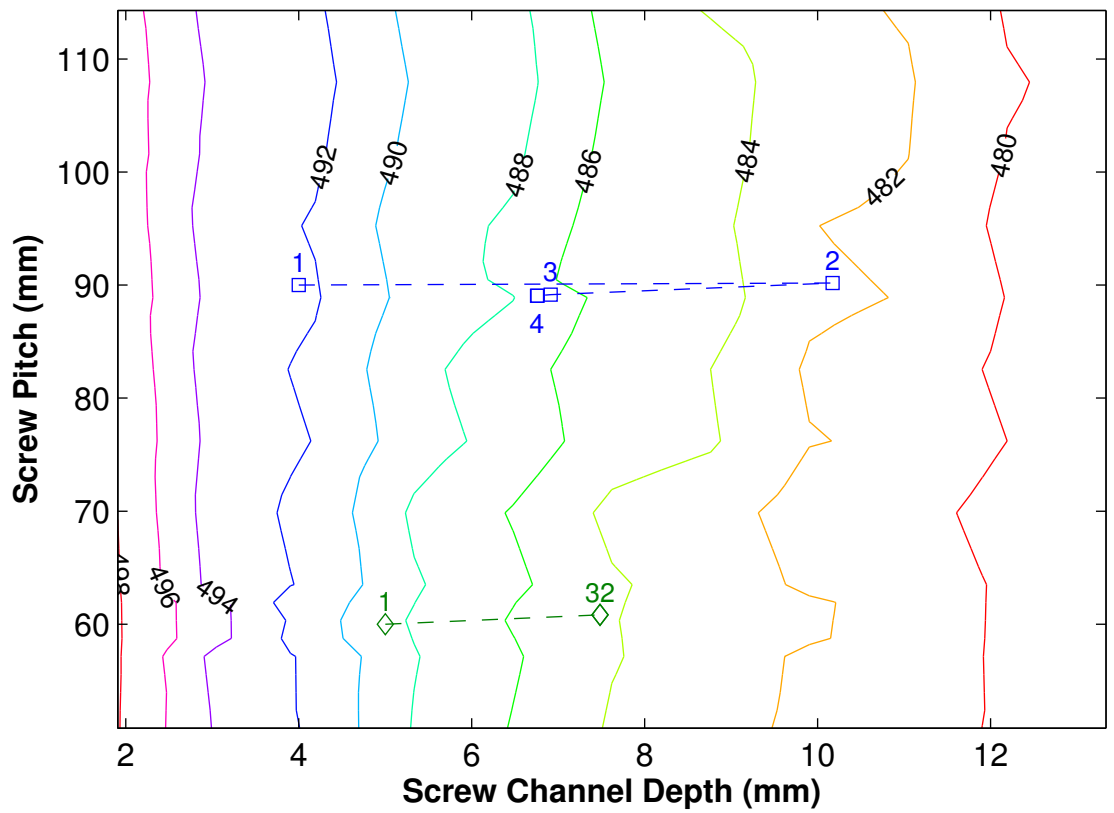

(b)

Figure 4.8: (a) Average pressure calculated at the entrance (b) average temperature calculated at the exit of the partitioned barrel mesh for the LDPE resin at a barrel/screw temperature of $220^{\circ} \mathrm{C}$ (contour lines) and steps for the optimization algorithm (symbols). 


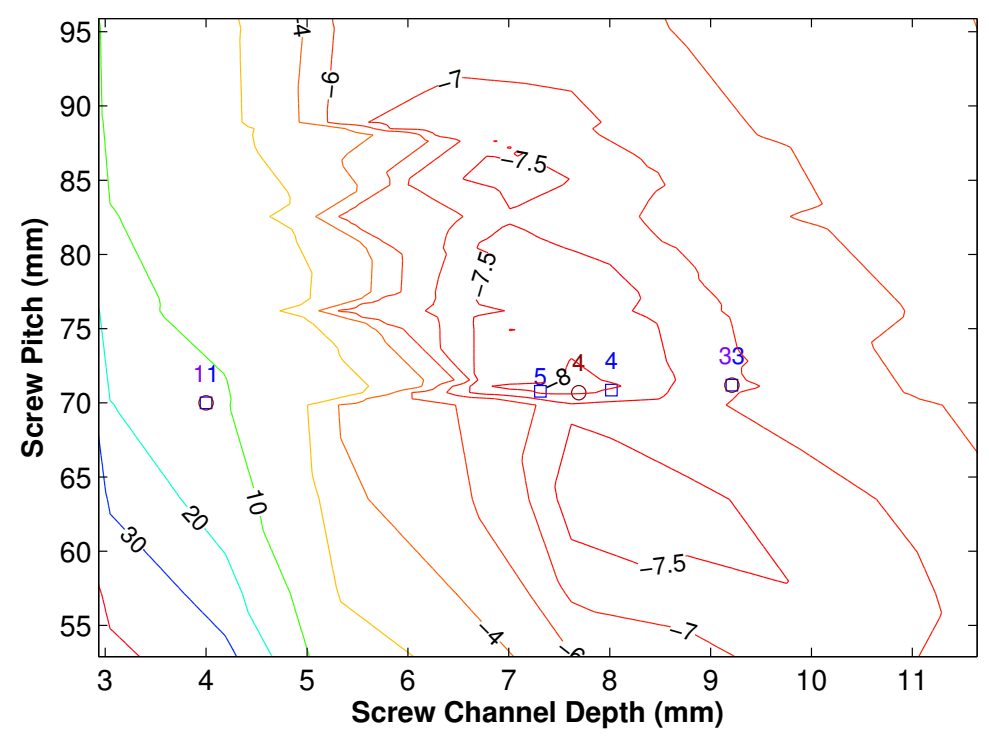

(a)

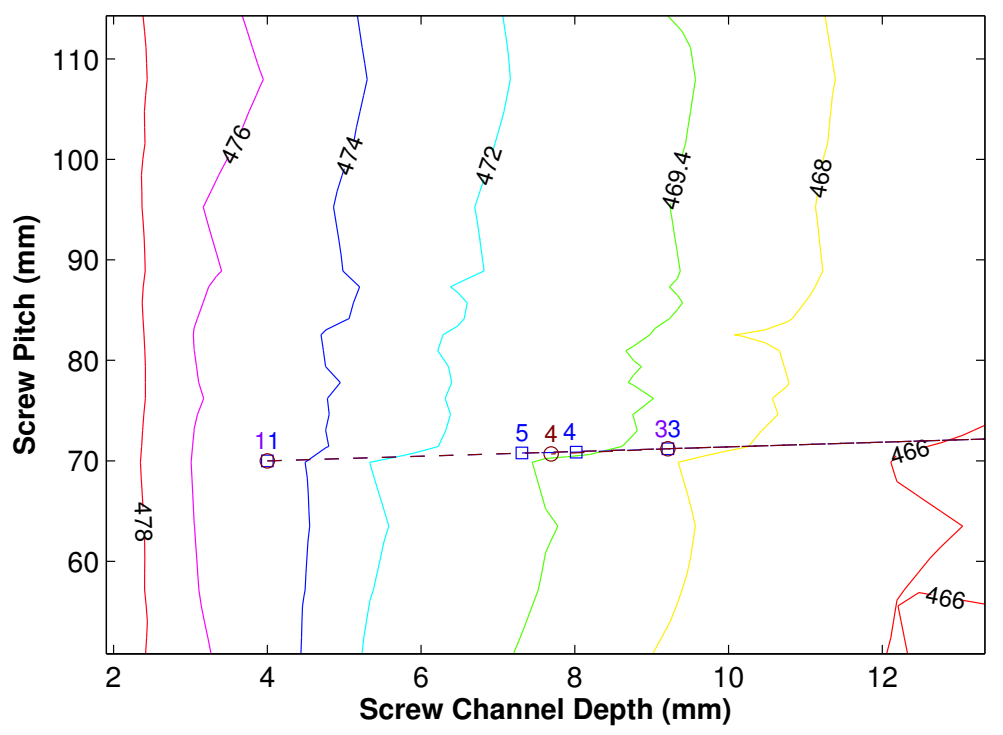

(b)

Figure 4.9: Steps of the optimization algorithm with a temperature constraint of $469.4 \mathrm{~K}$ (red symbols). The blue symbols from the optimization algorithm and the contour lines are the same as in Fig. 4.5. 
same points in the first three iterations of both cases since the temperature constraint was not violated. However, the case with a temperature constraint of $469.4 \mathrm{~K}$ at the exit of the partitioned barrel was converged to the optimized geometry in the fourth iteration without violating the temperature constraint as shown in Fig. 4.9 (b). 


\section{Chapter 5}

\section{Conclusions and Recommendations}

\section{for Future Work}

\subsection{Conclusions}

A full three-dimensional simulation of the polymer melting in single-screw extruders was performed for different screw configurations. It was observed from the experiments that for a constant axial length of compression section, an increase in compression ratio resulted in a slight increase in the melting rate and an increase in pressure rise in the compression section of the screw channel. For a fixed compression ratio, an increase in compression rate also increased the melting in the extruder but decreased the pressure rise in the channel. Even though some discrepancies existed between the numerical predictions and experimental data, both experiments and simulations exhibited the Maddock melting mechanism and had a good agreement on melting profile and pressure variation in the channel. The sensitivity of melting profile to various material properties and processing parameters was also analyzed. With a constant flow rate enforced at the entrance of the screw channel, the screw and barrel temperature were found to have a minor effect on the melting profile. However, these parameters were found to have a significant effect on the predicted pressure profile along the screw channel. It was also observed that the value of the flow rate enforced at the entrance of the screw channel had a large effect on the melting profile and an increase in flow rate resulted in an increase in the solid percentage 
at the same cross-section. When the zero-traction boundary condition was imposed at the entrance of the screw channel, an increase in the screw temperature increased the flow rate in the screw channel, which resulted in an increase in the solid fraction at the same cross-section. However, with the zero-traction boundary condition specified at the entrance, an increase in barrel temperature resulted in a smaller flow rate in the screw channel, and therefore a smaller solid fraction at the same cross-section.

Shear stresses at a sliding interface and the melting flux data for LDPE, LLDPE, ABS, HIPS and PC with different MFRs were presented as a function of temperature and velocity at a pressure of $0.7 \mathrm{MPa}$. The data presented here were consistent with the previous researches in the literature and expanded the data available in the earlier literature. This expanded data set is used for a better understanding of the solids conveying and melting processes in plasticating, single-screw extruders.

An optimization code using the Augmented Lagrangian Method along with the BFGS method was developed to optimize the screw lead and depth in the metering section of a single-screw extruder. This optimization code used a mesh partitioning technique in order to obtain the flow domain. The simulations in this flow domain was performed using the code developed for the polymer melting. The optimization code successfully optimized the screw depth and length such that the average pressure at the entrance of the partitioned barrel was minimum and the temperature at the exit did not exceed the maximum value specified.

\subsection{Recommendations for Future Work}

In Section 3.4, it was mentioned that an unwound channel was used to simulate the two-phase flow in the compression section of a single-screw extruder. With the memory improvement in the computational resources, the assumption of the unwound channel 
can be relaxed and the simulations can be performed for the compression and metering sections of a single-screw extruder using the actual geometry of the single-screw extruder channel, namely the three dimensional helix channel. By using a three dimensional helix channel, not only the curvature effect of the screw channel but also the leakage flow over the flight clearance can be taken into account in the simulation of the melting and flow of polymers in a single-screw extruder. In an unwound channel, the effect of curvature and leakage flow on melting and flow of polymers could not be observed.

It was discussed in Section 2.2.3 that as the melt flow rate (MFR) increases, the average melt film thickness should decrease. In order to support this statement and to investigate the relation between the MFR and the melting flux, numerical simulations are needed.

As discussed in Chapter 4, only two screw geometry parameters were optimized in this work. In the optimization scheme that is described in Chapter 4, in order to calculate the derivative of the objective function with respect to design parameters, $n$ function evaluations are needed where $n$ is the number of design parameters. Thus, an increase in the number of design parameters results in a linear increase in the number of function evaluations. Since the computation of the function evaluations was expensive and occupied the most of the overall computation time, as the number of design parameters increased, the completion time for the optimization code became unfeasible. Instead, adjoint sensitivity analysis method $[98,99]$ may be used to calculate the derivative of the objective function with respect to design parameters. Using this method, only one solution to the system of nonlinear equations is needed regardless of the number of design parameters to calculate the derivatives of the objective function. However, the method needs the storage of some matrices in the memory of the computational resource. Since the hardware of the computational resource used in this work was not sufficient for the storage of these matrices, the adjoint sensitivity method could not be implemented to 
the optimization code discussed in Chapter 4. With the memory improvement on the computational resources, the flow domain for the optimization code can also be extended to the melting section of a single-screw extruder. 


\section{References}

[1] A. Altınkaynak, M. Gupta, M. A. Spalding, and S. L. Crabtree, "Investigation of melting in a single-screw extruder," SPE ANTEC Tech. Papers, pp. 809-813, 2006.

[2] A. Altınkaynak, M. Gupta, M. A. Spalding, and S. L. Crabtree, "Shear stress and melting flux for polycarbonate resins," SPE ANTEC Tech. Papers, pp. $1401-1405$, 2008.

[3] A. Altınkaynak, M. Gupta, M. A. Spalding, and S. L. Crabtree, "An experimental study on shear stress characteristics of polymers in plasticating single-screw extruders," Polymer Engineering \& Science, vol. 49, no. 3, pp. 471-477, 2009.

[4] A. Altınkaynak, M. Gupta, M. A. Spalding, and S. L. Crabtree, "Investigation of the effect of the screw geometry on melting in a single-screw extruder," SPE ANTEC Tech. Papers, 2010.

[5] "Size and impact of the plastics industry on the U.S. economy," Report, SPI, 2008.

[6] Z. Tadmor and I. Klien, Engineering Principles of Plasticating Extrusion. New York: Van Nostrand Reinhold, 1970.

[7] C. Rauwendaal, Polymer Extrusion. New York: Hanser Publisher, 1994.

[8] M. A. Spalding, K. S. Hyun, and B. R. Cohen, "Experimental study of the frictional and viscous forces responsible for solids conveying and melting in single-screw plasticating extruders," SPE ANTEC Tech. Papers, pp. 202 - 210, 1997. 
[9] C. I. Chung, "On the scale-up of plasticating extruder screws.," Polymer Engineering \& Science, vol. 24, no. 9, pp. $626-632,1983$.

[10] B. Maddock, "A visual analysis of flow and mixing in extruder screws," SPE ANTEC Tech. Papers, pp. 383-389, 1959.

[11] L. F. Street, "Plastifying extrusion," International Plastics Engineering, vol. 1, pp. 289-296, 1961.

[12] D. I. Marshall, I. Klein, and R. H. Uhl, "Measurement of screw and plastic temperature profiles in extruders," SPE Journal, vol. 20, no. 4, pp. 329-334, 1964.

[13] D. I. Marshall and I. Klein, "Fundamentals of plasticating extrusion. II. Experiments," Polymer Engineering \& Science, vol. 6, no. 3, pp. 191-197, 1966.

[14] Z. Tadmor, I. Duvdevani, and I. Klein, "Melting in plasticating extuders - Theory and experiments," Polymer Engineering \& Science, vol. 7, no. 3, pp. 198-217, 1967.

[15] G. Martin, "Contribution to determination of melt zone length in thread of singlescrew extruder," Kunststofftechnik, vol. 7, pp. 238-246, 1969.

[16] Z. Tadmor and I. Klein, "The effect of design and operating conditions on melting in plasticating extruders," Polymer Engineering \& Science, vol. 9, no. 1, pp. 1-10, 1969.

[17] G. M. Gale, "Dry-blend extrusion of rigid PVC," Plastics and Polymer, vol. 38, pp. 183-191, 1970.

[18] L. Kacir and Z. Tadmor, "Solids conveying in screw extruders. Part III: The delay zone," Polymer Engineering \& Science, vol. 12, no. 5, pp. 387-395, 1972. 
[19] F. Kulas and N. P. Thorshaug, "PVC powder extrusion. melting properties and particle morphology," Journal of Applied Polymer Science, vol. 23, no. 6, pp. 1781-1794, 1979 .

[20] A. P. D. Cox, J. G. Williams, and D. P. Isherwood, "The melting behavior of a low-density polyethylene powder in a screw extruder," Polymer Engineering \& Science, vol. 21, no. 2, pp. 86-92, 1981.

[21] G. Mennig, "Observations made in the extrusion of wall-slipping rigid PVC," Kunststoffe, vol. 71, pp. 359-362, 1981.

[22] G. Menges and K. P. Klenk, "Melting and plasticating of unplasticized PVC powder in the screw extruder," Kunststoffe, vol. 57, pp. 598-603, 1967.

[23] P. Klenk, "Plastifiziermodelle für die verarbeitung benetzender und nichtbenetzender thermoplaste auf einschnecken-extrudern," Rheologica Acta, vol. 7, pp. 75-78, 1968.

[24] J. Dekker, "Improved screw design for the extrusion of polypropylene," Kunststoffe, vol. 66, pp. 5-7, 1976.

[25] G. Campbell, Z. Tang, C. Wang, and M. Bullwinkel, "Some new observations relative to melting in single screw extruders," SPE ANTEC Tech. Papers, pp. 213-217, 2003.

[26] Z. Tadmor, "Fundamentals of plasticating extrusion. I. A theoretical model for melting," Polymer Engineering \& Science, vol. 6, no. 3, pp. 185-190, 1966.

[27] C. I. Chung, "A new theory for single-screw extrusion: Part I," Modern Plastics, vol. 45, pp. 178-198, 1968.

[28] D. R. Hinrichs and L. U. Lilleleht, "A modified melting model for plastifying extruders," Polymer Engineering \& Science, vol. 10, no. 5, pp. 268-278, 1970. 
[29] R. C. Donovan, "Pressure profiles in plasticating extruders," Polymer Engineering \& Science, vol. 11, no. 6, pp. 484-491, 1971.

[30] R. C. Donovan, "A theoretical melting model for plasticating extruders," Polymer Engineering \& Science, vol. 11, no. 3, pp. 247-257, 1971.

[31] J. R. Vermeulen, P. M. Gerson, and W. J. Beek, "The melting of a bed of polymer granules on a hot moving surface," Chemical Engineering Science, vol. 26, no. 9, pp. 1445-1455, 1971.

[32] J. R. Vermeulen, P. G. Scargo, and W. J. Beek, "The melting of a crystalline polymer in a screw extruder," Chemical Engineering Science, vol. 26, no. 9, pp. 1457-1465, 1971.

[33] I. R. Edmondson and R. T. Fenner, "Melting of thermoplastics in single screw extruders," Polymer, vol. 16, no. 1, pp. 49-56, 1975.

[34] J. T. Lindt, "A dynamic melting model for a single-screw extruder," Polymer Engineering \& Science, vol. 16, no. 4, pp. 284-291, 1976.

[35] J. R. A. Pearson, "On the melting of solids near a hot moving interface, with particular reference to beds of granular polymers," International Journal of Heat and Mass Transfer, vol. 19, no. 4, pp. 405-411, 1976.

[36] J. Shapiro, A. L. Halmos, and J. R. A. Pearson, "Melting in single screw extruders," Polymer, vol. 17, no. 10, pp. 905-918, 1976.

[37] A. L. Halmos, J. R. A. Pearson, and R. Trottnow, "Melting in single screw extruders," Polymer, vol. 19, no. 10, pp. 1199-1216, 1978.

[38] D. W. Sundstrom and J. R. Lo, "Softening rates for polystyrene under shear conditions," Polymer Engineering \& Science, vol. 18, no. 5, pp. 422-426, 1978. 
[39] E. M. Mount and C. I. Chung, "Melting behavior of solid polymers on a metal surface at processing conditions," Polymer Engineering \& Science, vol. 18, no. 9, pp. 711-720, 1978.

[40] J. T. Lindt, "Pressure development in the melting zone of a single-screw extruder," Polymer Engineering \& Science, vol. 21, no. 17, pp. 1162-1166, 1981.

[41] E. Agur and J. Vlachopoulos, "Numerical simulation of a single-screw plasticating extruder," Polymer Engineering \& Science, vol. 22, no. 17, pp. 1084 - 1094, 1982.

[42] E. M. Mount, J. G. Watson, and C. I. Chung, "Analytical melting model for extrusion: Melting rate of fully compacted solid polymers," Polymer Engineering \& Science, vol. 22, no. 12, pp. 729-737, 1982.

[43] I. Bruker and G. S. Balch, "Melting mechanism in single screw extrusion," Polymer Engineering \& Science, vol. 29, no. 4, pp. 258-267, 1989.

[44] H. Potente, "An analytical model of partial and thorough melting in single-screw extruders," International Polymer Processing, vol. 6, pp. 297-303, 1991.

[45] D. W. Sundstrom and C.-C. Young, "Melting rates of crystalline polymers under shear conditions," Polymer Engineering \& Science, vol. 12, no. 1, pp. 59-63, 1972.

[46] C. Chung, W. Hennessey, and M. Tusim, "Frictional behavior of solid polymers on a metal surface at processing conditions," Polymer Engineering and Science, vol. 17, no. 1 , pp. $9-20,1977$.

[47] D. McClelland and C. Chung, "Shear stress at polymer/metal interface during melting in extrusion," Polymer Engineering and Science, vol. 23, no. 2, pp. 100 - 104, 1983. 
[48] K. Chung and C. Chung, "Analytical melting model for extrusion: Stress of fully compacted solid polymers," Polymer Engineering and Science, vol. 23, no. 4, pp. 191 - 196, 1983.

[49] B. Hong, H. Kim, and C. Chung, "Dependence of melting behavior on melt index," SPE ANTEC Tech. Papers, pp. 272-276, 2001.

[50] T. A. Hogan, M. Spalding, K. Cho, and C. Chung, "The melting characteristics of polycarbonate resins," SPE ANTEC Tech. Papers, pp. 384-388, 2002.

[51] A. P. D. Cox and R. T. Fenner, "Melting performance in the single screw extrusion of thermoplastics," Polymer Engineering \& Science, vol. 20, no. 8, pp. 562-571, 1980.

[52] J. T. Lindt and B. Elbirli, "Effect of the cross-channel flow on the melting performance of a single-screw extruder," Polymer Engineering \& Science, vol. 25, no. 7, pp. 412-418, 1985.

[53] E. E. Stangland, J. Dooley, M. Spalding, E. Kim, and K. Hyun, "Fundamental characterization of polypropylene extrusion," SPE ANTEC Tech. Papers, pp. 302-306, 2002.

[54] E. M. Mount III, "Impact of rheological properties on melting rate calculations," SPE ANTEC Tech. Papers, vol. 1, pp. 328 - 334, 2005.

[55] S. Syrjala, "A new approach for the simulation of melting in extruders," International Communications in Heat and Mass Transfer, vol. 27, no. 5, pp. 623-634, 2000.

[56] S. Syrjala, "On the analysis of fluid flow and heat transfer in the melt conveying section of a single-screw extruder," Numerical Heat Transfer: Applications, vol. 35, pp. 25-47, 1999. 
[57] M. Viriyayuthakorn and B. Kassahun, "Three dimensional model for plasticating extrusion screw design," SPE ANTEC Tech. Papers, pp. 81-84, 1984.

[58] K. J. Bathe, Finite Element Procedure. Englewood Cliffs, New Jersey: Prentice Hall, 1996.

[59] A. Mizukami and T. J. R. Hughes, "A Petrov-Galerkin finite element method for convection-dominated flows: An accurate upwinding technique for satisfying the maximum principle," Computer Methods in Applied Mechanics and Engineering, vol. 50, no. 2, pp. 181-193, 1985.

[60] T. J. R. Hughes and M. Mallet, "A new finite element formulation for computational fluid dynamics: III. The generalized streamline operator for multidimensional advective-diffusive systems," Computer Methods in Applied Mechanics and Engineering, vol. 58, no. 3, pp. 305-328, 1986.

[61] T. J. R. Hughes and M. Mallet, “A new finite element formulation for computational fluid dynamics: IV. A discontinuity-capturing operator for multidimensional advective-diffusive systems," Computer Methods in Applied Mechanics and Engineering, vol. 58, no. 3, pp. 329-336, 1986.

[62] T. J. R. Hughes, M. Mallet, and A. Mizukami, "A new finite element formulation for computational fluid dynamics: II. Beyond SUPG," Computer Methods in Applied Mechanics and Engineering, vol. 54, no. 3, pp. 341-355, 1986.

[63] T. J. R. Hughes, L. P. Franca, and G. M. Hulbert, "A new finite element formulation for computational fluid dynamics: VIII. The Galerkin/least-squares method for advective-diffusive equations," Computer Methods in Applied Mechanics and Engineering, vol. 73, no. 2, pp. 173-189, 1989. 
[64] A. N. Brooks and T. J. R. Hughes, "Streamline upwind/Petrov-Galerkin formulations for convection dominated flows with particular emphasis on the incompressible Navier-Stokes equations," Computer Methods in Applied Mechanics and Engineering, vol. 32, pp. 199-259, 1990.

[65] I. Harari and T. J. R. Hughes, "Stabilized finite element methods for steady advection-diffusion with production," Computer Methods in Applied Mechanics and Engineering, vol. 115, pp. 165-191, 1994.

[66] M. A. Spalding, D. E. Kirkpatrick, and K. S. Hyun, "Coefficients of dynamic friction for low density polyethylene," Polymer Engineering and Science, vol. 33, no. 7, pp. $423-430,1993$.

[67] M. A. Spalding and K. S. Hyun, "Coefficients of dynamic friction as a function of temperature, pressure, and velocity for several polyethylene resins," Polymer Engineering \& Science, vol. 35, no. 7, pp. 557-563, 1995.

[68] K. S. Hyun, M. A. Spalding, and C. E. Hinton, "Theoretical and experimental analysis of solids conveying in single-screw extruders," SPE ANTEC Tech. Papers, vol. 1, pp. $199-206,1996$.

[69] M. Spalding, J. Powers, P. Wagner, and K. Hyun, "Flow surging in single-screw plasticating extruders," SPE ANTEC Tech. Papers, pp. 254-258, 2000.

[70] M. Spalding, K. Hyun, and J. Vlachopoulos, The SPE Guide on Extrusion Technology and Troubleshooting, ch. Resin Processing Properties. SPE, 2001.

[71] K. S. Hyun and M. A. Spalding, "A new model for solids conveying in single-screw plasticating extruders," SPE ANTEC Tech. Papers, vol. 1, pp. $211-218,1997$. 
[72] J. R. Powers, M. A. Spalding, and K. S. Hyun, "Extruding HIPS on ABS screws," SPE ANTEC Tech. Papers, pp. 151 - 155, 1994.

[73] K. S. Hyun and M. A. Spalding, "Use of process data obtained from a data acquisition system for optimizing and debugging extrusion processes," Advances in Polymer Technology, vol. 15, no. 1, pp. $29-40,1996$.

[74] T. Butler, Film Extrusion Manual: Process, Materials, Properties, ch. Linear-Low Density Polyethylene. Atlanta, GA: Tappi Press, 2nd ed., 2005.

[75] I. Klein, "What really goes on in the plasticating extruder.," Modern Plastics, vol. 49, no. $11,1972$.

[76] J. N. Reddy and D. K. Gartling, The finite element method in heat transfer and fluid dynamics. CRC Press, 2nd ed., 2000.

[77] S. F. Ashby, T. A. Manteuffel, and P. E. Saylor, "A taxonomy for conjugate gradient methods," SIAM J. Numer. Anal., vol. 27, no. 6, pp. 1542-1568, 1990.

[78] R. Han, Three-dimensional Simulation of Reactive Polymeric Flow during Microchip Encapsulation. PhD thesis, Ph.D. Thesis, Michigan Technological University, 2001.

[79] M. M. Cross, "Relation between viscoelasticity and shear-thinning behavior in liquids," Rheologica Acta, vol. 18, pp. 609-614, 1979.

[80] H. H. Chiang, C. A. Hieber, and K. K. Wang, "A unified simulation of the filling and postfilling stages in injection molding. Part I: Formulation," Polymer Engineering \& Science, vol. 31, no. 2, pp. 116-124, 1991.

[81] PolyXtrue software, Plastic Flow, LLC, 1206 Birch Street, Houghton, MI 49931. 
[82] E. Pichelin and T. Coupez, "Finite element solution of the 3D mold filling problem for viscous incompressible fluid," Computer Methods in Applied Mechanics and Engineering, vol. 163, no. 1-4, pp. 359 - 371, 1998.

[83] C. Catlett and et al., TeraGrid: Analysis of Organization, System Architecture, and Middleware Enabling New Types of Applications. Advances in Parallel Computing, Amsterdam: HPC and Grids in Action, Ed. Lucio Grandinetti, IOS Press 'Advances in Parallel Computing' series, 2007.

[84] N. Bell and M. Garland, "Implementing sparse matrix-vector multiplication on throughput-oriented processors," in SC '09: Proceedings of the Conference on High Performance Computing Networking, Storage and Analysis, (New York, NY, USA), pp. 1-11, ACM, 2009.

[85] S. Syrjala, "Numerical simulation of nonisothermal flow of polymer melt in a single-screw extruder: A validation study," Numerical Heat Transfer, Part A: Applications: An International Journal of Computation and Methodology, vol. 37, no. 8 , pp. $897-915,2000$.

[86] S. J. Derezinski, "Heat transfer in extruder screws," SPE ANTEC Tech. Papers, pp. 83-93, 2000.

[87] A. G. Cunha, Modelling and Optimisation of Single Screw Extrusion. PhD thesis, Ph.D. Thesis, University of Minho, 2000.

[88] A. J. Bur, S. C. Roth, M. A. Spalding, D. W. Baugh, K. A. Koppi, and W. C. Buzanowski, "Temperature gradients in the channels of a single-screw extruder," Polymer Engineering and Science, vol. 44, no. 11, pp. 2148-2157, 2004. 
[89] F. Zhu and L. Chen, "Studies on the theory of single screw plasticating extrusion. Part I: A new experimental method for extrusion," Polymer Engineering \& Science, vol. 31, no. 15, pp. 1113-1116, 1991.

[90] R. V. Chiruvella, Y. Jaluria, V. Sernas, and M. Esseghir, "Extrusion of non-newtonian fluids in a single-screw extruder with pressure back flow," Polymer Engineering \& Science, vol. 36, no. 3, pp. 358-367, 1996.

[91] V. Sernas, L. Xiao, P. Lin, and Y. Jaluria, "Axial conduction in a convectively heated/cooled extruder barrel," SPE ANTEC Tech. Papers, pp. 422-426, 1996.

[92] M. Gupta, "Mesh partitioning technique for three-dimensional simulation of coextrusion," SPE ANTEC Tech. Papers, pp. 217-222, 2008.

[93] M. Gupta, "Three-dimensional simulation of profile coextrusion using mesh partitioning technique," SPE Coextrusion TOPCON Tech. Papers, pp. 108 - 113, 2008.

[94] M. Gupta, V. Rohatgi, and R. Kuehn, "Estimation of temperature increase in a zsk-90 co-rotating twin-screw extruder using mesh partitioning technique," SPE ANTEC Tech. Papers, vol. 4, pp. $2350-2355,2009$.

[95] J. Nocedal and S. J. Wright, Numerical Optimization. New York: Springer-Verlag, 1st ed., 1999.

[96] S. S. Rao, Engineering Optimization: Thoery and Practice. Hoboken, New Jersey: John Wiley \& Sons, 4th ed., 2009.

[97] J. Nocedal and S. J. Wright, Numerical Optimization. New York: Springer-Verlag, 2nd ed., 2006. 
[98] D. A. Tortorelli and P. Michaleris, "Design sensitivity analysis: Overview and review," Inverse Problems in Engineering, vol. 1, no. 1, pp. 71-105, 1994.

[99] Y. Sun, Optimization of Polymer Geometry for Polymer Extrusion. PhD thesis, Ph.D. Thesis, Michigan Technological University, 2006. 


\section{Appendix A}

\section{Copyright Agreement}

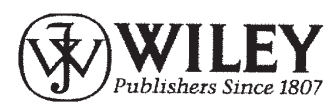

Date:

$09 / 19 / 2008$

\section{River Street Hoboken, NJ 07030 \\ COPYRIGHT TRANSFER AGREEMENT $\quad 201.748 .6000$ \\ $\begin{array}{ll}\text { COPYRIGHT TRANSFER AGREEMENT } & 201.748 .6000 \\ & \text { FAX 201.748.6052 }\end{array}$}

To:

Production/Contribution

ID\#

Publisher/Editorial office use only

Re: Manuscript entitled AN EXPERIMENTAL STUOY ON SHEAR STRESS CHARACTIZRISTLS OF POLYMERS IN PLATICATING SINGLE SCREW EXTEUDRES (the "Contribution") for publication in POLYMER ENGWNERINGQ SCIENCE "Journal") published by Wiley Periodicals, Inc. ("Wiley").

Dear Contributor(s):

Thank you for submitting your Contribution for publication. In order to expedite the editing and publishing process and enable Wiley to disseminate your work to the fullest extent, we need to have this Copyright Transfer Agreement signed and returned to us as soon as possible. If the Contribution is not accepted for publication this Agreement shall be null and void.

\section{A. COPYRIGHT}

1. The Contributor assigns to Wiley, during the full term of copyright and any extensions or renewals of that term, all copyright in and to the Contribution, including but not limited to the right to publish, republish, transmit, sell, distribute and otherwise use the Contribution and the material contained therein in electronic and print editions of the Journal and in derivative works throughout the world, in all languages and in all media of expression now known or later developed, and to license or permit others to do so.

2. Reproduction, posting, transmission or other distribution or use of the Contribution or any material contained therein, in any medium as permitted hereunder, requires a citation to the Journal and an appropriate credit to Wiley as Publisher, suitable in form and content as follows: (Title of Article, Author, Journal Title and Volume/Issue Copyright (C) [year] Wiley Periodicals, Inc. or copyright owner as specified in the Journal.)

B. RETAINED RIGHTS

Notwithstanding the above, the Contributor or, if applicable, the Contributor's Employer, retains all proprietary rights other than copyright, such as patent rights, in any process, procedure or article of manufacture described in the Contribution, and the right to make oral presentations of material from the Contribution.

\section{OTHER RIGHTS OF CONTRIBUTOR}

Wiley grants back to the Contributor the following:

1. The right to share with colleagues print or electronic "preprints" of the unpublished Contribution, in form and content as accepted by Wiley for publication in the Journal. Such preprints may be posted as electronic files on the Contributor's own website for personal or professional use, or on the Contributor's internal university or corporate networks/intranet, or secure external website at the Contributor's institution, but not for commercial sale or for any systematic external distribution by a third party (e.g., a listserve or database connected to a public access server). Prior to publication, the Contributor must include the following notice on the preprint: "This is a preprint of an article accepted for publication in [Journal title] (c) copyright (year) (copyright owner as specified in the Journal)". After publication of the Contribution by Wiley, the preprint notice should be amended to read as follows: "This is a preprint of an article published in [include the complete citation information for the final version of the Contribution as published in the print edition of the Journal]", and should provide an electronic link to the Journal's WWW site, located at the following Wiley URL: http://www.interscience.Wiley.com/. The Contributor agrees not to update the preprint or replace it with the published version of the Contribution.

2. The right, without charge, to photocopy or to transmit online or to download, print out and distribute to a colleague a copy of the published Contribution in whole or in part, for the colleague's personal or professional use, for the 
advancement of scholarly or scientific research or study, or for corporate informational purposes in accordance with Paragraph D.2 below.

3. The right to republish, without charge, in print format, all or part of the material from the published Contribution in a book written or edited by the Contributor.

4. The right to use selected figures and tables, and selected text (up to 250 words, exclusive of the abstract) from the Contribution, for the Contributor's own teaching purposes, or for incorporation within another work by the Contributor that is made part of an edited work published (in print or electronic format) by a third party, or for presentation in electronic format on an internal computer network or external website of the Contributor or the Contributor's employer.

5. The right to include the Contribution in a compilation for classroom use (course packs) to be distributed to students at the Contributor's institution free of charge or to be stored in electronic format in datarooms for access by students at the Contributor's institution as part of their course work (sometimes called "electronic reserve rooms") and for inhouse training programs at the Contributor's employer.

\section{CONTRIBUTIONS OWNED BY EMPLOYER}

1. If the Contribution was written by the Contributor in the course of the Contributor's employment (as a "work-madefor-hire" in the course of employment), the Contribution is owned by the company/employer which must sign this Agreement (in addition to the Contributor's signature), in the space provided below. In such case, the company/employer hereby assigns to Wiley, during the full term of copyright, all copyright in and to the Contribution for the full term of copyright throughout the world as specified in paragraph A above.

2. In addition to the rights specified as retained in paragraph B above and the rights granted back to the Contributor pursuant to paragraph $\mathrm{C}$ above, Wiley hereby grants back, without charge, to such company/employer, its subsidiaries and divisions, the right to make copies of and distribute the published Contribution internally in print format or electronically on the Company's internal network. Upon payment of Wiley's reprint fee, the institution may distribute (but not resell) print copies of the published Contribution externally. Although copies so made shall not be available for individual re-sale, they may be included by the company/employer as part of an information package included with software or other products offered for sale or license. Posting of the published Contribution by the institution on a public access website may only be done with Wiley's written permission, and payment of any
applicable fee(s).

\section{E. GOVERNMENT CONTRACTS}

In the case of a Contribution prepared under U.S. Government contract or grant, the U.S. Government may reproduce, without charge, all or portions of the Contribution and may authorize others to do so, for official U.S. Government purposes only, if the U.S. Government contract or grant so requires. (U.S. Government Employees: see note at end.)

\section{F. COPYRIGHT NOTICE}

The Contributor and the company/employer agree that any and all copies of the Contribution or any part thereof distributed or posted by them in print or electronic format as permitted herein will include the notice of copyright as stipulated in the Journal and a full citation to the Journal as published by Wiley.

\section{G. CONTRIBUTOR'S REPRESENTATIONS}

The Contributor represents that the Contribution is the Contributor's original work. If the Contribution was prepared jointly, the Contributor agrees to inform the co-Contributors of the terms of this Agreement and to obtain their signature to this Agreement or their written permission to sign on their behalf. The Contribution is submitted only to this Journal and has not been published before, except for "preprints" as permitted above. (If excerpts from copyrighted works owned by third parties are included, the Contributor will obtain written permission from the copyright owners for all uses as set forth in Wiley's permissions form or in the Journal's Instructions for Contributors, and show credit to the sources in the Contribution.) The Contributor also warrants that the Contribution contains no libelous or unlawful statements, does not infringe upon the rights (including without limitation the copyright, patent or trademark rights) or the privacy of others, or contain material or instructions that might cause harm or injury. 
CHECK ONE:

$[, X]$ Contributor-owned work

$\begin{array}{ll}\text { Contributor's signature } & \frac{09 / 19 / 2008}{\text { Date }} \\ \frac{M R \text {. ATAKAN ALTNKAYNAK }}{\text { Type or print name and title }} & \frac{09 / 19 / 08}{\text { Date }} \\ \frac{\text { Mo-contributor's signature }}{\text { MAHESH GUPTA }} & \text { PAefessrs }\end{array}$

ATTACHED ADDITIONAL SIGNATURE PAGE AS NECESSARY

[__ Company/Institution-owned work (made-for-hire in the

Company or Institution (Employer-for-Hire)

Date

course of employment)

Authorized signature of Employer

Date

U.S. Government work

Note to U.S. Government Employees

A contribution prepared by a U.S. federal government employee as part of the employee's official duties, or which is an official U.S. Government publication is called a "U.S. Government work," and is in the public domain in the United States. In such case, the employee may cross out Paragraph A.1 but must sign and return this Agreement. If the Contribution was not prepared as part of the employee's duties or is not an official U.S. Government publication, it is not a U.S. Government work.

[ ] U.K. Government work (Crown Copyright)

Note to U.K. Government Employees

The rights in a Contribution prepared by an employee of a U.K. government department, agency or other Crown body as part of his/her official duties, or which is an official government publication, belong to the Crown. In such case, Wiley will forward the relevant form to the Employee for signature. 